DOE/ER/13335--1

DE93 000404

\title{
CRYOTRIBOLOGY: DEVELOPMENT OF CRYOTRIBOLOGICAL THEORIES AND APPLICATION TO CRYOGENIC DEVICES
}

\author{
Interim Report \\ for Period June 15, 1985 - August 31, 1992 \\ Y. Iwasa, E. Rabinowicz ${ }^{\dagger}$, and P. Michael \\ Francis Bitter National Laboratory and \\ †Department of Mechanical Engineering \\ Massachusetts Institute of Technology \\ Cambridge, MA 02139
}

September 15, 1992

Prepared for

US Department of Energy

Office of Basic Energy Sciences

Germantown, MD 20585

Agreement No. DE-FG02-85ER13335.A006

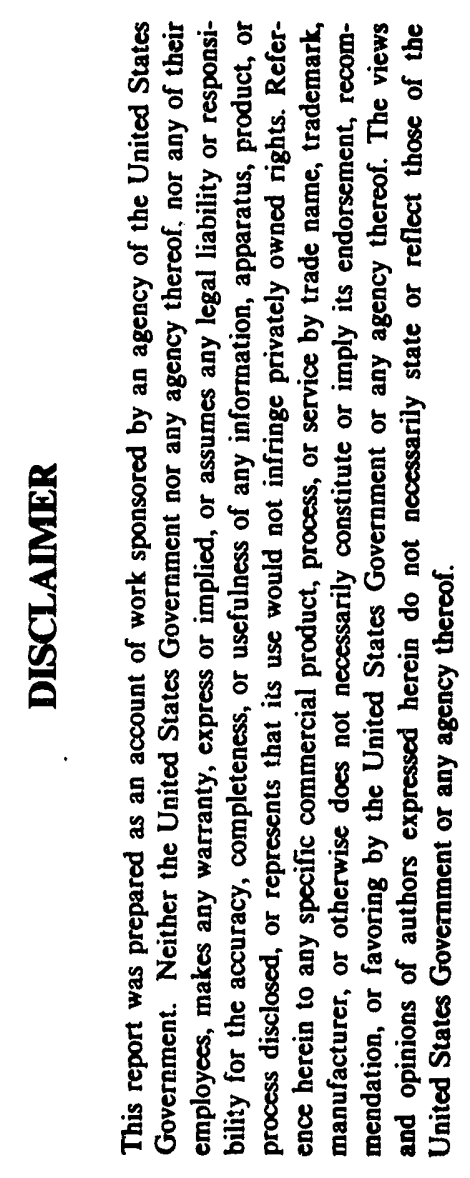




\section{NOTICE}

This report was prepared as an account of work sponsored by the United States Government. Neither the United States nor the Department of Energy, nor any of their employees, nor any of their contractors, subcontractors, or their employees, makes any warranty, express or implied, or assumes any legal liability or responsibility for the accuracy, completeness, or usefulness of any information, apparatus, product or process disclosed or represents that its use would not infringe privately-owed rights. 


\begin{abstract}
High-performance superconducting solenoids are susceptible to premature quenches, or superconducting to normal state transitions, due to abrupt conductor movements within the winding. Both theoretical prediction and experimental evidence indicate that abrupt motions involving $5 \sim 10 \mu \mathrm{m}$ conductor displacements dissipate sufficient energy to trigger a quench. The sliding and mechanical behaviors of materials at cryogenic temperatures have been experimentally examined. After accounting for changes in the sliding materials' low-temperature strength properties, we have found that the adhesion theory of friction and wear remains applicable at cryogenic temperatures.

The adhesion friction theory suggests two methods for controlling unsteady sliding motions. The first involves the selection of sliding materials whose friction coefficients increase with increasing sliding speed. A number of material pairs have been examined, both theoretically and experimentally, for positive friction-velocity characteristics. We conclude that this materials-based approach to frictional stabilization does not seem to be a viable option at $4.2 \mathrm{~K}$, the normal operating temperature for contemporary superconducting magnets.

The second alternative is to preprogram the force conditions within 'high-risk' regions of the winding to regulate the occurrence of unsteady sliding motions. Structural models are proposed to account for unsteady conductor motions on a variety of dimensional scales. The models are subsequently used to design a small superconducting solenoid. The performance of this solenoid suggests that force-based motion control is a potentially viable design approach for achieving successful dry-wound magnets.
\end{abstract}




\section{ACKNOW/LEDGEMENTS}

The authors gratefully acknowledge the contribution of Professor Ioannis Yannas of MIT's Mechanical Engineering Department who provided valuable insights into low-temperature polymeric-material mechanical behavior.

Thanks are also due to Dr. Emanuel Bobrov of the Francis Bitter National Laboratory who assisted in the design of the small, superconducting test coil presented in Chapter VII.

This work was supported by funds from the United States Department of Energy, Office of Basic Energy Sciences. 


\section{Table of Contents}

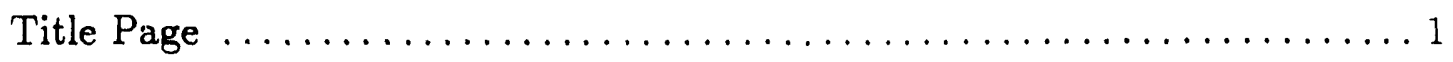

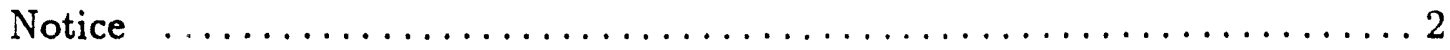

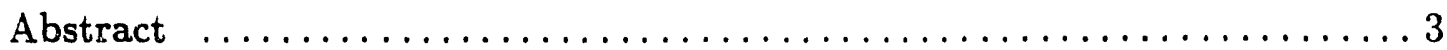

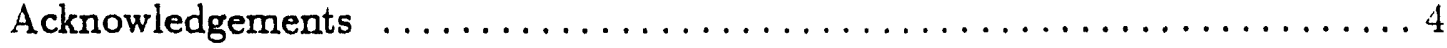

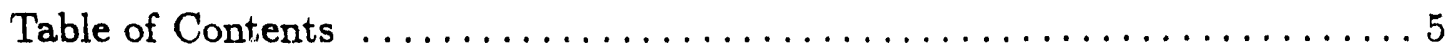

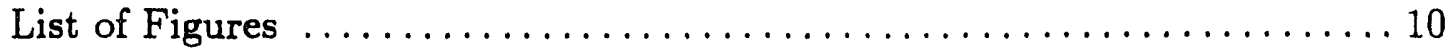

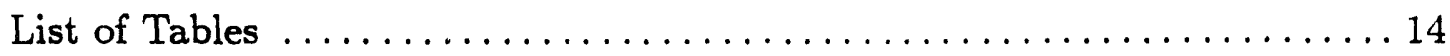

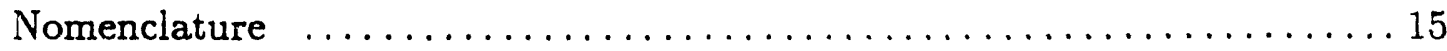

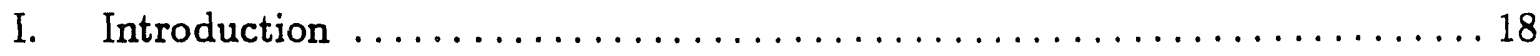

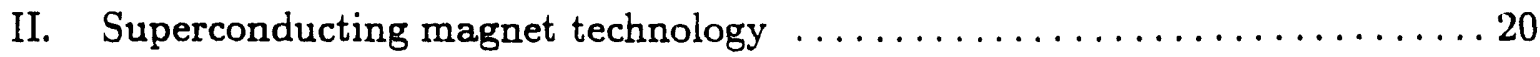

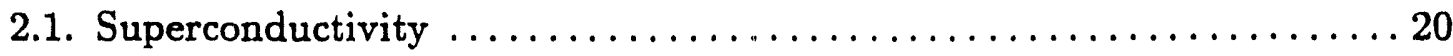

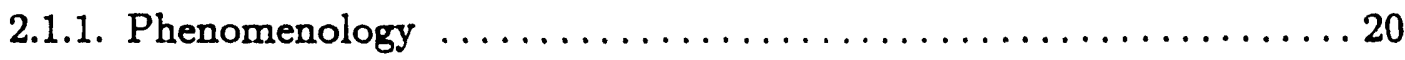

2.1.2. Commercial, composite superconducting wires $\ldots \ldots \ldots \ldots \ldots 21$

2.2. Superconducting magnet applications $\ldots \ldots \ldots \ldots \ldots \ldots \ldots \ldots \ldots$

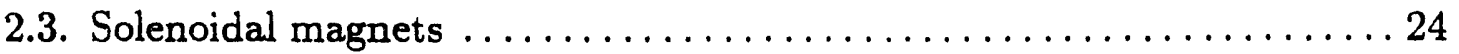

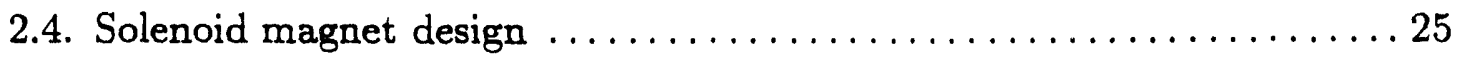

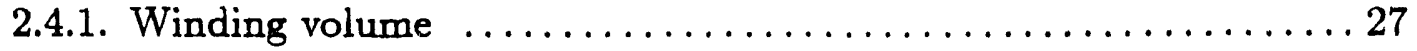

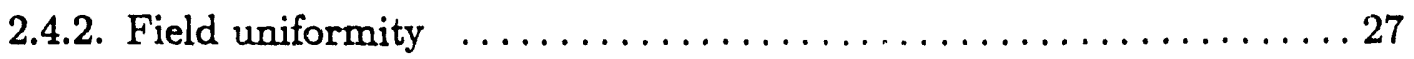

2.4.3. Superconductor current density $\ldots \ldots \ldots \ldots \ldots \ldots \ldots \ldots \ldots \ldots \ldots \ldots \ldots \ldots$

2.4.4. Superconductor volume fraction $\ldots \ldots \ldots \ldots \ldots \ldots \ldots \ldots \ldots \ldots$

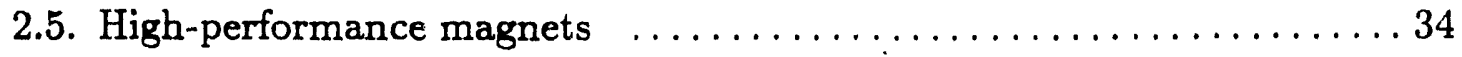

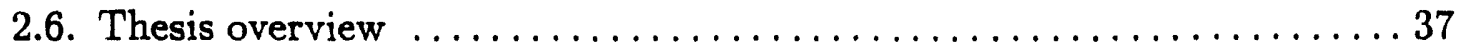

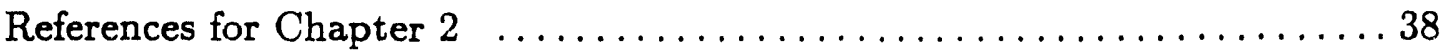

III. Mechanical-disturbance-indured quenches $\ldots \ldots \ldots \ldots \ldots \ldots \ldots \ldots$

3.1. Thermal stability of high-performance magnets $\ldots \ldots \ldots \ldots \ldots \ldots . \ldots 39$ 
3.1.1. Energy balance for unit winding volume $\ldots \ldots \ldots \ldots \ldots \ldots \ldots$

3.1.2. Continuous disturbances $\ldots \ldots \ldots \ldots \ldots \ldots \ldots \ldots \ldots \ldots \ldots$

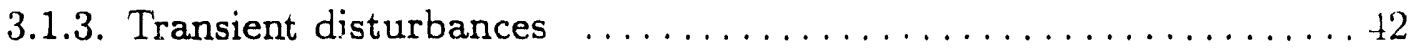

3.1.3.1. Distributed disturbances $\ldots \ldots \ldots \ldots \ldots \ldots \ldots \ldots \ldots \ldots$

3.1.3.2. Point disturbances - minimum propagating zones $\ldots \ldots \ldots .43$

3.2. Characterization of transient point disturbances $\ldots \ldots \ldots \ldots \ldots \ldots 46$

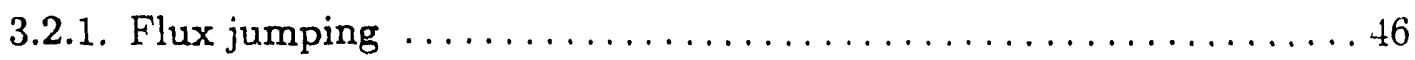

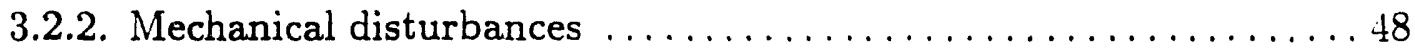

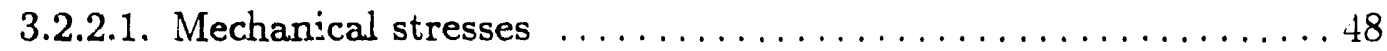

3.2.2.2. Disturbance characterization $\ldots \ldots \ldots \ldots \ldots \ldots \ldots \ldots$

3.3. Mechanical disturbance detection $\ldots \ldots \ldots \ldots \ldots \ldots \ldots \ldots \ldots \ldots \ldots \ldots \ldots \ldots \ldots$

3.3.1. Strain gauges $\ldots \ldots \ldots \ldots \ldots \ldots \ldots \ldots \ldots \ldots \ldots \ldots \ldots \ldots \ldots \ldots \ldots \ldots \ldots \ldots$

3.3.2. Acoustic emissior s monitoring $\ldots \ldots \ldots \ldots \ldots \ldots \ldots \ldots \ldots$

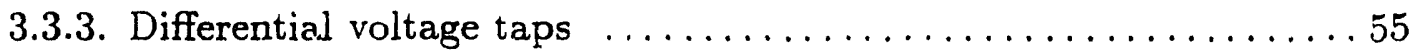

3.3.4. Disturbance monitoring $\ldots \ldots \ldots \ldots \ldots \ldots \ldots \ldots \ldots \ldots \ldots \ldots \ldots \ldots \ldots \ldots \ldots \ldots$

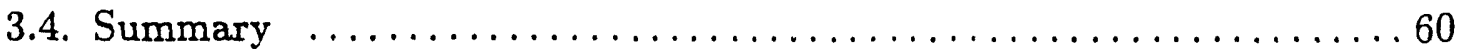

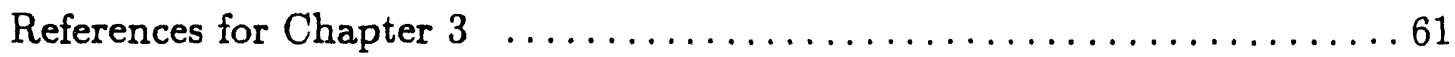

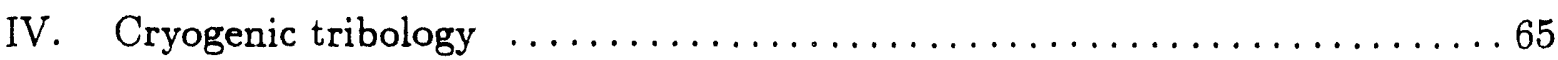

4.1. Asperity junction model for adhesive friction and wear $\ldots \ldots \ldots \ldots 65$

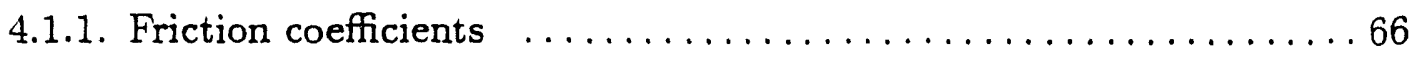

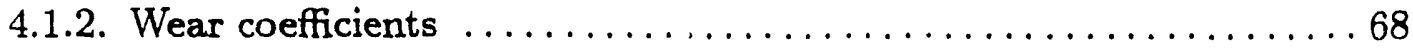

4.1.3. Relation between wear and friction $\ldots \ldots \ldots \ldots \ldots \ldots 6$

4.2. Experimental verification of adhesive mechanism at $4.2 \mathrm{~K} \ldots \ldots \ldots 6$

4.2.1. Description of test materials $\ldots \ldots \ldots \ldots \ldots \ldots \ldots \ldots \ldots \ldots \ldots \ldots \ldots$

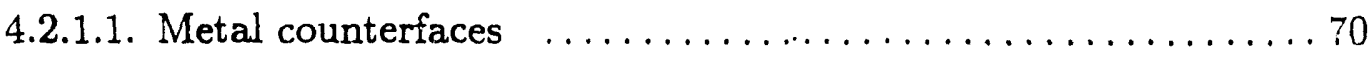

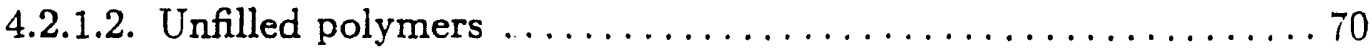

4.2.1.3. Fabric- and particle-filled polymers $\ldots \ldots \ldots \ldots \ldots \ldots \ldots$

4.2.2. Mechanical characterization of test materials $\ldots \ldots \ldots \ldots \ldots \ldots 72$ 
4.2.3. Pin-on-disk experiments $\ldots \ldots \ldots \ldots \ldots \ldots \ldots \ldots \ldots \ldots 77$

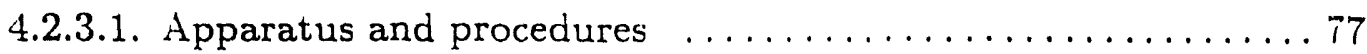

4.2.3.2. Sample preparation $\ldots \ldots \ldots \ldots \ldots \ldots \ldots \ldots \ldots \ldots \ldots \ldots \ldots \ldots \ldots \ldots$

4.2.4. Experimental results $\ldots \ldots \ldots \ldots \ldots \ldots \ldots \ldots \ldots \ldots \ldots \ldots \ldots \ldots \ldots \ldots$

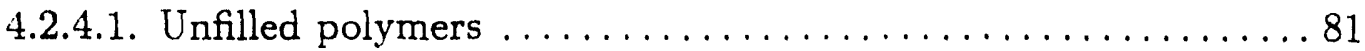

4.2.4.2. Fabric- and particle-filled polymers $\ldots \ldots \ldots \ldots \ldots \ldots \ldots$

4.3. Theoretical summary of experimental data $\ldots \ldots \ldots \ldots \ldots \ldots$

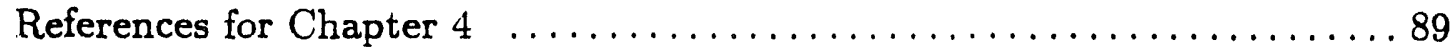

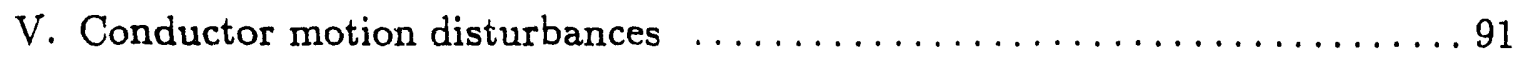

5.1. Frictional characteristics of cryostable magnets $\ldots \ldots \ldots \ldots \ldots \ldots 1$

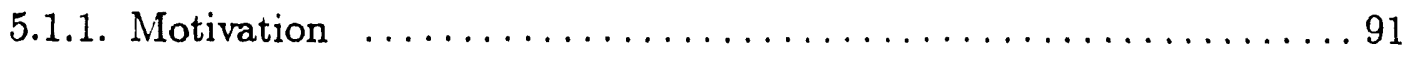

5.1.2. Superconductor-insulator friction $\ldots \ldots \ldots \ldots \ldots \ldots 2$

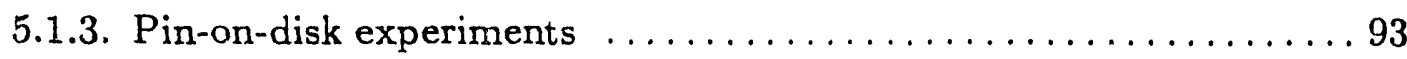

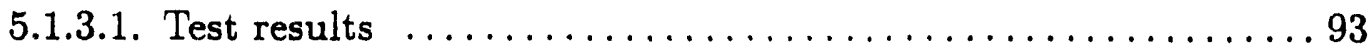

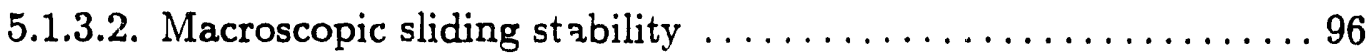

5.1.4. Linear friction experiments $\ldots \ldots \ldots \ldots \ldots \ldots \ldots \ldots \ldots \ldots$

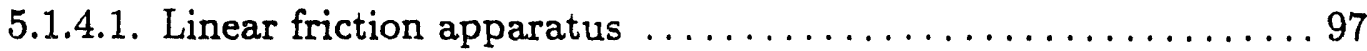

5.1.4.2. Friction force-displacement diagrams $\ldots \ldots \ldots \ldots \ldots \ldots . \ldots 99$

5.1.4.3. Acoustic emission during sliding $\ldots \ldots \ldots \ldots \ldots \ldots \ldots$

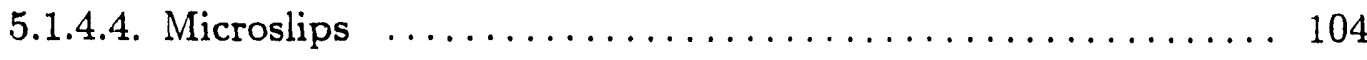

5.2. Spring/electromagnetic-force/friction model ............. 108

5.3. Conductor motions in high-performance solenoids $\ldots \ldots \ldots \ldots \ldots 114$

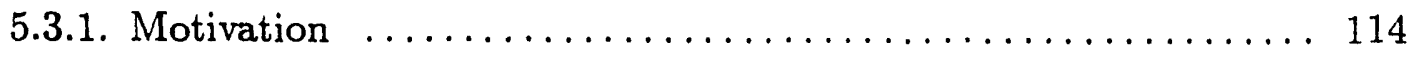

5.3.2. Probable quench initiation locations $\ldots \ldots \ldots \ldots \ldots \ldots \ldots$

5.3.3. Conductor-motion stabilization $\ldots \ldots \ldots \ldots \ldots \ldots \ldots \ldots$

5.3.4. Friction of dry-winding constituents $\ldots \ldots \ldots \ldots \ldots \ldots \ldots \ldots$

5.4. Summary $\ldots \ldots \ldots \ldots \ldots \ldots \ldots \ldots \ldots \ldots \ldots \ldots \ldots \ldots \ldots \ldots \ldots \ldots$

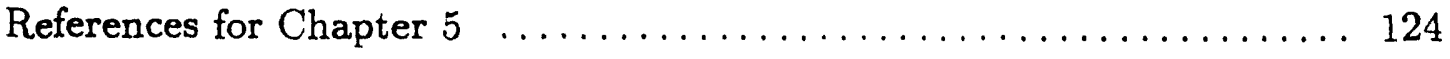


VI. Cryogenic sliding stabilization $\ldots \ldots \ldots \ldots \ldots \ldots \ldots \ldots \ldots \ldots \ldots, 127$

6.1. Stick-slip instabilities ........................... 127

6.1.1. Stick-slip model ................................ 128

6.1.2. Deformation during the stick portion of the stick-slip cycle $\ldots 128$

6.1.3. Velocity-controlled stick-slip $\ldots \ldots \ldots \ldots \ldots \ldots \ldots \ldots \ldots, 131$

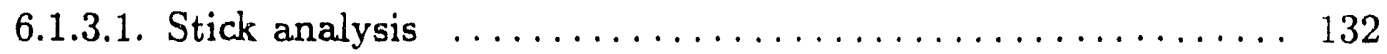

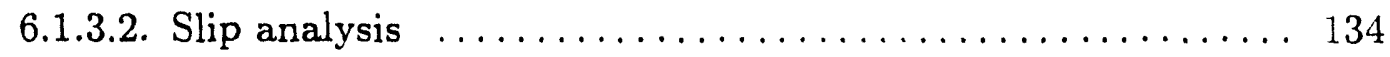

6.1.3.3. Stick-slip prevention $\ldots \ldots \ldots \ldots \ldots \ldots \ldots \ldots \ldots \ldots, 135$

6.2. Viscoelastic sliding stabilization $\ldots \ldots \ldots \ldots \ldots \ldots \ldots \ldots \ldots, 136$

6.2.1. Regions of mechanical equivalence $\ldots \ldots \ldots \ldots \ldots \ldots \ldots \ldots, 137$

6.2.2. Time-temperature superposition in elastomer friction $\ldots \ldots \ldots, 140$

6.2.3. Viscoelastic friction model $\ldots \ldots \ldots \ldots \ldots \ldots \ldots \ldots \ldots, 141$

6.2.4. Friction-temperature behavior $\ldots \ldots \ldots \ldots \ldots \ldots \ldots \ldots, 145$

6.2.5. Low-temperature polymer friction $\ldots \ldots \ldots \ldots \ldots \ldots \ldots \ldots .145$

6.2.6. Screening techniques for low-temperature friction materials $\ldots .149$

6.2.7. Low-temperature sliding initiation $\ldots \ldots \ldots \ldots \ldots \ldots \ldots \ldots 151$

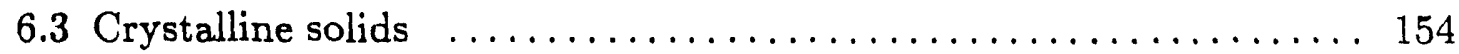

6.3.1. Creep theory of friction $\ldots \ldots \ldots \ldots \ldots \ldots \ldots \ldots \ldots \ldots \ldots \ldots \ldots \ldots$

6.3.1.1. Positive friction-velocity characteristics ............. 154

6.3.1.2. Negative friction-velocity characteristics $\ldots \ldots \ldots \ldots \ldots \ldots$

6.3.2. Time-temperature superposition in crystalline solids $\ldots \ldots \ldots \ldots 156$

6.3.2.1. Thermally activated fatty-acid friction $\ldots \ldots \ldots \ldots \ldots .157$

6.3.2.2. Mechanical relaxations in fatty-acid friction $\ldots \ldots \ldots \ldots .161$

6.3.3. Metallic, friction-temperature effects . ............... 163

6.4. Conductor stiffness modelling $\ldots \ldots \ldots \ldots \ldots \ldots \ldots \ldots \ldots \ldots, 167$

6.4.1. Short conductor segment slips $\ldots \ldots \ldots \ldots \ldots \ldots \ldots \ldots \ldots, 168$

6.4.2. Whole turn conductor stiffness $\ldots \ldots \ldots \ldots \ldots \ldots \ldots \ldots \ldots, 170$

6.4.3. Rigid body coil motions $\ldots \ldots \ldots \ldots \ldots \ldots \ldots \ldots \ldots \ldots . \ldots \ldots$ 


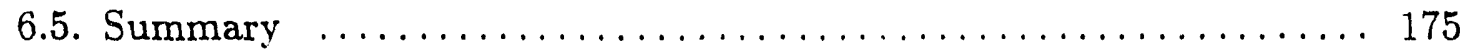

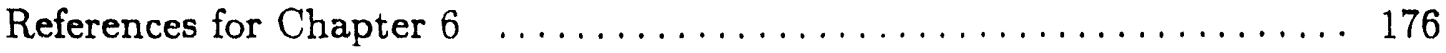

VII. Development of a dry-wound insert coil $\ldots \ldots \ldots \ldots \ldots \ldots \ldots \ldots, 180$

7.1. Structural characterization of dry-wound solenoids ........... 180

7.2. Extension of dry-winding technique to high-field solenoids . ...... 182

7.3. Overview of insert coil design $\ldots \ldots \ldots \ldots \ldots \ldots \ldots \ldots \ldots \ldots \ldots \ldots$

7.3.1. Electromagnetic design $\ldots \ldots \ldots \ldots \ldots \ldots \ldots \ldots \ldots \ldots \ldots$

7.3.2. Mechanical design $\ldots \ldots \ldots \ldots \ldots \ldots \ldots \ldots \ldots \ldots \ldots \ldots, 187$

7.3.2.1. In-plane stresses $\ldots \ldots \ldots \ldots \ldots \ldots \ldots \ldots \ldots \ldots \ldots \ldots . \ldots \ldots 2$

7.3.2.2. Thermal contraction stresses $\ldots \ldots \ldots \ldots \ldots \ldots \ldots \ldots . \ldots 194$

7.3.2.3. Residual winding stresses $\ldots \ldots \ldots \ldots \ldots \ldots \ldots \ldots \ldots \ldots$

7.3.2.4. Electromagnetic stresses $\ldots \ldots \ldots \ldots \ldots \ldots \ldots \ldots \ldots . \ldots 197$

7.4. Application of electromagnetic-spring-friction force model to coil

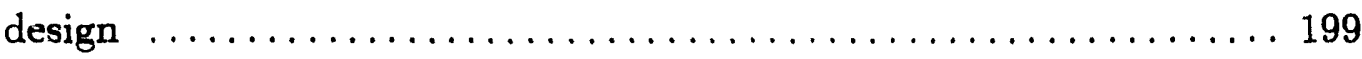

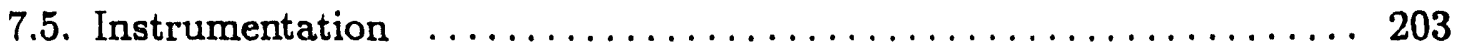

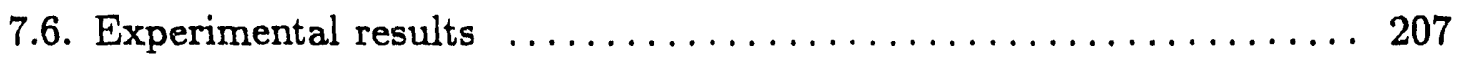

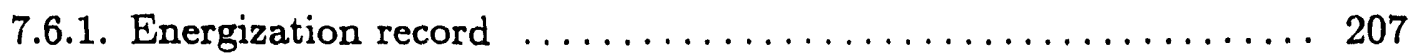

7.6.2. Quench characterization $\ldots \ldots \ldots \ldots \ldots \ldots \ldots \ldots \ldots \ldots . \ldots \ldots$

7.6.3. Disturbance histories $\ldots \ldots \ldots \ldots \ldots \ldots \ldots \ldots \ldots \ldots \ldots, 211$

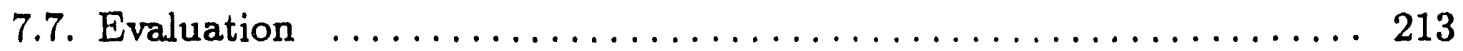

References for Chapter 7 ........................ 217

VIII. Conclusions and recommendations $\ldots \ldots \ldots \ldots \ldots \ldots \ldots \ldots \ldots \ldots 218$

8.1 Friction modification $\ldots \ldots \ldots \ldots \ldots \ldots \ldots \ldots \ldots \ldots \ldots \ldots \ldots \ldots \ldots \ldots \ldots, 218$

8.2. Structural stabilization $\ldots \ldots \ldots \ldots \ldots \ldots \ldots \ldots \ldots \ldots \ldots \ldots$

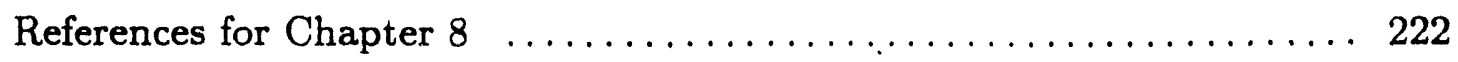

Appendix A. Magnet field maps $\ldots \ldots \ldots \ldots \ldots \ldots \ldots \ldots \ldots \ldots . \ldots \ldots$

Appendix B. Derivation of orthotropic winding properties $\ldots \ldots \ldots \ldots 228$

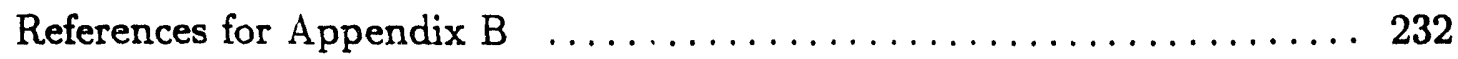




\section{List of Figures}

Figure 2.1: Cross-sectional view of commercial. composite superconducting wires, consisting of fine $\mathrm{NbTi}$ filaments imbedded in a

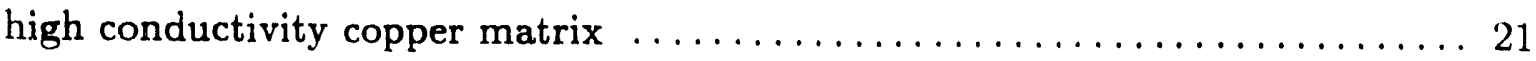

Figure 2.2: Schematic representation of a simple solenoidal winding $\ldots \ldots 26$

Figure 2.3: Parameterized curves of constant $B_{w} / B_{0}$ in a simple

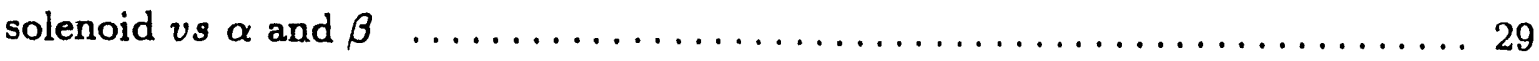

Figure 2.4: Idealized load line and critical superconducting properties

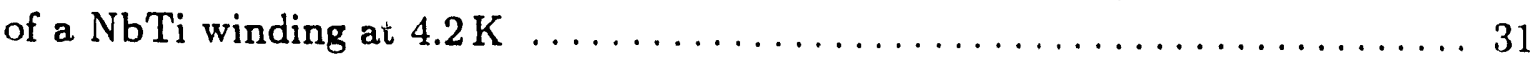

Figure 2.5: Curves of constant $F(\alpha, \beta)$ and $v(\alpha, \beta)$ vs $\alpha$ and $\beta \ldots \ldots 33$

Figure 2.6: Winding models for high-performance magnets constructed from a) circular and b) rectangular cross-sectioned conductors $\ldots \ldots \ldots \ldots . .36$

Figure 3.1: Parameterized curves of quench energy density vs normalized operating current, at various fixed magnetic flux densities, for a 'typical' NbTi winding at $4.2 \mathrm{~K} \ldots \ldots \ldots \ldots \ldots \ldots \ldots \ldots \ldots \ldots \ldots \ldots \ldots$

Figure 3.2: "Minimum" quench energy vs pulse width for an epoxy impregnated superconducting winding $\ldots \ldots \ldots \ldots \ldots \ldots \ldots \ldots \ldots$

Figure 3.3: a) Parameterized contours of constant magnetic induction.

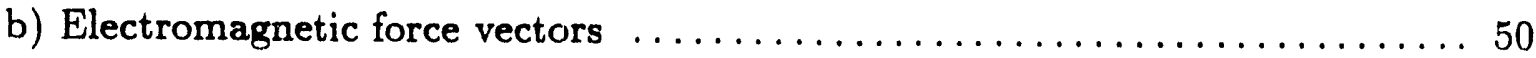

Figure 3.4: Example of the Kaiser effect observed during friction experiment between OFHC copper and G-10 $\ldots \ldots \ldots \ldots \ldots \ldots \ldots \ldots$

Figure 3.5: Simple model to predict the characteristics of the voltage transient produced by conductor motion in background magnetic

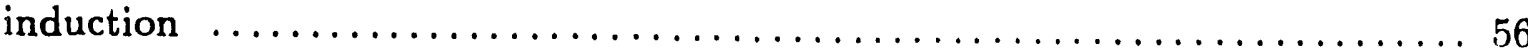

Figure 3.6: AE and voltage traces recorded at initiation of conductor motion induced quench $\ldots \ldots \ldots \ldots \ldots \ldots \ldots \ldots \ldots \ldots \ldots$

Figure 4.1: Indentation hardness test apparatus. consisting of modified Rockwell tester and liquid nitrogen reservoir $\ldots \ldots \ldots \ldots \ldots \ldots \ldots \ldots$ 
Fig,ure 4.2: Schematic cross-section of the pin-on-disk apparatus $\ldots \ldots \ldots 78$

Figure 4.3: Average wear coefficient vs friction coefficient for the

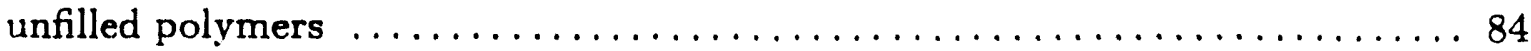

Figure 4.4: Average wear coefficient $v s$ friction coefficient for the fabric- and particle-filled polymers $\ldots \ldots \ldots \ldots \ldots \ldots \ldots \ldots \ldots \ldots$

Figure 5.1: Friction-velocity diagrams $\ldots \ldots \ldots \ldots \ldots \ldots \ldots \ldots \ldots \ldots$

Figure 5.2: Friction-velocity traces from pin-on-disk tests at each of three test temperatures for a) copper/nylon and b) copper/G-10 sliding pairs .... 95

Figure 5.3: Schematic illustration of linear friction apparatus used during transient slip experiments $\ldots \ldots \ldots \ldots \ldots \ldots \ldots \ldots \ldots \ldots$

Figure 5.4: $4.2 \mathrm{~K}$ friction force-displacement diagrams $\ldots \ldots \ldots \ldots \ldots \ldots . \ldots 0$

Figure 5.5: $4.2 \mathrm{~K}$ friction force-displacement diagrams during running-in of a) copper/nylon and b) copper/G-10 pairs ............ 102

Figure 5.6: Acoustic count rate, tangential force, and total displacement vs time for a copper/G-10 transient slip experiment $\ldots \ldots \ldots \ldots \ldots \ldots \ldots 105$

Figure 5.7: Correspondence between AE burst and magnetic

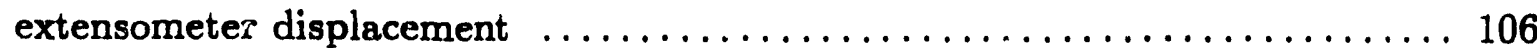

Figure 5.8: Schematic illustration of moving conductor segment in

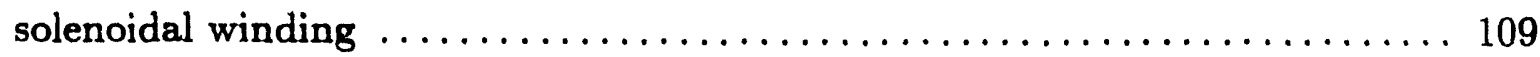

Figure 5.8: Spring-electromagnetic-friction force model used to interpret reversibility of conductor motion

Figure 5.10: Results of conductor motion experiments on single layer solenoidal winding showing cumulative AE vs current $\ldots \ldots \ldots \ldots \ldots \ldots 113$

Figure 5.11: Results of quench-current experiments demonstrating preponderance of conductor-motion-induced quenches near winding's

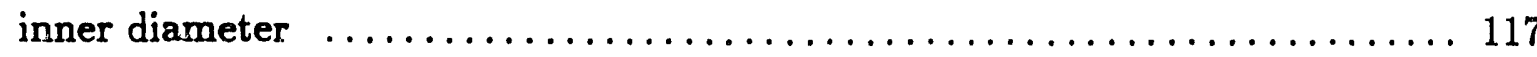

Figure 5.12: a) Quench and b) disturbance histories for a 24-layer solenoid wound with $150-\mathrm{MPa}$ conductor tension $\ldots \ldots \ldots \ldots \ldots \ldots \ldots \ldots$

Figure 5.13: a) Quench and b) disturbance histories for a 24-layer solenoid wound with $150-\mathrm{MPa}$ conductor tension $\ldots \ldots \ldots \ldots \ldots \ldots \ldots \ldots$

Figure 5.14: Friction force/displacement diagram for a sliding pair 
consisting of two Formvar-insulated composite superconductors

Figure 6.1: Conductor-motion model

Figure 6.2: Static displacement $v s$ applied friction coefficient $\ldots \ldots \ldots \ldots 130$

Figure 6.3: Conductor-motion model during stick portion of the stick-slip cycle

Figure 6.4: Conductor-motion model during slip portion of the

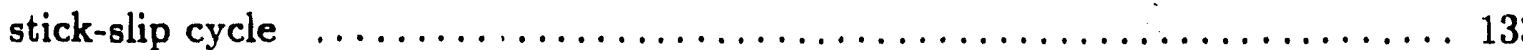

Figure 6.5: Schematic representation of the relaxation modulus, $E_{r}(t, T)$, for a typical viscoelastic polymer $v s$ a temperature of $\log ($ time $)$ axis $\ldots \ldots 138$ Figure 6.6:Viscoelastic, friction-velocity-temperature data for an

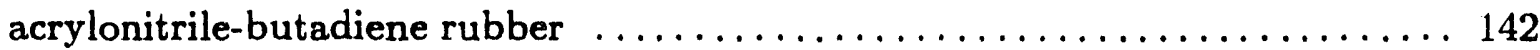

Figure 6.7: Schematic representation of viscoelastic friction model $\ldots \ldots \ldots 144$ Figure 6.8: Grosch's acrylonitrile-butadiene data replotted as friction

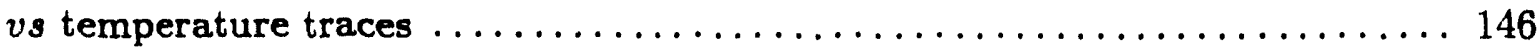

Figure 6.8: Friction vs velocity trace for soft (Durometer 30) silicone rubber against AISI 316 stainless steel at 77 and $293 \mathrm{~K} \ldots \ldots \ldots \ldots \ldots$

Figure 6.10: Time-dependent hardness values and velocity friction coefficients for two compositions of Epon-815 epoxy $\ldots \ldots \ldots \ldots \ldots \ldots \ldots \ldots$

Figure 6.11: Static friction coefficient $v s$ stick time for a 133-phr Epon

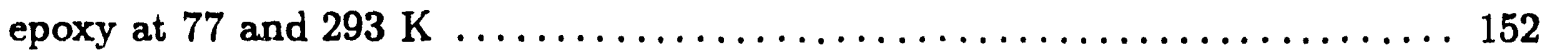

Figure 6.12: Friction coefficient $v$ s sliding speed for an indium disk slid against AISI 316 stainless steel pins at $4.2,77$, and $293 \mathrm{~K} \ldots \ldots \ldots \ldots \ldots 155$

Figure 6.13: Friction-velocity-temperature correlation for lauric-acid-lubricated copper-copper sliding pair $\ldots \ldots \ldots \ldots \ldots \ldots \ldots \ldots$

Figure 6.14: Friction vs temperaiture traces for octanoic-, lauric-, and stearic-acid-lubricated copper-copper pairs $\ldots \ldots \ldots \ldots \ldots \ldots \ldots \ldots$

Figure 6.15: Friction vs velocity traces for stearic-acid-lubricated

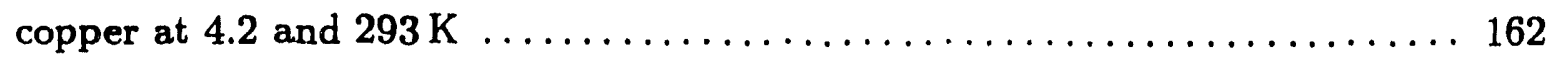

Figure 6.16: Friction $v s$ velocity trace for a mercury disk slid against

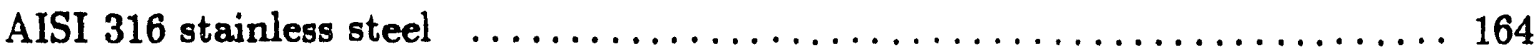


Figure 6.17: Stiffness model for short conductor segments $\ldots \ldots \ldots \ldots \ldots 171$

Figure 7.1: Occurrence of tensile and compressive radial stresses

in an isotropic, radially-connected, infinite-length solenoid $\ldots \ldots \ldots \ldots \ldots 184$

Figure 7.2: Insert coil dimensions (in $\mathrm{mm}) \ldots \ldots \ldots \ldots \ldots \ldots \ldots \ldots$

Figure 7.3: Insert coil load line in 6.5- $\mathrm{T}$ background central induction $\ldots . .189$

Figure 7.4: Thermal-contraction-induced radial stress $v s$ radial position . . 196

Figure 7.5: Winding-tension-induced radial stress $v s$ radial location $\ldots \ldots \ldots 8$

Figure 7.6: Cumulative radial stress at coil midplane $v s$ radial location

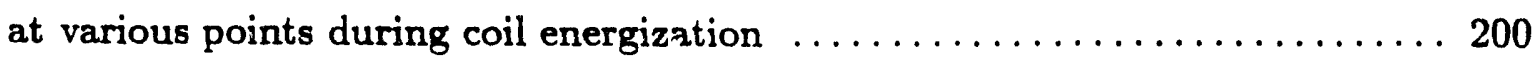

Figure 7.7: Test sequences used during insert coil energization $\ldots \ldots \ldots \ldots 203$

Figure 7.8: Schematic diagram showing details of AE sensor mounting $\ldots 205$

Figure 7.8: Insert coil inductive bridge circuitry $\ldots \ldots \ldots \ldots \ldots \ldots \ldots$

Figure 7.10: Energization diagram showing operating conditions

immediately preceeding insert coil quench $\ldots \ldots \ldots \ldots \ldots \ldots \ldots \ldots$

Figure 7.11: AE/voltage traces observed during second coil quench

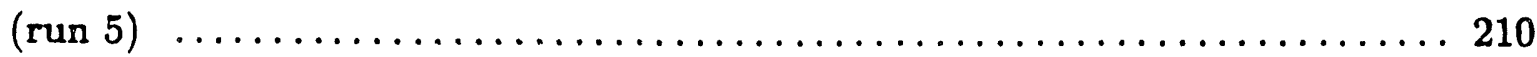

Figure 7.12: AE/voltage traces observed during first coil quench (run 1) .. 212

Figure 7.13: Applied friction coefficient at the end of each conductor layer

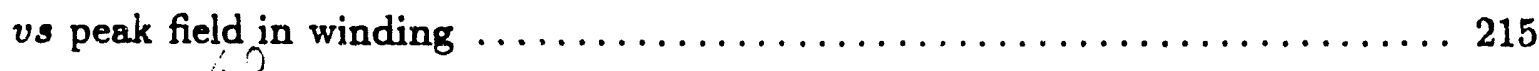

Figure A.1: a) Axial and b) Radial components of the magnetic induction in $\mathrm{T}$ at various locations in the insert coil . . . . . . . . . . . 224

Figure A.2: a) Axial and b) Radial components of the magnetic induction in $\mathrm{T}$ at varic $s$ locations in the insert coil $\ldots \ldots \ldots \ldots \ldots \ldots \ldots 225$

Figure A.3: a) Axial and b) Radial components of the magnetic induction in $\mathrm{T}$ at various locations in the insert coil $\ldots \ldots \ldots \ldots \ldots \ldots \ldots$

Figure B.1: Model conductor used for evaluating insert coil's orthotropic mechanical properties $\ldots \ldots \ldots \ldots \ldots \ldots \ldots \ldots \ldots \ldots \ldots \ldots$ 


\section{List of Tables}

Table 2.1: Comparison of geometric winding parameters for cryostable

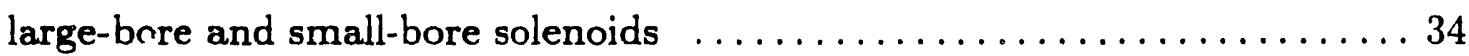

Tabl 3.1: Proposed disturbance energy spectrum $\ldots \ldots \ldots \ldots \ldots \ldots \ldots 1$

Table 4.1: 10-s Brinell hardness values in MPa for materials tested

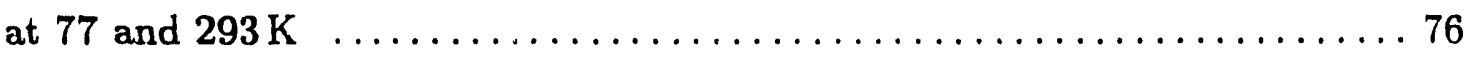

Table 4.2: Brinell hardness values for varous polymeric materials

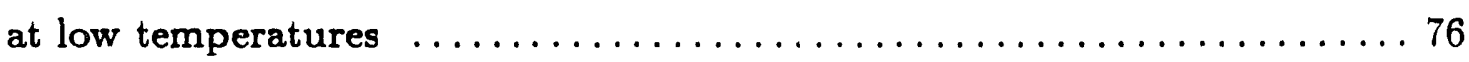

Table 4.3: Test results for the unfilled polymer pins $\ldots \ldots \ldots \ldots \ldots \ldots 8$

Table 4.4: Test results for the fabric- and particle-filled polymer pins $\ldots 83$

Table 7.1: Insert coil conductor specifications $\ldots \ldots \ldots \ldots \ldots \ldots \ldots \ldots$

Table 7.2: Insert coil specifications $\ldots \ldots \ldots \ldots \ldots \ldots \ldots \ldots \ldots \ldots \ldots$

Table 7.3: Insert-coil model's materials properties $\ldots \ldots \ldots \ldots \ldots \ldots \ldots 1$

Table 7.4: Test coil charging sequences $\ldots \ldots \ldots \ldots \ldots \ldots \ldots \ldots \ldots \ldots$

Table 7.5: Magnitude and location of quench inducing conductor motions 212

Table B.1: Physical dimensions for the NbTi winding model .......228

Table B.2: Constituent properties for the model zonductor . . . . . . 229

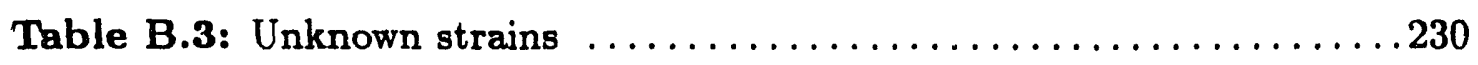

Table B.4: Equivalent orthotropic mechanical properties $\ldots \ldots \ldots \ldots \ldots 231$

Table B.5: Equivalent orthotropic thermal contractions $\ldots \ldots \ldots \ldots \ldots 231$ 


\section{NOMENCLATURE}

This is a list of standard symbols used throughout the thesis. Any other symbols are specified where they are used.

$a_{1} \quad$ - solenoidal winding inner radius

$a_{2} \quad$ - solenoidal winding outer radius

$a_{T} \quad$ - WLF time-temperature superposition shift factor

$A$ - cross-sectional area

$A_{r} \quad$ - real area of contact between sliding surfaces

$b \quad$ - solenoidal winding half length

$B$ - Brinell hardness

$B \quad$ - magnetic flux density

$B_{0} \quad$ - magnetic flux density at the center of a solenoidal winding

$B_{w} \quad$ - peak magnetic flux density in a superconducting winding

$B_{r} \quad$ - radial component of magnetic flux density

$B_{z} \quad$ - axial component of magnetic flux density

$C_{w} \quad$ - winding heat capacity

$d$ - wear scar diameter

$d \quad$ - indentation diameter

$D \quad$ - indentor diameter

E - elastic modulus

$E_{r} \quad$ - radial elastic modulus of superconducting winding

$E_{t} \quad$ - circumferential elastic modulus of superconducting winding

$E_{z} \quad$ - axial elastic modulus of superconducting winding

$E_{r}(t, T)$ - stress-relaxation modulus

$\varepsilon \quad$ - energy

$\varepsilon_{a} \quad-$ thermal activation energy

$\varepsilon_{a e} \quad$ - energy content of acoustic emission signal

$\varepsilon_{q} \quad$ - energy needed to quench a supreconducting winding

$e_{q} \quad-$ quench energy per unit winding volume

$F_{f} \quad-$ friction force

$F_{r} \quad$ - radial contact force

$F_{z} \quad$ - axial force

$f_{r} \quad$ - radial force per unit conductor length

$f_{r}^{\prime} \quad$ - radial electromagnetic force per unit conductor length

$f_{z} \quad$ - axial force per unit conductor length

$g(t)$ - disturbance energy input

$h(t) \quad$ - convective heat transfer coeficient

$I_{c} \quad$ - critical current of superconducting winding 


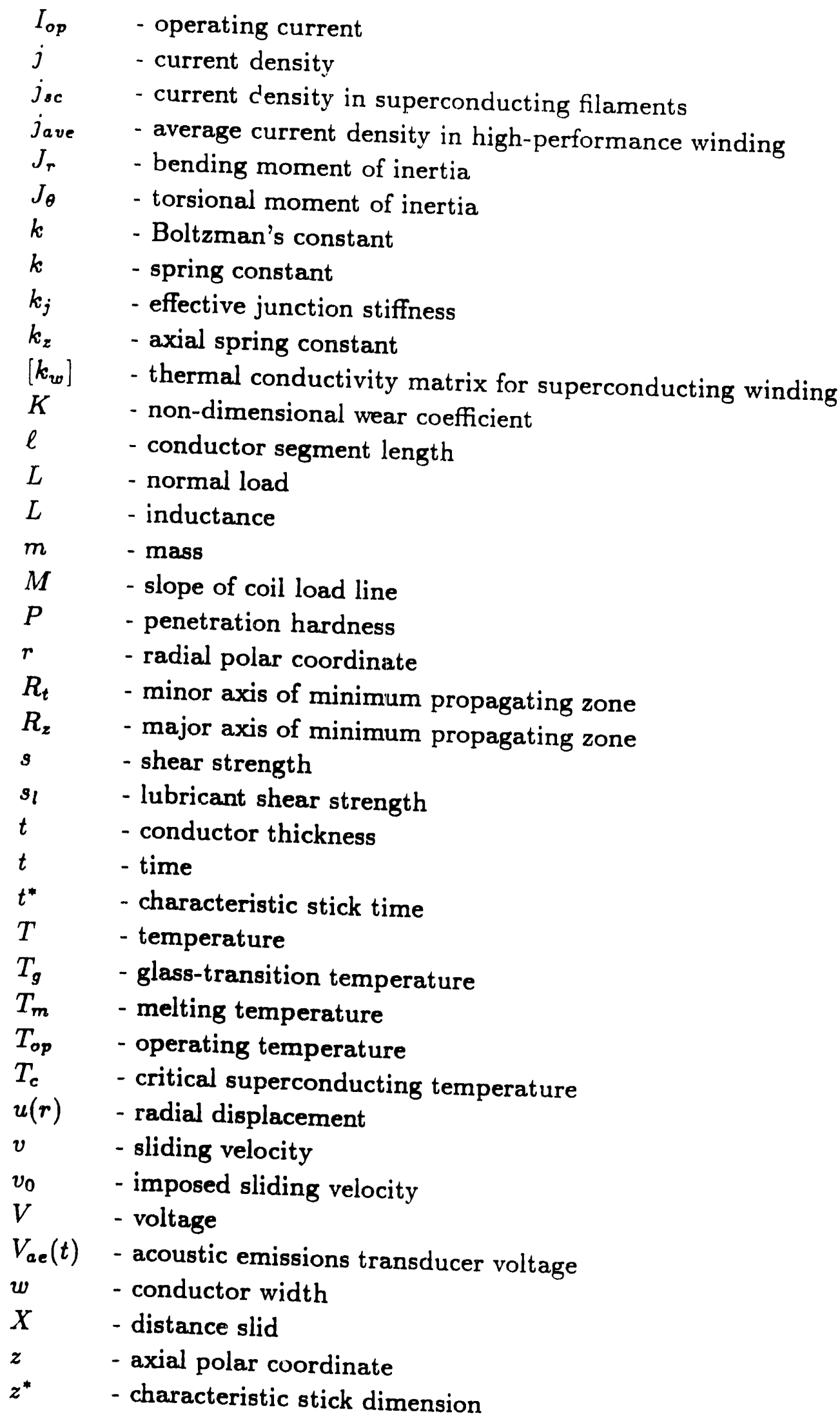




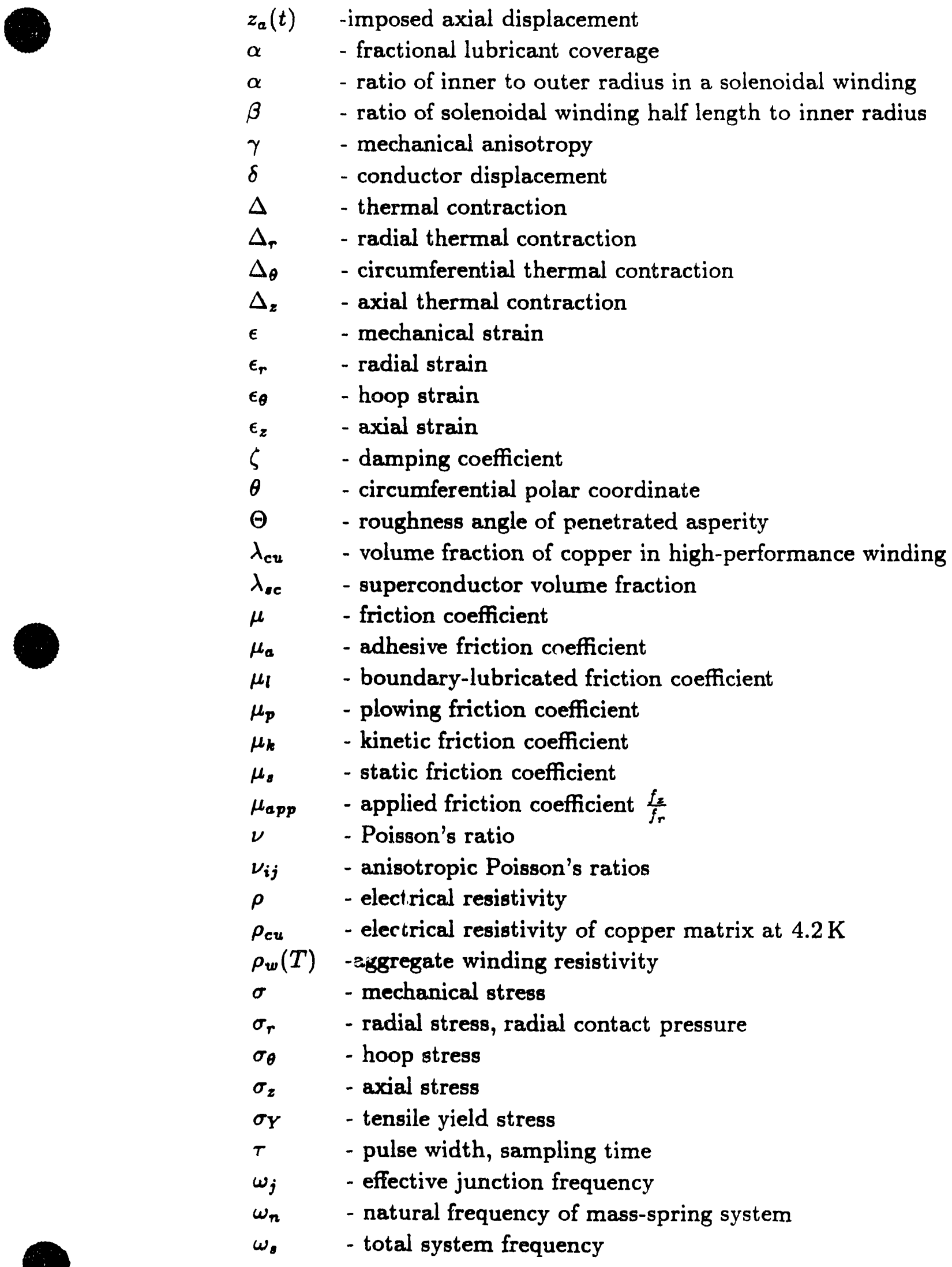




\section{Introduction}

Superconducting magnets are capable of producing very intense magnetic fields while dissipating virtually no electrical power; however, because superconductivity only occurs near absolute zero the magnets must be maintained at very low temperatures to achieve these benefits. This low temperature is generally achieved by immersing the magnet in a liquid helium bath which boils at $4.2 \mathrm{~K}$ at atmospheric pressure.

The heat capacity of a superconducting winding at $4.2 \mathrm{~K}$ is very low, typically less than $10^{-3}$ of its room-temperature value. Consequently, its temperature is strongly affected by relatively minute, highly localized, mechanical-energy disturbances which occur as the magnet is energized. Frictional heating, produced by the rapid slip of conductors against each other, is one example of this sort of disturbance. The energy dissipated during a di urbance is frequently capable of raising the local conductor temperature to above its superconducting transition temperature. The resulting transition between the conductor's superconducting and normal states is known in superconducting parlance as a quench. Hence, these abrupt disturbances pose a severe threat to the magnet's operational stability. The effects of frictional-energy-disturbances on the stability of dry-wound, high-performance superconducting solenoids will be examined.

Chapter 2 introduces the phenomenon of superconductivity and reviews the general characteristics of superconducting solenoids. Chapter 3 characterizes abrupt mechanical disturbances as the primary source of thermal perturbation in highperformance magnets. A semi-quantitative analysis of the energy needed to quench a superconducting winding is derived, and a qualitative explanation of the electromagnetic forces responsible for these mechanical disturbances is developed. Chapter 4 examines the cryogenic-temperature friction and wear of polymer vs metal pairs and concludes that the adhesion theory remains valid for interpreting lowtemperature sliding phenomena. The adhesive friction theory is subsequently used as a guide for evaluating frictionally-induced quenches. 
Chapter 5 focuses on conductor-motion-induced quenches and reviews the contemporary understanding of conductor motion modelling. Two approaches have been developed thus far in an attempt to minimize the effect of these conductor motion disturbances on the magnet's thermal stability. One is to experimentally investigation all potential superconductor-insulator pairs in an attempt to identify the most favorable frictional characteristics. The other is based on theoretical analyses of the magnet's operating stresses and attempts to control motion by regulating the magnitude of the forces transmitted between conductors.

Chapter 6 examines the prospects for achieving stable sliding at cryogenic temperatures. Classical stick-slip friction theories are reviewed. The material conditions necessary for sliding stability are thenretically examined, and structural models are developed for predicting the extent of wire motion in dry-wound solenoids. These structural models are subsequently used in Chapter $T$ to design and analyze the performance of a small superconducting solenoid. The magnet's performance suggests that structural methods are a potentially riable approach for the design of stable dry-wound solenoids.

Chapter 8 summarizes the thesis' major contributions and suggests avenues for further investigation. 


\section{Superconducting magnet technology}

In this chapter the phenomenon of superconductivity is introduced. The composition and critical properties of composite superconducting wires are discussed and potential applications for superconducting magnets are presented. Various approaches for the design of superconducting solenoids are introduced. Particular emphasis is placed on high-performance solenoids, that is, solenoids designed for minimal volume and highly-efficient superconductor utilization. The chapter concludes with a discussion of the design issues presently affecting high-performance magnets and outlines the approach used in this thesis to confront those problems.

\subsection{Superconductivity}

\subsubsection{Phenomenology}

Superconductivity is a phenomenon whereby certain materials become perfect conductors of electricity when cooled to sufficiently low temperatures. A superconductor's electrical resistance decreases gradually as it is cooled, down to a point, known as the material's critical superconducting temperature, $T_{c}$, where its resistance, and hence power dissipation, disappear entirely. The critical temperatures for most commercial superconductors are very low, often only a few Kelvin. The low temperature needed to maintain the superconducting state is usually obtained by submersing the superconductor in a liquid helium bath, boiling at atmospheric pressure at $4.2 \mathrm{~K}$.

The superconducting state is finite: it is bounded not only by temperature, but also by the conductor's electrical current density, $j$, and the ambient magnetic field, $H$. An increase in any one of the material's critical superconducting properties produces a corresponding decrease in the other two. The combinations of the conductor's critical properties in $j-H-T$ space define what is known as the material's critical surface. Superconductivity prevails below this surface and normal resistivity everywhere above it. The objective of a successful magnet design, then, 
is to ensure that the entire conductor remains in the superconducting state during magnet operation.

\subsubsection{Commercial, composite superconducting wires}

Presently, the principal application of superconducting materials is in the construction of high-field, high-current-density electromagnets. Before a superconductor can be incorporated into a magnet winding, it must first be fabricated into a wire. Among the thousands of superconducting compounds that have been identified, only the alloys and intermetallic compounds of niobium are regularly used in commercial superconducting wires.

Conductors based on the $\mathrm{NbTi}$ alloy were first fabricated in 1965 and have since become the "workhorse" of the superconducting industry. Although NbTi has somewhat limited field and current density capabilities, it has good ductility, and is amenable to conventional wire fabrication techniques. The second most frequently used class of conductors is based on $\mathrm{Nb}_{3} \mathrm{Sn}$, an intermetallic niobiumtin compound. Because of their higher field capabilities, $\mathrm{Nb}_{3} \mathrm{Sn}$ conductors are necessary for applications requiring magnetic flux densities above $\sim 12 \mathrm{~T}$. (As a point of reference, the magnetic induction density due to the earth's magnetic field is $\sim 5 \times 10^{-5} \mathrm{~T}$.) The more widespread use of $\mathrm{Nb}_{3} \mathrm{Sn}$ conductors is limited by the $\mathrm{Nb}_{3} \mathrm{Sn}$ 's inherent brittleness, the elaborate processing steps needed to form the conductor, and the conductor's marked sensitivity to mechanical strain; $\mathrm{Nb}_{3} \mathrm{Sn}$ conductors are easily damaged, and their critical properties are severely reduced at mechanical strains above $\sim 0.003{ }^{[1]}$ The winding models developed during this thesis will be restricted to $\mathrm{NbTi}$ conductors because of the analytical complexity of characterizing $\mathrm{Nb}_{3} \mathrm{Sn}$ conductors.

Figure 2.1 shows a cross-section of a typical commercial superconductor; it consists of fine ( $\sim 50 \mu \mathrm{m}$ diameter $)$ NbTi filaments imbedded in a high conductivity copper matrix. The copper matrix serves several important functions in this composite. First, it serves as a carrier for the superconducting filaments. Small diameter filaments are necessary to prevent flux jumping, a combined thermal/electromagnetic instability which renders larger diameter filaments unusable; the adiabatic theory of flux jumping shows that a pure $\mathrm{NbTi}$ wire will have to be $\leq 200 \mu \mathrm{m}$ thick to 

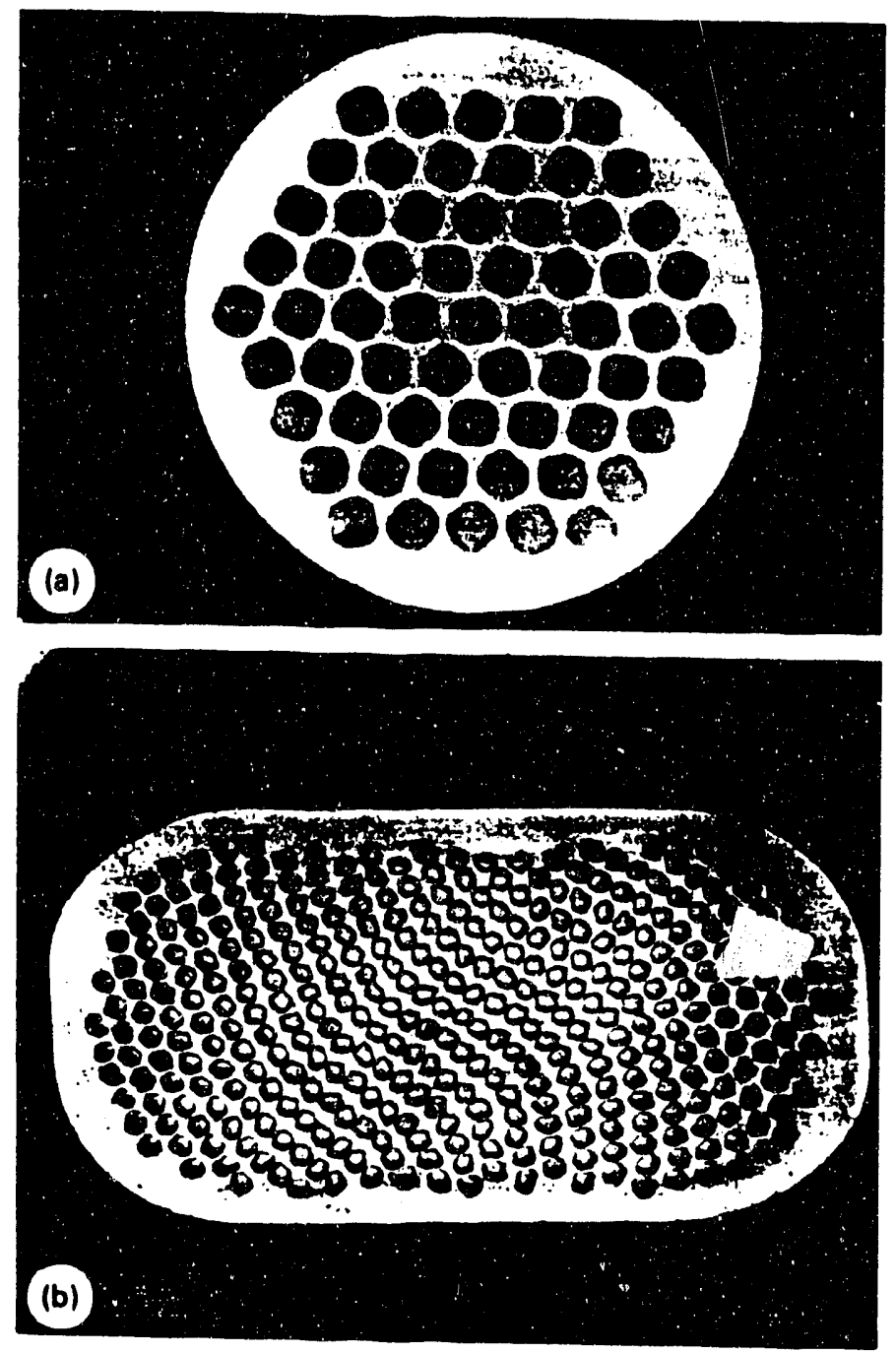

Figure 2.1: Cross-sectional view of commercial, composite superconducting wires, consisting of fine NbTi filaments imbedded in a high conductivity copper matrix. a) Circular and b) rectangular cross-sections. Figure from [2]. 
guarantee freedom from spontaneous flux jumping. ${ }^{[3]}$ By themselves, such small diameter filaments would be impractical to process, however, by combining up to several thousand filaments into a single wire their manufacture is greatly simplified. Second, copper is very ductile, hence it is easily coprocessed with the NbTi. Third, the electrical resistivity of copper near absolute zero is $\sim 2000 \times$ less than the filament's normal state resistivity. ${ }^{[3]}$ If a superconducting-normal state transition does occur, the conductor's current shifts to the copper matrix, effectively reducing the local ohmic heating. Lastly, the copper's thermal conductivity is $\sim 3500 \times$ larger than that for $\mathrm{NbTi}$; this helps to inhibiting the formation of local hot spots by maintaining a more uniform temperature along the conductor's length. ${ }^{[3]}$ The ratio of copper to superconductor in a composite wire can range from about $1: 1$ in a high-current density conductor to $20: 1$ (or more) in cryogenically stabilized wires. ${ }^{[4]}$

Superconducting wires can be produced in a variety of cross-sectional geometries; the two most common are rectangular and round. Round wires have traditionally been used because they are easier to manufacture to high tolerance by conventional wire drawing techniques. However, the use of rectangularly cross-sectioned conductors is becoming increasingly popular. Rectangular wires are easier to wind and offer higher conductor packing densities.

\subsection{Superconducting magnet applications}

Superconducting magnets offer two key advantages over conventional electromagnets. First, they are capable of operating at much higher overall current densities; this allows for a dramatic reduction in the magnet's overall dimensions, typically lowering the magnet's initial capital cost. Second, during d.c. operation a superconducting magnet's electrical energy dissipation can be made vanishingly small, thus resulting in lower operating costs. The largest drawback to superconducting magnets is the relatively modest cryogenic refrigeration power needed to maintain the magnet's near-zero operating temperature.

Superconducting magnets are well suited to applications involving large-volume, high-intensity magnetic fields. As the volume and intensity of the required magnetic field increases, the superconductors' relatively modest refrigeration requirements soon outweigh the tremendous electrical power dissipation required to run 
a comparably-sized, water-cooled, resistive electromagnet. Similarly, because of the uniform operating conditions afforded by their cryogenic environment, superconducting magnets are favored in applications where extremely stable and highly homogeneous magnetic fields are required.

Several potential applications for superconducting magnets have been proposed to date; some have been implemented, and several more are under active investigation. These applications include, but are not limited to the following:

1) plasma confinement for the controlled release of thermonuclear fusion energy.

2) magnetohydrodynamic (MHD) energy conversion for the direction of thermal to electrical energy.

3) superconducting magnetic energy storage (SMES) rings for load-leveling at commercial power plants.

4) magnetically levitated vehicles.

5) MHD ship-based propulsion systems.

6) particle accelerators for high-energy physics experiments.

7) bubble chambers and other particle detectors.

8) windings for compact, high-torque d.c. motors and for high-power-output a.c. generators.

9) laboratory research magnets.

10) nuclear magnetic resonance (NMR) spectrometers for examining the fundamental properties of atomic nuclei.

11) magnetic resonance imaging (MRI) magnets for the non-invasive medical examination of soft body tissues.

\subsection{Solenoidal mangets}

Each of the applications listed in $\$ 2.2$ requires a slightly different design approach and winding configuration. One of the more widely used configurations is the solenoidal winding. Given a choice, solenoidal construction is preferred because it is the easiest configuration to design and manufacture. Solenoids usually con- 
sist of a single conductor, wound autocyclically on a cylindrical coil form. These axi-symmetric magnets are manufactured by rotating the coil form about its axis while slowly feeding the conductor from one end of the coil form to the other and back again. An even number of layers is generally used, so that the input current lead and output lead can attach to the same end of the winding; this simplifies the magnet's connection to an external current supply.

Figure 2.2 presents a cross-sectional sketch of a simple solenoidal winding of inner radius, $a_{1}$, outer radius, $a_{2}$, and length, $2 b$. Because the bore of the magnet is often the only dimension known during the preliminary stages of a magnet design, it is convenient to normalized the winding geometry with resp'sct to $a_{1}$, the coil's inner radius.

$$
\begin{aligned}
& \alpha=\frac{a_{2}}{a_{1}} \\
& \beta=\frac{b}{a_{1}}
\end{aligned}
$$

The normalized winding geometry can then be adjusted to satisfy the following equation which relates the solenoid's central induction, $B_{0}$, to the rest of the major winding parameters. ${ }^{[5]}$

$$
\begin{aligned}
B_{0} & =\mu_{0} j_{s c} \lambda_{s c} a_{1} F(\alpha, \beta) \\
F(\alpha, \beta) & =\beta \ln \frac{\alpha+\left(\alpha^{2}+\beta^{2}\right)^{1 / 2}}{1+\left(1+\beta^{2}\right)^{1 / 2}}
\end{aligned}
$$

In this equations, $\mu_{0}$ is the permittivity of free space, $\lambda_{s c}$ the volume fraction of superconductor in the winding, and $j_{s c}$ the superconductor current density. $F(\alpha, \beta)$ is a geometric field factor that is derived by dividing the winding into a large number of "elemental" current loops and integrating their contributions to the on-axis field at the center of the coil.

\subsection{Solenoid magnet design}

During the design of a superconducting solenoid several trade-offs between the various winding parameters are possible. The characteristics usually specified at the start of a design include: the magnet's central induction, $B_{0}$, the desired volume of accessible field, and an allowable field uniformity. While not specified, it is usually assumed that a magnet should cost no more than necessary to guarantee 


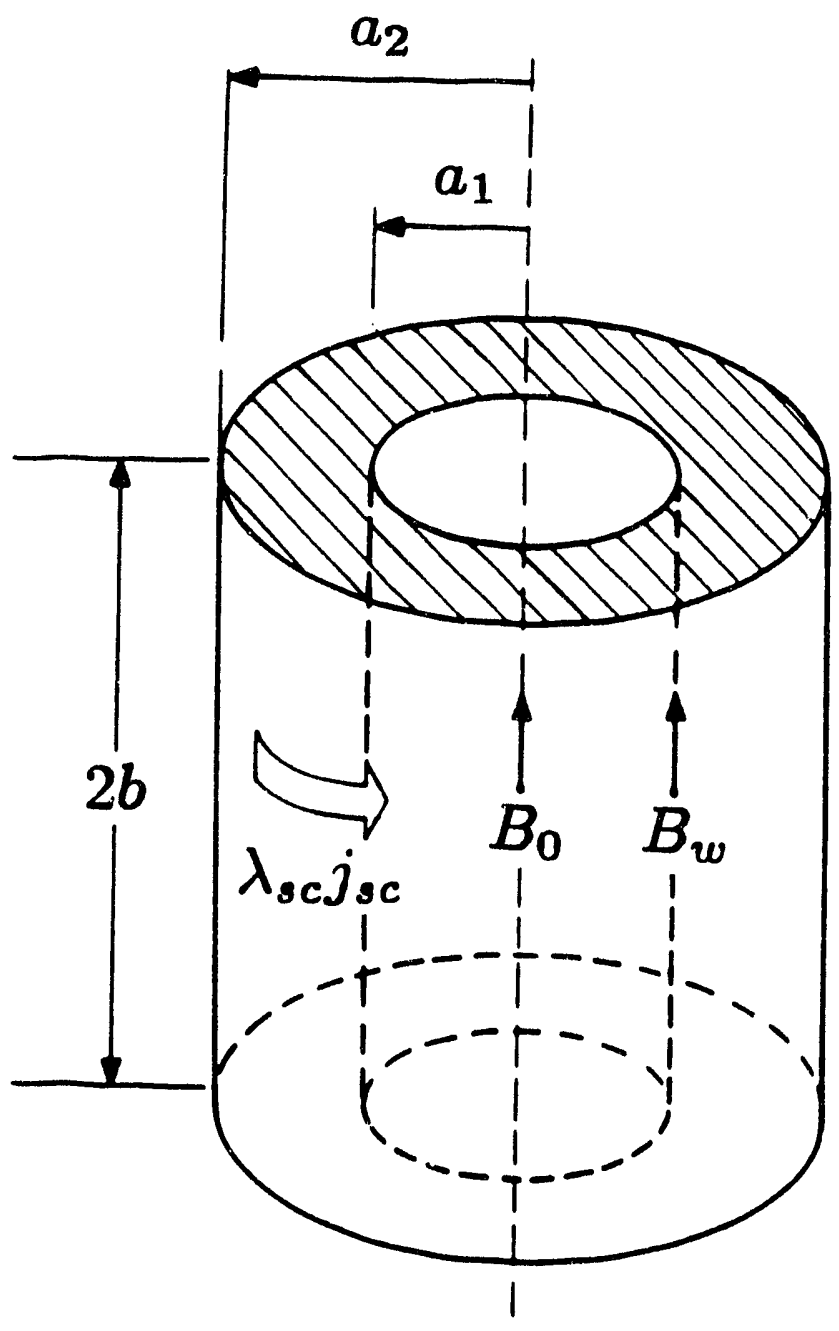

Figure 2.2: Schematic representation of a simple solenoidal winding. 
reliable operation. This trade-off between cost and reliability remains one of the larger impediments to the widespread commercial development of superconducting magnets.

The effect that a magnet's specified characteristics have on its overall design is briefly outlined in the following subsections.

\subsubsection{Winding volume}

The major factor which determines the cost of a superconducting magnet, and its associated hardware, is the winding volume. The magnet volume determines not only the quantity of superconductor required, but also the systern's weight, the size of the facilities needed to site the magnet, and the refrigeration power required to maintain the magnet's operating temperature. A large magnet volume increases the size of the required support structure, hence more heat is conducted into the helium cooling bath.

In terms of the normalized coil dimensions given in Eq. 2.1, the volume $V$ of a solenoidal winding can be expressed:

$$
\begin{gathered}
V=a_{1}^{3} \cdot v(\alpha, \beta) \\
v(\alpha, \beta)=2 \pi\left(\alpha^{2}-1\right) \beta .
\end{gathered}
$$

Because $a_{1}$ enters this expression to the third power, the winding's inner radius is usually selected to be no larger than necessary to accommodate the magnet's accessible "working" field.

\subsubsection{Field uniformity}

In applications, such as NMR spectroscopy, the winding's central magnetic field is typically specified to a uniformity of $\sim 1$ part in $10^{5}$ over a central spherical region, whose diameter is about $1 / 2$ the diameter of the windings inner bore. Starting from this background field, uniformities on the order of 1 part in $10^{8}$ can subsequently be achieved through the use of additional "shim" coils, small windings specifically tailored to enhance the central field uniformity. ${ }^{[6]}$

A solenoid's field uniformity is controlled almost exclusively by its geometric proportions, $(\alpha, \beta)$. Analytical methods based on the Taylor series expansion of Eq. 2.2 about the solenoid's center have been developed to relate the central field 
uniformity to the normalized winding geometry. $[5,7]$

$$
\begin{aligned}
B_{z}(r, \theta) & =B_{0}\left[1+E_{2}\left(\frac{r}{a_{1}}\right)^{2} P_{2}(u)+E_{4}\left(\frac{r}{a_{1}}\right)^{4} P_{4}(u)+\ldots\right] \\
B_{r}(r, \theta) & =B_{0}\left[0+E_{2}\left(\frac{r}{a_{1}}\right)^{2} P_{2}^{\prime}(u)+E_{4}\left(\frac{r}{a_{1}}\right)^{4} P_{4}^{\prime}(u)+\ldots\right] \\
E_{2 n} & =\frac{1}{B_{0}} \frac{1}{(2 n) !} \frac{d^{2 n} B_{z}(z, 0)}{d z^{2 n}}
\end{aligned}
$$

In these equations, $B_{z}$ and $B_{r}$ respectively are the axial and radial components of the central magnetic induction, $r$ and $\theta$ are the conventional radial and spherical angle polar coordinates, and the $E_{2 n}$ coefficients are defined by the standard Taylor series expansion formula. The spatial variations in the magnetic inductions are described respectively by the Legrende Polynomials, $P_{2 n}(u)$, and their derivatives, $P_{2 n}^{\prime}(u)$. ${ }^{[8]}$

To achieve the good field uniformity needed in high homogeneity magnets, several nested solenoids are frequently used; these are designed so that the higher order terms from one of the windings, $E_{2 n}$, can be used to exactly cancel the same, combined higher order terms from the others. Coil segmentation is likewise be used to limit the peak electromagnetic stresses that are generated in the windings during magnet operation. ${ }^{[9]}$

For illustrative purposes, it is generally sufficient to consider the variation in magnetic induction across the magnet midplane to appreciate the effect of winding geometry on field uniformity. The magnetic induction across a solenoid's midplane increases from $B_{0}$ at the center to a maximum value of $B_{w}$ at the winding's inner radius, $a_{1}$. However, because $B_{w}$ cannot be determined analytically recourse must be made to numerical computation to determine the winding's peak magnetic flux density. Figure 2.3 presents the results of this computation and shows parameterized curves of constant $B_{w} / B_{0} v s \alpha$ and $\beta .{ }^{[10]}$ This figure shows that the normalized coil length, $\beta$, has by far the greatest effect on the solenoid's central field uniformity. The effect of $\beta$ on field uniformity is consistent with the infinite-length solenoid approximation; as a solenoid's length increases, its internal field becomes increasingly uniform. 


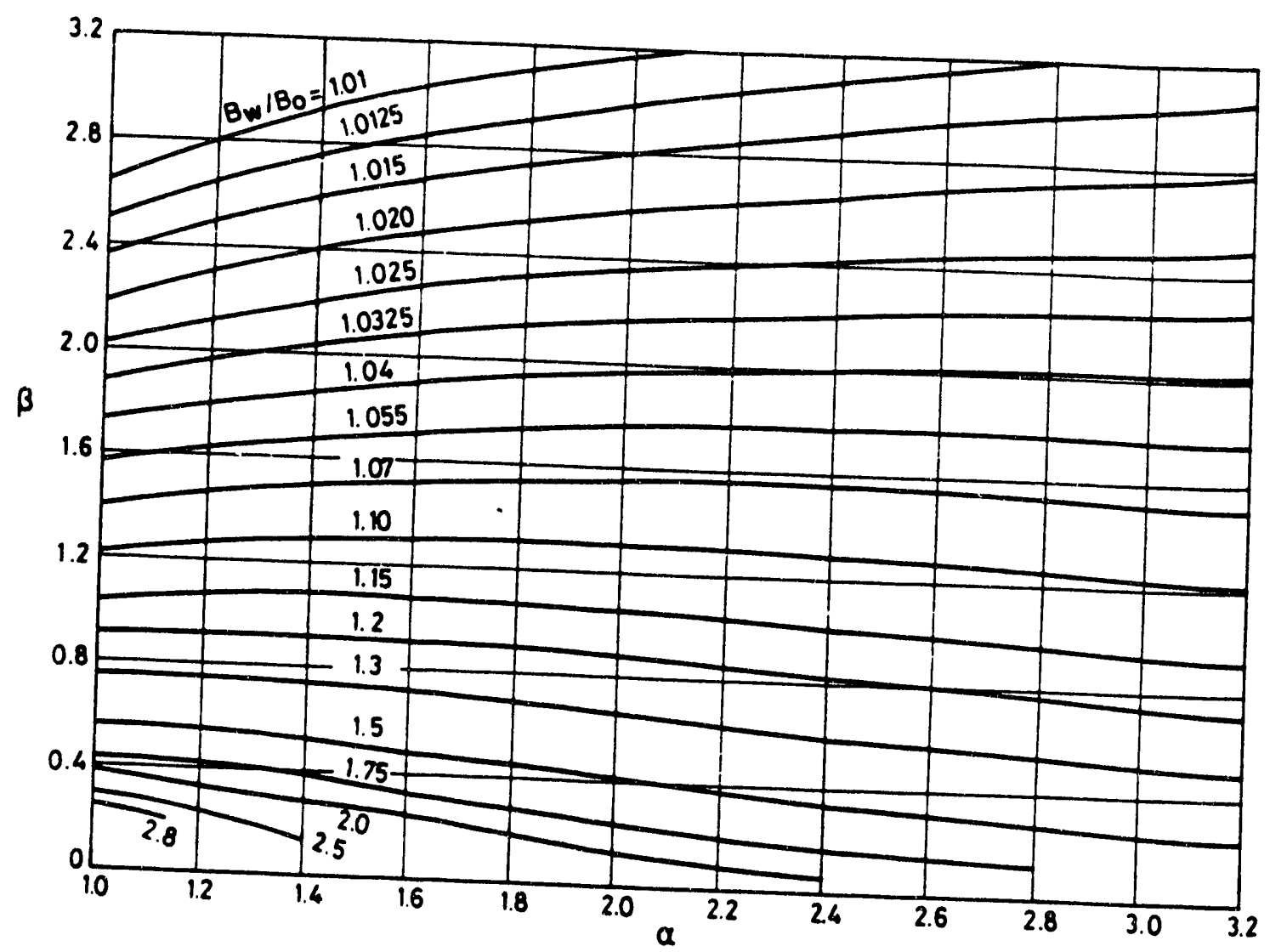

Figure 2.3: Parameterized curves of constant $B_{w} / B_{0}$, maximum to central induction, in a simple solenoid $v s \alpha$ and $\beta$. Figure from [6]. 


\subsubsection{Superconductor current density}

The critical current density in a superconducting solenoid is limited by $B_{w}$, the peak magnetic induction in the windings; this effect is illustrated in Figure 2.4. Figure 2.4 presents a section view of the critical surface diagram for NbTi at $4.2 \mathrm{~K}$, the normal operating temperature for superconducting magnets. The line OP represents the "load line" for a hypothetical solenoid which produces a peak induction of $6 \mathrm{~T}$. As the magnet is energized, its magnetic induction and current density increase together linearly towards the operating point P. From Eq. 2.2, the slope of OP is:

$$
M=\frac{1}{\mu_{0} a_{1} \lambda_{s c} F(\alpha, \beta) \frac{B_{w}(\alpha, \beta)}{B_{0}}} .
$$

The $B_{w}(\alpha, \beta) / B_{0}$ correction term is used to account for the discrepancy between the peak induction in the windings and the winding's central induction. Although few magnets are designed to operate exactly at their critical current density, Figure 2.4 serves to illustrate the effect that the superconductor's critical properties have on a magnet's overall design.

The fact that $B_{w}(\alpha, \beta) / B_{0}>1$ implies that not all of the superconductor's current density goes towards producing "useful" field. However, by keeping $B_{w}(\alpha, \beta)$ close to $B_{0}$ this effect can be minimized. Particularly in short solenoids $(\beta<1)$ a reduction in the quantity of superconductor required to produce a given $B_{0}$ can sometimes be achieved by slightly increasing the coil's length; $B_{w}$ will then be less and the allowable superconductor current density, $j_{s c}$, will increase. Because of the resulting trade-offs between $B_{w} / B_{0}$, the allowable superconductor current density, and the coil volume, the specification of a solenoid's optimum geometry often requires several iterations. This is especially true in nested, multiple coil systems where both the magnitude and location of the peak induction, $B_{w}$, in each winding depends on the geometric configuration of the other coils.

An added advantage to the use of multiple coil systems is their potential for improved superconductor utilization. By winding each coil from a different diameter conductor (assuming a constant operating current between coils) it is possible to maximize the superconductor's current density within each coil to the local magnetic induction; this method uses the much larger current densities available in the 


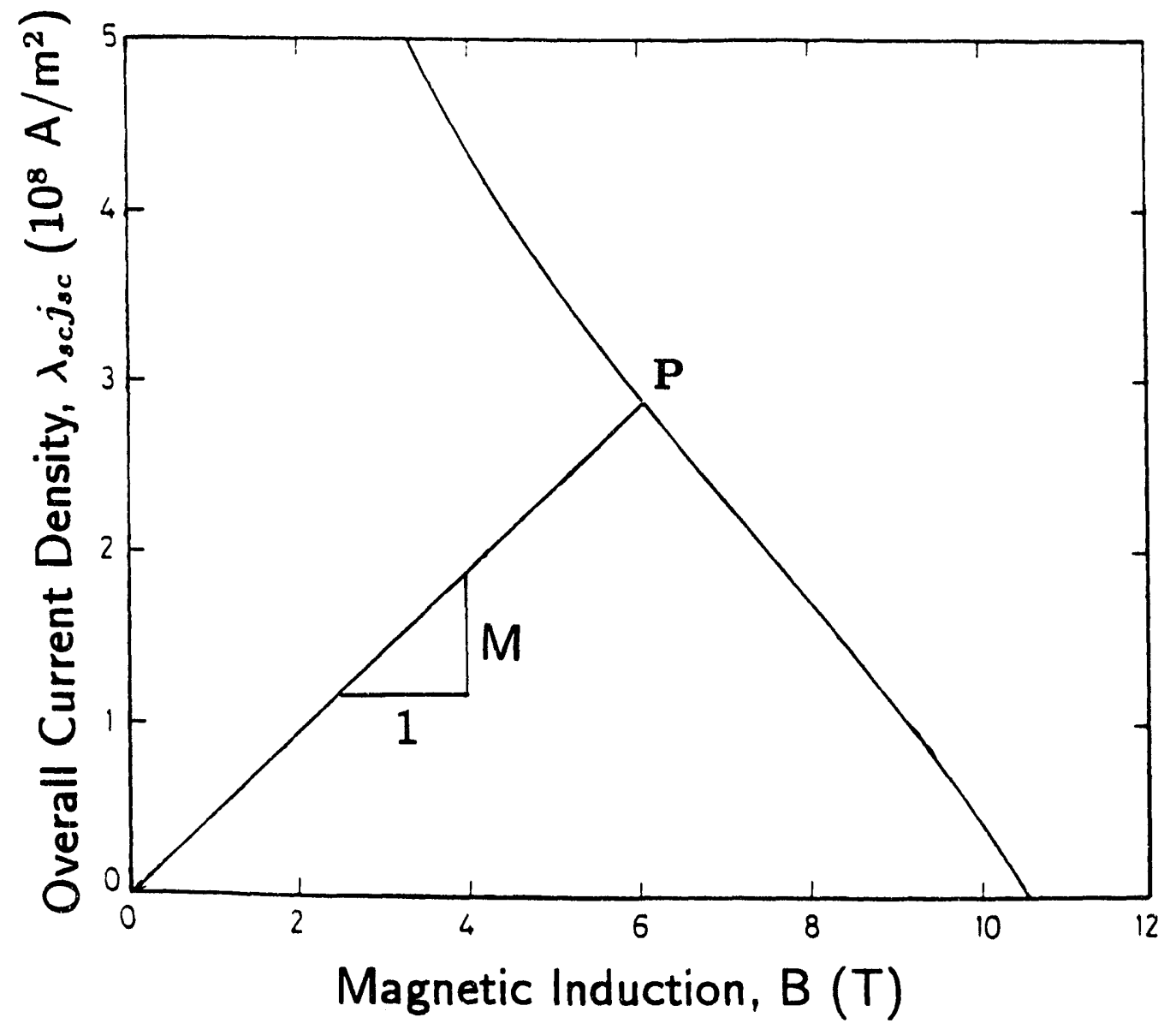

Figure 2.4: Idealized load line and critical superconducting properties for a NbTi winding at $4.2 \mathrm{~K}$. Critical superconducting property data from [6]. 
system's low field regions to reduce the total required quantity of superconductor.

\subsubsection{Superconductor volume fraction}

By far, the parameter which has the largest effect on a magnet's design is its overall current density, $j_{a v e}=j_{s c} \lambda_{s c}$. The maximum allowable superconductor current density, $j_{s c}$, is limited by the winding's peak field, $B_{w}$. By contrast, $\lambda_{s c}$ is usually not directly specified but arises as a consequence of the solenoid's proposed stabilization method.

The electromagnetic energy storage density in a typical $7 \sim 12 \mathrm{~T}$ solenoid is in the range $20-60 \mathrm{MJ} / \mathrm{m}^{3}$, comparable in magnitude to the storage density of a large rotating flywheel. ${ }^{[6]}$ Hence, a key goal in an effective magnet design is to allow for the safe handling of this energy.

Stekly and Zar have proposed two conditions for guaranteeing absolutely stable magnet operation. ${ }^{[11]}$ The first is to use a large proportion of copper in the conductor so that the power dissipation in the event of a quench would be limited to a manageable level. The second is to provide sufficient cryogenic cooling throughout the winding so that superconductivity could be restored even if the entire magnet were to quench. Because of their large cryogenic cooling capacity these magnets are referred to as being completely cryostable. In cryostable magnets, the superconducting volume fraction, $\lambda_{s c}$, is typically around $0.03 .{ }^{[12]}$ The low superconductor volume fraction arises for several reasons. One is the large volume fraction of cooling passages needed for effective cooling. A second is the additional structural material needed to support and insulate the conductors and to form the cooling channel boundaries. A third is the large percentage of copper needed to minimize ohmic heat generation following the quench.

Figure 2.5 presents parameterized curves of constant field factor, $F(\alpha, \beta)$, and constant volume factor, $v(\alpha, \beta)$. The solid line in the figure represents the geometric minimum volume winding capable of achieving each $F(\alpha, \beta)$. Since $v(\alpha, \beta)$ varies roughly as $\alpha^{2}$ the numerical value of the $v(\alpha, \beta)$ along this line increases much more rapidly than does $F(\alpha, \beta)$.

The low superconductor volume fraction required for cryostability is economically acceptable only for very large bore magnets. From Eq. 2.2, the low value of 


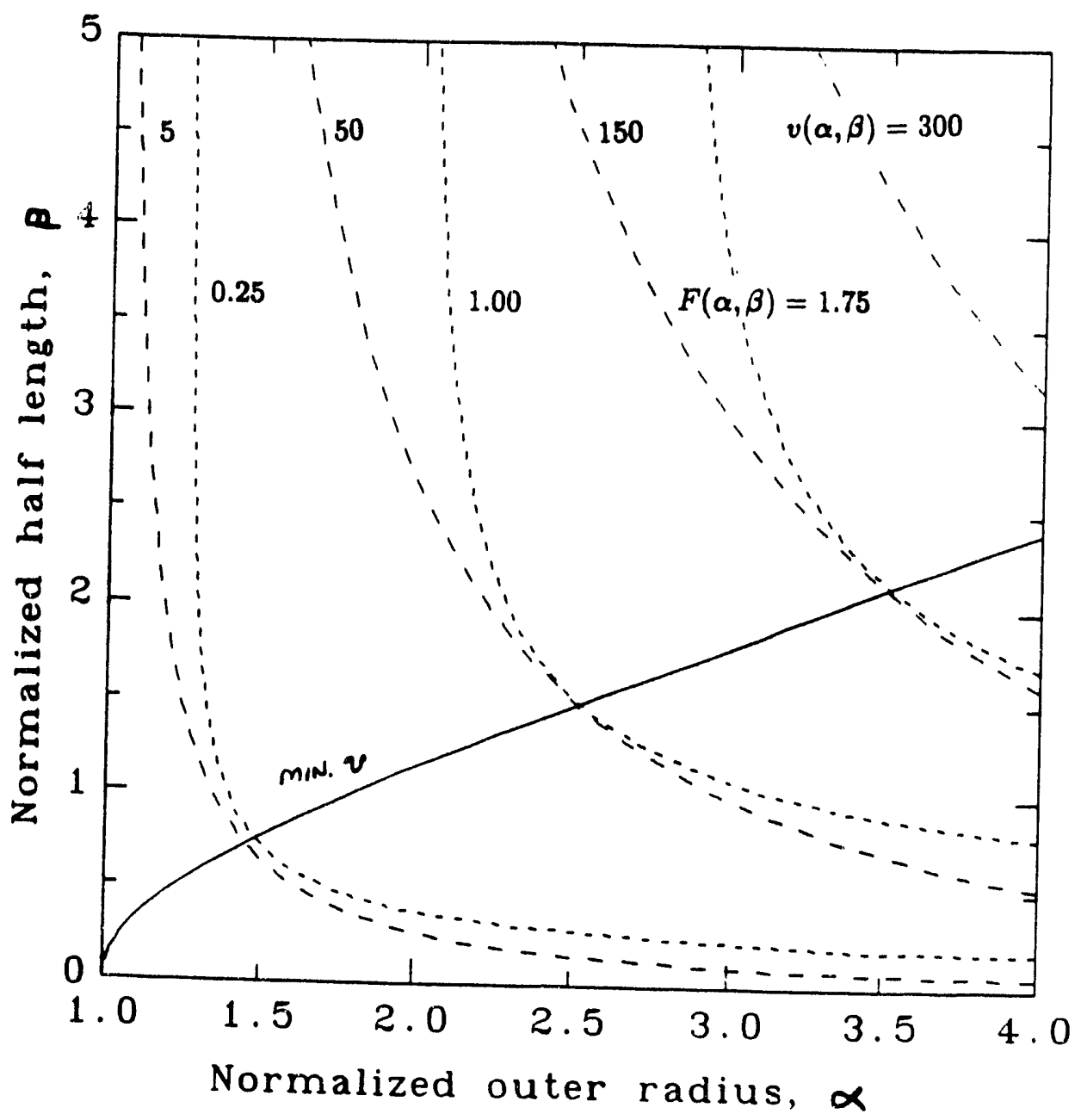

Figure 2.5: Curves of constant $F(\alpha, \beta)$ and constant $v(\alpha, \beta) v s \alpha$ and $\beta$. The solid line in this graph represents the minimum geometric volume required to achieve each $F(\alpha, \beta)$ value. 
$\lambda_{s c}$ necessary for cryostabilization can be traded-off against a large inner radius, $a_{1}$, to maintain fairly moderate values for $F(\alpha, \beta)$ and hence for $v(\alpha, \beta)$. However, for small-bore magnets, because $a_{1}$ is already small, the very large $F(\alpha, \beta)$ and $v(\alpha, \beta)$ values which result, render cryostable small-bore magnets economically infeasible; Table 2.1 presents a numerical example of this effect. The coils in this table are designed using the "minimum volume" curve in Fig. 2.5 to produce a central induction of $6 \mathrm{~T}$ with the superconductor operating at its critical current density. Coil 1 has an inner radius, $a_{1}$, of $1 \mathrm{~m}$ (typical for a particle detector magnet), while Coil 2 has $a_{1}=0.05 \mathrm{~m}$ (typical of a small research solenoid).

Table 2.1: Comparison of geometric winding parameters for cryostable large-bore and small-bore solenoids.

\begin{tabular}{|r|c|c|}
\hline & Coil 1 & Coil 2 \\
\hline Central induction, $B_{0}(\mathrm{~T})$ & 6.00 & 6.00 \\
\hline Superconductor current density, $j_{s c}\left(\mathrm{~A} / \mathrm{m}^{2}\right)$ & $1.9 \times 10^{9}$ & $1.9 \times 10^{9}$ \\
\hline Inner radius, $a_{1}(\mathrm{~m})$ & 1.00 & 0.05 \\
\hline Superconductor volume fraction, $\lambda_{s c}$ & 0.03 & 0.03 \\
\hline Geometric field factor, $F(\alpha, \beta)$ & 0.08 & 1.67 \\
\hline Minimum volume factor, $v(\alpha, \beta)$ & 1.40 & 139.6 \\
\hline Normalized half length, $\beta$ & 0.48 & 2.07 \\
\hline Normalized outer radius, $\alpha$ & 1.21 & 3.42 \\
\hline
\end{tabular}

The very large radial thickness for Coil 2 results because of the low overall current density, $j_{s c} \lambda_{s c}$, assumed in this example. Roughly speaking, a magnet's overall current density starts to have a strong affect on its economics when the winding thickness becomes comparable to the inner radius $(\alpha>2){ }^{[12]}$ Thus, an entirely separate design philosophy has developed to enhance the economic competitiveness of small bore magnets. Under this second design approach the objective is to maximize the overall superconducting current density so as to produce very compact, structurally robust, high-performance magnets.

\subsection{High-performance magnets}

The major difference between cryostable and high-performance windings is the high-performance winding's lack of cryogenic cooling channels. The absence of cooling passages has the added effect of eliminating many the structural materials 
previously required as spacers between the conductors. In fact, because of their circular symmetry, most high-performance solenoids require no other structural support than that provided by the conductors themselves. The conductors thus serve a dual role both as current carrying elements and as structural members. Figure 2.6 presents winding models for magnets constructed from both rectangular and round cross-sectioned conductors. A close packed conductor array is typically used to maximize the conductor volume fraction and to provide good structural integrity.

The lack of cooling passages likewise allows for a drastic reduction of the quantity of copper used in the conductors; far less copper is required to prevent quenchinduced thermal damage than is needed to accommodate thermal recovery. Empirical guidelines have been developed to specify the minimum volume fraction of copper, $\lambda_{c u}$, needed to prevent ohmic-heating-induced thermal damage. ${ }^{[13]}$

$$
\lambda_{c u} \simeq \frac{\lambda_{s c} j_{s c} \sqrt{\varepsilon}}{300}
$$

In this equation, $j_{s c}$ is the superconductor's operating current density in $\mathrm{A} / \mathrm{mm}^{2}$, and $E$ is the coil's stored magnet energy in MJ. Once the total conductor volume fraction $\left(\lambda_{s c}+\lambda_{c u}\right)$ has been determined, this relation can be used to specify the ratio of copper to superconductor used in the conductor; although there is no upper limit to this ratio, a lower limit of $\sim 1: 1$ is typically needed for reliable manufacture and to limit the interconductor voltages during quenching. The total conductor volume fraction typically ranges for about $70 \sim 90 \%$, depending on the size and cross-sectional geometry of the conductor as well as on the insulation thickness. The remainder of the coil consists of insulation and interconductor void spaces.

Applying this high-performance design philosophy to the the small-bore solenoid listed as Case 2 in Table 2.1 with an assumed $\lambda_{s c}$ value of 0.4 produces a much more economical minimum winding volume with $\alpha=1.29, \beta=0.56, F(\alpha, \beta)=0.126$, and $v(\alpha, \beta)=2.30$, a winding volume $1 / 60$ of that required by the original cryostable design. Although most magnets' geometries are dictated by considerations additional to the minimum volume, these hypothetical values serve to illustrate the large reduction in volume possible using a high-performance design. 

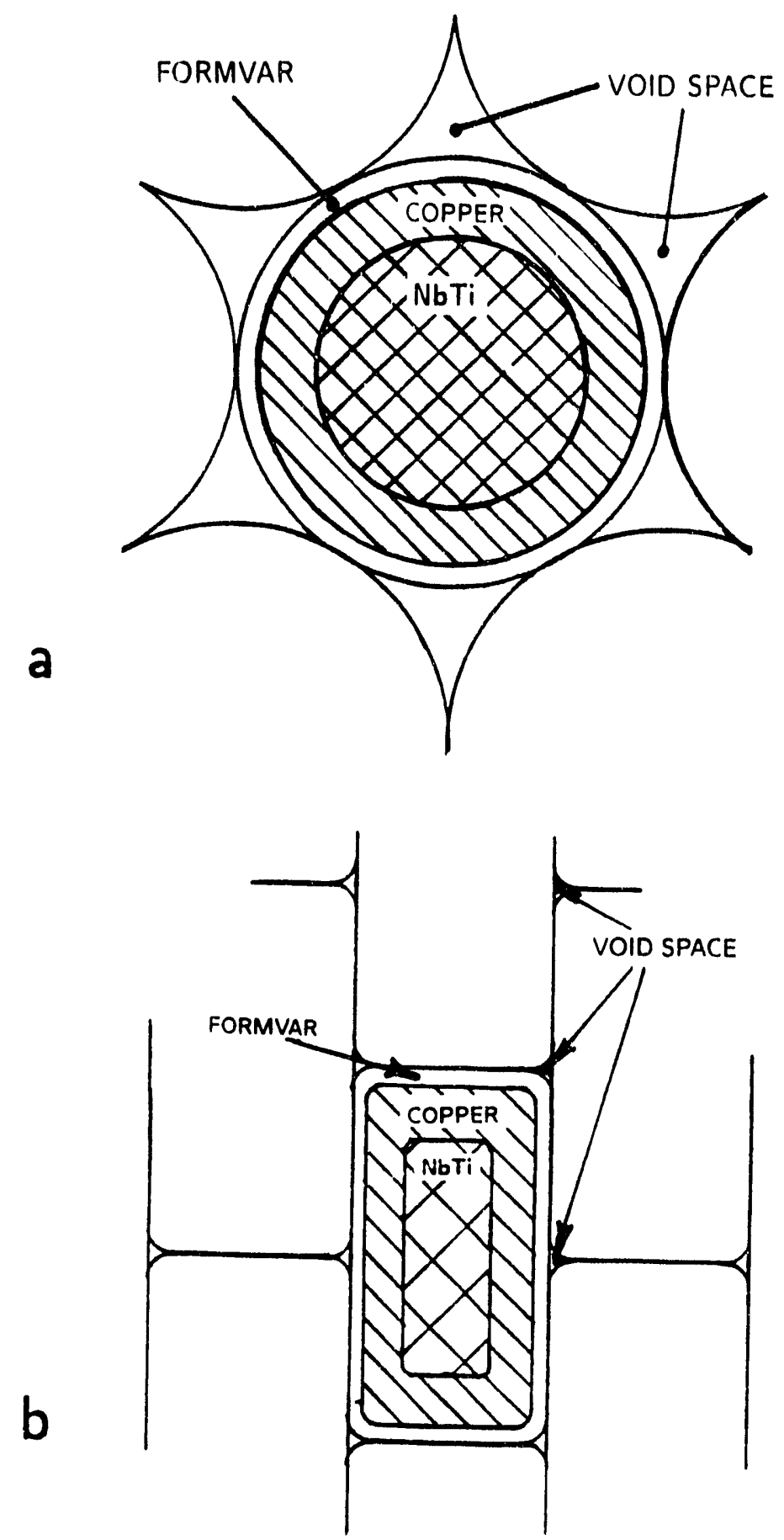

Figure 2.6: Winding models for high-performance magnets constructed from a) circular and b) rectangular cross-sectioned conductors. 


\subsection{Thesis Overview}

The most important driving force favoring high-current-density magnets is performance; the risk is lower reliability. The ultimate goal is to improve performance while maintaining a reasonable degree of reliability.

Presently, virtually all high-performance magnets quench at least once before achieving their rated output. Because only enough copper is used in the winding to prevent excessive normal zone voltages, the maximum temperature rise during a quench can often be several tens of $\mathrm{K}$. Several liters of relatively expensive helium coolant are then required to return the magnet to its designed operating temperature following a quench. For instance, each quench during the qualification tests for the Superconducting Super Collider dipole magnets typically evaporated approximately $100 \sim 500 \ell$ of helium. It frequently requires several attempts to energize a magnet before its design point is reached; this progressive increase towards the magnet's designed operating point is known as training. The worst case scenario occurs when the magnet fails to achieve its designed performance even after numerous charging cycles; this behavior is referred to as degraded performance. Thus, despite their considerable potential for a wide variety of applications, the more widespread commercial acceptance of high-performance magnets has been limited by their lack of absolute reliability.

Considerable research effort has been devoted over the past fifteen years to develop methods for reducing, if not eliminating, the training phenomenon in highperformance magnets. This thesis is a direct continuation of that effort; in particular, it emphasizes methods for reducing conductor-motion-induced frictional-heating disturbances, a primary source of quenches in high-performance solenoids. 


\section{References for Chapter 2}

1. Ekin, J.W. Fatigue and stress effects in $\mathrm{NbTi}$ and $\mathrm{Nb}_{3} \mathrm{Sn}$ multifilamentary superconductors Adv. Cryo. Eng. 24 (1978) 306-316.

2. Wilson, M.N. Superconducting Magnets Clarendon Press, Oxford (1983) p137.

3. Wilson, M.N. Practical superconducting materials, Ch. 2 in Superconductor Materials Science (eds. S. Foner and B.B. Schwartz) Plenum Press, New York (1981) 63-131.

4. Ekin, J.W. Mechanical properties and strain effects in superconductors, Ch. 7 in Superconductor Material Science (eds. S. Foner and B.B. Schwartz) Plenum Press, New York (1981) 455-510.

5. Montgomery, D.B. Solenoid Magnet Design Wiley-Interscience, NY (1969), reprinted 1980 by R.F. Krieger Publishing, Malbar FL.

6. Wilson, M.N. op. cit. pp6-27.

7. Garrett, M.W. Axially symmetric systems for generating and measuring magnetic fields. Part I. J. Appl. Phys. 22 (1951) 1091-1107.

8. Examples of the Legrende polynomials, $P_{2 n}$, are listed in Montgomery, D.B op. cit. Table 8.1, p235.

9. Lontai, L.M. and Marston, P.G. A 100 kilogauss quasi-continuous cryogenic solenoid - part 1 Proc. First Int. Symp. Mag. Tech. (eds. H. Brechna and H.S. Gordon) Stanford (1965) 723-732.

10. Boom, R.W., and Livingston, R.S. Superconducting solenoids Proc. Inst. Radio Eng. (1962) 50 274-285.

11. Stekly, Z.J.J, and Zar, J.L. Stable superconducting coils IEEE Trans. NS-12 (1965) 367-372.

12. Wilson, M.N. op. cit. pp102-104.

13. Bobrov, E.S. Private correspondence (Jan. 1991). 


\section{Mechanical disturbance induced quenches}

Thermal disturbances are the primary cause of quenches in high-performance superconducting magnets. There are several possible sources of thermal perturbation; the most common are localized, energy-dissipative mechanical events which occur during energization because of the magnet's changing stress state. The local energy dissipation during an abrupt disturbances is often capable of quenching the conductor, that is, raising the conductor's temperature to above its critical superconducting temperature. Because no cooling is provided inside a high-performance magnet, the additional ohmic heating introduced by a local conductor quench initiates a thermal runaway that causes the normal zone to spread rapidly throughout the rest of the winding.

The chapter is divided into three sections. The first uses a local heat transfer equation to discuss a conductor's thermal stability in response to various disturbance energy inputs and highlights approximate solutions developed to estimate the disturbance energy necessary for quench initiation. The second section reviews the present understanding of quench initiation in high-performance solenoids. Methods have been devised to mitigate the effects of virtually all of the recognized disturbance sources except for conductor-motion-induced frictional heating. The third section summarizes the experimental measurement techniques used to investigate conductor-motion-induced disturbances.

\subsection{Thermal stability of high-performance magnets}

Stability is a characteristic of steady-state operation. If a system, such as a superconducting magnet, returns to its original operating condition following a perturbation, the steady state is said to be stable. If the system does not return to its original state it is said to be unstable. Sometimes, the system is stable against small perturbations but unstable against large ones; this is an example of metastable behavior. ${ }^{[1]}$

Superconducting magnets can exhibit all three types of behavior, depending 
on the design philosophy used in their construction. As discussed in $\$ 2.4 .4$, the cryostable design approach, which is used to achieve absolutely stable performance. requires a large dilution in the magnet's overall current density, hence, it is not economically feasible for small bore magnets. The best compromise achieved thus far for high-performance magnets has been to design them for metastable operation. this is, so that they operate $0.1 \sim 0.3 \mathrm{~K}$ below the conductor's critical surface; this reduces the magnet's efficiency slightly but provides a small temperature margin to accommodate small scale disturbances that may occur during operation.

\subsubsection{Energy balance for a unit winding volume.}

The ultimate goal of all systems is continuity of operation. Hence, once a magnet's temperature margin has been selected the task becomes one of limiting the peak winding temperature to below this limit. The following power density heat-balance characterizes the thermal behavior of a unit conductor element.

$$
C_{w} \frac{d T}{d t}=\nabla \cdot\left(\left[k_{w}\right] \nabla T\right)+\rho_{w}(T) j_{a v e}^{2}(t)-\left(\frac{P}{A}\right) h(T)+g(t)
$$

The left hand side of this equation represents the change in the stored thermal energy of the winding element; $C_{w}$ is the winding's heat capacity. The first term on the right hand side represents heat flow into the element via conduction; $\left[k_{w}\right]$ is the winding's thermal conductivity matrix and $T$ the conductor temperature. $k_{w}$ is different along each of the principle axes because of the anisotropic arrangement of the winding's constituents. The second term represents joule heating; $\rho_{w}(T)$ is the winding's equivalent resistivity and $j_{a v e}(t)$ the overall, or average, current density in the winding. The third term represents convective cooling; $P / A$ is the ratio of the conductor's wetted perimeter to its cross-section area and $h(T)$ is the convective heat transfer coefficient. Because of the absence of cooling passages in high-performance magnets $h(T) \equiv 0$ in the winding's interior. Any heat produced in the magnet must first be conducted to the winding's outer surfaces before it can be transferred to the liquid helium cooling bath. Especially for abrupt disturbances, the absence of cooling passages creates near adiabatic operating conditions. The $g(t)$ term represents disturbance-induced heat generation. Because of its time- and temperature-dependent numerical complexity, a complete solution to Eq. 3.1 has yet to be evaluated. 
In 1972, Martinelli and Wipf ${ }^{2]}$ suggested the existence of a "disturbance spectrum" to characterize, in both time and space, disturbances capable of driving a superconducting magnet frem its metastable operating point. [3] The disturbance spectrum, depicted in Table 3.1, has since been used to guide the development of several approximate solutions to Eq. 3.1. ${ }^{[4]}$ Each solution corresponds to one of the extreme corners of Table 3.1 .

Table 3.1: Proposed Disturbance Energy Spectrum

\begin{tabular}{|c||cc|}
\hline \multirow{2}{*}{ Time Domain } & \multicolumn{2}{c|}{ Spatial Domain } \\
\cline { 2 - 3 } Continuous & Distributed & Local (e.g. point) \\
Transient & $\mathrm{W} / \mathrm{m}^{3}$ & $\mathrm{~W}$ \\
$\mathrm{~J} / \mathrm{m}^{3}$ & $\mathrm{~J}$ \\
\hline
\end{tabular}

\subsubsection{Continuous disturbances}

The most frequent source of continuous disturbances are steady electrical or electromagnetic dissipations within the winding. An example of a continuous point disturbance is a high resistance joint between two conductors. An example of a continuous distributed disturbances is the heat generated by changes in the superconducting filaments' magnetic flux densities. There are two primary causes of flux motion in the filaments. The first occurs when the overall flux density in a magnet is changing and is most frequently observed during magnet charging and discharge. The flux energy dissipated during electromagnetic cycling is known alternatively as magnetic hysteresis losses or ac losses depending on the cycling rate and frequency. ${ }^{[5]} \mathrm{A}$ second type of continuous distributed disturbance, known as flux creep, is observed near the conductor's critical current and is believed to result from slight irregularities in the filament properties along the conductor length. [6]

Although flux-creep-induced dissipation does not normally pose a problem to high-performance magnet stability, it does provide a rough measure of level of power dissipation compatible with stable magnet operation. Wilson ${ }^{[7]}$ has produced a rough analysis of this effect at $4.2 \mathrm{~K}$ for a superconducting winding consisting of $30 \% \mathrm{NbTi}, 40 \%$ copper, and $30 \%$ organic insulation. His analysis indicates that a 
6 - $\mathrm{T}$ solenoid operating at an overall critical current density, jave, of $\sim 3 \times 10^{8} \mathrm{~A} / \mathrm{m}^{2}$ generates $\sim 3.0 \mu \mathrm{W} / \mathrm{mm}^{3}$ due to flux creep induced dissipation. His analysis also suggests that transient heating rates as high as $20 \mu \mathrm{W} / \mathrm{mm}^{3}$ are permissible for the same magnet operating at $90 \%$ of its critical current.

If for some reason a magnet's operating current is raised to above its critical superconducting current, its power dissipation abruptly rises by about four orders of magnitude, as flux creep induced heating in the superconducting filaments (represented by an equivalent electrical resistivity of $\sim 10^{-14} \Omega \mathrm{m}$ ) gives way to ohmic dissipation in the copper matrix (with an equivalent resistivity of $\sim 10^{-10} \Omega \mathrm{m}$ ). The same effect occurs when the local conductor temperature rises above the critical superconducting temperature; the temperature excursion effectively reduces the conductor's critical superconducting current to below its fixed operating current. The rapid rise in ohmic heating that ensues produces a rapid growth in the size of the normal zone and lead to a thermal runaway that quenches the entire magnet.

The low level of power generation that can be tolerated in a high-performance magnet is generally not compatible with moderately high-power, continuous disturbances like ac losses or resistive joints. Because the magnitudes of these disturbances are usually very predictable, steps can be taken to accommodate them during the design process. The most frequently used approach is to provide limited cooling within the bulk of the winding, just sufficient to accommodate the steady-state dissipation; this help to maintain the economic advantages provided by a highcurrent-density design and yet allows for metastable operation in the presence of continuous power generation. ${ }^{[8,9]}$ However, since only partial cooling is provided it may be appropriate to classify these as quasi-adiabatic high-performance magnets because they are still susceptible to quenches resulting from transient thermal disturbances. ${ }^{[8]}$

\subsubsection{Transient disturbances}

\subsubsection{Distributed disturbances}

Transient disturbances occur so rapidly that virtually all of their energy is converted to temperature rise, localized about the disturbance event. The transition from this local temperature rise to quenching occurs on a time scale far shorter than 
it takes for the disturbance energy to be conducted to the winding's boundaries. Under these conditions, the magnet's quench energy density, $e_{q}$, can be approximated by the change in enthalpy needed to raise the magnet from its operating temperature, $T_{o p}$, to its critical superconducting temperature, $T_{\mathrm{c}}$. For a 'typical winding: consisting of $30 \% \mathrm{NbTi}, 40 \%$ copper and $30 \%$ organic insulation, this quench energy density is $\sim 3.0 \mu \mathrm{J} / \mathrm{mm}^{3}$ for a temperature margin, $\left(T_{c}-T_{o p}\right)$, of $1.0 \mathrm{~K}$. [4]

A magnet's quench energy density is not a fixed quantity, rather, it varies with operating current and location within the winding. At low currents the magnet's temperature margin is high, and hence, so is its quench energy density. As the magnet nears its critical current the temperature margin decreases and the magnet becomes progressively less stable. The quench energy density is lowest at the point of peak flux density in the winding, $B_{w}$, as the magnet approaches its designed operating current. Figure 3.1 depicts the variation in quench energy density with operating current for the peak field region of a 'typical winding' operating at $4.2 \mathrm{~K}$. To accommodate potential changes in conductor dimensions between applications the operating current in this graph has been normalized with respect to the magnet's critical superconductor current.

\subsubsection{Point disturbances - minimum propagating zones}

The available experimental evidence, to be reviewed in $\$ 3.2 .2$, suggests that abrupt disturbances, particularly those localized to regions smaller in cross section than a single conductor diameter, are the dominant source of premature quench in contemporary high-performance magnets. The most frequently cited analysis of these "point" source disturbances is the minimum propagating zone, MPZ, theory originally suggested by Martinelli and Wipf., ${ }^{[2]}$ and later expanded upon by Wilson. ${ }^{4]}$ According to the MPZ concept it is theoretically possible for a small, critically-sized normal region to exist in thermal equilibrium with an otherwise superconducting winding, as long as the resistive energy dissipated in this normal zone is balanced by heat conduction through the bulk of the winding to the helium coolant bath. A normal zone smaller than this critical size would recover to the superconducting state, whereas slightly larger zones will grow in size, that is, propagate $^{[2]}$. The critical dimensions of this MPZ can be derived as a special case 


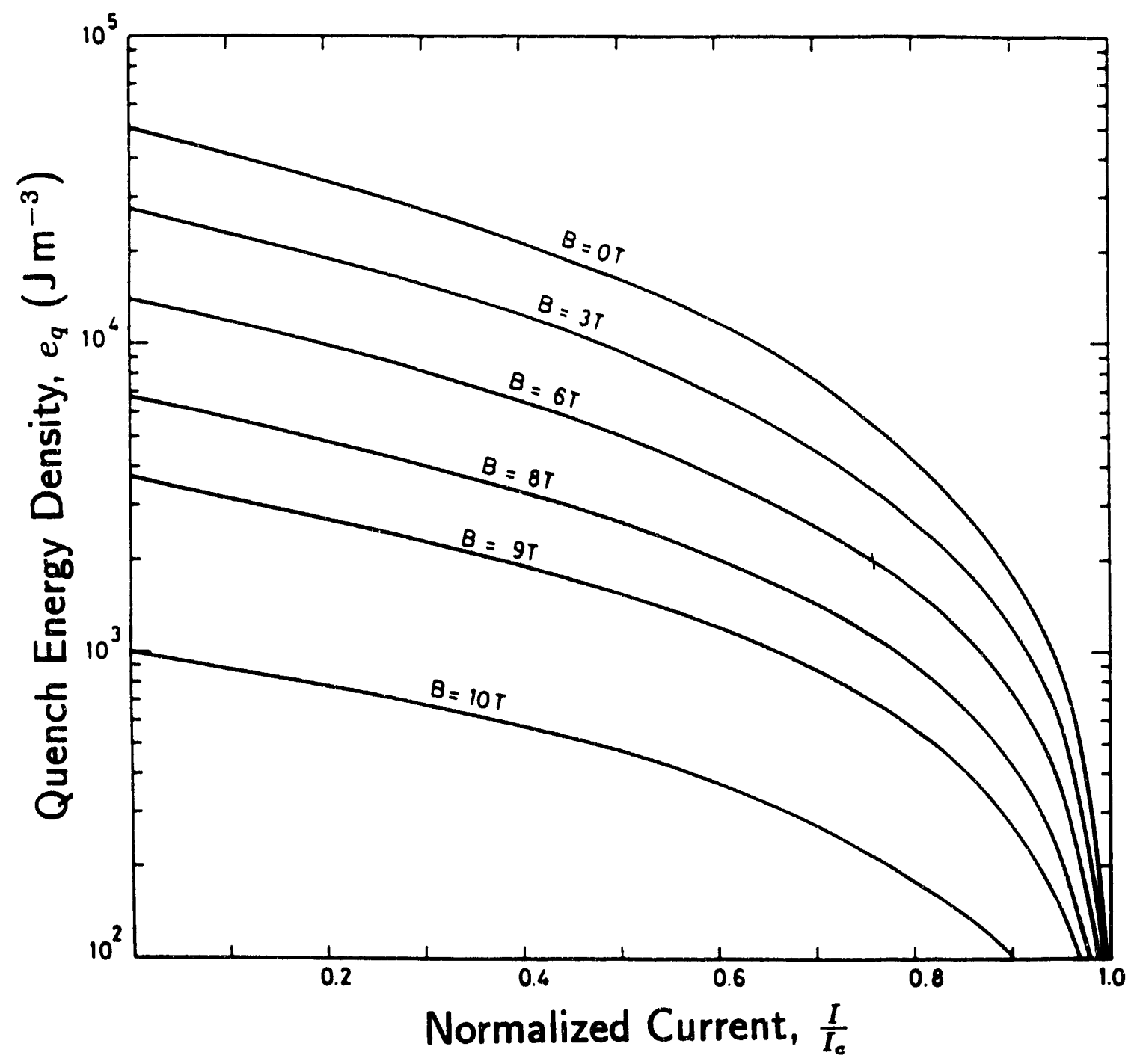

Figure 3.1: Parameterized curves of quench energy density vs normalized operating current, at various fixed magnetic flux densities, for a 'typical' $\mathrm{NbTi}$ winding at $4.2 \mathrm{~K}$. Figure from [4]. 
of Eq. 3.1 subject to the conditions: $d T / d t=0 ; g(t)=0$; and $h(T)=0 \cdot \cdot^{[3,4]}$

$$
\nabla \cdot\left(\left[k_{w}\right] \nabla T\right)+\rho_{w}(T) j_{a v e}^{2}=0
$$

In evaluating this equation, it should be born in mind that the winding's thermal conductivity along the conductor axis, $k_{z}$, is much larger than its transverse conductivity, $k_{t}$. The winding's anisotropic thermal conductivity thus results in a highly ellipsoidal MPZ. The major radius of this ellipsoid, $R_{z}$ extends along the conductor axis and is given by: ${ }^{[4]}$

$$
R_{z} \simeq \pi \sqrt{\frac{k_{z} \lambda_{c u}\left(T_{c}-T_{o p}\right)}{\rho_{c u} j_{a v e}^{2}}}
$$

If $\kappa^{2}=k_{t} / k_{z}$ is used to represent the thermal anisotropy of the winding, the length of the minor radius, $R_{t}$, is $\kappa R_{z}$. The right hand side of Eq. 3.3 relates the average power generation inside the resistive normal zone, $j_{a v e}^{2} \rho_{c u} / \lambda_{c u}$ to the heat conducted out of the normal zone, $k_{z}\left(T_{c}-T_{o p}\right)$. The matrix resistivity, $\rho_{c u}$, is used in Eq. 3.3 because, in the normal state, virtually all of the power dissipation takes place in the lower resistivity copper matrix.

Substituting in approximate values for a 'typical' $6 \mathrm{~T}$ magnet, namely $j_{a v e}=3 \times 10^{8} \mathrm{~A} / \mathrm{m}^{2},\left(T_{c}-T_{o p}\right)=1 \mathrm{~K}, \rho_{c u}=3 \times 10-10 \Omega \mathrm{m}, k_{z}=350 \mathrm{~W} / \mathrm{m}-\mathrm{K}$, and $k_{t}=1.0 \mathrm{~W} / \mathrm{m}-\mathrm{K}$, yields $R_{z}=7 \mathrm{~mm}$ and $R_{t}=0.4 \mathrm{~mm}$. For an abrupt point source disturbance to initiate a quench, it must dissipate sufficient energy to raise the entire MPZ volume from its operating temperature to just above its critical superconducting temperature. Based on the parameters for the $6 \mathrm{~T}$ magnet used in this example, and a $1 \mathrm{~K}$ temperature margin, the MPZ theory predicts a minimum quench energy, $\varepsilon_{\min }$, of $\sim 15 \mu \mathrm{J}$.

$$
\varepsilon_{\min }=e_{q} \frac{4 \pi}{3} R_{t}^{2} R_{z}
$$

The general conclusions of the MPZ theory have been experimentally verified by Schmidt, ${ }^{[10]}$ Superczynski, ${ }^{[1]}$ and Scott, ${ }^{[12]}$ who demonstrate that energy pulses as small as $10 \sim 50 \mu \mathrm{J}$ are indeed sufficient to quench high-performance superconducting windings. Their experimental results likewise indicate that as the time scale of 
the disturbance increases to above the winding's thermal diffusion time constant. the quantity of energy needed to quench the magnet markedly increases. Figure 3.2 shows the total pulse energy needed to quench a magnet for various disturbance pulse widths. ${ }^{[11]}$ The temperature rise for short pulses is essentially adiabatic, whereas thermal diffusion plays an increasing role in the thermal behavior as the pulse width increases.

\subsection{Characterization of transient point disturbances}

\subsubsection{Flux jumping}

Although the phenomena of superconductivity was first observed in elemental metals by Kammerling Onnes in 1911 it was not until the early to mid 1960's, with the discovery of high-field, high-current density superconductors like $\mathrm{NbZr}$ and $\mathrm{Nb}_{3} \mathrm{Sn}$, that superconducting magnets became a practical reality. ${ }^{[13]}$

The largest impediment to the development of early magnets resulted because of the manner in which the superconductors were used. ${ }^{[14]}$ These early magnets were wound from insulated wires consisting almost exclusively of pure superconductor. The ratio of the normal state resistivity to thermal conductivity for a pure superconductor is very low, approximately six and a half orders of magnitude less than that for subsequent copper-stabilized wires. Hence, Eq. 3.3 and Eq. 3.4 indicate that the disturbance energy needed to quench an early magnet is several orders of magnitude below that needed to quench a contemporary magnet. ${ }^{[4]}$

The second reason for the poor performance of early magnets is that the diameters of the pure superconducting wires were much larger than $100 \mu \mathrm{m}$. Subsequent analysis has shown that superconducting flaments above $\sim 100 \mu \mathrm{m}$ are increasingly prone to a spontaneous, electromagnetic-thermal instability known as flux jumping. ${ }^{[14]}$ During a flux jump, the motion of magnetic flux through the filament dissipates energy. The heat produced by this flux motion raises the local conductor temperature and lowers the conductor's local critical superconducting current density. This reduction in current density causes further flux motion, thus completing a flux-motion/heating feedback cycle that typically concludes with a magnet quench. ${ }^{[14]}$ The development of multifilamentary, composite conductors in the mid 1960 's provided a tractable solution to the problem of spontaneous flux jumping. 


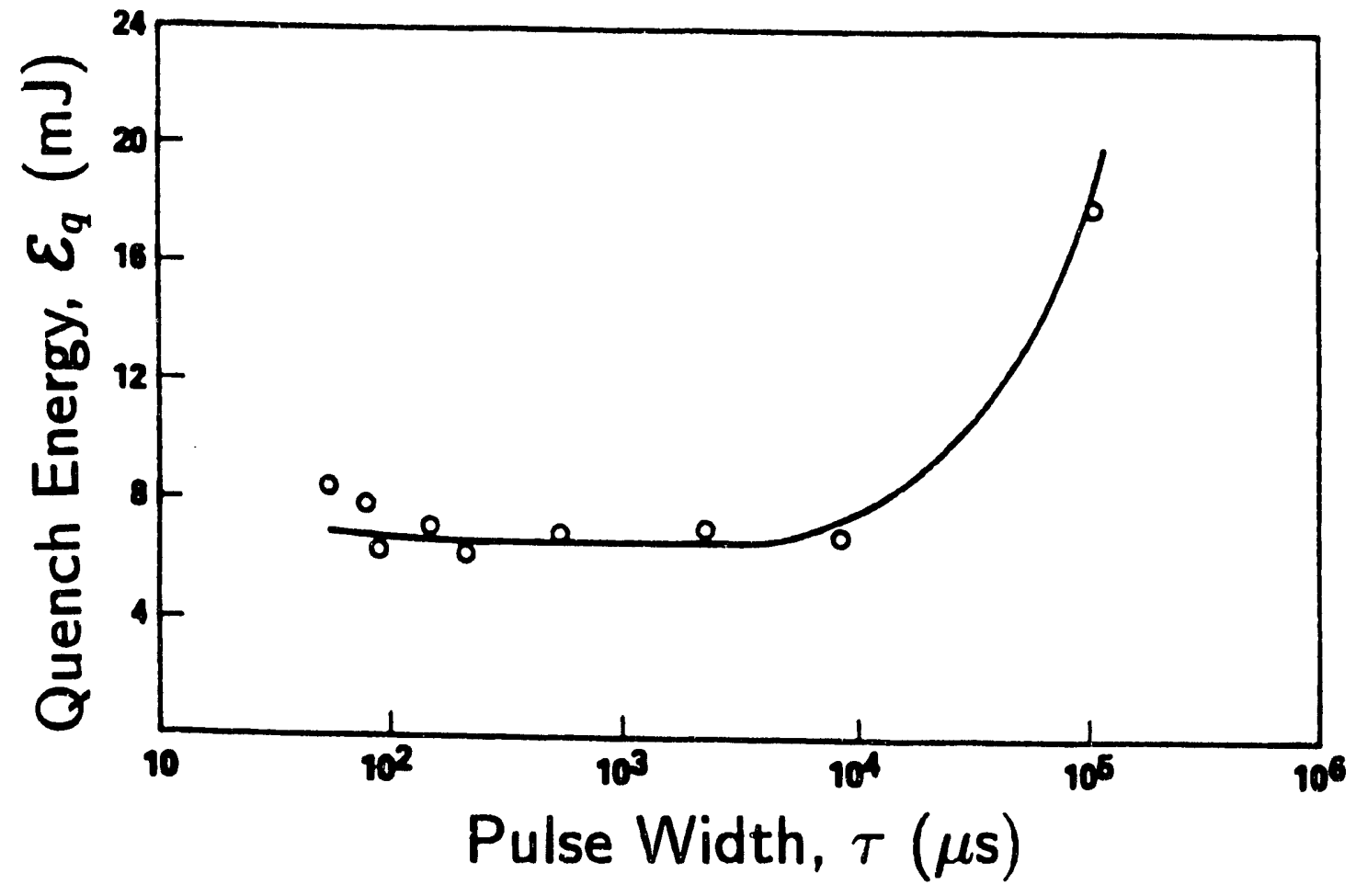

Figure 3.2: "Minimum" quench energy vs pulse width for an epoxy impregnated superconducting winding. Figure from [11]. 
The small filament diameters restrict flux-motion heating by limiting the total extent of spontaneous flux motion, while the high thermal conductivity of the copper matrix provides a much more uniform operating temperature.

Despite the tremendous improvements in magnet performance provided by the advent of multifilamentary conductors, the quench and training behaviors of highperformance magnets remains highly variable to the present day. The degradation that persists in contemporary magnets is commoniy attributed to small-scale. highly-localized mechanical disturbances..$^{(3,4,15-20]}$ The two most frequently cited disturbances sources are: epoxy fracture in potted magnets and frictional motion in dry wound coils. A third major type of quench-initiating mechanical disturbance, namely, microplastic yielding of the superconducting filaments, has also been identified. Fortunately, microplastic yielding in NbTi conductors can be prevented by applying sufficient cold work during manufacture to ensure that the conductor remains entirely in the elastic regime during magnet operation. ${ }^{[21-23]}$

\subsubsection{Mechanical disturbances}

\subsubsection{Mechanical stresses}

The mechanical stress state in a superconducting magnet can be divided into three components. ${ }^{[24,25]}$ The first arises from the conductor tensions used during the winding process. The second, from differential thermal contractions between the winding constituents as the magnet is cooled to its 4.2 - $\mathrm{K}$ operating temperature. The third component results from electromagnetic, or Lorentz, stresses which are produced when the magnet is energized.

The initial stress state in a magnet is determined entirely by the conductor tension during manufacture and by thermal contraction. As the magnet is charged towards its $4.2-\mathrm{K}$ operating point, its stress state changes. The changing operating current during energization produces a corresponding increase in the winding's distributed electromagnetic body forces, $\overline{\mathbf{J}} \times \overline{\mathbf{B}}$ per unit volume. In a high-performance magnet where both the field and current density are high the electromagnetic body forces can be quite large and generate appreciable stress. For the most part, these body forces are accommodated by elastic deformation of the magnet. However, due to the presence of nonuniform stresses and unintended stress concentrations 
these electromagnetic forces can also trigger abrupt, highly-localized mechanical disturbances.

All real solenoids have finite length, hence, both their magnetic inductions and attendant electromagnetic body forces vary throughout the winding. Figure 3.3a shows a schematic mapping of lines of constant magnetic induction for a solenoid. while Figure $3.3 \mathrm{~b}$ depicts the per-unit-volume electromagnetic force vectors. ${ }^{[4]}$ The length and angle of these arrows represents the magnitude and direction of the electromagnetic body forces. A quantitative analysis of these forces will be developed in Chapter 7; for the present discussion, a qualitative description will be sufficient.

A solenoid's magnetic induction, $\overline{\mathrm{B}}$, consist of two components. The principal component is the axial induction, $B_{z}$. The axial induction is greatest at the coil's midplane and decreases markedly due to fringing effects near the coil ends. The radial induction, $\mathrm{B}_{r}$, is negligible near the coil midplane but increases due to the fringing fields near the coil's ends to as much as $50 \%$ of the magnet's central induction. The cross-product between the circumferentially directed current density and the axial magnetic induction produced an outward-directed radial electromagnetic force component, $f_{r}^{\prime}=j_{a v e} \times B_{z}$. Similarly, the radial induction produces an axial force per unit length, $f_{z}=j_{\text {ave }} \times B_{r}$, directed towards the coil's midplane.

\subsubsection{Disturbance characterization}

Several types of disturbances have been observed in high-performance magnets. Most of these have been qualitatively interpreted in terms of the axial and radial electromagnetic force components. By far the largest proportion of research has been conducted on epoxy-impregnated windings. However, the recent development of successful methods for minimizing quenching in potted magnets has since led to renewed interest in the performance capabilities of dry-wound magnets. The four most common mechanical sources of quenching in high-performance solenoids are listed below.

1. Winding/coil-form debonding can occur in an impregnated magnet whose winding is adhered to coil form. The radial electromagnetic force component produces a radial, outward expansion of the winding. If the radial tensile stress that develops at the winding-coil form interface exceeds the epoxy's tensile 


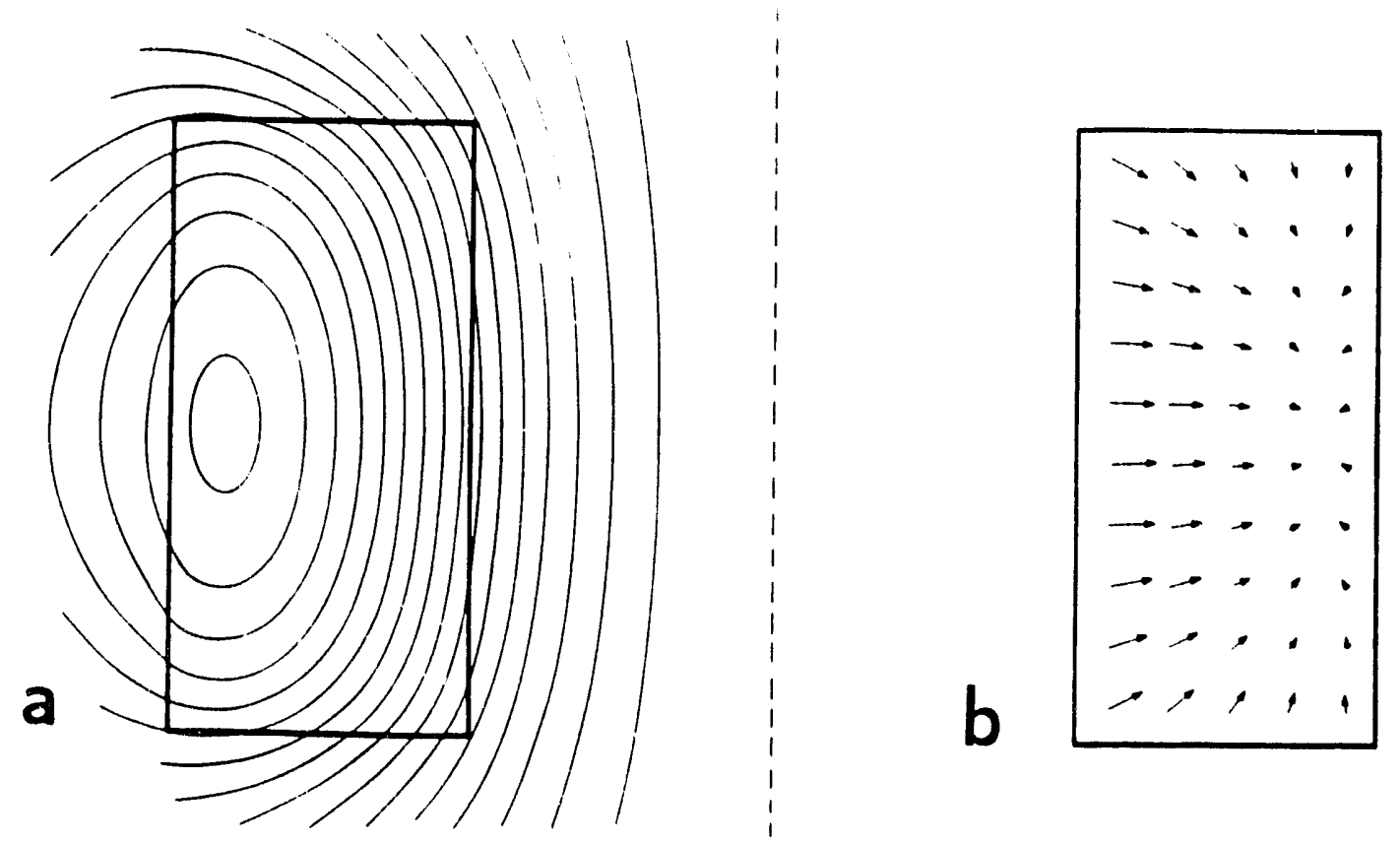

Figure 3.3: a) Parameterized contours of constant magnetic induction. b) Electromagnetic force vectors. The dotted line represents the winding centerline. Figure from $[4]$. 
strength, debonding occurs. The fracture energy associated with debonding is frequently capable of quench initiation. One method developed to prevent debonding is to use the conductor tension during manufacturing to create a large enough hoop stress to counteract this tendency towards radial separation. ${ }^{\text {[26] }}$ This method does not extend well to large-bore, high-field magnets where the electromagnetic stresses already constitute a large fraction of the conductor's tensile strength.

2. Shear-stress-induced epoxy cracking can also occur in impregnated magnets when the winding is firmly bonded to the coil form. The axial restraint imposed on the winding by the bore tube can produce large shear stresses, particularly in the inner winding layers near the coil's ends. ${ }^{[27-29]}$ Because the interconductor shear stresses are transmitted solely by the epoxy impregnant, fracture can occur at relatively modest overall stress levels. One of the methods used to combat this disturbance source is the use of very-low-shear-strength impregnants such as wax; this method derives from the premise that quench can be prevented if the strain energy dissipated at fracture is kept very low. ${ }^{[30]}$ A second method for preventing shear-stress-fracture-induced quenches is the "floating coil" design. $[3,25,27,28]$ In this method the winding is allowed to freely separate from its coil form during energization. By allowing the coil to seek its natural shape, the shear stresses are substantially reduced. This floating coil concept has since made epoxy-impregnated solenoids virtually immune to premature quenches. ${ }^{[3]}$ The floating coil concept has since been further extended to include coil-formless solenoids in which the coil former is entirely removed form the winding before operation. ${ }^{[32,33]}$

3. Global, or interfacial, winding motion can occur in an impregnated winding that is not adhered, but mechanically pressed against its support structure. ${ }^{[20]}$ A quench can occur if sufficient frictional heating is transferred from the interface to the conductors. Interfacial-motion-induced quench's have been observed in dipole and solenoidal windings. ${ }^{[34,35]}$ In these windings, the distributed axial electromagnetic body force produces an axial friction force along the support structure interface. Motion occurs when the winding's net interfacial friction force exceeds its static friction force. Because the electromagnetic 
body force varies in a regular manner as the coil is energized, quenches resulting from this type of motion are often repeatable and can prevent the winding from ever reaching its design performance.

Two methods have been used to minimize interfacial-motion-induced quenches in solenoidal magnets. Because frictional heating can only occur when these is contact between the winding and coil form, interfacial motions in impregnated solenoids can typically be prevented by inducing the coil to radially separate from its coil form while its charging current is still low in comparison to the critical current. ${ }^{[31]}$ In this case, frictional heating is prevented by eliminating radial contact between the surfaces. Low-thermal-conductivity friction blocks can also be mounted to a coil's boundaries to help decouple the frictional heating source from the winding; by increasing the conduction path to the winding proportionally more of the frictional heat is transferred to the magnet's cooling bath, resulting in a much lower peak temperature in the winding. ${ }^{[31,36]}$ No methods have yet been advanced to alleviate interface-motion-induced quenches in dipole magnets.

4. Local, or individual, conductor motions were the earliest identified source of mechanical disturbance in high-performance magnets, ${ }^{[15]}$ and yet the latest to be extensively investigated. ${ }^{[37-40]}$ In the interim, considerable research effort has been devoted to completely eliminate the possibility of individual conductor motions through the use of suitable organic impregnants that are used to transform the winding into a single structural unit. With the development of floating and coil-formless windings, impregnated solenoidal windings have become extremely reliable. There are, however, major drawbacks to the use of impregnated windings. One is their relatively large manufacturing cost compared to unimpregnated windings. Several processing steps are needed to: a) introduce the impregnant into the windings, b) ensure uniform impregnant properties, c) avoid air bubbles of other defects, and d) prevent adhesion to the coil former. A second is its irrevocability; once a magnet has been impregnated it is difficult, but not impossible, to recover the conductor. Hence, from an economic standpoint, there has been a renewed manufacturing interest in investigating methods for improving dry-wound solenoid reliability. 


\subsection{Mechanical disturbance detection}

Principally three diagnostic tools have been developed to monitor mechanical disturbances in high-performance magnets. They are: 1) strain gauges, ${ }^{(37,39 !}$ 2) acoustic emission ( $\mathrm{AE}$ ) transducers, ${ }^{\{3,19,41,42\}}$ and 3) differential voltage taps. ${ }^{[18,43,44]}$

\subsubsection{Strain gauges}

Strain gauges have been used to measure the changes that occur in the axial and circumferential strains at the outer surfaces of magnet windings during energization. ${ }^{[34]}$ These strain measurements are primarily used to verify the accuracy of analytic models developed to predict the solenoids' mechanical stress-strain behaviors. Gauges mounted to the inside of the bore tube have likewise been used to detect the occurrence of radial separation between the winding and coil form. ${ }^{[37]}$ As the winding separates the strain in the coil form becomes constant. Strain gauges have recently been used to measure the extent of axial motion in single-laver, unimpregnated winding models. ${ }^{[39]}$

\subsubsection{Acoustic emissions monitoring}

All abrupt mechanical disturbances produce a rapid change in the local stress state in the winding. ${ }^{[3]}$ This change in the stress state produces an acoustic wave that propagates outward through the winding. Thus, the occurrence of mechanical disturbances can be inferred using acoustic emissions (AE) transducers closely coupled to the winding to detect these acoustic signals.

The widespread use of $\mathrm{AE}$ technology for monitoring superconducting magnets first began in the late 1970 s. $^{[17,41]}$ Since that time AE monitoring has been used to detect and identify the magnets' chief sources of mechanical disturbances. One of the more important terms that the magnet community has adopted from AEmonitoring terminology is the concept of a "Kaiser effect". ${ }^{[3,29]}$ The "Kaiser effect" describes the mechanical behavior observed during a period of cyclical loading in which disturbances occur only when the mechanical load responsible for the disturbance exceeds the maximum level achieved during the previous loading cycle.

Figure 3.4 shows an example of the Kaiser effect observed during microscopic 


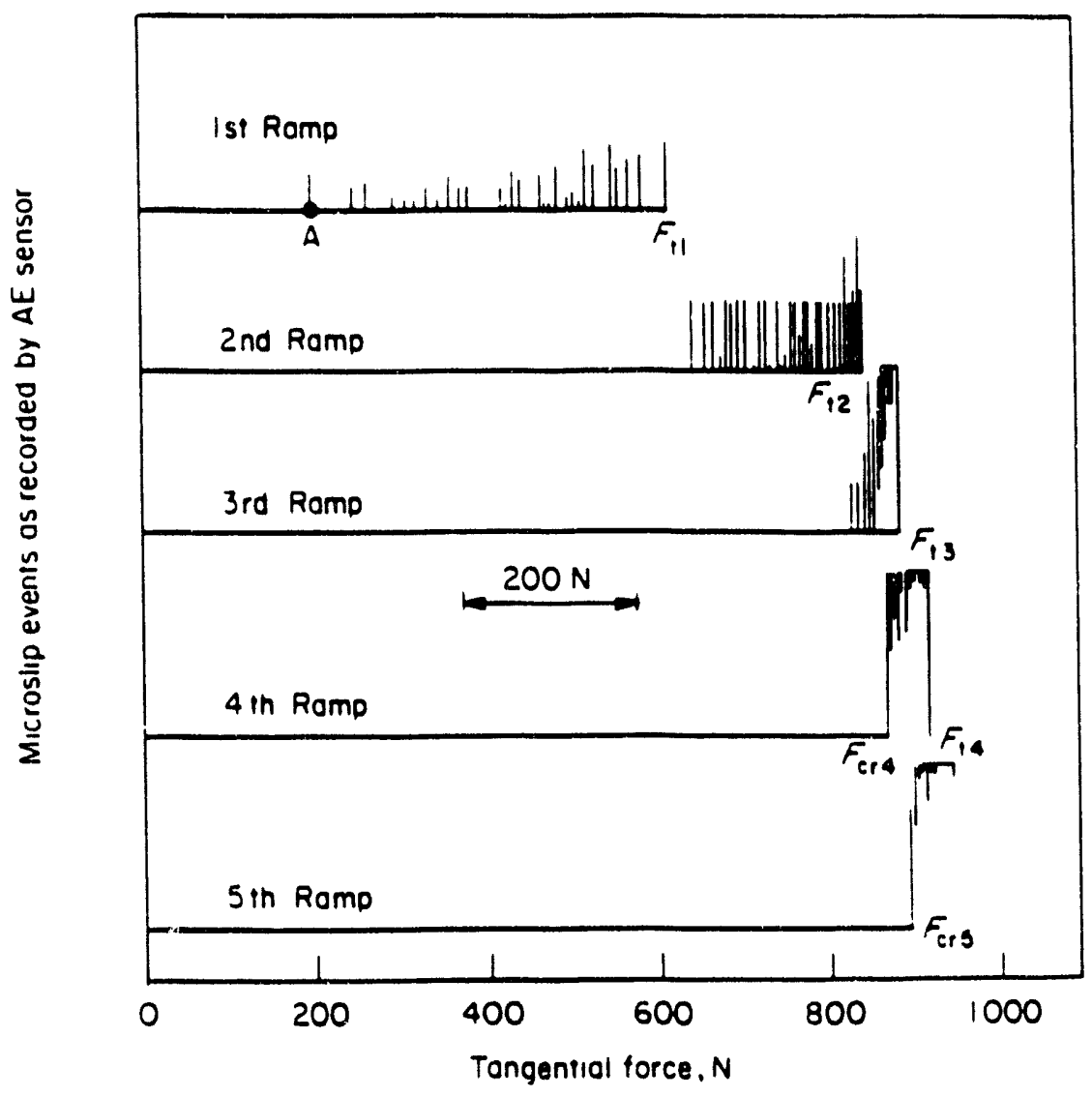

Figure 3.4: Example of the Kaiser effect observed during friction experiment between OFHC copper and G-10. Each vertical bar represents a microscopic slip event; the heigth of the bar corresponds to the conductor displacement. Figure from [45]. 
sliding experiments between oxygen-free-high-conductivity copper and a glass-fabric reinforced epoxy (G-10) composite, at a fixed normal force. ${ }^{[45]}$ The horizontal scale in these traces corresponds to the tangentially applied friction force, while the vertical axis shows the acoustic emission signals that were recorded during the test. Each AE event corresponds to a microscopic slip between the surfaces of a $1 \sim 10 \mu \mathrm{m}$ displacement. During the first loading sequence (top trace) the point A represents the friction force at which the first microslip was observed. Microslips continues until $F_{t 1}$, the maximum force during the first loading cycle. No microslips were observed during unloading. For the second loading cycle, microslips were observed only when the friction force increased above $F_{t 1}$ and continued until the loading sequence ended at $F_{t 2}$. Epoxy fracture disturbances generally follow a Kaiser effect behavior, leading to progressive training, whereas conductor motion disturbances may or may not.

\subsubsection{Differential voltage taps}

Another method for monitoring a magnet's performance during energization is to divide the magnet winding with voltage taps into several subsections. The differential voltages across these subsections can then be used to infer the occurrence of various events within that section. These voltage signals consist of up to three components. The first component, $V_{1}$, is the section's induced charging voltage, $V_{1}=L d I / d t$. When a slow, steady charging rate is used this component assumes a constant value. The second component, $V_{2}$, is the rapidly rising resistive voltage that follows quench initiation, $V_{2}=R(t) I(t)$. The resistive component is often used during quench propagation experiments to estimate both the size and temperature of the evolving normal zone. The third component, $V_{3}$, is an inductive voltage spike produced by a localized conductor motion within the winding. $V_{3}=d \Phi / d t$, where $\Phi$ is the flux swept out by the moving conductor. The shape of this voltage spike can be used to estimate both the spatial and temporal extent of motion.

Figure 3.5 presents a model for predicting the induced voltage during a conductor motion. ${ }^{[19]}$ The model assumes that the direction of motion, the conductor axis, and the local magnetic induction are all mutually perpendicular. If the center of a conductor segment of lergth $2 \ell$ is displaced laterally by a distance $\delta$ through a locally uniform magnetic induction, $B$, the segment's incluced voltage, $V_{3}$, is ap- 


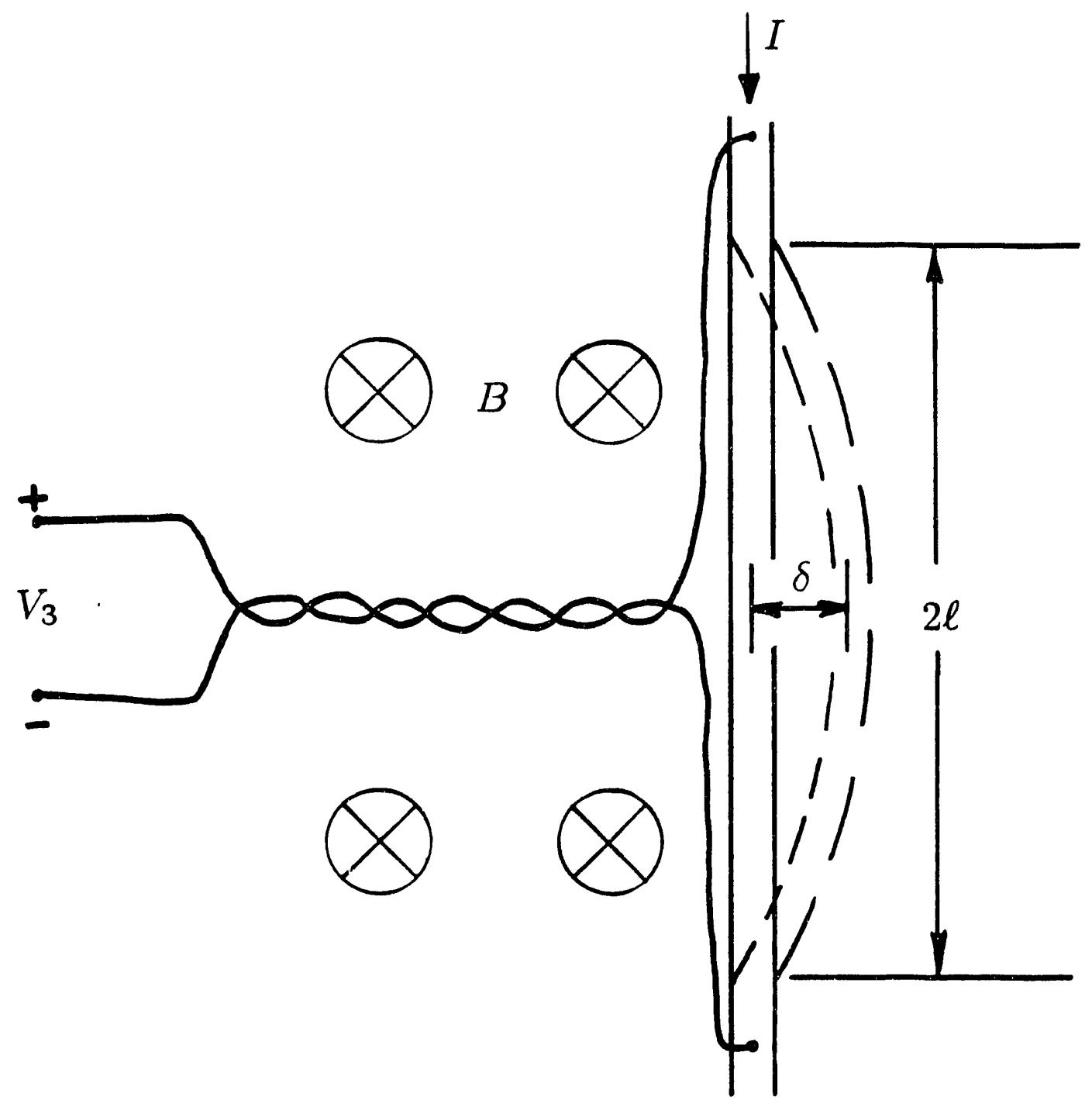

Figure 3.5: Simple model to predict the characteristics of the voltage transient produced by conductor motion in background magnetic induction [19] 
proximately:

$$
V_{3}=B \ell \frac{d \delta}{d t}
$$

Conversely, the total distance moved during conductor motion, $\delta$, can be estimated by integrating the induced voltage spike

$$
\delta=\frac{1}{B \ell} \int V_{3} d t .
$$

This conductor motion model has been used to experimentally determine the slip conditions capable of quench initiation. ${ }^{[19,43]}$ The experiments typically employed a single composite superconductor positioned in a uniform, externally applied magnetic field. The current in the conductor was then increased. Closely spaceci voltage taps ( $\sim 10 \mathrm{~mm}$ separation) were used during these tests to improve the spatial resolution of the voltage monitoring technique. AE monitoring was also used to help verify the mechanical origins of the voltage signals. Juitially, no signals were observed, however, as the current increased above a certain level AE pulses and voltage spikes began to occur concurrently. These signals continued sporadically until ultimately a quench occurred. For the configurations used, the experimental results suggest typical values for the slip duration of $0.1 \sim 1 \mathrm{~ms}$, moving segment lengths, $2 \ell$, of $10 \sim 30 \mathrm{~mm}$, and conductor displacements, $\delta$, in the range $1 \sim 10 \mu \mathrm{m}$.

Figure 3.6 presents an example of the $\mathrm{AE}$ and voltage signals obtained from the small-scale winding model tested in Chapter 7 . The results confirm that as the winding nears its critical current, the energy dissipated during a $5 \sim 10 \mu \mathrm{m}$ displacement is sufficient to induce a premature quench.

The measured conductor displacements can likewise be used to estimate a winding's approximate quench energy density. These estimates are generally derived based on electromagnetic force considerations. ${ }^{[14]}$ For a moving conductor segment the electromagnetic energy expended during the conductor motion, $\mathcal{E}$, is given by:

$$
\varepsilon=(I \ell \times B) \cdot \delta .
$$

A portion, $p$, of this energy is dissipated as frictional heating, while the remainder is stored as elastic strain energy in the conductor. The conductor's quench energy, $\varepsilon_{q}$ 


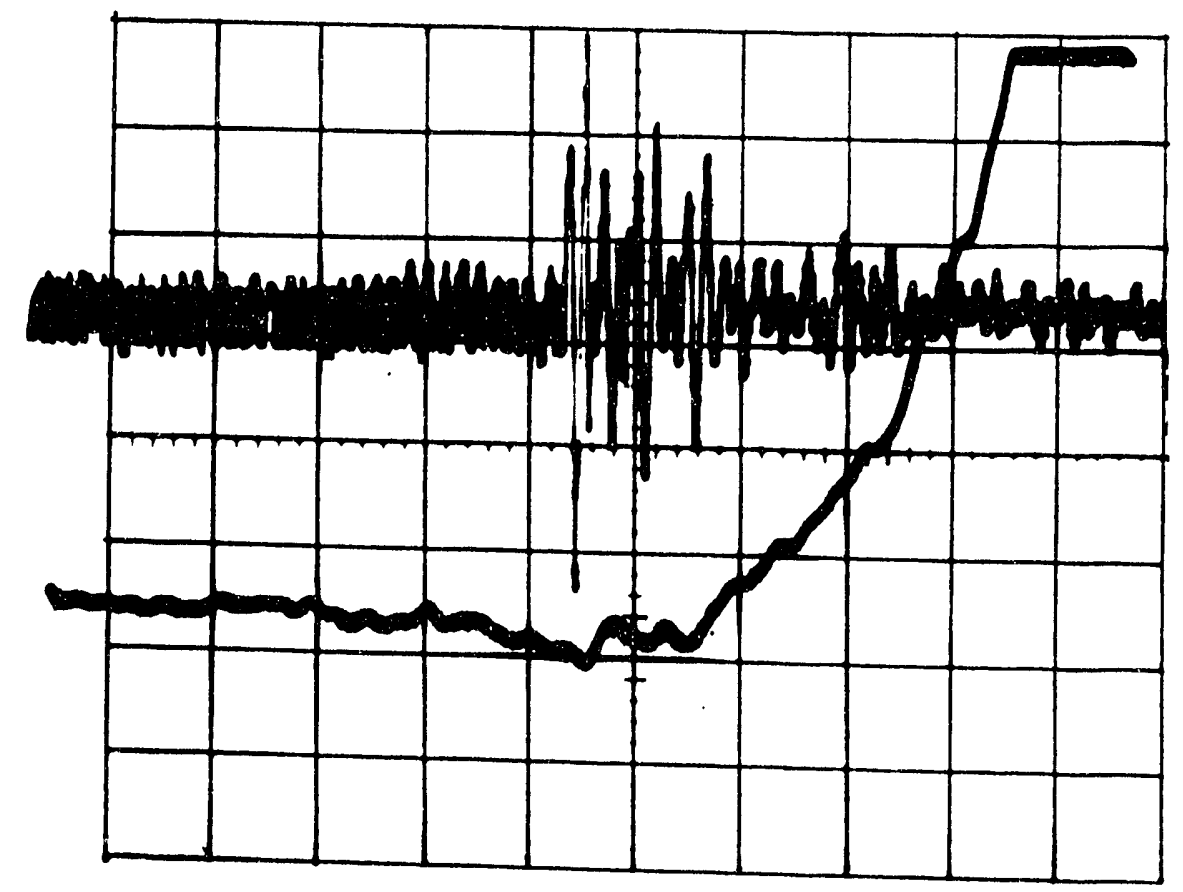

Figure 3.6: $\mathrm{AE}$ and voltage traces recorded at initiation of conductor motion induced quench. Top trace: AE transducer $\left(1 \mathrm{~V}_{f s}\right)$. Lower trace: Voltage tap signal (100 $\left.\mathrm{mV}_{f_{s}}\right)$. Time Base: $2.5 \mathrm{~ms}_{f_{s}}$. 
can be estimated by assuming that this frictional heat is transferred adiabatically only to the moving conductor segment.

$$
\varepsilon_{q}=p(I \ell \times B) \cdot \delta .
$$

In turn, the quench energy density, $e_{q}$, can be determine by dividing the conductor quench energy, $\mathcal{E}_{q}$, by the conductor segment volume. This gives:

$$
e_{q}=p\left(\frac{I \ell \times B}{A \ell}\right) \cdot \delta
$$

where $A$ is the conductor's cross-sectional area. Rewriting this equation and recognizing that $I / A$ is the winding's overall current density, $j_{a v e}$, gives:

$$
e_{q}=p j_{a v e} B \delta
$$

For a 6-T magnet with $p=0.5, j_{a v e}=300 \mathrm{~A} / \mathrm{mm}^{2}$, and $\delta=5 \mu \mathrm{m}$ the predicted quench energy density, $e_{\mathrm{q}}$, of $4.5 \mu \mathrm{J} / \mathrm{mm}^{3}$ compares quite favorably with the value of $3.0 \mu \mathrm{J} / \mathrm{mm}^{3}$ previously referenced in $\S 3.1 .3 .1$.

\subsubsection{Disturbance monitoring}

Perhaps the most useful technique yet developed for monitoring the operating performance of high-performance magnets is the combination of $\mathrm{AE}$ and voltage monitoring. For instance, abrupt mechanical disturbances can be readily detected by $\mathrm{AE}$ transducers mounted to both winding and coil form. In contrast to the small-scale, conductor-motion models discussed in the previous section, the voltage taps in an actual winding can generally be spaced no closer than about once per layer; this makes it almost impossible to determine the axial location of events in the winding. Additionally, both the axial and radial components of the magnetic induction vary markedly throughout the winding, hence, the extent of conductor motions are difficult to estimate since it is not possible to know for certain what value of $B$ or $\ell$ to use in evaluating Eq. 3.5. Despite these limitations, this combined $\mathrm{AE} /$ voltage monitoring technique has been used to identify three distinct types of quench. ${ }^{[19]}$ They are:

- Quench initiated by a voltage spike, accompanied by simultaneous AE burst.

- Quench accompanied by an AE burst but no voltage spike. 
- Quench with neither voltage spike nor AE signals.

All three types of quench are followed by a rising resistive voltage which can be used to indicate the radial location at which the quench occurred.

The following mechanism have been proposed for each type of quench.

(1) Quenches with a simultaneous AE burst and voltage spike are initiated by conductor motion. The voltage transient is produced by movement of an energized conductor within its surrounding magnetic field. Because a portion of the slip energy is released as an acoustic signal, the simultaneous occurrence of an $\mathrm{AE}$ burst with the voltage transient is evidence of the mechanical nature of the event. The radial location of the voltage taps where the spike occurs indicates the approximate position of the conductor motion.

(2) Quenches with AE bursts but no voltage transients are associated with internal disturbances which generated no discernible conductor movement. Examples of these disturbances include microplastic yielding of the superconducting filaments and shear-stress induced impregnant cracking.

(3) Quenches not accompanied by voltage spikes or AE are observed only when the conductor reaches its critical current. This type of quench is attributed to pure joule heating, which does not generate voltage spikes or AE signals.

\subsection{Summary}

The transient local heating generated during abrupt mechanical disturbances has been identified as the leading source of premature quench in high-performance superconducting solenoids. Several types of mechanical disturbances have been identified. They include: microplastic deformation of superconducting filaments; winding/coil-form debonding; shear-stress-induced impregnant cracking; winding/coil-form interfacial motion; and the microscopic slip of individual conductors within the winding. Construction methods have been devised to minimize the effects of all of these disturbances except localized conductor motions. Chapter 5 reviews the contemporary state of conductor-motion research. Recent advances in the development of cryogenic friction theory will be presented in Chapter 4 to provide a theoretical framework for discussing these conductor-motion studies. 


\section{References for Chapter 3}

1. Dresner, L. Superconductor stability, 1983: a review Cryogenics 24 (1984) 283292.

2. Martinelli, A.P. and Wipf, S.L. Investigation of cryogenic stability and reliability of operation of $\mathrm{Nb}_{3} \mathrm{Sn}$ coils in helium gas environment Proc. $1972 \mathrm{Appl}$. Supercond. Conf. IEEE Pub. 72ch0682-5-TABSC (1972) 331-340.

3. Iwasa, Y. Mechanical disturbances in superconducting magnets - A review IEEE Trans. MAG-28 (1992) 113-120.

4. Wilson, M.N. Superconducting Magnets Oxford University Press, New York (1983) pp 69-84.

5. Wilson, M.N. op. cit. pp 159-199.

6. Warnes, W.H., and Larbalestier, D.C. Critical current distributions in superconducting composites Cryogenics 26 (1986) 643-653.

7. Wilson, M.N. op. cit. pp 236-238.

8. Iwasa, Y. High-current-density magnets and its stability Cryogenics 31 (1991) 575-579.

9. Luguang, Y., Jie, Q., Changlian, Y., and Shiliang, W. A high current density superconducting solenoid with joints within the winding Proc. 11th Int. Conf. Mag. Tech. MT-11 Japan Institute of Electrical Engineers (1990) 1306-1311.

10. Schmidt, C. The induction of a propagating normal zone (quench) in a superconductor by local energy release Cryogenics 28 (1978) 605-610.

11. Superczynski, M.J. Heat pulses required to quench a potted superconducting magnet IEEE Trans. MAG-15 (1979) 325-327.

12. Scott, C.A. Minimum heat pulse to quench a superconducting magnet Stability of Superconductors International Institute of Refrigeration, Commission A 1/2 Saclay, France (1981) 189-194.

13. Wipf, S.L. Introduction to the discussion of stability - review of progress since the 1977 stability workshop Stability of Superconductors International Institute of Refrigeration, Commission A 1/2 Saclay, France (1981) 119-124.

14. Wilson, M.N., Walters, C.R., Lewin, J.D., and Smith, P.F. Filamentary superconducting composites - I. Basic ideas and theory J. Phys. D. Appl. Phys. 3 (1970) $1518-1546$.

15. Smith, P.F., Wilson, M.N., and Spurway, A.H. Filamentary superconducting composites - IV. DC coil tests J. Phys. D. Appl. Phys. 3 (1970) 1561-1573.

16. Evans, D. and Colyer, B.F. Sources of instability in superconducting magnets 
and possible explanations of the training problem Proc. 6th Int. Cryo. Eng. ('onf. IPC Science and Technology Press, Surrey, England (1976) 473-476.

17. Brechna, H. and Turowski, P. Training and degradation phenomena in superconducting magnets Proc. 6th Intl. Conf. Mag. Tech MT-6 ALFA, Bratislava, Czechoslovakia (1978) 597-611.

18. Tollestrup, A.V. Care and training of superconducting magnets IEEE Trans. MAG-17 (1981) 863-872.

19. Tsukamoto, O., Maguire, J.F., Bobrov, E.S., and Iwasa, Y. Identification of quench origins in a superconductor with acoustic emission and voltage measurements Appl. Phys. Lett. 39 (1981) 172-174.

20. Iwasa, Y. Experimental and theoretical investigation of mechanical disturbances in epoxy-impregnated superconducting coils. 1. General introduction Cryogenics 25 (1985) 304-306.

21. Kroeger, D.M., Easton, D.S., and Moazed, A. Stress-induced heating in commercial conductors and its possible influence on magnet performance IEEE Trans. MAG-13 (1977) 120-123.

22. Schmidt, C. and Turck, B. A cure against 'training' of superconducting magnets Cryogenics 17 (1977) 695-696.

23. Pasztor G. and Schmidt, C. Dynamic stress effects in technical superconductors and the "training" problem of superconducting magnets J. Appl. Phys. 49 (1978) 886-899.

24. Arp, V. Stresses in superconducting solenoids J. Appl. Phys. 48 (1977) 2026-2036.

25. Bobrov, E.S. and Williams, J.E.C. Stresses in superconducting solenoids Mechanics of Superconducting Structures, ASME Applied Mechanics Seminar AMD41 (1980) 13-41.

26. Bobrov, E.S., and Williams, J.E.C. Direct optimization of the winding process for superconducting solenoid magnets (Linear programming approach) IEEE Trans. MAG-17 (1981) 447-448.

27. E.S. Bobrov, J.E.C. Williams, and Y. Iwasa, Experimental and theoretical investigation of mechaniced disturbances in epoxy-impregnated superconducting coils. 2. Shear-stress-induced epoxy fracture as the principal source of premature quenches and training - theoretical analysis, Cryogenics 25 (1985) 307-316.

28. Y.Iwasa, E.S. Bobrov, O. Tsukamoto, T.Takaghi, and H. Fujita, Experimental and theoretical investigation of mechanical disturbances in epoxyimpregnated superconducting coils. 3. Fracture-induced premature quenches, Cryogenics 25 (1985) 317-322.

29. H.Fujita, T. Takaghi, and Y. Iwasa, Experimental and theoretical investiga- 
tion of mechanical disturbances in epoxy-impregnated superconducting coils. 4. Prequench cracks and frictional motion, Cryogenics 25 (1985) 323-326.

30. P.F. Smith and B. Colyer, A solution to the "training" problem in superconducting magnets, Cryogenics 15 (1975) 201-207.

31. Williams, J.E.C., Pourrahimi, S., Iwasa, Y.. and Neuringer, L.J. $600 \mathrm{MHz}$ spectrometer magnet IEEE Trans MAG-25 (1989) 1767-1770.

32. Maeda, H., Urata, M., Ogiwara, H., Miyake, S., Aoki, N., Sugimoto, M., and Tani, J. Stabilization for wind and react $\mathrm{Nb}_{3} \mathrm{Sn}$ high field insert coil Proc. 11th Inter. Conf. Mag. Tech. MT-11 Japan Institute of Electrical Engineers (1990) 1114-1119.

33. Urata, M., Maeda, H., Aoki, N., and Uchiyama, G. 17 Tesla magnet with $300 \mathrm{~mm}$ outer diameter IEEE Trans. MAG-27 (1991) 2391-2394.

34. Williams, J.E.C., and Bobrov, E.S. Magnet svstem of the $500 \mathrm{MHz}$ spectrometer at the Francis Bitter National Magnet Laboratory: II. Disturbances, quenches, and training Rev. Sci. Instr. 52 (1981) 657-661.

35. Ige, O.O. Mechanical disturbances in high-performance superconducting dipoles, PhD. Thesis, Dept. Mech. Eng., MIT (1989).

36. Iwasa, Y., Maguire, J.F., and Williams, J.E.C. The effect on stability of frictional decoupling for a composite superconductor Proc. 8th Sypm. Eng. Prob. of Fusion Res. IEEE Pub. 79CH1441-5 (1979) 1407-1411.

37. Urata, M., and Maeda, $\mathbf{H}$. Relation between radial stress and quench current for tightly wound dry solenoids IEEE Thans. MAG-23 (1987) 1596-1599.

38. Urata, M., and Maeda, H. Stabilization of superconducting dry solenoids IEEE Trans. MAG-25 (1989) 1528-1531.

39. Chikaba, J., Irie, F., Funaki, K., Takeo, M., and Yamafuji, K. Instabilities due to mechanical strain energy in superconducting magnets IEEE Trans. MAG23 (1987) 1600-1603.

40. Chikaba, J., Irie, F., Takeo, M., Funaki, K., and Yamafuji, K. Relation between instabilities and wire motion in superconducting magnets Cryogenics 30 (1990) 649-653.

41. Turowski, P. Acoustic emission and flux jump phenomena during training of superconducting magnets Proc. 6th Int. Conf. Mag. Tech. MT-6 ALFA, Bratislava, Czechoslovakia (1978) 648-653.

42. O. Tsukamoto, M.W. Sinclair, M.F. Steinhoff, and Y. Iwasa, Origins of acoustic emissions in superconducting wires, Appl. Phys. Lett. 38 (1981) 718-720.

43. Maeda, H. Mechanical disturbances for a cable-in-substructure superconductor Cryogenics 24 (1984) 208-210. 
44. Ogitsu, T., Tsuchiya, K., and Devred, A. Investigation of wire motion in superconducting magnets IEEE Trans. MAG-27 (1991) 2132-2135.

45. H. Maeda, O. Tsukamoto, and Y. Iwasa, The mechanisms of frictional motion and its effects at $4.2 \mathrm{~K}$ in superconducting magnet winding models, Cryogenics 22 (1982) $287-295$. 


\section{Cryogenic tribology}

In Chapter 4 the adhesive model of cryogenic-temperature polymer-on-metal sliding friction is developed. The classical, asperity junction model for adhesive friction is introduced and experimental evidence is presented to support the extension of this model to cryogenic temperatures.

Several investigations have previously been performed to characterize the frictional behavior of cryogenic-temperature sliding materials. ${ }^{[1-6]}$ These studies have been largely phenomenological in nature and have not typically suggested a physical mechanism for interpreting low-temperature friction phenomena. Separate tests have also been conducted to investigate frictional phenomena in superconducting magnets. These superconducting-magnet friction studies will be reviewed separately in Chapter 5.

\subsection{Asperity junction model for adhesive friction and wear}

Most friction and wear theories are based on the premise that the total area of contact between sliding materials is much smaller than their apparent surface areas. No engineering surfaces is perfectly smooth, consequently, the contact between solids is concentrated into a finite number of spots where the asperities, or high points, on the surfaces meet. As a normal load is applied to the interface, these asperity contacts deform to achieve a total contact area that is determined by the flow stress of the softer surface. For engineering materials this flow stress is usually equated with the materials' indentation hardness. However, for highly elastic materials such as rubbers, or materials which possess marked time- or temperaturedependent mechanical properties, it is not unusual for alternative measures of the material's flow properties to be used; those which more accurately reflect the material's actual deformation behavior.

The adhesion theory of friction and wear was originally formulated to interpret the sliding behavior of ideal, plastic materials. As such, it provides an appropri- 
ate description for the sliding behavior of structural materials like metals and rigid polymers. For these materials the total area of contact. $d_{r}$, can be estimated for a given load, $L$, based on the similarity between the assumed asperity contact conditions and the macroscopic conditions which prevail during an indentation hardness measurement.

$$
A_{r}=\frac{L}{P}
$$

where $P$ is the penetration hardness of the softer surface.

In many instances it is important to determine not only the total area, but also the number and average size of the asperity contacts. Several methods have been devised for this purpose. One is direct observation: in this method, one sliding surfaces is transparent so that the contacts can be directly measured. ${ }^{7]}$ Statistical estimates have also been tried. Autocorrelation analyses of friction traces have been used to estimate the average asperity contact diameter, based on the assumption that each asperity contributes a different. constant amount to the friction force for the life of the contact; as sliding continues the total friction force fluctuates as the asperity population changes. ${ }^{[8]}$ Statistical summaries of surface topographies have likewise been used in conjunction with the surfaces' mechanical properties and the normal load to estimate typical contact area distributions. [9] Despite the wide variety of approaches employed, all of these methods indicate typical junction size of $10 \sim 100 \mu \mathrm{m}$ for most engineering materials, and that total number of contacts, rather than their size, changes with load. This contact characterization is usually applicable so long as the total real area of contact remains a small fraction of the apparent area.

\subsubsection{Friction coefficients}

Polymer friction is attributed to two principal causes. ${ }^{[10]}$ One is adhesion, the intermolecular bonding that occurs in the asperity junctions that constitute the real area of contact between sliding materials. For sliding to occur the friction force, $F_{f}$, must exceed the junctions' collective shear strengths.

$$
F_{f} \geq s \cdot A_{r}
$$

where $s$ is the interfacial junction shear strength. Assuming that shear occurs along the original contact area, the adhesive friction coefficient, $\mu_{a}=F_{f} / L$, is expressed 
as the ratio of plastic strength parameters:

$$
\mu_{a}=\frac{a}{P}
$$

According to King and Tabor ${ }^{i 1\}}$ the interfacial shear strength, $s$, is often markedly different from the polymer's bulk shear strength. $S$, because the interfacial shear is affected not only by the intrinsic adhesion of the polymer's molecular chains to the opposing counterface but also by the high-hydrostatic pressures present at the sliding interface. By introducing a correction factor, $\mathrm{c}=s / S$, they were able to reconcile the temperature-dependent bulk mechanical properties of a number of polymers with their friction coefficients in the range $230-300 \mathrm{~K}$.

A special case of adhesive friction arises when a thin, low-shear-strength film is introduced into the interface. Under these boundary-lubricated conditions the friction coefficient, $\mu_{l}$, becomes:

$$
\mu_{l}=\alpha \frac{s_{l}}{P}+(1-\alpha) \frac{s}{P}
$$

where $\alpha$ is the fractional area of adhesive junctions covered by lubricant, and $s_{l}$ is the lubricant's shear strength. The friction coefficient of a sliding pair can be reduced considerably by completely separating the sliding surfaces $(\alpha=1)$ with a low shear strength lubricant $\left(s_{l} \ll s\right)$.

A second contribution to the friction coefficient is observed when the asperities, or high spots, on one surface penetrate into the opposing surface to an appreciable depth. For relative motion to occur, the material in front of the penetrated asperities must be physically displaced, resulting in the abrasion, or plowing, of the softer surface. Abrasion can also occur when hard, loose particles become trapped between the surfaces. If the penetrated abrasive is conical, with an roughness angle of $\Theta$, the plowing friction coefficient, $\mu_{p}$, is given by: ${ }^{[12]}$

$$
\mu_{p}=\frac{\tan \Theta}{\pi}
$$

Abrasion is undesirable in most applications because of its characteristically high friction and wear; fortunately, it is the one form of friction which can be virtually eliminated in practice by smoothing the bearing surfaces on harder materials, and by excluding abrasive particles from the sliding interface. 


\subsubsection{Wear coefficients}

It is occasionally observed that the interface formed during sliding is stronger than the surrounding material and that junction separation occurs along a path inside the asperity, rather than along the original interface. When this occurs a particle of material is transferred to the opposing surface. Additionally, if the elastic energy stored in the particle during its formation is large enough, the particle can

spring free of both surfaces leading to the formation of loose wear debris. ${ }^{[12]}$ This idealized representation of particle formation forms the basis of the adhesive theory of wear.

The most frequently cited formulation of the adhesive wear theory was presented by Archard and is summarized in the equation: ${ }^{[13]}$

$$
V=K \cdot \frac{L X}{P}
$$

Under steady-state conditions, a material's wear volume, $V$, is directly proportional to both the normal load, $L$, and distance slid, $K$, and inversely proportional to the hardness of the worn material. The parameter $K$ is a nondimensional coefficient that indicates the probability of forming an adhesive wear particle for each asperity encounter. Subsequent experimental research indicates that this relation closely simulates the the observed relation between the major wear parameters not only for adhesive wear, but also for abrasion, the other principal form of wear. ${ }^{[14]}$ Even though the form of the Archard equation remains constant, the $K$ values for each wear mechanism are often vastly different. Hence, the magnitude of $K$ can often be used to identify the wear mechanism for a given set of experimental results. The adhesive wear coefficients for polymers are usually in the range $10^{-5}-10^{-8}$, whereas $K$ for abrasive wear is typically $10^{-1}-10^{-3} \cdot[15]$

\subsubsection{Relation between wear and friction}

According to the adhesive formulation, the magnitudes of both friction and wear coefficients are consequences of related plastic strength phenomena. The friction coefficient of a sliding pair is directly proportional to the average interfacial junction shear strength, while the wear coefficient is a measure of the probability that the shear strength of the material around a junction is less than the interfacial shear strength. Because of their similar origins several attempts have been made to more 
closely relate friction and wear phenomena. One of the more successful of these is a model proposed by Rabinowicz, which attributes wear to statistical variations in the junctions shear strengths. ${ }^{[16]}$ Using plausible assumptions regarding typical variations in both interfacial and junction shear strengths, he derives a wear coefficient vs friction coefficient plot which shows good agreement with previously obtained experimental data. When presented on a log-log plot, the wear coefficients obtained during adhesive wear studies generally show good correlation with the friction coefficients raised to approximately the third or fourth power. ${ }^{[17,18]}$

$$
K \propto \mu_{a}^{\sim 3-4}
$$

\subsection{Experimental verification of adhesive model at $4.2 \mathrm{~K}$}

Experimental evidence is presented in this section to support the extension of the adhesive theory of friction and wear to cryogenic temperatures. ${ }^{[19,20]}$ There are two key components of this investigation. The first describes st procedures used to characterize the indentation hardness of the test materiais. These hardness values are used to facilitate subsequent wear coefficient calculations. During the second phase of the investigation, a pin-on-disk sliding apparatus is used to collect representative friction and wear data for a number of polymer-on-metal sliding pairs at temperatures of $4.2,77$, and $293 \mathrm{~K}$.

Polymeric materials are emphasized for several reasons. The first is the widespread use of polymers in cryogenic applications. Polymer-on-metal sliding pairs are commonly used because of their low wear rates, and low-moderate friction coefficients. ${ }^{[21]}$ By contrast, metal-metal pairs are seldom used because of the high friction and wear that results under these poorly lubricated conditions; surface welding occurs readily for metal pairs slid in liquid nitrogen ${ }^{[2]}$ and the highfriction results produced during small-amplitude sliding in liquid helium are very similar to observed during high-vacuum sliding at $293 \mathrm{~K}$. ${ }^{[\theta]}$ The use of fluid-and grease-lubricated surfaces is not possible at cryogenic temperatures because all conventional liquid lubricants freeze. Similarly, because most cryogens are non-polar low-molecular-weight molecules, they possess low inherent lubricity, hence lubrication by the cryogens is not possible either. Polymeric materials are also widely used as insulators and structural material in superconducting magnets. Fabric- 
reinforced plastics are particularly favored for structural applications because of their good low-temperature fracture toughness, high strength-to-weight ratios, and low thermal conductivities.

The polymeric materials tested during this investigation are classified into two major types: 1) unfilled, and 2) fabric- and particle-filled plastics. The bulk of the study focused on the unfilled materials. Single-phase materials are often emphasized in studies of this sort because they have uniform properties, are easier to characterize, and because proportionally more comparative data are available in the literature. The reinforced polymers, on the other hand, are all widely used in cryogenic applications, hence their tribological properties are of general interest from a design standpoint. Since most polymers contract considerably and become increasingly brittle upon cooling to cryogenic temperatures, these fabric- and particle-fillers are used to improve the polymer's dimensional stability, load bearing capacity, and fracture toughness

\subsubsection{Description of the test materials}

The following section presents brief descriptions of the materials used during this investigation. The mechanical properties of these materials are generally well known at room temperature and to a lesser extent at cryogenic temperatures. ${ }^{[22-26]}$

\subsubsection{Metal counterfaces}

Only two types of metal counterfaces have been used during the sliding experiments. These were selected because of their widespread acceptance in cryogenic applications.

AISI 304 stainless steel was used as the disk material during most of the tests. It is a nominally nonmagnetic, low thermal conductivity alloy often used as a structural material in cryostats and other cryogenic equipment.

1/2-hard oxygen-free high-conductivity (OFHC) copper was also used, to simulate the behavior of copper-stabilized superconductors.

\subsubsection{Unfilled polymers}

Teflon, polytetrafluoroethylene, is an inert, semicrystalline polymer that re- 
mains ductile at cryogenic temperatures where it is often used in electrical insulators, O-rings, and seals. Self-lubricating, glass-fiber-reinforced Teflon bearing retainers are also used in high-speed, cryogenic turbopumps. Teflon in particularly favored in this application because of its ability to act as a solid lubricant. As sliding commences, a coherent, low shear strength film of transferred polymer forms on the opposing surface, leading to a marked reduction in the friction coefficient. ${ }^{[2,3,10,27]}$

$U H M W$, ultra-high-molecular-weight polyethylene, is a tough, abrasionresistant thermoplastic with molecular weight of approximately $4 \sim 6$ million. UHMW bearings are used where durability and low friction are desirable; however, the range of available injection molded shapes is limited due to its high melt viscosity.

$H D P E$, high density polyethylene, is a straight-chained, highly-crystalline polymer whose low melting point $(410 \mathrm{~K})$ limits its use as a bearing material to moderate loads, speeds, and temperatures.

Polypropylene is a tough, low-density, semicrystalline thermoplastic frequently used in injection-molded containers because of its low cost and limited chemical reactivity.

Kel-F, polychlorotrifluoroethylene, is an inert, flame-resistant semicrystalline polymer frequently used in highly-reactive liquid oxygen environments. It is preferred to Teflon in many applications because of its greater low-temperature fracture toughness.

Nylon 101 is the strongest and stiffest of the unmodified nylons. It is used extensively at room temperature due to its high abrasion resistance and moderate friction coefficient. Nylon 101 is a semicrystalline polymer which readily absorbs moisture in proportion to the environmental humidity, leading to considerable variability in its mechanical properties.

Lucite, polymethylmethacrylate, is an amorphous, optically-transparent polymer generally chosen more for its optical clarity than its mechanical strength. It is brittle at room temperature. Its optical properties do not deteriorate even upon prolonged outdoor exposure. 


\subsubsection{Fabric- and particle-filled polymers}

Rulon $A$ and Rulon $L D$ are particle-reinforced fluoropolymers that are used in cryogenic seals and bearings. Rulon LD is stiffer at room temperature and designed to minimize deflection under heavy load.

Nylatron GS consists of molybdenum-disulphide-filled nylon 101; it offers slightly improved mechanical and thermal properties over the unfilled nylon. The molybdenum disulphide filler is used to increase load-bearing capacity and to reduce the nylon's sensitivity to absorbed moisture.

Vespel SP-3 consists of a semicrystalline polyimide filled with $\sim 5$ vol.\% molybdenum-disulphide. Because it incorporates multiple bonds along its chain backbone, Vespel has excellent resistance to thermal degradation and retains much of its stiffness at elevated temperatures. Vespel's thermal contraction to cryogenic temperatures is smaller than most other polymers and its low outgassing rate makes it attractive for high vacuum applications.

Stycast 2850 is a general-purpose, low-temperature adhesive consisting of an aluminum-oxide-filled epoxy resin manufactured by Emerson \& Cuming, Inc. It is often used at cryogenic temperatures to provide vacuum-tight seals on laboratory apparatus and as an encapsulant for wire feed-throughs.

Phenolic $L E$ is a laminated composite consisting of woven linen in a thermosetting phenolic resin. Because of its laminated structure it has good dimensional stability and mechanical toughness at cryogenic temperatures.

$G-10$ is a glass-fabric-reinforced epoxy resin laminate used extensively as a structural support in cryogenic systems, particularly in large superconducting magnets. It is manufactured by soaking layers of glass cloth in a thermosetting resin and cured at elevated temperatures and pressures.

\subsubsection{Mechanical characterization of the test materials}

Indentation hardness measurements were used to characterize the plastic flow strengths of the test materials at both $293 \mathrm{~K}$ and $77 \mathrm{~K}$. The measurements were conducted using the modified Rockwell superficial hardness tester shown in Figure 4.1. The Rockwell tester was selected for two reasons; it provides a convenient means 
for applying the test load to the $2.4 \mathrm{~mm}(3 / 32 \mathrm{in})$ ball used as an indentor during these tests, and it is equipped with a dial gauge which monitors the indentation depth during testing. To start a test, the indentor is pressed against the test surface with a $30 \mathrm{~N}$ minor load to provide firm contact, while the tester's dial gauge is set to a reference mark. A lever which applies the major, or test, load to the sample is then engaged for $10 \mathrm{~s}$ to produce the hardness indentation. During the indentation time the dial gauge is monitored to ensure that the loading device remains within its normal working range. After the indentor load is removed, the indentation diameter is measured with an optical microscope. The Brinell hardness of the sample, $B$, is then calculated by dividing the major load, $L$, by the indentation area

$$
B=\frac{2 L}{\pi D\left(D-\sqrt{D^{2}-d^{2}}\right)}
$$

where the indentation area is determined from the ball diameter, $D$, and the indentation diameter, $d$. In accordance with standard practice the ratio $d / D$ was kept in the range $0.3-0.5$ between samples by adjusting the major load. Four major loads of $45,150,210$, and $300 \mathrm{~N}$ were used. Table 4.1 presents the mean values of at least five Brinell hardness indentations for each test material. During the roomtemperature tests, these values are generally repeatable to within $\pm 10 \%$ and agree well with previously published data. ${ }^{[28]}$

A specially-prepared liquid-nitrogen reservoir was mounted to the Rockwell tester to perform the $77-\mathrm{K}$ measurements. The reservoir enabled the tests to be performed with both the test sample and indentor completely immersed in liquid nitrogen. An $x-y$ table, affixed to the base of the reservoir, allows for two-dimensional positioning of the sample with respect to the indentor; this permits several indentation during a single test run. The intent of this arrangement was to provide a stable test environment for forming the indentations, and then to wait until the samples had warmed to room temperature to measure the indentation diameters. However, because of the unanticipated thermal recovery of many of the specimens' indentations, it was not possible to follow this procedure exactly. Instead, the procedure employed was to create a replica of the $77-\mathrm{K}$ indentations by interposing a thin $(\sim 18 \mu \mathrm{m})$ sheet of aluminum foil between the indentor and the test specimen. Thus, even if an indentation recovered completely, the specimen's $77-\mathrm{K}$ hardness 


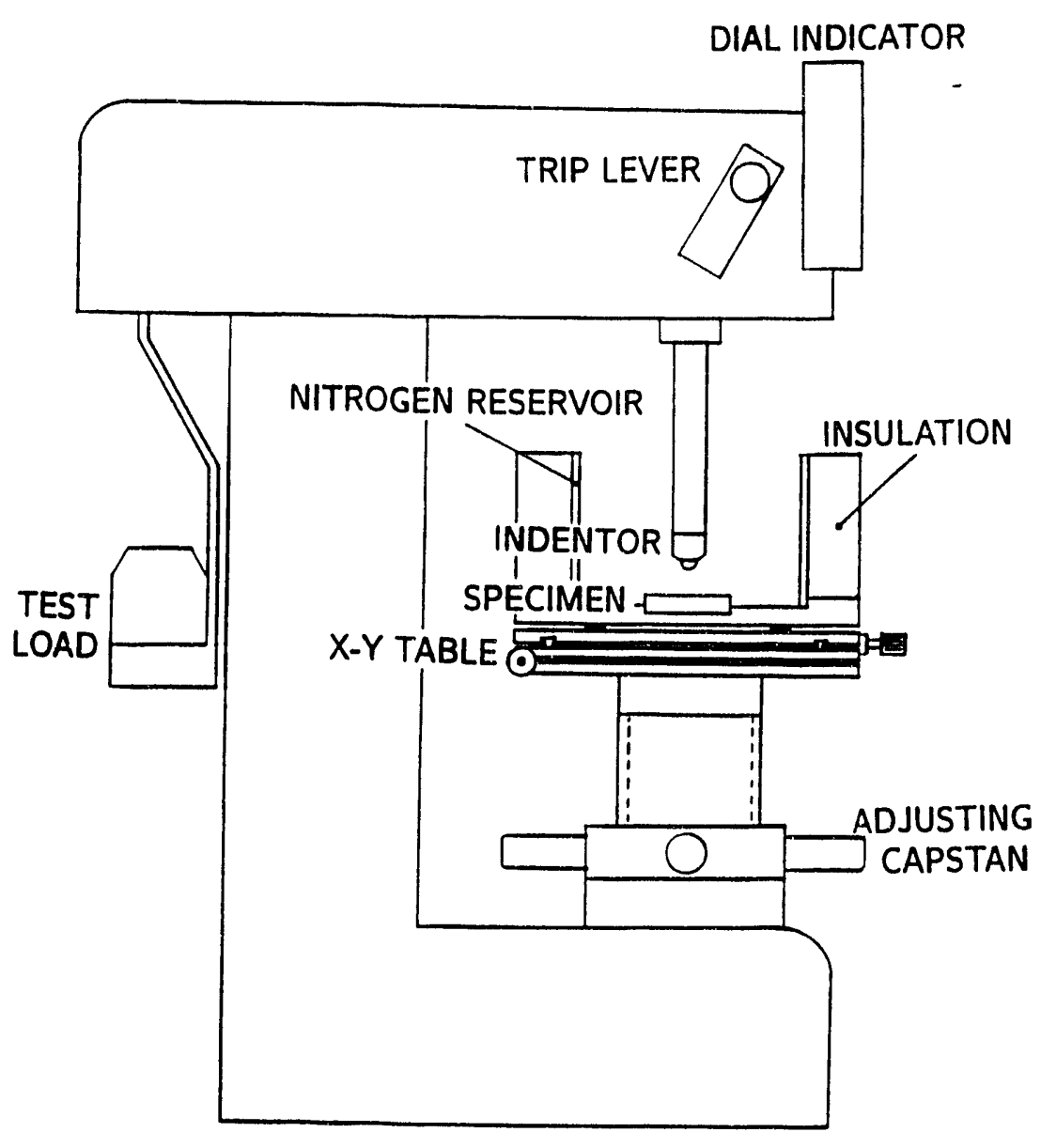

Figure 4.1: Indentation hardness test apparatus, consisting of modified Rockwell tester and liquid nitrogen reservoir. 
could still be determined by measuring the replica's indentation diameter. Aluminum foil was used for the replicas because it is readily available in thin sheets and because its yield stress is lower than that of most other structural metals; both features should minimize the replica's effect on the measured hardness value. It is interesting to note that, in contrast to the other plastics, the indentations in the most highly-crystalline samples, namely Teflon, the polyethylenes, polypropylene, and Rulon exhibit negligible recovery upon warming from $77 \mathrm{~K}$ to room temperature.

Several of the room-temperature tests were repeated, both with and without foil replication, to verify that the aluminum foil has minimal effect on the measured hardness values. During these comparative tests, results obtained using foil replicas did not vary from the conventional hardness measurements by more than $\pm 15 \%$. This accuracy is considered sufficient for wear coefficient calculations, because the statistical variation in the wear process itself is generally larger than this. ${ }^{[29]}$

Even though this hardness test procedure was not suitable for performing $4.2 \mathrm{~K}$ measurements, approximate hardness value can be estimated, from the relation between the material's penetration hardness, $P$, and its compressive yield strength, $\sigma_{Y} ;$ for a non-strain hardening material: ${ }^{[28]}$

$$
P \simeq 3 \sigma_{Y}
$$

A similar relation holds for strain-hardening materials, this time with $\sigma_{Y}$ equated to the flow stress at $0.8 \%$ strain, the equivalent plastic strain produced during indentation.

Table 4.2 presents comparative hardness values for the unfilled polymers at $4.2,77$, and $293 \mathrm{~K}$, obtained from both experimental measurement and calculated from the limited compressive yield stress data available in the literature. ${ }^{[22,23,25]}$ Compressive properties are especially important for the evaluation of potentially brittle materials, such as the polymers at $4.2 \mathrm{~K}$, because they more accurately reflect the high hydrostatic compressive stress component present at the asperities. Most brittle materials fracture at very low tensile strains; however, they can often show considerable ductility in compression. ${ }^{[30]}$

The mechanical properties of polymeric materials can vary widely, based on 
Table 4.1: 10-s Brinell Hardness Values in MPa

for Materials tested at 77 and $293 \mathrm{~K}$.

\begin{tabular}{|c|c|c|}
\hline Material & Hardness at $77 \mathrm{~K}$ & Hardness at $293 \mathrm{~K}$ \\
\hline Teflon & 450 & 33 \\
\hline UHMW & 520 & 48 \\
\hline HDPE & 550 & 62 \\
\hline Polypropylene & 590 & 90 \\
\hline Kel-F & 640 & 120 \\
\hline Nylon 101 & 750 & 150 \\
\hline Lucite & 740 & 200 \\
\hline Rulon $A$ & 490 & 41 \\
\hline Rulon LD & 530 & 43 \\
\hline Nylatron GS & 730 & 140 \\
\hline Vespel SP-3 & 500 & 190 \\
\hline Stycast 2850 & 1220 & 240 \\
\hline Phenolic LE & 1030 & 420 \\
\hline G-10 & 1280 & 530 \\
\hline OFHC Copper & 800 & 550 \\
\hline 304 Stainless steel & 3120 & 1760 \\
\hline
\end{tabular}

Table 4.2: Brinell Hardness Values

for Various Polymeric Materials at Low Temperatures.

\begin{tabular}{|r||r|r||r|r|r|}
\hline \multicolumn{1}{|c||}{} & \multicolumn{3}{c||}{$\begin{array}{c}\text { Measured Hardness } \\
\text { Material }\end{array}$} & \multicolumn{3}{c|}{$\begin{array}{c}\text { Hardness (MPa) Calculated from } \\
\text { Compressive Yield Stress Data }\end{array}$} \\
\hline \multicolumn{1}{|c||}{} & at $77 \mathrm{~K}$ & at $293 \mathrm{~K}$ & at $4.2 \mathrm{~K}$ & at $77 \mathrm{~K}$ & at $293 \mathrm{~K}$ \\
\hline & \multicolumn{1}{|c||}{} & & & & \\
\hline Teflon & 450 & 33 & $540^{a}, 490^{b}$ & $370^{a}, 390^{b}$ & $20^{a}, 41^{b}$ \\
\hline UHMW & 520 & 48 & $510^{c}$ & - & $120^{d}$ \\
\hline Kel-F & 640 & 120 & $580^{d}$ & $510^{d}$ & $120^{e}, 270^{f}$ \\
\hline Vespel Sp-3 & 500 & 190 & $470^{e}, 770^{f}$ & $380^{e}, 670^{f}$ & \\
\hline
\end{tabular}

${ }^{a}$ Ref. [23], p.99

${ }^{c}$ Ref. [22], p.326

${ }^{b}$ Ref. [25], Fig. G.3.a-1

'Ref. [23], p.656

${ }^{d}$ Ref. [23], p. 350

${ }^{f}$ Ref. [23], p.647, flow stress at $0.8 \%$ strain 
their processing methods, nevertheless, the good correlation in Table 4.2 between the experimental hardness results and those calculated from previously published data suggests that our hardness test method is a viable means for obtaining lowtemperature mechanical data. The available data likewise indicate that $\sigma_{y}$ rises by only $20 \sim 50 \%$ between 77 to $4.2 \mathrm{~K}$; this observation was applied during the adhesive wear studies to justify the use of $77 \mathrm{~K}$ hardness values for calculating $4.2 \mathrm{~K}$ wear coefficient.

\subsubsection{Pin-on-disk experiments}

Friction and wear experiments were run to examine the steady-state sliding mechanisms of polymer-metal pairs, using a rotational pin-on-disk apparatus developed during an earlier investigation. ${ }^{[6]}$. Three test environments were used: 1) at $293 \mathrm{~K}$ in room temperature air, 2) at $77 \mathrm{~K}$ with the samples immersed in liquid nitrogen, and 3 ) at $4.2 \mathrm{~K}$ in liquid helium.

\subsubsection{Apparatus and procedures}

Figure 4.2 presents a schematic cross-section of the pin-on-disk apparatus used during the friction-velocity experiments. The test geometry consisted of a rotating metal disk slid against three hemispherically-ended polymer pins, symmetrically spaced on a $51-\mathrm{mm}$ bolt circle diameter. Symmetrically-spaced pins were used to make the apparatus self-aligning and to permit the simultaneous testing of three specimens under identical test conditions. By concentration the specimen contact into a limited number of spots, the relatively simple pin-on-disk geometry facilitates a rapid comparative evaluation of a number of material pairs under different environmental conditions. The apparatus was designed to operate in air at room temperature and at cryogenic temperatures with the friction surfaces immersed in the appropriate cryogenic liquid.

The apparatus is capable of operating at nominal sliding speeds between $10^{-7}$ and $10^{-1} \mathrm{~m} / \mathrm{s}$, using a velocity controlled servo motor and various combinations of precision gear reducers. However, during the wear experiments the nominal sliding speed at the pins was fixed at $2 \times 10^{-2} \mathrm{~m} / \mathrm{s}$ and the normal load at $22.5 \mathrm{~N}$. During a test the accumulated sliding distance was calculated using a rotational mechanical counter coupled to the apparatus drive shaft. All tests were conducted 


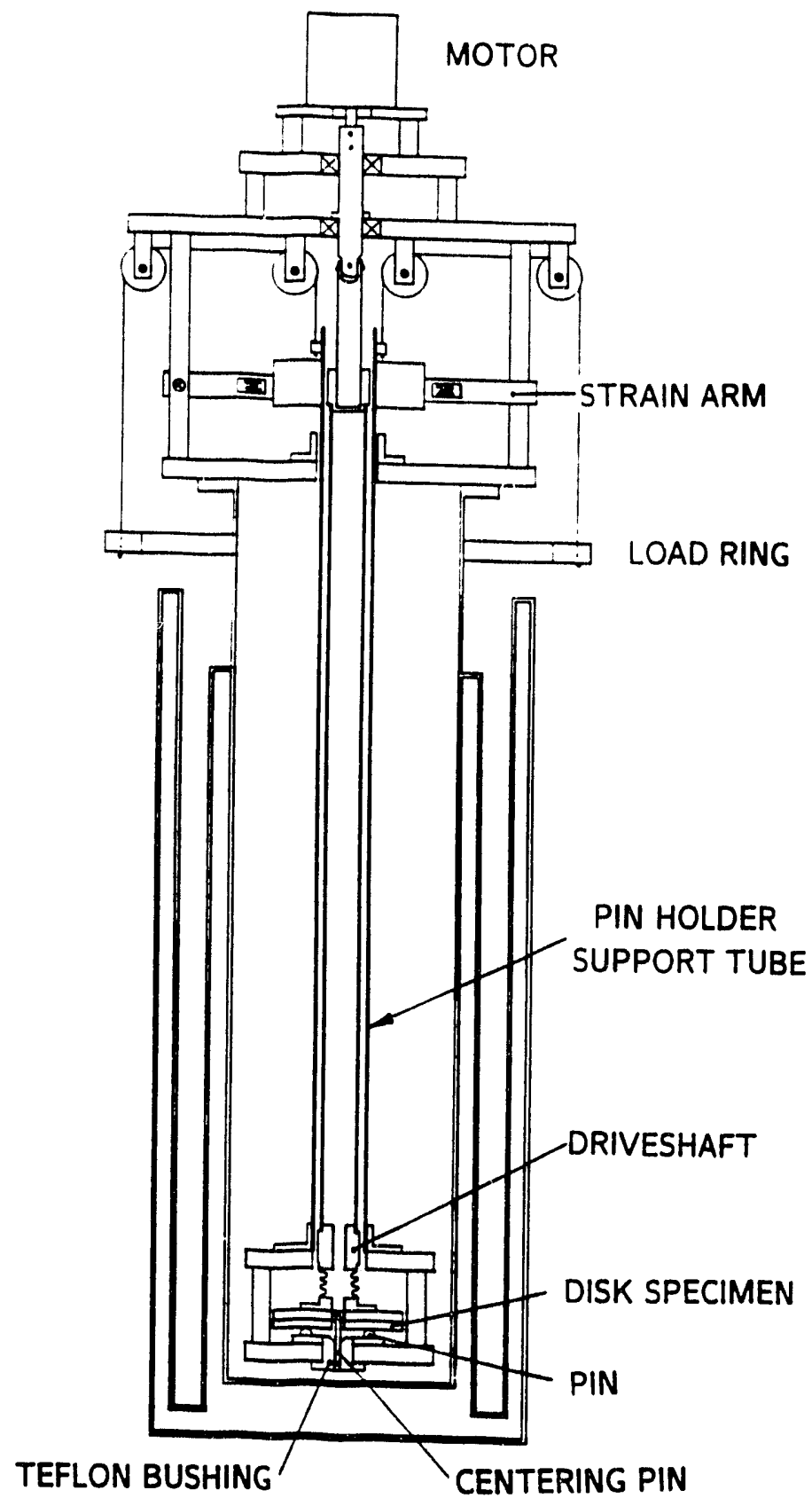

Figure 4.2: Schematic cross-section of the pin-on-disk apparatus. 
for a minimum sliding distance of $1.5 \mathrm{~km}$.

The friction coefficient is measured by strain arms which prevented the rotation of the outer friction assembly. These friction values are monitored continuousiy during testing using a computer-based data acquisition system. The pins' wear volumes are determined at the conclusion of each test from optical measurements of the wear scar diameters. Because of the pins' geometry, relatively minute amounts of wear can be determined by comparing the wear scar diameter, $d$, to the pin's radius of curvature, $R$. It can be shown that that wear volume, $V$ may be given in terms of $d$ and $R:[10]$

$$
V \simeq \frac{\pi d^{4}}{64 R}\left(1+\frac{d^{2}}{12 R^{2}}\right)
$$

$R$ was $3.18 \mathrm{~mm}$ during these tests, and the wear scar diameters were determined to the nearest $25 \mu \mathrm{m}$, as the average value of two orthogonal measurements for each pin. Although the use of rounded pins results in highly localized contact between the sliding surfaces, the friction coefficients obtained under these conditions are usually valid for less concentrated geometries because the friction coefficient of a sliding pair is usually independent of the apparent contact area. ${ }^{[12]}$

The cryogenic experiments were performed by first lowering the pin-on-disk tester into its cryostat. To prevent any condensation on the test specimens during cool-down the cryostat was vigorously purged by pumping heliurn gas through a tube that extended to the bottom of the apparatus. To prevent influx of roomtemperature air into the cryostat, flexible rubber seals were installed between the test assembly and the cryostat mounting flange. A sliding fit was used to minimize frictional interaction between the disk's drive shaft and the rest of the apparatus. Air infiltration during the liquid-helium tests was further reduced by providing a small inflow of helium gas to maintain a slight, positive pressure in the cryostat. Heat conduction to the specimens was minimized by suspending the test specimens approximately $1 \mathrm{~m}$ below the cryostat mounting flange. The boil-off gas was likewise vented through the apparatus to further reduce the heat influx. The apparatus' quiescent helium boil-off rate was in the range $0.10 \sim 0.15 \ell / \mathrm{h}$. During a liquidhelium wear test the actual boil-off rate depended largely on the frictional energy dissipation and was approximately $0.35 \ell / h$ for a friction coefficient of 0.3 ; this typically allowed for at least $24 \mathrm{hr}$ operation for a single helium transfer. 


\subsubsection{Sample Preparation}

Most of the commercial plastics were obtained as $6.35 \mathrm{~mm}(1 / 4 \mathrm{in})$ diameter rods and turned to their final shape using a radius form tool. 'The Lucite, polypropvlene. and many of the Teflon samples were obtained as $6.35 \mathrm{~mm}(1 / 4 \mathrm{in})$ diameter balls. The Stycast pins were formed by casting into a specially prepared specimen mold. During processing, the epoxy was outgassed in a vacuum lower than $200 \mathrm{mtorr}$ both before and after molding to remove any air which may have been trapped in the resin during compounding. Before a test, three of the desired pins were mounted in a brass specimen holder, wiped with a clean swab soaked in methanol, and allowed to air dry before being installed in the test apparatus.

The metal disks were machined from plate stock and faced with a single point cutting tool to remove surface irregularities. The disks were then randomly abraded against 320-grit silicon carbide paper under running water; this was done both to clean the disks and to provide a consistent surface finish between tests. The surface roughness of the disks prior to test, as measured by a commercial profilometer, was typically in the range $0.38-0.42 \mu \mathrm{m}(15-17 \mu \mathrm{in})$. Because water will not wet a surface covered with organic contaminants, the disks were considered clean enough for testing when a water layer wet the entire disk surface. The disks were then rinsed with methanol to displace the water layer and air dried on clean tissue paper.

\subsubsection{Experimental results}

The results of the pin-on-disk sliding experiments are presented in Tables 4.3 and 4.4. Table 4.3 shows the results for the unfilled polymers, and Table 4.4, the results for the fabric- and particle-filled plastics. Two types of metal disks were used during the experiments. Although several preliminary runs were performed against OFHC copper the most frequently used disk specimen was AISI 304 stainless steel. Because no statistical differences were observed in the results for the two materials, the counterface material is not explicitly listed in the data tables.

Two sets of data are included in each of Tables 4.3 and 4.4. The first indicates the arithmetic mean and \pm one standard deviation of the time-averaged friction coefficients and the second, the geometric mean and \pm one standard deviation of the wear coefficients. Because wear coefficient values generally follow a log-normal 
distribution the standard deviation is represented as a factor of the mean value $( \pm f(-))$. A wear coefficient entry of $9 \pm f(2)$ then would indicate a range of data geometrically distributed between 4.5 and 18 with a mean value of 9 .

To verify the reliability of the apparatus and the repeatability of the test results several test combinations were run more than once. The number of repeat tests ranged from two to six, with three being most typical. Statistical summaries of these tests are included in Tables 4.3 and 4.4 . The friction coefficients were repeatable to within $\pm 10-15 \%$ while the wear coefficients were reproducible to within a factor of about 2.5. These values are consistent with the variation typically observed during laboratory adhesive wear testing and can be used to indicate materials and environments which give exceptionally low or high friction and wear values.

\subsubsection{Unfilled polymers}

All of the polymers listed in Table 4.3 were tested against 304 stainless steel. A few of the polymers, Teflon, polypropylene and Lucite, were also tested against OFHC copper. However, because there were no statistical differences in the results for the polymers tested against both materials, these results are combined as a single entry in the table.

Figure 4.3 shows a crossplot of the wear coefficients $v s$ the corresponding friction coefficients for the unfilled polymers. ${ }^{[20]}$ The plotting symbols in this figure have been partially shaded to differentiate between test temperatures. The solid line in the figure represents the results of a linear regression analysis of all the data, conducted on the assumption that the friction coefficient was the error-free variable. The line has a slope of 3.0 on the log-log plot and a correlation coefficient of 0.81 . The correlation of the friction and wear results to a single regression line suggests that the wear mechanism is probably the same in all cases. In addition, both the magnitude of the wear coefficients, typically in the range $5 \times 10^{-7}$ to $5 \times 10^{-6}$, and the third-power relation between friction and wear implicate adhesive wear as the principal wear mechanism.

The good overall correlation of the test results is slightly surprising, considering the wide range of test temperatures used and the approximations inherent in the hardness measurements. However, since the adhesive wear theory was originally 
Table 4.3: Test Results for the Unflled Polymer Pins.

\begin{tabular}{|c|c|c|c|}
\hline & \multicolumn{3}{|c|}{ Friction Coefficient } \\
\hline Material & at $4.2 \mathrm{~K}$ & at $77 \mathrm{~K}$ & at $293 \mathrm{~K}$ \\
\hline Teflon & $0.16 \pm .01$ & $0.09 \pm .0 \mathrm{i}$ & $0.12 \pm .01$ \\
\hline UHMW & $0.12 \pm .01$ & $0.07 \pm .02$ & $0.15 \pm .01$ \\
\hline HDPE & $0.14 \pm .02$ & $0.11 \pm .03$ & $0.18 \pm .01$ \\
\hline Polypropylene & $0.24 \pm .01$ & $0.21=.03$ & $0.22 \pm .04$ \\
\hline Kel-F & $0.21 \pm .03$ & $0.19 \pm .01$ & $0.36 \pm .01$ \\
\hline Nylon 101 & $0.10 \pm .02$ & $0.09 \pm .01$ & $0.28 \pm .02$ \\
\hline \multirow[t]{4}{*}{ Lucite } & $0.50 \pm .06$ & $0.34 \pm .01$ & $0.30 \pm .03$ \\
\hline & \multicolumn{3}{|c|}{ Wear Coefficient } \\
\hline & \multicolumn{3}{|c|}{$k \times 10^{8}$} \\
\hline & at $4.2 \mathrm{~K}$ & at $77 \mathrm{~K}$ & at $293 \mathrm{~K}$ \\
\hline & & & \\
\hline Teflon & $1.0 \pm f(1.9)$ & $0.8 \pm f(2.2)$ & $5.0 \pm f(1.6)$ \\
\hline UHMW & $0.2 \pm f(1.9)$ & $0.4 \pm f(1.8)$ & $0.3 \pm f(2.1)$ \\
\hline HDPE & $1.2 \pm f(3.3)$ & $2.3 \pm f(1.6)$ & $0.9 \pm f(1.9)$ \\
\hline Polypropylene & $8.0 \pm f(1.6)$ & $18.0 \pm f(1.4)$ & $4.9 \pm f(2.3)$ \\
\hline Kel-F & $3.4 \pm f(2.8)$ & $10.4 \pm f(2.7)$ & $5.2 \pm f(2.9)$ \\
\hline Nylon 101 & $0.4 \pm f(1.9)$ & $0.2 \pm f(2.3)$ & $2.2 \pm f(1.4)$ \\
\hline Lucite & $220.0 \pm f(1.2)$ & $65.0 \pm f(2.7)$ & $25.0 \pm f(1.5)$ \\
\hline
\end{tabular}

Note: for the wear coefficients one standard deviation is represented as being within a certain factor $\pm f(-)$ of the mean value. 
Table 4.4: Test Results for the Fabricand Particle-Reinforced Polymer Pins.

\begin{tabular}{|c|c|c|c|}
\hline & \multicolumn{3}{|c|}{ Friction Coefficient } \\
\hline Material & at $4.2 \mathrm{~K}$ & at $77 \mathrm{~K}$ & at $293 \mathrm{~K}$ \\
\hline D.hor & & & \\
\hline Kuion A & C.30 & 0.19 & 0.10 \\
\hline Rulon LD & 0.27 & 0.18 & $0.21 \pm .01$ \\
\hline Nylatron GS & $0.13 \pm .02$ & $0.10 \pm .01$ & . iv $\therefore \quad 13$ \\
\hline Vespel SP-3 & 0.08 & $0.21 \pm .01$ & $0.3 \mathrm{t}$ \\
\hline Stycast 2580 & 0.35 & $0.41 \pm .03$ & 0.29 \\
\hline Phenolic LE & 0.44 & 0.40 & $0.44 \pm .05$ \\
\hline \multirow[t]{4}{*}{ G-10 } & $0.21 \pm .01$ & $0.51 \pm .04$ & 0.32 \\
\hline & \multicolumn{3}{|c|}{ Wear Coefficient } \\
\hline & \multicolumn{3}{|c|}{$k \times 10^{6}$} \\
\hline & at $4.2 \mathrm{~K}$ & at $77 \mathrm{~K}$ & at $293 \mathrm{~K}$ \\
\hline & & & \\
\hline Rulon $\mathrm{A}$ & 4.9 & 1.8 & 0.04 \\
\hline Rulon LD & 4.5 & 2.7 & $0.08 \pm f(1.1)$ \\
\hline Nylatron GS & $0.2 \pm f(2.5)$ & $0.3 \pm \mathrm{f}(2.1)$ & $2.0 \pm f(1.1)$ \\
\hline Tespel SP-3 & 1.1 & $0.8 \pm f(2.0)$ & 0.7 \\
\hline Sitycast 2580 & 52.0 & $54.0 \pm \mathrm{f}(1.2)$ & 5.0 \\
\hline Phenolic LE & 8.0 & 11.0 & $3.2 \pm f(2.7)$ \\
\hline G-10 & $0.7 \pm f(3.3)$ & $67.0 \pm f(2.3)$ & 3.4 \\
\hline
\end{tabular}

Note: for the wear coefficients one standard deviation is represented as being within a certain factor $\pm f(-)$ of the mean value. 


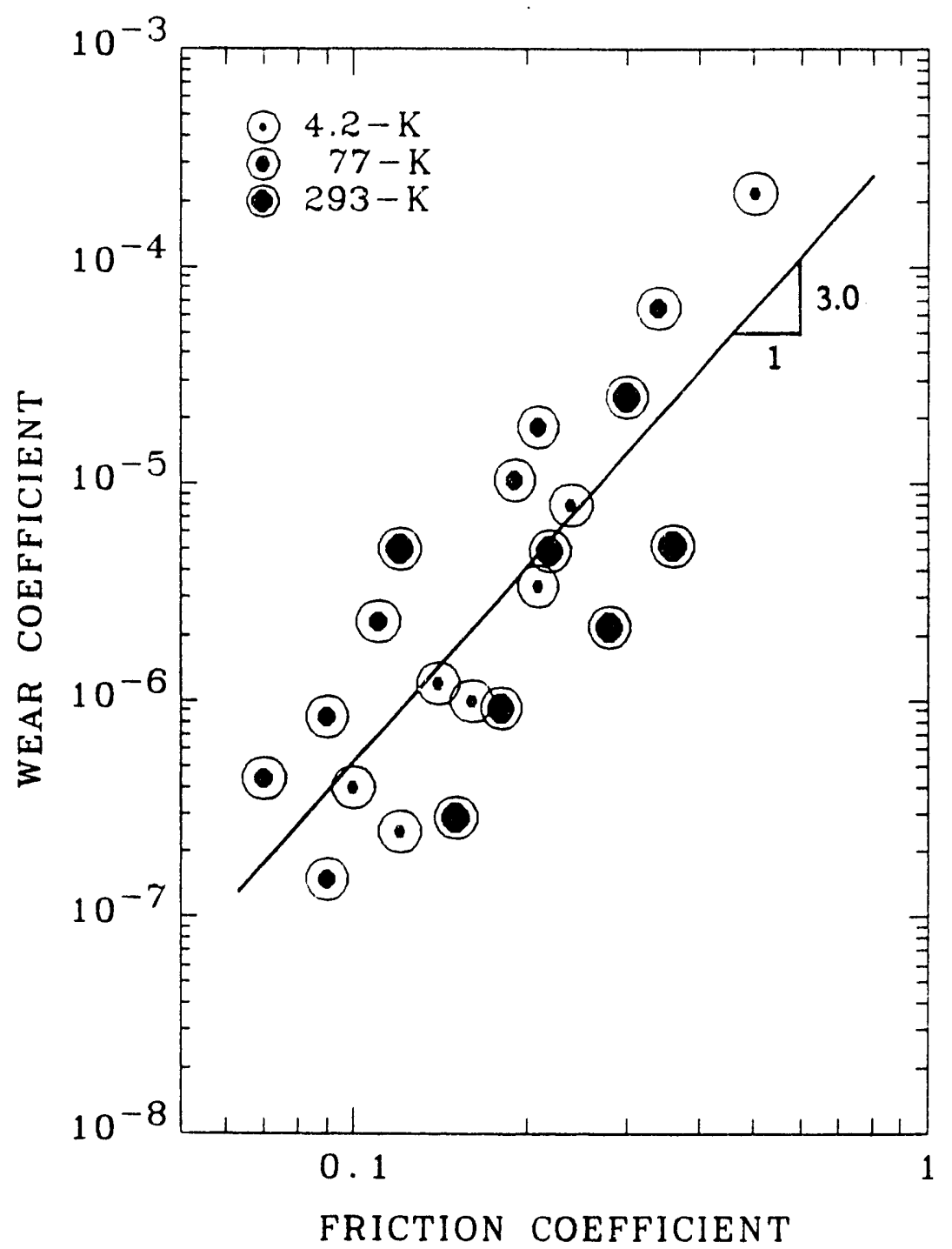

Figure 4.3: Average wear coefficients $v s$ friction coefficients for the unfilled polymers. The best least-squares fit of the data has a slope of 3.0 on the $\log$-log scale. Figure from [20]. 
formulated to interpret the behavior of rigid-plastic materials, the direct extension of the adhesive theory to cryogenic temperatures was not entirely unanticipated. At these low temperatures, the polymers' deformation mode is primarily plastic. whereas they become increasingly viscoelastic-plastic above room temperature.

As a rule, the polymers in Table 4.3 that possess polar repeat units exhibit larger friction coefficients than the nonpolar polymers; this tendency is in agreement with the asperity-junction model. ${ }^{[11]}$ The non-polar molecules' low dipole forces effectively limits their interfacial shear strengths; this reduces not only their friction coefficients but also their wear coefficients, since this low interfacial strength helps to promote shear along the original contacting surfaces.

Over the range of temperatures investigated, UHMW consistently demonstrates the lowest friction and wear coefficients of the polymers. UHMW's favorable sliding characteristics are predicated on its unique combination of chemical and structural properties; its low friction results because of its low polarity and limited chemical reactivity, while its low wear is attributed to its very high polymer-chain length. ${ }^{[31,32]}$ There are principally two barriers to the formation of polymeric wear particles. One is the energy necessary to rupture individual polymer chains, while the second is the energy necessary to draw the existing chain ends out from the polymer bulk. ${ }^{[10]}$ UHMW has few pre-existing chain ends, hence, its low wear rate results most probably because of the higher stresses needed for molecular chain scission. The low-friction/low-wear behavior of nylon at the cryogenic temperatures is also quite unexpected; it may perhaps be attributed to the strengthening effect of hydrogen bonding between the molecules at low temperatures.

\subsubsection{Fabric- and particle-flled polymers}

Figure 4.4 presents a crossplot of the wear coefficient $v s$ friction coefficient data for the fabric- and particle-filled polymers. ${ }^{[20]}$ Among these materials, the Rulons, Nylatron, Vespel, Phenolic and G-10 samples were tested against 304 stainless steel while the Stycast was tested against OFHC copper. The solid line in Figure 4.4 represents the results of a linear-regression analysis performed on the assumption that the friction coefficient was the error-free variable. The line has a slope of 3.4 on the $\log -\log$ plot and a correlation coefficient of 0.80 . Both the slope of this line 
and the magnitude of the wear coefficients in Table 4.4 suggest that adhesive wear was the dominant wear mechanism during these experiments as well.

A case for the moderate adhesive wear of the reinforced plastics can likewise be made based on the nature of the sliding specimens and relative reactivities of the test environments. Many of the fillers examined during this part of the study are mildly abrasive; the Rulons consist of Teflon compounded with hard inorganic fillers, G-10 is filled with woven glass fibers, and Stycast, with aluminum oxide. During repeated sliding against a metal counterface the fillers slowly abrade away the oxides and adsorbed surface films that are typically present on most metal surfaces.

By providing the metal with a less-active surface layer, these adsorbed films generally reduce the metal's tendency towards very-strong adhesive bonding. ${ }^{[33]}$ Because these films do have such a profound influence on the asperity interactions which occur during sliding, the cleaning procedures implemented during this type of study are intended to provide an easily reproducible, grease-free surface layer composition from test to test rather than to produce atomically clean surfaces.

At room temperature many of these surface films are constantly reformed during sliding, for example due to the absorption of water vapor from the environment, until a steady-state surface composition is reached; this effect is often responsible for the characteristic run-in behavior observed for many sliding systems. However, during sliding in inert liquid nitrogen or liquid helium environments the surface layers do not usually reform to any significant extent. The removal of these layers often leads to the observed formation of very clean, finely polished metallic wear tracks and is accompanied by a corresponding increase in interfacial adhesion. ${ }^{[\theta]}$ Abrasive fillers can likewise increase friction and wear by precluding the development of selflubricating polymeric transfer films. ${ }^{\text {[27] }}$ Thus, the effect of mildly abrasive fillers on increasing the surface activity of a sliding pair provides a plausible model for the increased low-temperature friction and wear of many of the filled polymers.

Solid-lubricant-filled polymers like Vespel and Nylatron are often used to overcome the uncertainty in surface layer composition by providing a transferred layer of known composition. During sliding, it is frequently observed that certain ma- 


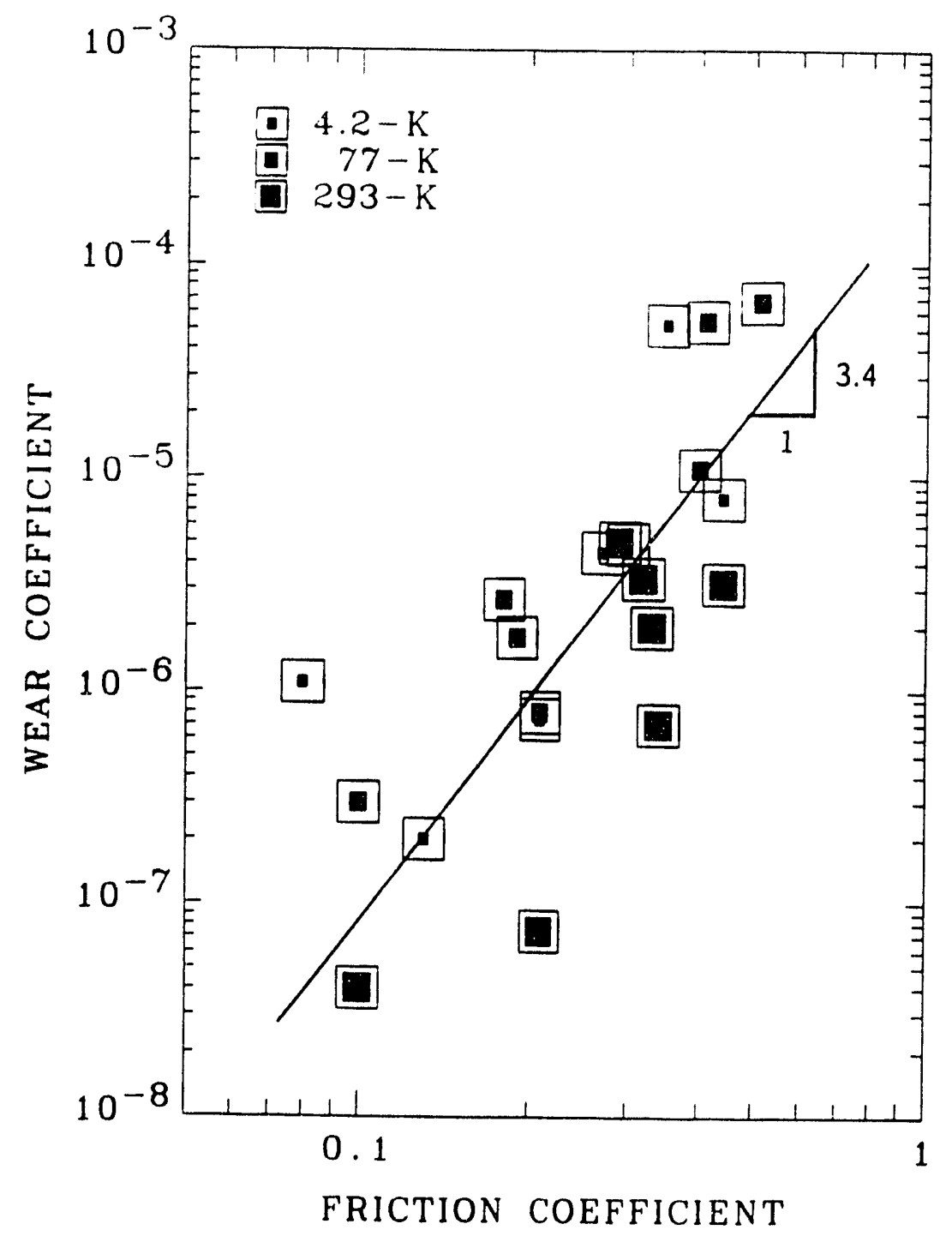

Figure 4.4: Average wear coefficients $v s$ friction coefficients for the fabric- and particlereinforced polymers. The best least-squares fit of the data has a slope of 3.4 on the log-log scale. Figure from [20]. 
terials like graphite, carbon, Teflon, and molybdenum disulphide transfer to the opposing surface as a thin, strongly adherent film. As Eq. 4.4 indicates, as long as the shear strength of this film, $s_{l}$, is less than the junction's unlubricated shear strength, $s$, friction should be reduced. However, there is considerable debate concerning the effectiveness of solid lubrication for relatively soft materials like thermoplastic polymers, whose friction is already low. ${ }^{[10,31]}$ This debate is born out by the temperature-dependent friction and wear coefficients for Nylatron, which are almost identical to those for the unfilled nylon (Table 4.3).

\subsection{Theoretical summary of experimental data}

The experimental results presented in this chapter strongly suggest that the adhesive theory of polymer friction and wear remains directly applicable at cryogenic temperatures. A major advancement in the present investigation was its emphasis on determining the polymers' low temperature hardnesses. Prior studies have attributed the dramatic reduction in most polymers' low-temperature wear rates to completely separate high-temperature/low-temperature wear mechanisms. ${ }^{[4]}$ However, this investigation clearly demonstrates that the polymers' wear coefficients, and hence their principal wear mechanism, remain fairly constant as the temperature is changed and that their wear rates are reduced at cryogenic temperatures because of their much larger low-temperature hardnesses.

Based on the analysis of the experimental data, interfacial adhesion has been identified as a fundamental mechanism in the cryogenic tribology of polymer-metal pairs. There are several methods for modifying the frictional characteristics of a sliding pair available within the framework of the adhesive theory. Thus, the identification of interfacial adhesion as a major factor in low-temperature tribology permits a much more systematic approach to the selection of low-temperature sliding materials; this approach will be more fully developed in Chapter 6 . 


\section{References for Chapter 4}

1. Simon, I., McMahon, H.O., and Bowen, R..J. Dry metallic friction as a function of temperature between $4.2 \mathrm{~K}$ and $600 \mathrm{~K} \mathrm{~J}$. Appl. Phys. 22 (1951) 177-184.

2. Wisander, D.W., Maley, C.E., and Johnson, R.L. Wear and friction of filled polytetrafluoroethylene compositions in liquid nitrogen ASLE Trans. 2 (1959) 5866.

3. Rhempe, W.H., Jr. Research and development of materials for use as lubricants in a liquid hydrogen environment ASLE Trans. 9 (1966) 213-221.

4. Glaeser, W.A., Kissel, J.W. and Snediker, D.K. Wear mechanisms of polymers at cryogenic temperatures Polymer Science and Technology $5 \mathbf{B}$ (ed. Lee, L.H.), Plenum Press, New York (1974) 651-662.

5. Iwabuchi, A., Honda, T. and Tani, J. Tribological properties at temperatures of 293, 77 and $4 \mathrm{~K}$ in fretting Cryogenics 29 (1989) 124-131.

6. Kensley, R.S., and Iwasa, Y. Frictional properties of metal insulator surfaces at cryogenic temperatures Cryogenics 20 (1980) 25-36.

7. Kragelskii, I.V. Friction and Wear Buttersworth, Washington (1965) p 34-35, 54-57.

8. Rabinowicz, E. Autocorrelation analysis of the sliding process J. Appl. Phys. 27 (1956) 131-135.

9. Jain, V.K., and Bahadur, S. Development of a wear equation for polymermetal sliding in terms of the fatigue and topography of the sliding surfaces Wear 60 (1980) 237-248.

10. Lancaster, J.K. Friction and wear, Ch. 14 in Polymer Science (ed. Jenkins, A.D.), North-Holland Publishing, Amsterdam (1972) 960-1046.

11. King, R.F., and Tabor, D. The effect of temperature on the mechanical properties and the friction of plastics Proc. Roy. Soc. B66 (1953) 728-736.

12. Rabinowicz, E. Friction and Wear of Materials John Wiley, New York (1965). 13. Archard, J.F. Contact and rubbing of flat surfaces J. Appl. Phys. 24 (1953)
981-988.

14. Rabinowicz, E. Wear coefficients - metals Wear Control Handbook (ed. Peterson, M.B., and Weiner, W.O.), American Sociely of Mechanical Engineers, NY (1980) 475-478.

15. Rabinowicz, E. Tribology II: Advances in Friction, Lubrication, and Wear Center for Advanced Engineering Study, MIT (1983) Figure 2-8.

16. Rabinowicz, E. An adhesive wear model based on statistical variation in strength 
values Wear 63 (1980) 175-181.

17. Rabinowicz, E. Advances in Wear-resistant Materials ASME Publication $72-\mathrm{DE}$ 28, New York (1972).

18. Michael, P.C., Saka, N., and Rabinowicz, E. Burnishing and adhesive wear of an electrically conductive polyester-carbon film IVear 132 (1989) 265-285.

19. Coleman, K. Friction and Wear of Polymers at Cryogenic Temperatures S.B. Thesis, Dept. of Mechanical Engineering, MIT (1989).

20. Michael, P.C., Rabinowicz, E. and Iwasa, Y. Friction and wear of polymeric materials at 293, 77, and 4.2 K Cryogenics 31 (1991)

21. Tobler, R.L. A review of antifriction materials and design for cryogenic environments Adv. Cryo. Eng. 26 (1980) 66-77.

22. Scott, R.B. Cryogenic Engineering (1963 ed.) Reprinted 1988 by Met-Chem Research, Boulder, CO.

23. Schramm, R.E., Clark, A.F., and Reed, R.P. A Compilation and Evaluation of Mechanical, Thermal, and Electrical Properties of Selected Polymers National Bureau of Standards Monograph 132 (1973).

24. Wigley, D.A. Materials for Low-temperature Use Oxford University Press, Great Britian (1978).

25. Cryogenic Materials Data Handbook Air Force Materials Laboratory, WrightPatterson Air Force Base, OH (1970).

26. Swenson, C.A. Mechanical properties of Teflon at low temperatures Rev. Sci. Instr. 25 (1954) 834-835.

27. Nosaka, M., Oike, M., Kamijo, K. and Kikuchi, M. Experimental study of lubricating performance of self-lubricating ball bearings for liquid hydrogen turbopumps Lubrication Engineering 44 (1988) 30-44.

28. McClintock, F.A., and Argon, A.S. Mechanical Behavior of Materials Addison-Wesley, Reading, MA (1966) p 443-468.

29. Rabinowicz, E. Friction, Wear, and Lubrication: Tribology I Center for Advanced Engineering Study, MIT (1974) p 1.9-1.11.

30. McClintock, F.A., and Argon, A.S. op. cit. p 490-495.

31. Pratt, G.C. Plastic-based bearings Lubrication and Lubricants (ed. Braithwaite, E.R.), Elsevier Publishing, New York (1967) .

32. Anderson, J.C. High density and ultra-high molecular weight polyethylenes: their wear properties and bearing applications Tribology 15 (1982) 43-47.

33. Peace, J.B. Solid lubricants Lubrication and Lubricants (ed. Braithwaite, E.R.), Elsevier Publishing, New York (1967) . 


\section{Conductor motion disturbances}

Chapter 5 reviews the contemporary status of conductor-motion research in drywound superconducting solenoids. Three major kinds of studies are discussed. The earliest studies examined the frictional characteristics of common superconductorinsulator pairs to identify combinations that exhibit the most favorable sliding characteristics at $4.2 \mathrm{~K} .{ }^{[1-4]}$ The second approach concentrated on evaluating the quench characteristics of individual conductors and small-scale winding models. Activities under this approach include the: introduction of the $\mathrm{AE} /$ voltage technique for monitoring abrupt conductor motions, ${ }^{[5,6]}$ development of conductor motion modelling, ${ }^{[7-11]}$ experimental determination of the slip conditions necessary for quench initiation, ${ }^{[12-14]}$ and verification of the most probable quench-initiation locations in single layer winding models. ${ }^{[15,16]}$ As conductor-motion-induced disturbances became better understood, methods for improving the reliability of drywound magnets have been proposed. The third type of study examines the application of these methods to full-scale dry-wound solenoids. ${ }^{[15-18]}$

\subsection{Frictional characterization of cryostable magnets}

\subsubsection{Motivation}

By the mid-1970s it had become clear the Stekly criterion ${ }^{[19]}$ for absolute cryostability was much more conservative than needed for most applications. Magnets designed according to this criterion provide sufficient cryogenic cooling capacity to permit the entire magnet to recover from the normal to the superconducting state following a quench. Given this extreme cooling capacity, the prospect of a single disturbance capable of instantaneously quenching the entire magnet is rather small. For a cooling capacity less than that proscribed by the Stekly criterion, it is more commonly assumed that normal zones up to a certain maximum size will recover to the superconducting state, whereas normal zones larger than this will grow. ${ }^{[20]}$

The boundary between normal zone growth and recovery is known as the min- 
imum propagating zone (MPZ); this MPZ concept was previously discussed with regard to "adiabatic" windings in $\$ 3.1 .3 .2$. The energy required to raise the MPZ volume from its operating temperature to its critical is known as the magnet's minimum quench energy. If the maximum disturbance energy in a winding could be determined analytically, the task would then become one of matching the magnet's cooling capacity to this disturbance energy. The earliest investigations of superconductor/insulator friction ${ }^{[1-4]}$ were performed as part of a larger effort to analytically predict the magnitudes of the maximum disturbances likely to be encountered during magnet operation. ${ }^{[21]}$ In a sense, these studies constituted a very preliminary approach towards the quasi-adiabatic design mentioned in $§ 3.1 .2$.

There are many advantages to eliminating excess cooling capacity. ${ }^{[22]}$ The first is that any space previously occupied by cooling channels could be replaced by conductors. This increases the volume fraction of superconductor in the winding and allows for a reduction in the overall winding volume. In addition, by eliminating void spaces, the winding can be more structurally robust. Since the conductors are now more tightly packed together they can support proportionally more of their electromagnetic stresses; this further reduces the need for additional structural supports in the winding.

\subsubsection{Superconductor-insulator friction}

A main source of disturbance energy in dry-wound superconducting magnets is frictional heating caused by abrupt conductor motions. In cryostabilized magnets the motion occurs along insulating spacers that are used both to support the conductors and to form the cooling channel boundaries. Because the frictional heat generation is proportional to the product of the friction force times the sliding velocity, the friction-velocity characteristics of superconductor-insulator pairs plays a key role in determining the maximum disturbance energy.

Two series of experiments were performed to determine the frictional characteristics of common superconductor-insulator pairs at $4.2 \mathrm{~K}$. The first used a pin-ondisk friction apparatus to measure the friction coefficient $v s$ velocity characteristics of several superconductor insulator pairs at 300,77 , and $4.2 \mathrm{~K} .{ }^{[1,2]}$ The second series employed a linear-type friction apparatus at $4.2 \mathrm{~K}$ to study the transition from 
static contact to macroscopic sliding. ${ }^{3,4]}$

\subsubsection{Pin-on-disk experiments}

\subsubsection{Test results}

The operation of the pin-on-disk apparatus is described in $\$ 4.2 .3 .1 .6 .35 \mathrm{~mm}$ (1/4") oxygen-free-high-conductivity (OFHC) copper balls were used during these experiments to simulate multifilamentary, composite superconductors whose outer surfaces are also essentially all copper. The $6.35 \mathrm{~mm}$ thick disk specimens consisted commercial plastics that are often used in cryogenic applications; they included: Teflon, nylon, polyethylene, G-10, and Phenolic LE. Brief descriptions of these materials are provided in $\S 4.2 .1$.

Figure 5.1 presents stylized plots of the three most frequently proposed frictionvelocity diagrams. ${ }^{[23,24]}$ Examples of each of the three sliding types were observed at room temperature, however, at cryogenic temperatures only those characteristics corresponding to Figures $5.1 \mathrm{~b}$ and $5.1 \mathrm{c}$ were observed. ${ }^{[1,2]}$ Figure 5.2 shows representative friction-velocity traces for copper/insulator pairs at each of the three test temperatures. Figure 5.2a was obtained for a copper/nylon pair, while Figure 5.2b was for a copper/G-10 pair.

Figure 5.1a depicts a universal friction characteristic postulated for all materials by the creep theory of friction. ${ }^{[24]}$ The theory contends that the velocity-dependence of the friction coefficient reflects the interfacial creep behavior of the sliding system in shear. Points to the left of the maximum friction coefficient indicate the strain rate sensitivity of the interfacial shear stress, while points to the right of the maximum demonstrate the breakdown of this creep mechanism. Experimental evidence suggests that it takes a finite time for an interface to grow to full strength. ${ }^{[25]}$ Hence, as the sliding speed increases to the right of the friction maximum, the friction coefficient decreases because of the limited time allowed for interfacial strengthening. ${ }^{[24]}$

Figure $5.1 \mathrm{~b}$ depicts the sliding characteristics for a material whose maximum friction coefficient lies so near the ordinate of zero velocity that only the descending portion of the friction-velocity curve can be experimentally observed. ${ }^{[24]}$ A plausible explanation for the widespread occurrence of this friction behavior at cryogenic temperatures is that the steady-state creep characteristics of all materials are severely 

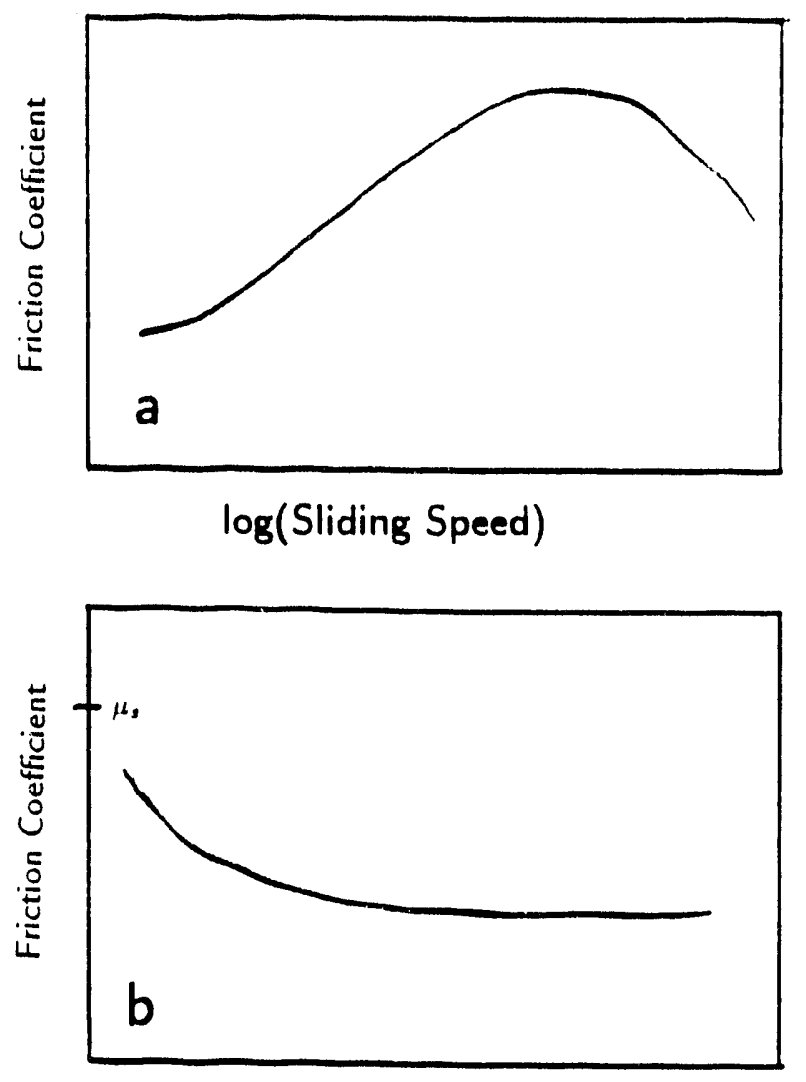

Sliding Speed

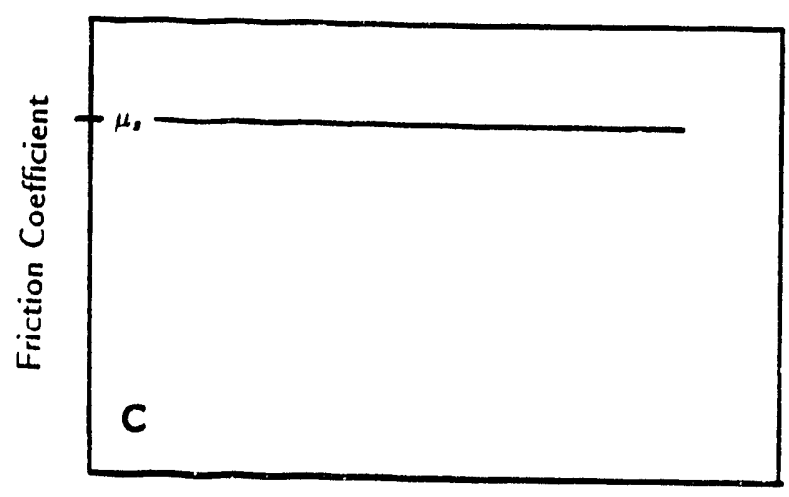

Sliding Speed

Figure 5.1: Friction-velocity diagrams. a) A universal friction-velocity trace postulated for all materials by the creep theory of friction. b) Variation on trace $a$ in which maximum friction value occurs so near to zero velocity that only the descending portion of the curve can be observed in practice. c) Near constant friction coefficient observed for inert, creep-resistant materials. 
rather, the sliding speed increases gradually as the friction force increases. Frictiunal stabilization is especially important at cryogenics temperatures, where heat capacities are low, because the energy dissipation rate during conductor motion is much easier to control by limiting the sliding velocity than by limiting the friction coefficient. For all of the insulators examined the friction coefficient varied by less than one order of magnitude, from around 0.05 to 0.5 . By contrast, the sliding velocity can potentially vary over several orders of magnitude.

If a given disturbance energy could be distributed over a longer duration its effect on the winding's thermal stability could be reduced considerably. ${ }^{[12]}$ Results presented in $§ 3.1 .3 .2$ indicate that as the time scale of the disturbance passes from 1-100 ms, steady-state conduction contributes increasingly towards the winding's heat transfer characteristics. Although the search for inherently stable friction materials continues, ${ }^{[28]}$ no material has yet been identified which possess a positive friction-velocity characteristic at $4.2 \mathrm{~K}$. ${ }^{[2 \theta]}$

Given the present lack of inherently stable $4.2-\mathrm{K}$ friction materials, the present alternative is to rely on marginally stable materials, represented by the friction trace in Figure 5.1c. G-10 is a prime example of this type of material. The $4.2-\mathrm{K}$ friction behavior of G-10 is reasonably well characterized. However, these friction characteristics are not sufficient to accurately determine the energy dissipation during conductor motion. The details of motion, namely, the sliding speed, friction force, and extent of motion are also determined by the conductors' overall stress state, loading rate, stiffness, local mechanical support, and winding tolerance; efforts to incorporate these effects into existing conductor-motion models are presently under development in other laboratories. ${ }^{[\theta-11,14]}$

\subsubsection{Linear friction experiments}

\subsubsection{Linear friction apparatus}

Because of the limited extent of motion possible in a superconducting magnet, transient friction experiments have also been performed to more closely simulate sliding initiation in superconductor-insulator pairs. ${ }^{[3,4,12,2 \theta]}$ These tests are typically performed using a linear-type friction apparatus. Figure 5.3 presents a schematic illustration of one of these machines. ${ }^{[28]}$ In this arrangement, an OFHC cripper block 
reduced as the sliding temperature is lowered. ${ }^{20 \mid}$ This account is consistent with the experimental results obtained for the nylon (Figure 5.2a), polyethylene, and 'Teflon $\mathbb{B}_{\text {sampiec, }}{ }^{[2]}$ At room temperature all three materials are well known to exhibit velocity-dependent friction characteristics similar to that depicted in Figure $5.1 \mathrm{a}$, while at $4.2 \mathrm{~K}$ all three exhibit friction characteristics much closer to Figure $5.1 \mathrm{~b}$. At $4.2 \mathrm{~K}$ the static friction coefficients, $\mu_{s}$, for these materials are roughly twice the value of their kinetic friction coefficients, $\mu_{k}$, which were typically in the range $0.10-0.15 .^{[2]}$

The friction characteristics presented in Figure 5.1c are generally observed for relatively-inert, creep-resistant materials. During the $4.2 \mathrm{~K}$ friction experiments, this behavior was observed during sliding against laminated composites like G10 (Figure 5.2b), a glass-fabric-reinforced epoxy, and Phenolic LE, a linen-clothreinforced phenolic. Both materials are widely used in cryostable magnets as cooling channel spacers, structural supports, and electrical insulators.

\subsubsection{Macroscopic sliding stability}

From the standpoint of magnet stability, Fig'sre 5.1b presents the least desirable friction behavior for a superconductor-insulator pair. During the initiation of sliding the large drop from the static to the kinetic friction coefficient produces a local instability known as a stick-slip. The rapid, accelerated motion which accompanies a stick-slip produces an intense burst of local heating. Measurements performed on small scale winding models have verified the unstable slips with a velocity on the order of $\sim 0.1 \mathrm{~m} / \mathrm{s}$ generate ample amounts of heat to induce a quench. ${ }^{[4,5,13]}$ Hence, the implementation of material selection guideline designed to prevent stick-slip have since produced a marked improvement in the overall reliability of cryostable magnets. ${ }^{[12]}$

Materials with positive friction-velocity characteristics have long be en used at room temperature to guarantee macroscopic sliding stability. ${ }^{[27]}$ A key component of these superconductor-insulator studies was the search for materials which possess a positive slope to their friction-velocity plots, $d \mu / d v>0$, at $4.2 \mathrm{~K}$ over a range of velocities up to $10^{-4} \sim 10^{-3} \mathrm{~m} / \mathrm{s}^{[1,2,4]}$ Because the friction coefficient varies continuously with velocity there is no abrupt transition from static contact to motion, 

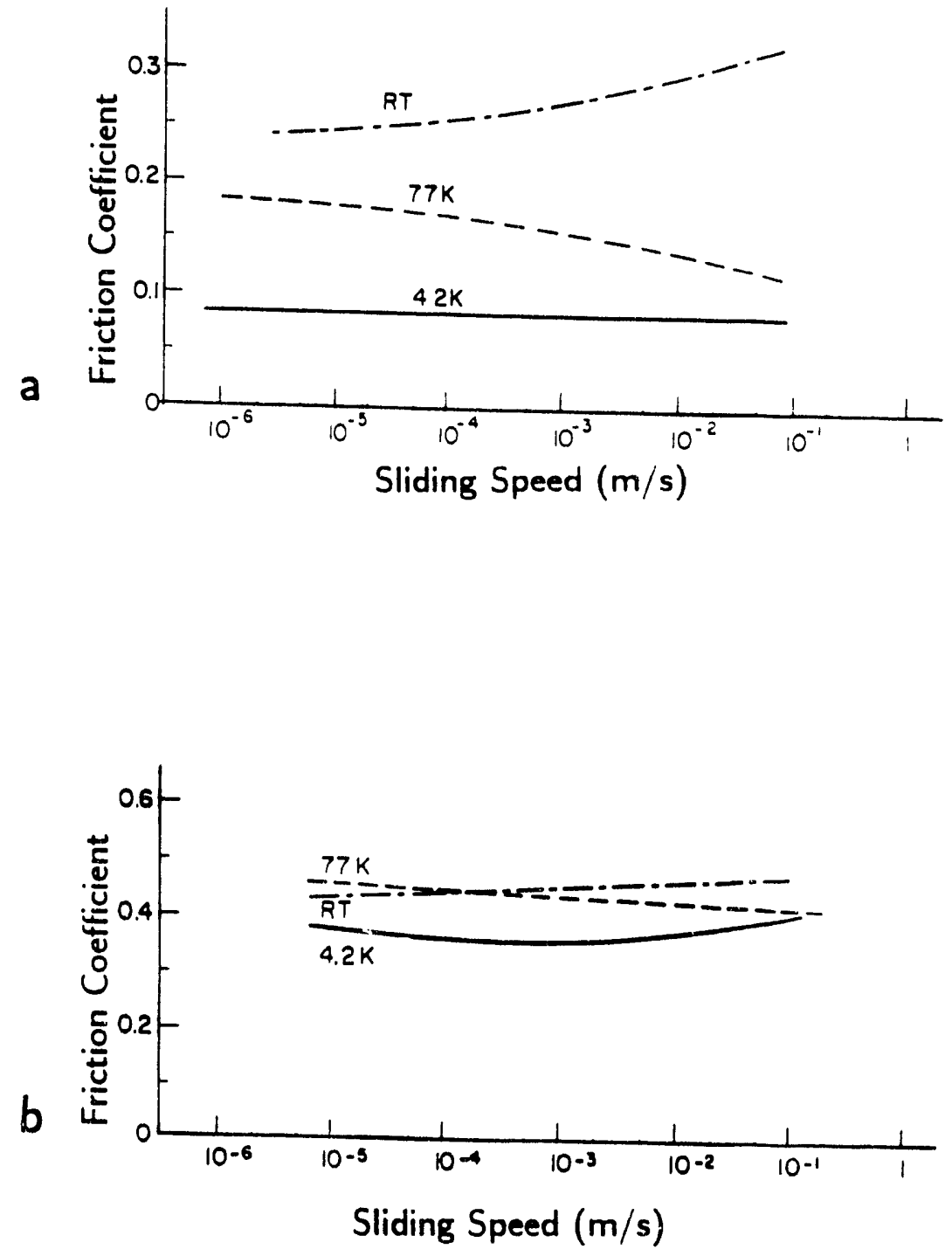

Figure 5.2: Friction-velocity traces from at pin-on-disk at each for the three test temperatures for a) copper/nylon and b) copper/G-10 sliding pairs. Figures from [2]. 


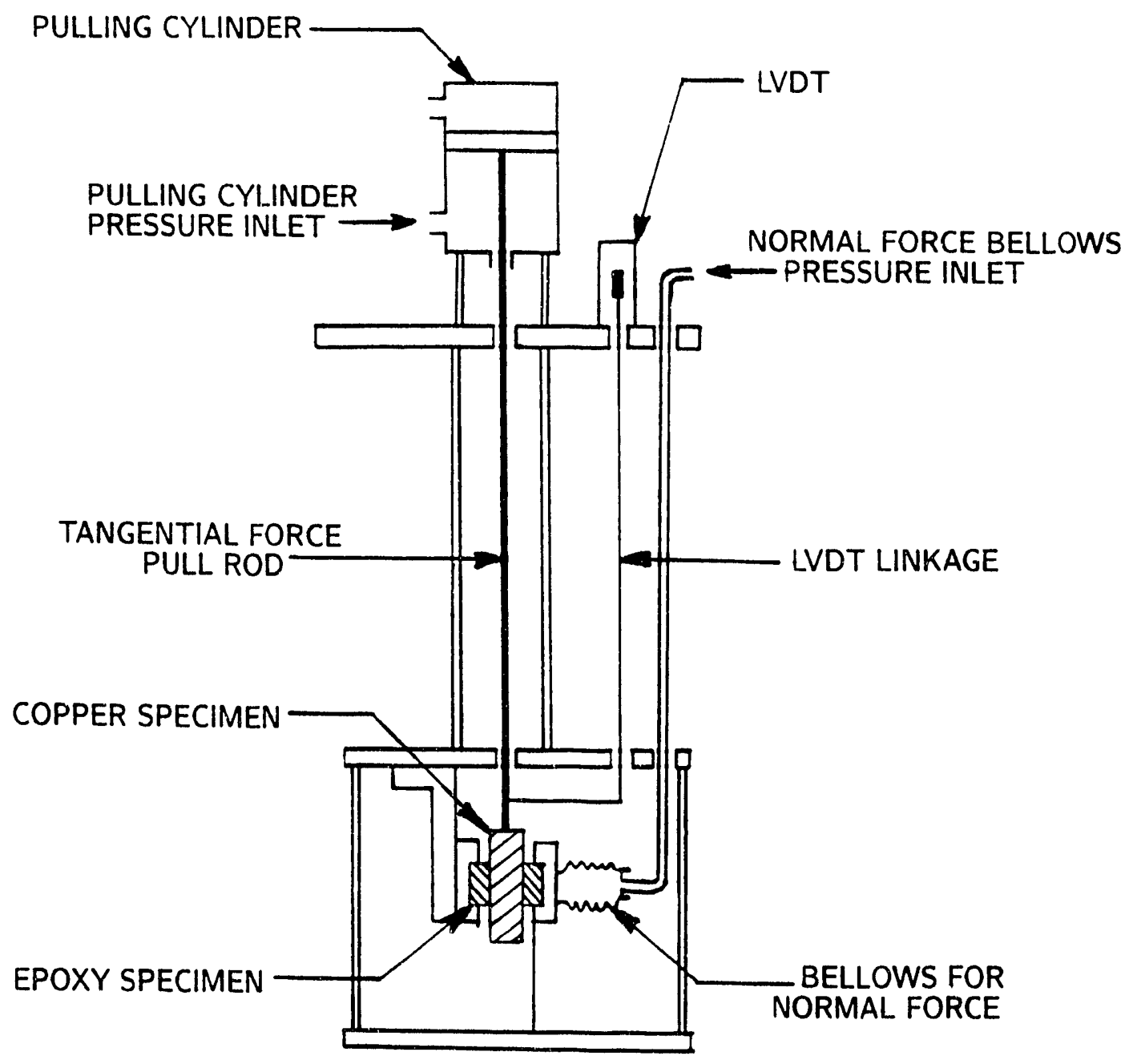

Figure 5.3: Schematic illustration of linear friction apparatus used during transient slip experiments. Figure from [26]. 
was used to simulate multifilamentary composite superconductors. This block was clamped between two samples of the same insulator using a pneumatic-bellows clamping system. The insulators examined include Teflon, nylon, and G-10.

At the start of a test run the normal, or clamping, force is set to a fixed value by applying a specified pressure to the bellows. A pneumatic cylinder mounted at the top of the apparatus is used to generate the frictior, or pulling, force applied to the copper block. This pulling force is transmitted to the copper specimen by means of a stainless steel rod. A load cell mounted between this stainless steel rod and the pulling cylinder is used to monitor the friction force. During a test, the pulling force is increased (ramped) from zero at a fixed rate until slippage occurs. Because the pulling force is actuated by the application of pressurized nitrogen gas, the friction force ramping rate can be controlled by regulating the gas flow rate into the pulling cylinder with a needle valve. The clamping pressures and tangential force ramping rates are selected to approximate the stress levels encountered in large, cryostable superconducting magnets.

The displacement of the copper specimen is measured by a linear voltage differential transducer. The LVDT core is fixed to the apparatus mounting plate with a leak-tight fitting and the LVDT core is mechanically linked to the upper end of the copper slider.

\subsubsection{Friction force-displacement diagrams}

Figure 5.4 presents examples of the tangential force $v s$ displacernent traces obtained at $4.2 \mathrm{~K}$ during the transient friction experiments; these results corroborate the materials' respective tendencies towards stable, or unstable, sliding initiation. Figure 5.4a presents results for an unstable copper/nylon pair at a normal force of $2000 \mathrm{~N}$, while Figure $5.4 \mathrm{~b}$ shows data for a stable copper/G-10 pair at a normal force of $1300 \mathrm{~N}$. In these traces, the displacement up to point $\mathrm{B}$ was due largely to elastic deformation of the apparatus between the frictional contact and the measurement location. In trace $5.4 \mathrm{~b}$ macroscopic sliding occurs beyond point $\mathbf{B}$ and continues smoothly until point $\mathbf{T}$. The slope of the force-displacement curve between points $\mathbf{B}$ and $\mathbf{T}$ indicates the relative stability of the sliding pair; sliding initiation becomes progressively smoother as this slope increases. At point $\mathbf{T}$ the maximum friction 

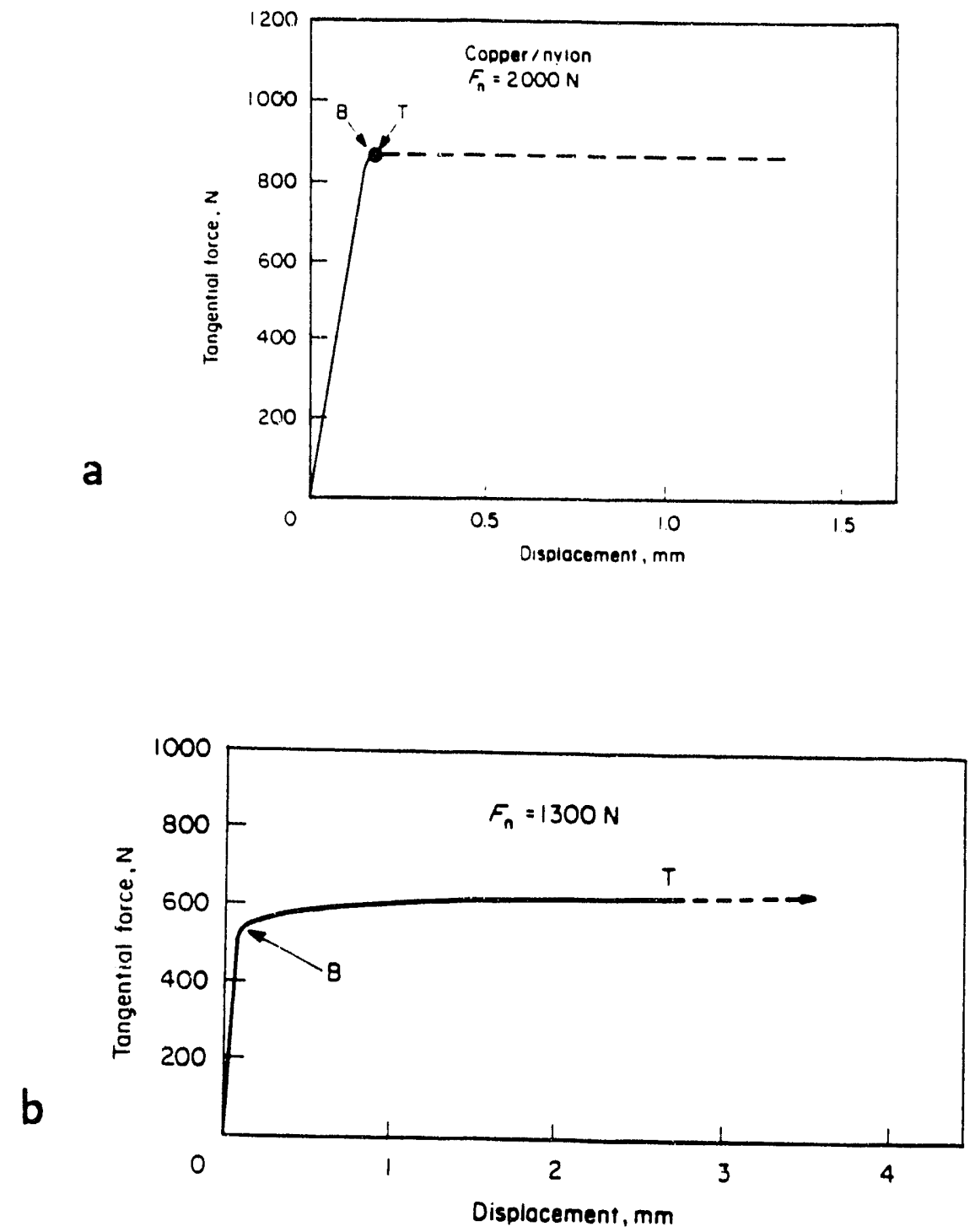

Figure 5.4: $4.2 \mathrm{~K}$ friction force-displacement diagrams. a) For an unstable copper/nylon pair at a $2200 \mathrm{~N}$ normal force. b) For a stable copper/G-10 pair at a $2200 \mathrm{~N}$ normal force. Figure from [12]. 
coefficient for the sliding pair is exceeded; this leads to an abrupt acceleration, or "take-off" of the sliding surfaces. For the unstable sliding pair in Figure 5.4a the points $\mathbf{B}$ and $\mathbf{T}$ merge; the take-off occurs before sliding begins.

A second indicator of the materials' tendencies towards unstable sliding was obtained by measuring the slopes of their displacement $v s$ time traces to determine their slip speeds just prior to take-off. Although the magnitudes of these slip speeds are peculiar to the specifics of the test arrangement, the relative ranking of the speeds corroborated the general trends observed in the friction $v s$ displacement traces. Materials with low slip speeds exhibited much larger displacements prior to take-off than those with higher speeds. ${ }^{[3]}$

During repeat tests on the same sliding pair, the friction trace often varies during the first few slip cycles; after this the slip behavior becomes largely repeatable. Figure 5.5a shows an example of this run-in effect for a copper/machined G-10 pair tested five times at a $2200 \mathrm{~N}$ normal force, while Figure $5.5 \mathrm{~b}$ shows the run-in effect for a copper/Teflon pair. The same tendencies were observed to varying degrees for all materials used in that study. ${ }^{[12]}$ Each test run $(\mathbf{A}-\mathbf{E})$ has been horizontally displaced with respect to the previous trace. During the first few slip cycles the friction coefficient decreases slightly and the slip behavior becomes progressively more stable. The progressive improvement in slip behavior with repeated cycling has been used as a partial explanation for magnet training. ${ }^{[4]}$

Recent tests at $4.2 \mathrm{~K}$ suggest that linear thermoplastic polymers like Teflon demonstrate fairly pronounced transient creep behavior only at stress levels above their elastic limits. ${ }^{[29]}$ However, upon subsequent load cycling no creep is observed until the stress is raised above the maximum stress level observed during the previous loading cycle; these results may partially explain the unstable slip behavior represented by Figures 5.5b. During the initial loading cycle, prior to the application of the friction force, transient creep increases the real contact area, thus increasing the static friction coefficient. As sliding occurs less time is available for junction growth, the friction coefficient drops, and a take-off occurs. During subsequent sliding cycles, the junction growth is not as extensive; semi-stable sliding because the friction drop is not as large. 

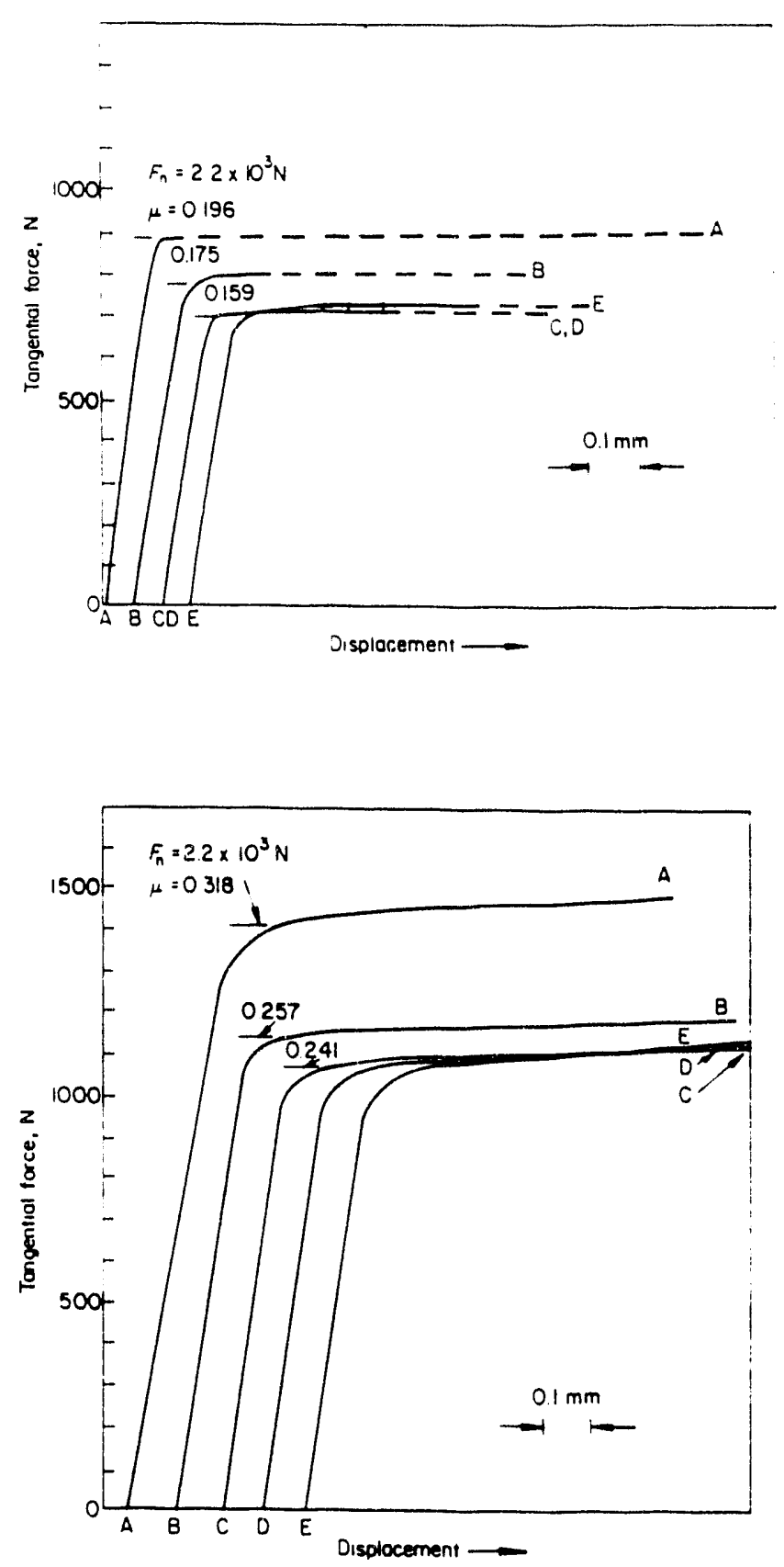

Figure 5.5: $4.2 \mathrm{~K}$ friction force-displacement diagrams during running-in of a) copper/nylon, and b) copper/G-10 pairs. Figure from [3]. 


\subsubsection{Acoustic emission during sliding}

A second rendition of the linear friction apparatus was used to monitor transient sliding phenomena at a much higher resolution. ${ }^{|12|}$ In addition to the load cell and LVDT this apparatus was also equipped with a magnetic extensometer and an $\mathrm{AE}$ sensor. The magnetic extensometer consisted of a persistent-mode superconducting Helmholtz coil and a small search coil. Relative motion between the search coil and Helmholtz coil produces a voltage at the search coil output. For small displacements this output is proportional to the relative velocity while the time integral of the voltage output is proportional to the displacement. The dynamic response of the magnetic extensometer was $\sim 10 \mathrm{kHz}$ and the spatial resolution $\sim 0.1 \mu \mathrm{m}$. A major advantage offered by the added transducers is their ability to function in a cryogenic environment; this enables them to be directly coupled tn the sliding specimens thus improving their dynamic responses. Their chief limitation is that they respond well only to impulsive events.

There are several possible methods for processing AE data. At its simplest level, an $\mathrm{AE}$ sensor can be used as an event detecior. Impulsive mechanical events produce acoustic energy which is subsequently detected by the sensor to indicate that something has happened. Additional information is generally needed to characterize the nature of the acoustic event. ${ }^{[30]}$ This added information is often encoded in a signal's spectral frequency content; for a given test configuration different types of events can sometimes be identified by their characteristic frequency responses. For instance, spectral frequency analysis has been used to discriminate between epoxyfracture and conductor motion disturbances in high-performance magnets. ${ }^{[31]}$

In certain circumstances $\mathrm{AE}$ signals are produced more or less continuously. In these instances it is more typical to analyze the entire $\mathrm{AE}$ signal rather than the individual events. Two methods are commonly used to characterize continuous AE. One is tc determine the average acoustic energy content, $\mathcal{E}_{a e}(t)$, by integrating the square of the direct AE signal, $V_{a e}^{2}(t)$, over a fixed sampling interval, $\tau$.

$$
\varepsilon_{a e}(t)=\int_{0}^{\tau} V_{a e}^{2}(t) d t
$$

The second is to electronically determine the $\mathrm{AE}$ count rate using a rate meter to record the occurrence frequency of $\mathrm{AE}$ pulses over a preset triggering level. 
Figure 5.6 presents traces of the $\mathrm{AE}$ count rate, friction force ramp rate. and LVDT output $v s$ time for a solder-coated copper/G-10 pair tested at a $2200 \mathrm{~N}$ normal force. Events occurring before $500 \mathrm{~s}$ in this trace correspond to the elastic displacement portion of the friction force-displacement curve, up to point $\mathbf{B}$, while events after $500 \mathrm{~s}$ represent macroscopic sliding. Discrete AE bursts are observed during friction force ramping prior to the onset of motion. When macroscopic motion begins at $500 \mathrm{~s}$ a large increase in the $\mathrm{AE}$ count rate was observed. This elevated $\mathrm{AE}$ count rate continues as sliding proceeds with a pulse rate proportional to the slip velocity. ${ }^{[3]}$

The correspondence between continuous AE and macroscopic sliding has been widely observed in tribological literature; each $\mathrm{AE}$ burst is usually attributed to an individual asperity interaction. ${ }^{[32-35]}$ The proportionally between the AE count rate and sliding speed is consistent with this view. The number of asperities contacts remains roughly constant during sliding, hence, an increase in speed will produce a corresponding increase in the frequency of asperity interactions. Several attempts have been made to extract additional information from these continuous AE signals regarding the number, size, and distribution of the asperity contacts. However, Briggs ${ }^{[30]}$ contends that it is unlikely that these efforts will ever succeed because of the analytical complexity of separating the direct $\mathrm{AE}$ signals, produced by an arbitrary and continuously varying asperity population, from the reflected structural resonances of the sliding specimens.

\subsubsection{Microslips}

Figure 5.7 presents an alternate representation of the copper/G-10 transient sliding experiment shown in Figure $5.2 \mathrm{~b} .{ }^{[12]}$ This plot shows direct output signals for the LVDT, magnetic extensumeter, and AE transducer $v s$ time. An interesting feature of these results is that the magnetic extensometer output (middle trace) contains many voltage pulses even when there is no discernable output from the LVDT. Each of these pulses is generally accompanied by the simultaneous occurrence of an $\mathrm{AE}$ burst. These $\mathrm{AE} /$ extensometer pulses are attributed to small slips, or microslips, whose displacement is below the LVDT resolution. Each microslip displacement during these tests, $0.5 \sim 5 \mu \mathrm{m}$, is believed to correspond to the typical asperity contact dimensions. ${ }^{[12]}$ For the limited number of pairs tested, this char- 


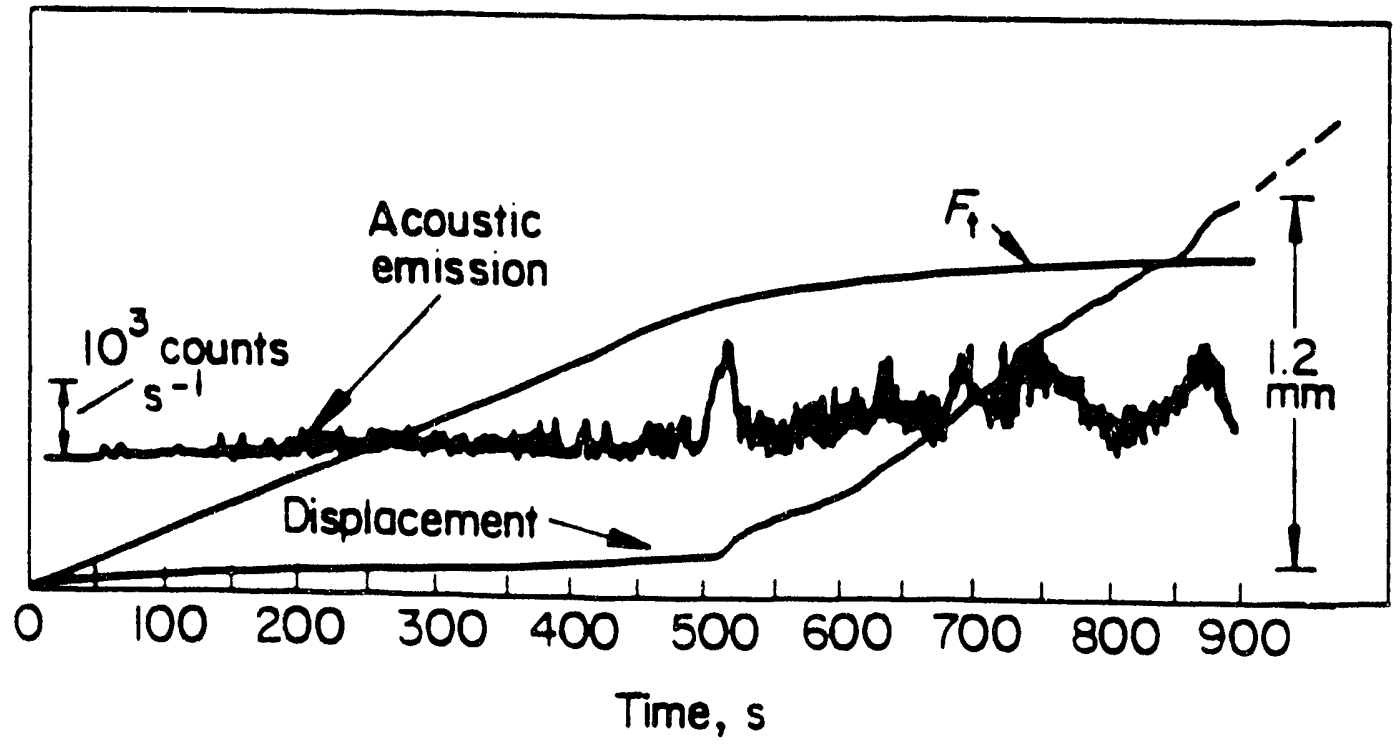

Figure 5.6: Acoustic count rate, normal force and total displacement $v s$ time for a copper/G-10 transient slip experiment. Figure from [3]. 


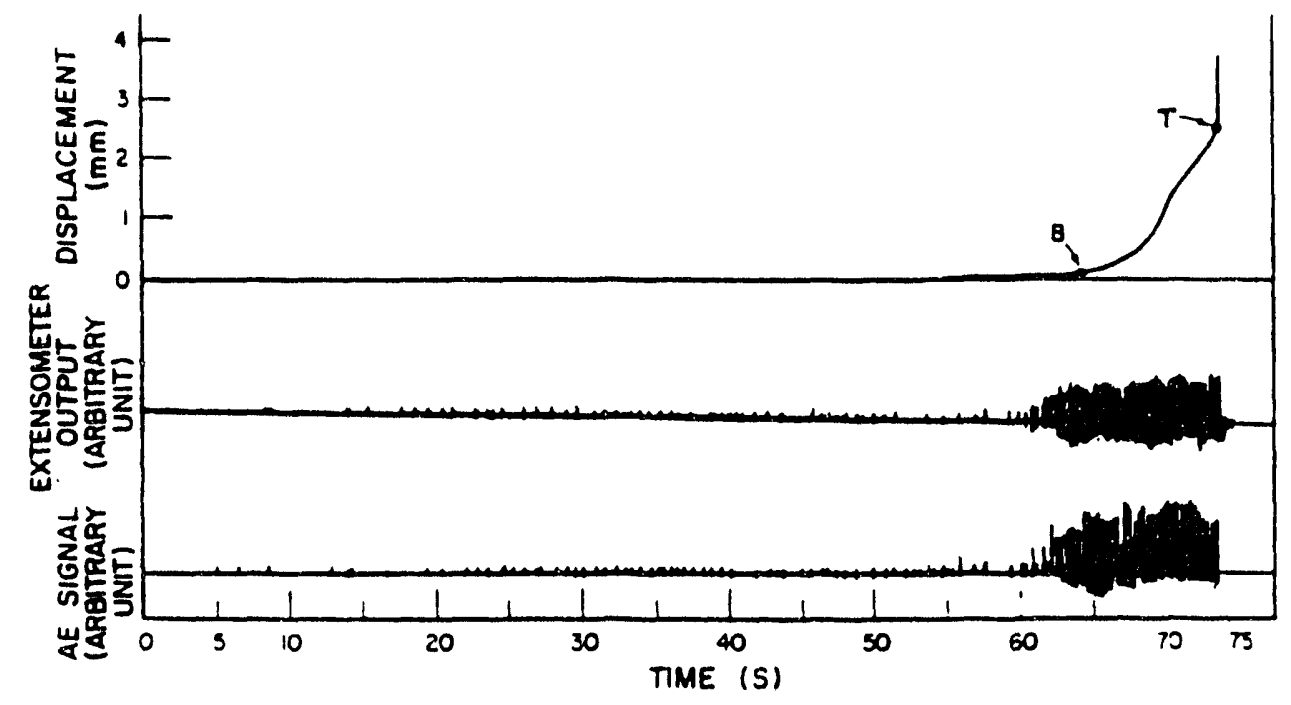

Figure 5.7: Correspondence between AE burst and magnetic extensometer displacement. Each small displacement is believed to result due to dicrontinuous motion comparable to the asperity dimersions. Figure from [12]. 
acteristic distance generally decreases with increasing specimen hardness. ${ }^{[12]}$ It is interesting to note that these microslip distances are comparable to the conductor displacements observed during quench experiments on high-performance winding models. $[5,6,12,13,15,17,18]$

It has long been recognized that sliding is not necessarily a steady process. As motion occurs the size, number, and relative strengths of the asperity junctions existing at any instant fluctuate due to the simultaneous formation and rupture of different asperity contacts. Because of their theoretical importance to contact and sliding phenomena several studies have been initiated to characterize the effect of load on the number and size distributions of asperity contacts between common surfaces with typical engineering surface roughnesses. Various methods have been tried, including: statistical analysis of the contacting surface roughness, ${ }^{[36,37]}$ direct visual observation of loaded transparent surfaces, ${ }^{[37,38]}$ electrical contact resistance measurements, ${ }^{[39]}$ and circumstantial evidence such as wear particle sizes ${ }^{[40]}$ and friction force variations. ${ }^{[23,41,42]}$ These methods all indicate that at light to moderate loads the average junction size rernains roughly constant; increasing the normal load produce a corresponding increase in the total number of contacts. The methods also suggest that the junction sizes generally follows a Gaussian distribution with about a factor of 10 spread between the largest and smallest contacts. Moderate loads are those for which the nominal, or apparent, contact pressure remains below $0.025 \sim 0.05$ of the softer surface's penetration hardness. As the normal load increases above these levels the real contact area assumes a significant fraction of the apparent area; this causes the junctions to grow together, thus reducing their numbers and increasing their average size.

Despite their similar conclusions these studies report widely divergent values of the contact sizes for apparently identical surfaces, depending on the measurement methods employed. Methods involving a direct observation ${ }^{[38]}$ or statical analysis of surface roughnesses ${ }^{[36]}$ indicate contact sizes in the $0.5-5 \mu \mathrm{m}$ range, while analyses based on more indirect measurements ${ }^{[37,39-42]}$ report junction sizes typically in the range $10-100 \mu \mathrm{m}$. Dyson and Hirst ${ }^{[38]}$ propose a partial resolution to these disparate values. Using phase contrast microscopy to directly observed contact areas, they noted that the smaller contacts are not uniformly distributed, rather 
they tend to cluster along the machining ridges formed during specimen preparation. Because of their close proximity these clustered contacts act cooperatively, with a range of interaction comparable with the average cluster diameter. This twotiered contact model is consistent with the transient sliding behavior for the nylon specimen (Figure 5.4a); the microslips correspond the disruption of the individual contact areas while the displacement prior to sliding reflects the range of cooperative action within the asperity clusters. Maeda, et al. ${ }^{[12]}$ observation that difference machining methods produce marked differences in the initial slip behavior of G-10 specimens is also suggestive of this two-tiered asperity contact model, given that each surface finishing technique is likely to produce markedly different asperity cluster groupings.

\subsection{Spring/electromagnetic-force/friction model}

Tsukamoto and Iwasa ${ }^{[8]}$ have developed a qualitative conductor-motion model based on the stylized winding geometry depicted in Figure 5.8. Figure 5.8 presents a cross-sectional view of a solenoidal winding in which an isolated conductor segment can move relative to the rest of the winding. The model assumes that the conductor is subjected to three main forces: spring, electromagnetic, and friction. The principal direction of motion is assumed to lie along the solenoid axis.

The radial force, $F_{r}$, can be decompused into: residual forces due to winding, thermal contraction forces, and radial electromagnetic forces. In a real solenoid, these radial forces vary both temporally and spatially, however, in this model, they are assumed constant for simplicity. Temporal variations in the radial force are produced by the time-varying electromagnetic forces during charging. Spatial variations are caused by statistical variations in the conductor thickness which alters the residual winding forces and by spatial variations in the radial electromagnetic forces.

The model assumes a constant, velocity-independent friction coefficient, $\mu_{s}$. The friction force, $F_{f}$, is bounded by the static friction force, $\mu_{s} F_{r}$.

$$
F_{f} \leq \mu_{s} F_{r}
$$

Static contact prevails when the net force on the conductor in less than the static friction force. 


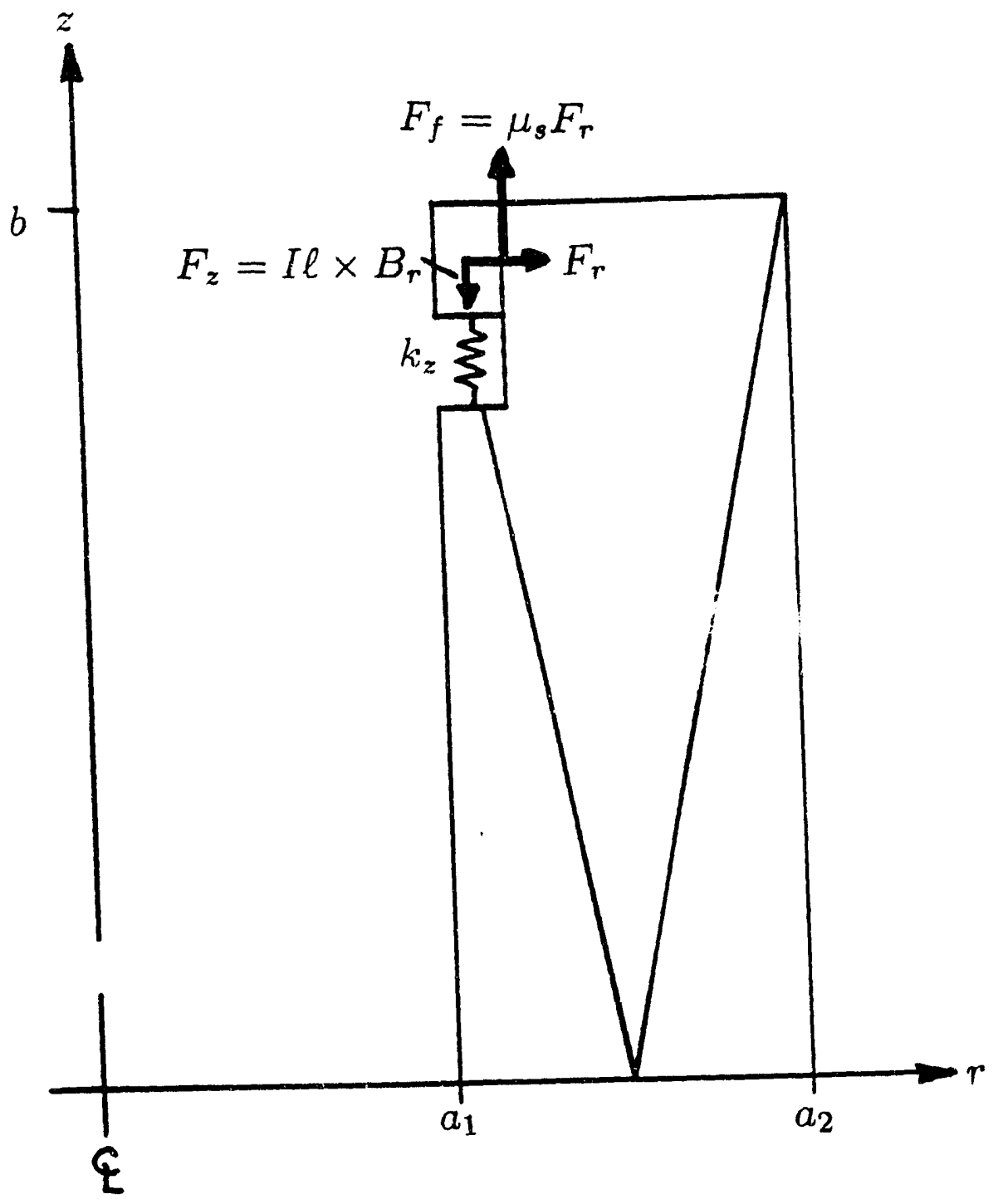

Figure 5.8: Schematic illustration of moving conductor segment in solenoidal winding. 
The axial force, $F_{z}$, is due entirely to electromagnetic forces.

$$
F_{z}=I \ell \times B_{r}
$$

where $I$ is the magnet current, $\ell$ is the length of moving conductor segment, and $B_{r}$ is the radial component of the local magnetic induction. The axial restoring force, $k_{z}$, is provided by the moving conductor segment acting as a leaf spring. The length of the moving conductor segment is determined by two factors. One is the variation in the radial contact forces along the conductor length and the second in fluctuations in the static friction force, for instance due to local surface contamination. Although the exact length of a moving conductor segment has not been measured in a real solenoid, its length has been estimated at about $\frac{1}{4}$ to $\frac{1}{2}$ of a turn. ${ }^{[18]}$

A necessary condition for motion is the presence of axial gaps between the conductors. Axial gaps can be caused by: 1) the inability to winding magnets to sub-micron tolerances, and 2) the axial thermal contraction of the conductors. Most solenoids are constructed with a predetermined radial precompression, but virtually no axial stress. As the magnet is cooled the center of each conductor remains essentially fixed in its "as-wound" position while the edges of the conductors contract away from each ather. The widths of the conductors used in high-performance windings typically range from 1 to $5 \mathrm{~mm}$. For a thermal contraction ratio of $\sim 3 \times 10^{-3}$ this leaves and interconductor spacing at $4.2 \mathrm{~K}$ of $3-15 \mu \mathrm{m}$, well within the range of slip distances reported during conductor-motion quench experiments.

Figure 5.9 presents the basic concept behind the conductor-motion force analysis. The force balance for a moving conductor segment is:

$$
F_{f}=F_{z}-k_{z} z
$$

The first charging sequence starts at point $\mathbf{A}$ on the force displacement diagram. The net force increases with increasing current, however, no motion occurs until the static friction force is exceeded at point $\mathbf{B}$. Motion above point $\mathbf{B}$ occurs in a stepwise fashion; each step corresponds to a microslip. For simplicity, the fine-scale details of these microslips were not included in Figure 5.9. The charging sequence is completed without a quench at point $\mathbf{D}$. 


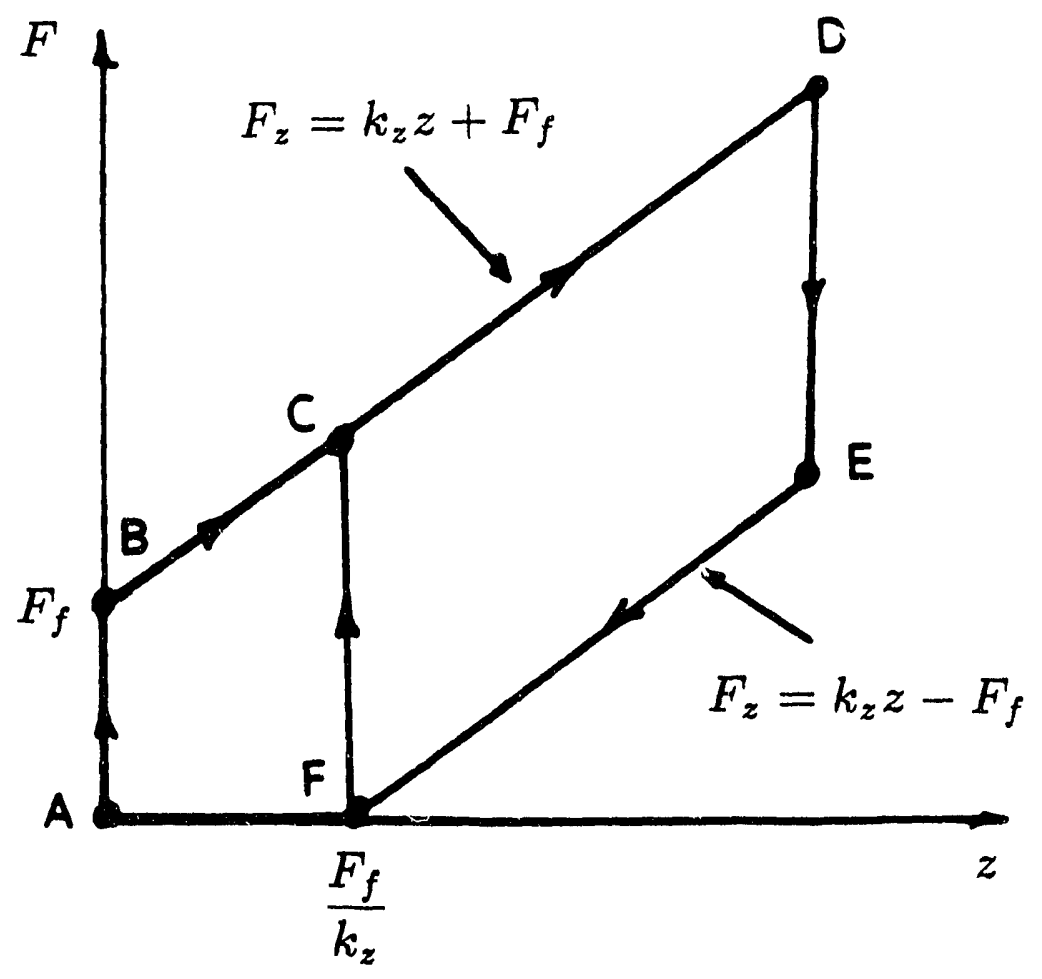

Figure 5.9: Spring/electromagnetic-force/friction force model, used to interpret reversibility of conductor motion. The friction force intercept, $F_{f}$, refers to the static friction force, $F_{f}=\mu_{s} F_{r}$. Figure from [8]. 
As the current decreases, the friction force is reversed. The conductor remains stationary until point $\mathbf{E}$ where the static friction force is again exceeded in the reverse direction. Microslips are again observed below point $\mathbf{E}$ and persist until $\mathbf{F}$ at which point the first charging sequence is complete. During subsequent charging sequences the force-displacement trajectory follows the path FCDEF, with repeatable sliding characteristics. A permanent offset in the segments equilibrium position, from $\mathbf{A}$ to $\mathbf{F}$, occurs during the first charging sequence.

The general validity of this conductor motion model was confirmed using a short, non-inductive, single-layer solenoid. Because a non-inductive winding produces no net field of its own, this coil was placed in the bore of a second, slightly larger superconducting solenoid which provided a constant background radial magnetic field. Figure 5.10 presents the results of the cumulative acoustic emissions $v s$ charging curient during these tests. The dashed line in the figure is an analytical curve fit of the data, derived from this conductor motion model; the exact details of this derivation are provided in [8]. The experimental results are consistent with the model in several regards.

1. As the current is increased from zero, acoustic emissions are observed only above a certain minimum current, when the electromagnetic force exceeds the static friction force.

2. A portion of the initial sliding sequence is irreversible; this corresponds the the shift from $\mathbf{A}$ to $\mathbf{F}$ in the segments equilibrium position (Figure 5.9). During subsequent charging sequences the minimum current needed for motion increases while the cumulative $\mathrm{AE}$ is lower.

3. The AE vs current trace (Figure 5.10) is reproducible in all but the first charging sequence. 


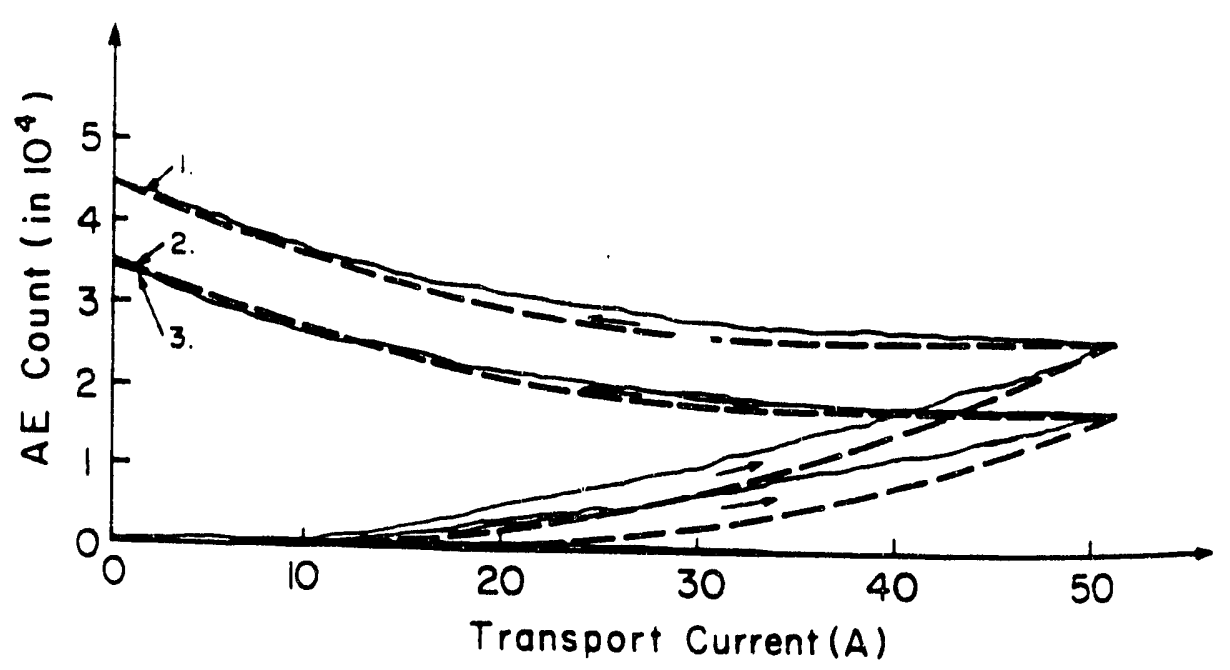

Figure 5.10: Results of conductor motion experiments on single layer solenoidal winding showing cumulative $A E$ versus current. The dashed line plots theoretical curve fit on the data based on the based on the analysis derived in [8]. Figure from [8]. 


\subsection{Conductor motions in high-performance solenoids}

\subsubsection{Motivation}

Conductor motion research directed towards high-performance magnets is fundamentally different from that directed towards cryostable magnets. In cryostable magnets the degree of cryogenic cooling can be treated as a major variable; research is directed towards estimating the magnitude of potential disturbances and providing just enough cooling to stabilize the magnet against these disturbances. By contrast, the absence of cooling passages in high-performance magnets sets a maximum permissible disturbance energy; the magnet's corresponding minimum quench energy was discussed in $\mathrm{Ch}$. 3. Thus, successful high-performance windings must be constructed so that no disturbance exceeds this quench energy threshold. Consequently, the background information needed to evaluate conductor-motion disturbances has evolved much more gradually, because these disturbances fall under a much more restrictive set of quench energy guidelines.

The relation between a high-performance solenoid's construction method and its training behavior was first examined during he late 1960 s by the research group at the Rutherford Laboratory, shortly after the development of composite superconductors. ${ }^{[43]}$ This study reported severe-to-moderate degradation in their dry-wound solenoids which they attributed to sudden wire motions of greater than $\sim 3 \mu \mathrm{m}$ displacement. The study also noted markedly different training behaviors for apparently identical solenoicis constructed at different times by different technicians. By contrast, their impregnated windings demonstrated virtually degradationfree performance; based on these results they asserted that the complete elimination of wire motion is necessary for reliable high-field magnet operation. During the past twenty years high-performance research had been devoted almost entirely to the perfection of the impregnated winding technique. However, as $§ 3.2 .2 .2$. indicates, the higher operating limits demanded of these magnets has since required increasingly complex and more costly manufacturing techniques to minimize the occurrence of impregnant-cracking disturbances. Considerable interest has recently been been focussed on reexamining dry-wound magnets with the intent of developing less costly manufacturing methods. 


\subsubsection{Probable quench initiation locations}

A key feature of the recent investigations of conductor-motion-induced quenches is the identification of the most probable quench in:iation locations. Working predominantly with single-lavered winding models. Chikaba, et al. ${ }^{[15]}$ determined that the principal direction of motion does indeed lie along the solenoid axis. Using a combination of strain gauge and $\mathrm{AE}$ measurements they showed that:

1. The tensile circumferential strain varies smoothly and reversible during all charging/discharge cycles.

2. The axial strain varies discontinuously during the first few charging cycles. These discontinuities result from abrupt conductor motions in the vicinity of the strain gauge. The axial strain measurements indicate that conductor segments near the winding's ends are displaced towards the winding midplane; this displacement is consistent with the large axial electromagnetic forces that act on these conductor segments.

3. The AE activity and axial strain depenc on the charging sequence. As a winding trains, its average $\mathrm{AE}$ energy decreases and the axial strain vs current trace becomes largely repeatable. The persistent occurrence of $\mathrm{AE}$ and hysteresis in the axial strain measurements indicate that repeatable wire motions can exist in a well trained magnet, so long as the energy dissipation remains below the quench energy; this condition is relatively easy to achieve in a single layer winding models where roughly half of the conductor's surface area is exposed to liquid helium.

Urata and Maeda, ${ }^{[17]}$ on the other hand, examined the radial distribution of conductor-motion-induced quenches in full-scale superconducting solenoids. They used the $\mathrm{AE} /$ voltage method to examine the quench behavior of a 24-layer winding. Voltage taps were soldered to the ends of each winding layer to determine the quench-initiation layer. Figure 5.11 presents the results of these experiment. Figure 5.11 shows the maximum current obtained during the magnet's first twenty charging cycles and demonstrates two effects. The first is that the magnet trains slowly, reaching its critical current (at the winding's peak field of $8.1 \mathrm{~T}$ ) after 19 charging cycles. The second is that all but one of the quenches started at the two innermost 
winding layers.

The preponderance of inner layer quenches is consistent with the quench energy distribution in the winding. Figure 3.1 illustrates the dependence between the quench energy density, normalized charging current, and local magnetic induction. For a uniform current density solenoid, the minimum quench energy occurs at the winding's inner diameter where both the local magnetic induction and fielddependent normalized current are at their maximums.

\subsubsection{Conductor-motion stabilization}

Several methods have been suggested for preventing conductor-motion-induced quenches. The most frequently proposed solution is to eliminate disturbances by preventing conductor motions altogether; in dry windings this effect is attempted by winding the magnet so tightly that the conductors' nominal static friction forces exceed their axial electromagnetic forces. An alternate approach to preventing quenches is to reduce the maximum disturbance energy to below the winding's local quench energy. For impregnated solenoids this effect is achieved by allowing the winding to radially separate from its coil form during energization; this radial separation greatly reduces the magnitude of the shear stresses induced at the inner radius near the coil's ends. ${ }^{[44]}$

Urata and Maeda ${ }^{[18]}$ have constructed full-scale dry-wound solenoids to examine both of these stabilization techniques. Figure 5.12 shows both the training and disturbance histories for a 24-layer solenoid wound with a 150-MPa conductor tension. For a conductor static friction coefficient of 0.26 , this level of winding tension was determined to provide sufficient radial precompression to prevent conductor motion even at full current operation. The conductor was cold worked prior to winding so that these high stress levels would remain within the conductor's elastic !imit. Figure 5.13 shows the training and disturbance histories for a similar 24-layer solenoid, this time would with a $30-\mathrm{MPa}$ conductor tension. This magnet separates from its coil form as its charging current approaches about half of its critical current.

Figures 5.12 and 5.13 show that the quench and training histories are markedly different for the two coils. For the tightly wound solenoid (Figure 5.12a), the first quench occurs at $84 \%$ of the critical current, however, the magnet trains slowly and 


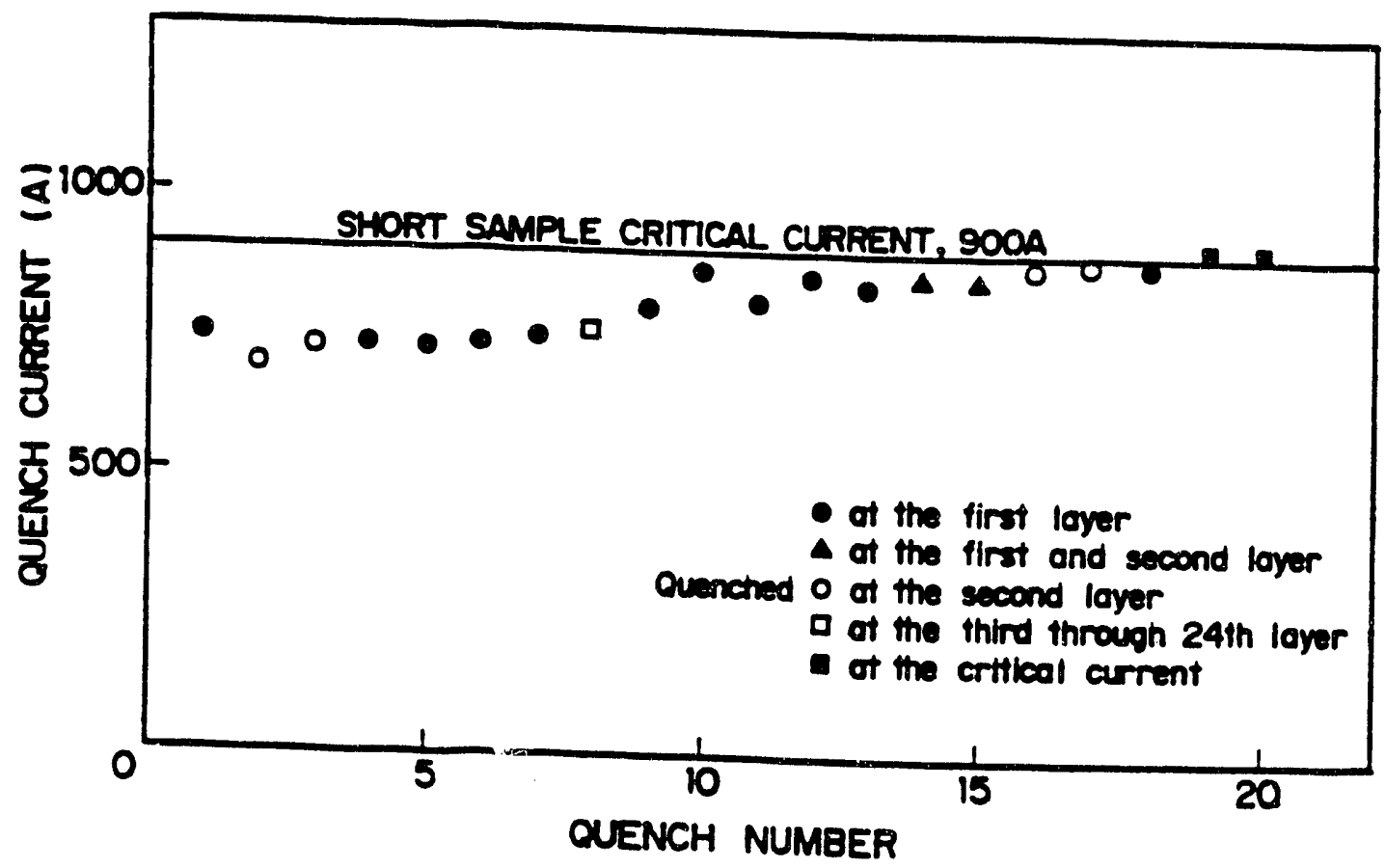

Figure 5.11: Results of quench-current experiments demonstrating preponderance of conductor-motion-induced quenches occur near windings inner diameter. Figure from $[17]$. 
demonstrates degraded behavior with a steady-state quench current of about $95 \%$ of the conductor's critical current. By contrast, the more loosely wound coil (Figure $5.13 \mathrm{a})$, initially quenches at $67 \%$ of its critical current, however, it trains rapidly and achieves full current performance after nine training sequences.

Large differences are also observed in the disturbance histories obtained during the coils' training sequences (Figures $5.12 \mathrm{~b}$ and $5.13 \mathrm{~b}$ ). Each voltage spike in these traces corresponds to a microslip; the height of the voltage signals is roughly proportional to the slip distance. The results for the tightly wound coil (Figure 5.12b) indicates that the high winding tension is only partially effective in preventing wire motion. The absence of large voltage spikes in Figure $5.12 \mathrm{~b}$ indicates that high winding tension does suppress large wire motions; however, the figure also reveals the persistent occurrence of several small microslips, many of which recur during subsequent charging cycles. On the other hand, the more loosely wound coil (Figure $5.13 \mathrm{~b}$ ) shows a very pronounced Kaiser effect; during subsequent charging cycles, conductor motions are not observed until the charging current exceeds the quench current obtained during the previous training cycle.

The training behaviors of the two coils can be qualitatively interpreted using the conductor-motion model discussed in $§ 5.2$. Because each solenoid consists of numerous conductor segments, each of which is capable of independent motion, it should be realized that the hypothetical force-displacement diagram for an entire magnet is at best a multidimensional aggregate over all of the conductor segments. The following description is directed only at those segments which actually move during a training cycle. During the loosely wound coil's initial training sequences the winding follows a path similar to the ABCFA friction-force/displacement trajectory in Figure 5.9; during training the conductors shift towards more stable equilibrium positions without experiencing any overshoot. The disturbances in Figure 5.13b occur after the magnet has separated from its coil form, hence, the progressive improvement in its performance most probably results through the elimination of interconductor spaces. By contrast, the elimination of interconductor spaces in the tightly-wound solenoid is prevented by the winding's designed intent of suppressing conductor motions altogether. The aggregate force-displacement behavior for the tightly-wound solenoid consists not only of irreversible ABCFA trajectories 

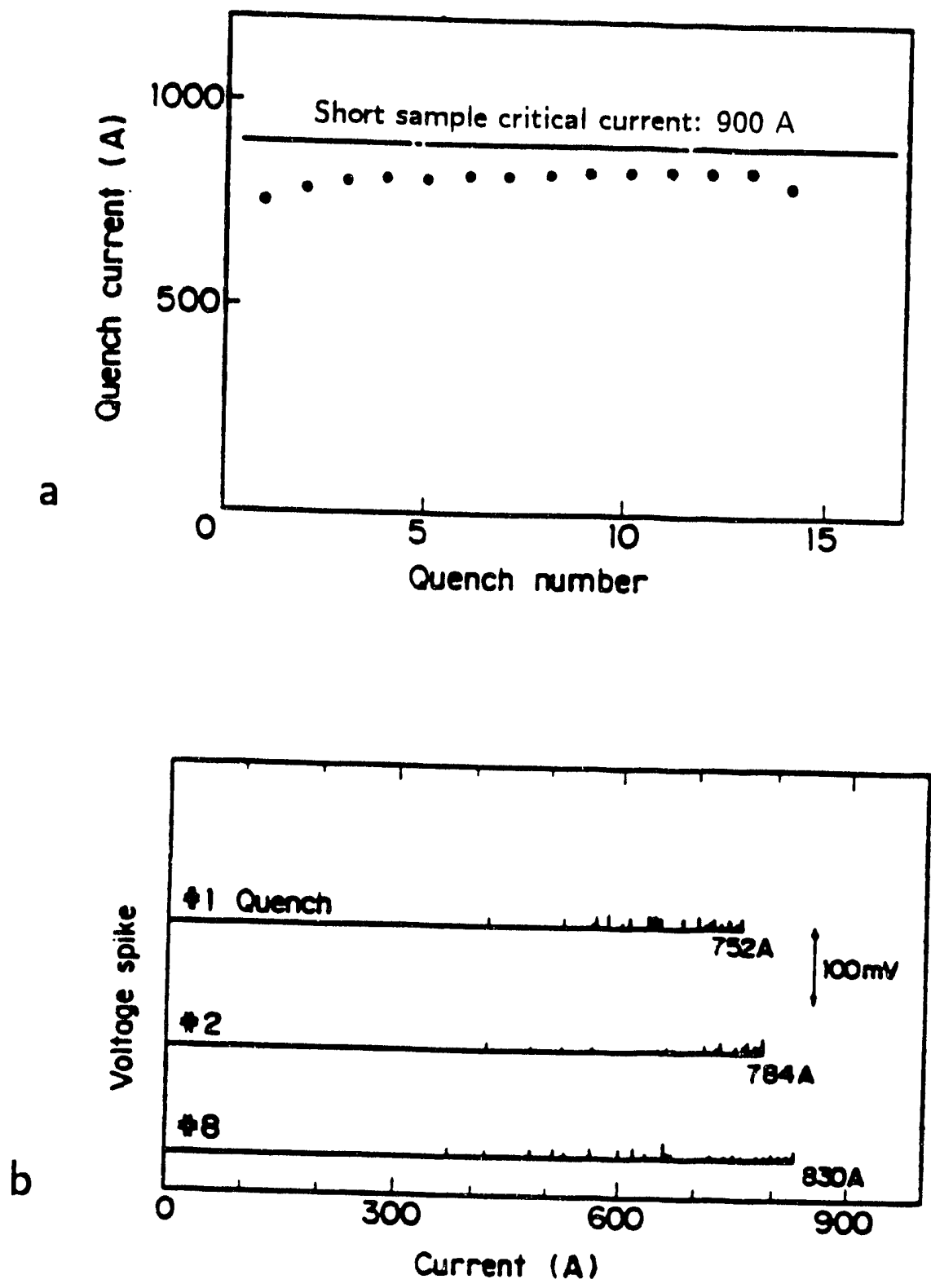

Figure 5.12: a) Quench, and b) disturbance histories for a 24-layer solenoid wound with 150-MPa conductor tension. Figures from [18]. 

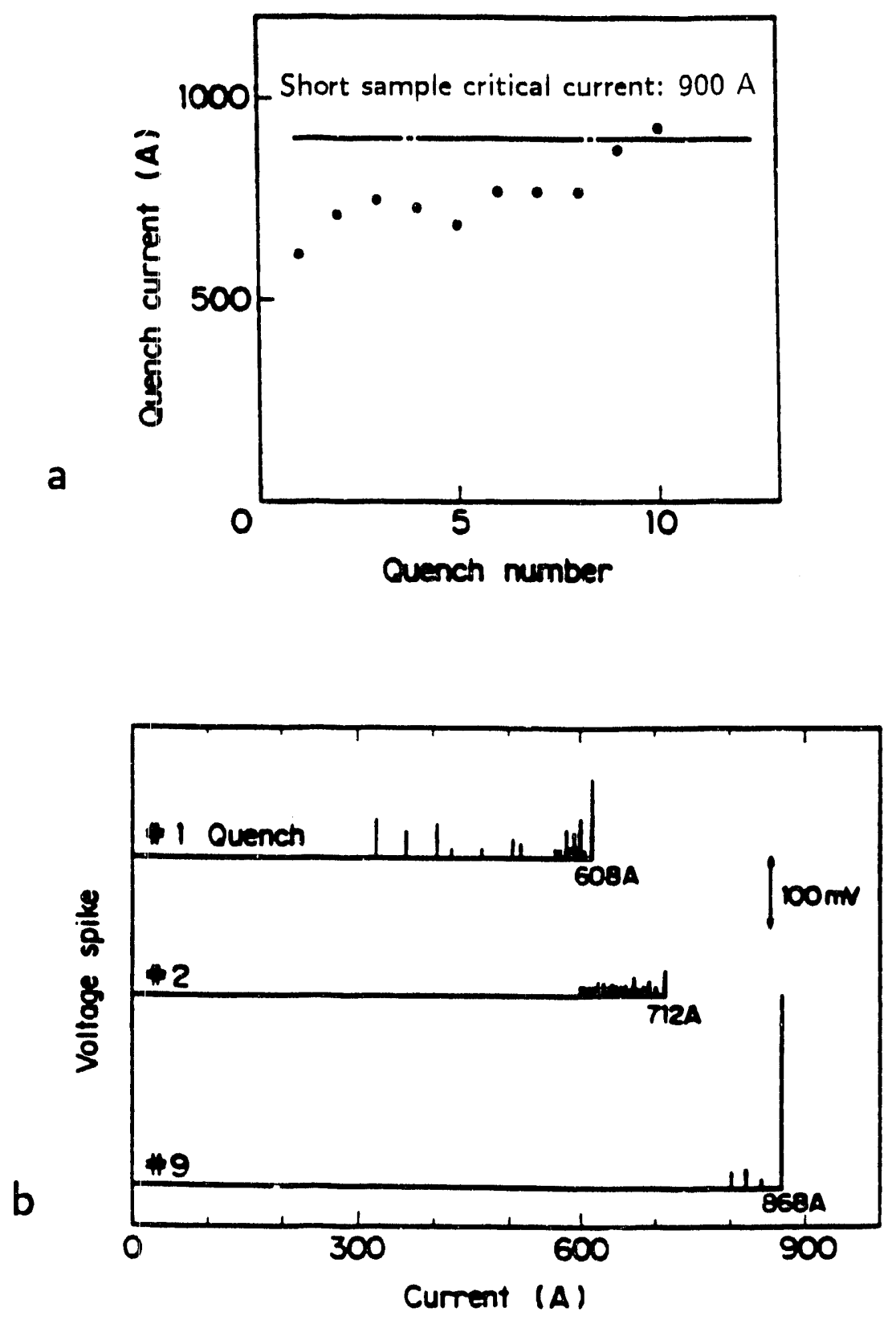

Figure 5.13: a) Quench, and b) disturbance histories for a 24-layer solenoid wound with 30-MPa conductor tension. Figures from [18]. 
but also of reversible FCDEF trajectories. After several charging cycles only the FCDEF components of this trajectory remain. The degraded performance persists because the energy dissipated durirg the friction-force/displacement trajectory exceeds the magnet's minimum quench energy.

\subsubsection{Friction of dry-winding constituents}

Little has been done to characterize the frictional properties of the conductors commonly used in dry-wound high-performance solenoids. These conductors are usually insulated with several thin coating of Formvar, a cross-linked polyvinylformal-based enamel. From a construction standpoint, Formvar has several desirable properties. It is viscoelastic at room temperature, this gives it good durability, high abrasion resistance, and the ability to withstand rough treatment during manufacture. Unlike most other organic insulations, it does not delaminate from the conductor upon cooling to $4.2 \mathrm{~K}$. The coating thickness depends on the overall conductor dimensions but is typically in the range $25 \sim 75 \mu \mathrm{m}$. This insulation prevents dielectric breakdown between the conductors during quench and allows the magnet to be very densely constructed since no further electrical insulation or structural support is needed.

Urata and Maeda ${ }^{[18]}$ have examined sliding initiation between Formvar insulated superconductor pairs using a linear friction apparatus similar to that discussed in §5.1.4.1. Figure 5.14 shows an example of one of their Formvar/Formvar forcedisplacement diagrams. During the initial portion of the loading cycle they observed several microslips of 3-5 $\mu \mathrm{m}$ displacement; however, above a friction coefficient of 0.26 the slip distance increase to $50 \sim 100 \mu \mathrm{m}$ displacements.

\subsection{Summary}

All of the cryogenic-temperature sliding pairs examined to date exhibit discontinuous motion on a microscopic scale; these discontinuities are manifest in the microslips discussed in $\$ 4.1 .4 .4$. The relative displacement during a microslip is typically in the range $1 \sim 3 \mu \mathrm{m}$. Both theoretical analysis and experimental measurements (§3.3.3.) indicate that the energy dissipated during these abrupt microslips is sufficient to initiate quenching in a high-performance solenoid operating near its critical surface. The remainder of the thesis will investigate various approaches for 


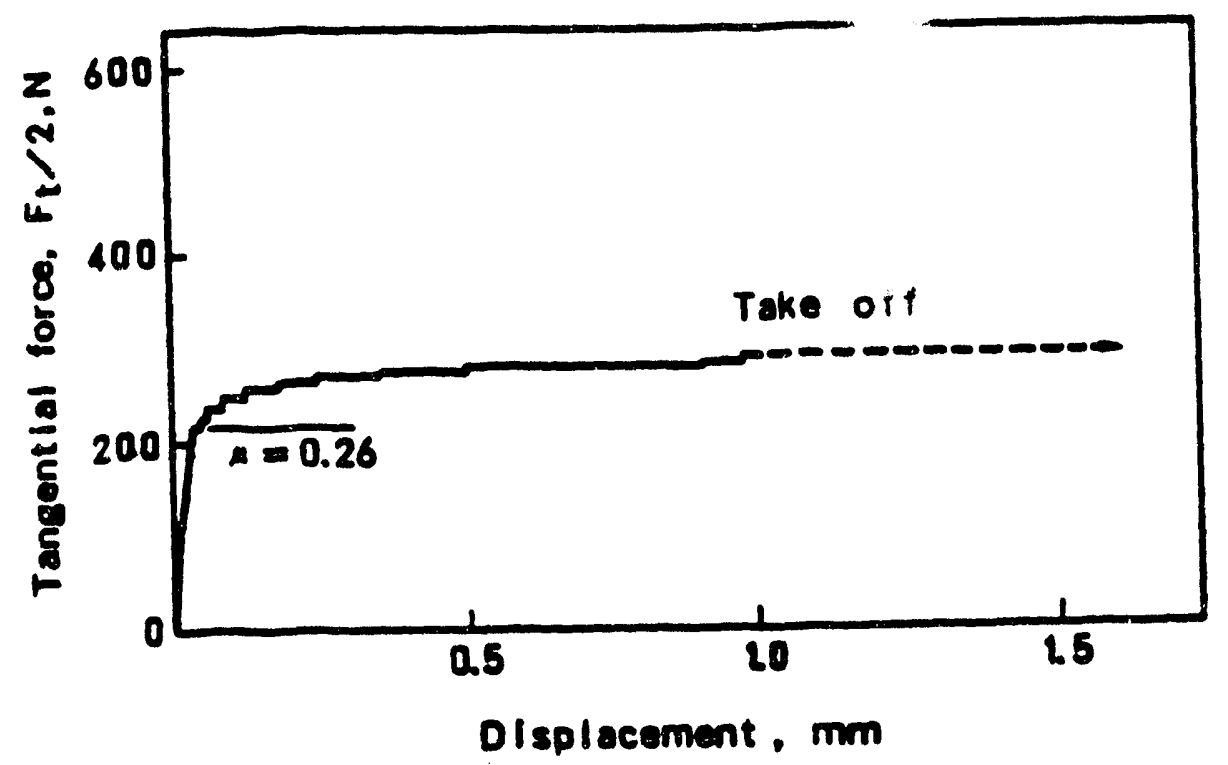

Figure 5.14: Friction force/displacement for a sliding pair tsisting of two Formvarinsulated composite superconductors. Figure from [18]. 
minimizing microslip-induced quenching.

Chapter 6 will present a preliminary assessment of the prospects for significantly altering $4.2-\mathrm{K}$ microslip behavior. The creep theory of friction ${ }^{[24]}$ suggests the existence of a class of materials whose asperity junction population during sliding remains essentially constant. The theory contends that a sliding interface provides a unique geometry from the standpoint of steady-state creep testing, that is, the test can proceed indefinitely without the possibility of rupture; material is continually added to the leading edge of each junction to compensate for that lost on the trailing edge. Continuous displacement at the asperities level should eliminate abrupt microslips and thus greatly reduce the microslip-induced heating.

Chapter 7 extends the structural approach discussed in $§ 5.3 .3$. to very low stress levels. Based on the available experimental evidence, we conclude that conductor motions during the first few charging cycles are inevitable. Hence, the intent of the approach directed not at eliminating conductor motions but at allowing these motions to occur near the start of energization, where the magnet's quench energy density is relatively high. If a winding's incipient conductor motions can be induced to occur at lower operating currents its relative stability should proportionally increase. 


\section{References for Chapter 5}

1. Y. Iwasa, R. Kensley, and J.E.C. Williams, Frictional properties of metalinsulator interfaces, IEEE Trans. MAG-15 (1979) 36-39.

2. Kensley, R.S., and Iwasa, Y. Frictional properties of metal insulator surfaces at cryogenic temperatures Cryogenics 20 (1980) 25-35.

3. Kensley, R.S., Maeda, H., and Iwasa, Y. Transient slip behaviour of metal/insulator pairs at 4.2 K Cryogenics 21 (1981) 479-489.

4. Kensley, R.S., Maeda, H., and Iwasa, Y. Frictional disturbances in superconducting magnets IEEE Trans. MAG-17 (1981) 1068-1071.

5. Tsukamoto, O., Maguire, J.F., Bobrov, E.S., and Iwasa, Y. Identification of quench origins in a superconductor with acoustic emission and voltage measurements Appl. Phys. Lett. 39 (1981) 172-174.

6. Tsukamoto, O., Maeda, H., and Iwasa, Y. Microslip-induced degradation in a braided superconductor Appl. Phys. Lett. 39 (1981) 918-920.

7. O. Tsukamoto, M.W. Sinclair, M.F. Steinhoff, and Y. Iwasa, Origins of acoustic emissions in superconducting wires, Appl. Phys. Lett. 38 (1981) 718-720.

8. Tsukamoto, O., and Iwasa, Y. Sources of acoustic emission in superconducting magnets J. Appl. Phys. 54 (1983) 997-1007.

9. Tsukamoto, O., Takao, T., and Honjoh, S. Estimating the size of disturbances due to conductor motion in superconducting windings IEEE Trans. MAG-24 (1988) 1182-1185.

10. Tsukamoto, O., Takao, T., Honjo, S. Quantification of disturbance energy due to conductor motion and stability analysis of superconducting composite wire IEEE Trans. 25 (1989) 1713-1716.

11. Takao, T., Honjo, S., and Tsukamoto, $O$. Technique to reduce disturbance energy due to frictional conductor motion in superconducting magnet Proc. 11th Int. Conf. Magnet Tech. MT-11 Japan Institute of Electrical Engineers (1990) 1108-1113.

12. H. Maeda, O. Tsukamoto, and Y. Iwasa, The mechanisms of frictional motion and its effects at $4.2 \mathrm{~K}$ in superconducting magnet winding models, Cryogenics 22 (1982) 287-295.

13. Maeda, H. Mechanical disturbances for a cable-in-substructure superconductor Cryogenics 24 (1984) 208-210.

14. Takao, T., and Tsukamoto, $O$. Stability against the frictional motion of conductor in superconducting windings IEEE Trans. MAG-27 (1991) 2147-2150.

15. Chikaba, J., Irie, F., Funaki, K., Takeo, M., and Yamafuji, K. Instabilities 
due to mechanical strain energy in superconducting magnets IEEE Trans. MAG23 (1987) 1600-1603.

16. Chikaba, J., Irie, F., Takeo, M., Funaki, K., and Yamafuji, K. Relation between instabilities and wire motion in superconducting magnets Cryogenics 30 (1990) 649-653.

17. Urata, M., and Maeda, H. Stabilization of superconducting dry solenoids IEEE Trans. MAG-25 (1989) 1528-1531.

18. Urata, M., and Maeda, H. Relation between radial stress and quench current for tightly wound dry solenoids IEEE Trans. MAG-23 (1987) 1596-1599.

19. Wilson, M.N., and Iwasa, Y. Stability of superconductors against localized disturbances of limited magnitude Cryogenics 18 (1978) 17-25.

20. Stekly, Z.J.J, and Zar, J.L. Stable superconducting coils IEEE Trans. NS-12 (1965) 367-372.

21. Martinelli, A.P. and Wipf, S.L. Investigation of cryogenic stability and reliability of operation of $\mathrm{Nb}_{3} \mathrm{Sn}$ coils in helium gas environment Proc. 1972 Appl. Supercond. Conf. IEEE Pub. 72ch0682-5-TABSC (1972) 331-340.

22. Iwasa, Y. Stability and protection issues in high-performance superconducting magnets Cryogenics 31 (1991) 575-579.

23. Rabinowicz, E. The nature of the static and kinetic coefficients of friction $J$. Appl. Phys. 22 (1951) 1373-1379.

24. Burwell, J.T., and Rabinowicz, E. The nature of the coefficient of friction $J$. Appl. Phys. 24 (1953) 136-139.

25. Dokos, S.J. Sliding friction under extreme pressures J. Appl. Mech. A13 (1946) 148-156.

26. Michael, P.C., Aized, D., Rabinowicz, E., and Iwasa, Y. Mechanical properties and static friction behavior of epoxy mixes at room temperature and at $77 \mathrm{~K}$ Cryogenics 30 (1990) 775-786.

27. Rabinowicz, E. A study of the stick-slip process Friction and Wear: Proc. Symp. on Friction and Wear, Detroit - 1957 (ed. R. Davies) Elsevier, New York (1959) 149-164.

28. Iwasa, Y., and Rabinowicz, E. Cryotribology (Low-temperature friction and wear): Development of cryotribological theories and application to cryogenic devices Renewal proposal to the U.S. Dept. of Energy Office of Basic Energy Sciences, Washington, DC (1991).

29. Ohara, T., Umeda, M., Agatsuma, K., Kosaka, S., Iwasa, Y., and Takeuchi, A. Cryogenic creep measurements system using laser light interference $C E C / I C M C$ conference (1989). 
30. Briggs, J.C. Force identification using extracted parameters with applications to glide height testing of computer hard disks PhD. thesis, MIT (1991) pp. 22-25.

31. Iwasa, Y. Acoustic emission diagnostic techniques for high-performance superconducting dipoles, Renewal proposal submitted to the U.S. DOE, High Energy Physics Div. (1987) p11-15.

32. Belyi, V.A., Kholodilov, O.V., and Sviridyonok, A.I. Acoustic spectrometry as used for the evaluation of tribological systems Wear 69 (1981) 309-319.

33. Tse, M., and Lewis, A.F. Triboacoustics of nonwoven fabric/floppy disk dynamic contact Tribol. Mech. Magn. Stor. Sys., III ASLE SP-21 (1986) 63-71.

34. Benson, R.C., Sundaram, R., and Talke, F.E. A study of acoustic emission from the slider/disk interface in a $5 \frac{1}{4}$ inch hard disk drive Tribol. Mech. Magn. Stor. Sys., V STLE SP-25 (1988) 87-93.

35. Bhushan, B. Tribology and mechanics of magnetic storage systems, SpringerVerlag, New York (1990) pp. 143-145,571-573.

36. Gupta, P.K., and Cook, N.H. Statistical analysis of mechanical interaction of rough surfaces $J$. Lub. Tech. (1972) 19-26.

37. Uppal, A.h., Probert, S.D., and Thomas, T.R. The real area of contact between a rough and a flat surface Wear 22 (1972) 163-183.

38. Dyson, J., and Hirst, W. The true contact area between solids Proc. Phys, Soc. London 67 (1954) 309-312.

39. Holm, R. Electric Contacts, Theory and Application Springer-Verlag, New York (1967).

40. Rabinowicz, E. The dependence of the adhesive wear coefficient on the surface energy of adhesion Wear of Materials - 1977 ASME, New York (1977) 36-40.

41. Rabinowicz, E. Autocorrelation analysis of the sliding process J. Appl. Phys. 27 (1956) 131-135.

42. Courtney-Pratt, C.S., and Eisner, E. The effect of a tangential force on the contact of metallic bodies Proc. Roy. Soc. A 238 (1957) 529-550.

43. Smith, P.F., Wilson, M.N., and Spurway, A.H. Filamentary superconducting composites - IV. DC coil tests J. of Phys.; D - Appl. Phys. 3 (1970) 1561-1573.

44. E.S. Bobrov, J.E.C. Williams, and Y. Iwasa, Experimental and theoretical investigation of mechanical disturbances in epoxy-impregnated superconducting coils. 2. Shear-stress-induced epoxy fracture as the principal source of premature quenches and training - theoretical analysis, Cryogenics 25 (1985) 307-316. 


\section{Cryogenic sliding stabilization}

This chapter presents a more traditional tribological perspective for examining cryogenic-temperature sliding stability. An abbreviated mathematical model for stick-slip is developed. This stick-slip model is then used to qualitatively highlight two common methods for moderating frictional instabilities.

The first method is to select sliding components based on their inherently favorable frictional properties. The adhesion friction theory proposes several materials for room-temperature use, based on their bulk physical properties. The adhesion theory was shown in Ch. 4 to remain valid at cryogenic temperatures after accounting for the sliding materials' low-temperature strength properties. The chapter discusses the physical bases for room-temperature sliding stability and examines the material limitations that restrict the cryogenic-temperature applicability of contemporary material selection guidelines.

A second approach to stick-slip stabilization is to alter the sliding system's structural dynamics. If the potential slip distance can be reduced to below the average junction diameter, unstable conductor motions can be largely eliminated Structural dynamic models are developed for various conductor segment lengths. These models are incorporated into a magnet design implemented in Ch. 7 to investigate this structural approach for magnet stabilization.

\subsection{Stick-slip instabilities}

When a body is slid over another under a steady pulling force it is frequently observed that the motion proceeds sometimes at a constant or near-constant velocity and on other occasions at velocities that fluctuate widely. The oscillatory type of motion is generally referred to as "stick-slip". During the stick portion of the cycle the sliding surfaces remain in static contact for a time before breaking free and slipping relative to each other. All stick-slip processes are caused by the fact that the friction coefficient does not remain constant as a function of some other variable. This variable can be distance, velocity, time, or temperature. ${ }^{[1,2]}$ 
In most practical sliding systems stick-slip is considered a nuisance; in superconducting magnets it is an anathema. This is especially true in high-performance magnets where the conductor slippage is a side-effect of the dry-wound construction technique rather than an intended function of the magnet. Both theoretical prediction (Ch. 3) and experimental evidence (Ch. 5) indicate that conductor motion stick-slips amounting to $\sim 5 \mu \mathrm{m}$ total displacement are sufficient to prematurely quench the superconducting winding. If stable magnet operation is to be achieved some means must be devised either for eliminating stick-slips entirely, or for reducing the energy dissipation during slip to a manageable level.

\subsubsection{Stick-slip model}

Figure 6.1 presents the dynamic, lumped-parameters mass-spring-friction model commonly employed in the tribological literature for analyzing stick-slip instabilities. The model consists of a slider of mass $m$ that is pressed against its countersurface by its own weight, $m g$. The slider is connected to a constant velocity source $z_{a}(t)=v_{0} t$ by a spring, $k$, that represents the sliding system stiffness. The friction force between the slider and countersurface is denoted by $\mu(z, \dot{z}) m g$. The friction coefficient, $\mu(z, \dot{z})$, assumed in this model varies both with displacement and velocity. The specific form of the friction coefficient variation will be discussed in $\S 6.1 .3 .1$.

This displacement-based model is most frequently used because it is relatively easy to verify experimentally. Fortunately, conclusions regarding a sliding system's dynamic stability are unaffected by the decision to pursue this velocity-based model, rather than the force-based conductor-motion model proposed in $\$ 5.2$.

\subsubsection{Deformation during the stick portion of the stick-slip cycle.}

The tribological literature recognizes that substantial deformation occurs on the asperity scale during sliding initiation. ${ }^{[3-8]}$ It will be helpful to review the extent of asperity deformation prior to sliding initiation before deriving the dynamic system equation corresponding to Fig. 6.1. One of the most detailed experimental determination of this pre-sliding displacement was performed at room temperature by Courtney-Pratt and Eisner using multiple-beam optical interferometry. ${ }^{\text {[3] }}$

The general arrangement of Courtney-Pratt and Eisner's test rig corresponds 
to the model depicted in Figure 6.1. The tangential force applied to the slider was regulated by the displacement $z_{a}(t)$ imposed at the free end of the elastic loading element $k$. The tangential force during these experiments was applied incrementally. During each load increment the relative displacement, $z$, of the slider was measured to the nearest $1 \mathrm{~nm}$ using a multiple beam interferometer. One half of the interferometer was mounted to the slider while the other was rigidly fixed to the counterface as near to the slider as possible. Because the interference fringes were counted manually a slow loading rate was used. The combination of a displacement-controlled tangential force coupled with a slow, incremental loading rate precludes the development of long-range frictional instabilities; as the slider moves, the applied friction force gradually decreases.

Typical results from Courtney-Pratt and Eisner's experiments for a platinumon-platinum sliding pair are reproduced in Figure 6.2. The results show the total displacement following each load increment. The open symbols in Figure 6.2 are for unlubricated surfaces while the filled symbols represent surfaces lubricated by a solution of lauric acid in cetane. The lubrication reduces the effective adhesion between the sliding surfaces thus reducing the static friction coefficient, however, the general shape of the force-displacement curve remains unchanged.

The relative displacement between the two surfaces is a smooth increasing function of the applied force; any applied friction force less than the static value produces a certain equilibrium displacement. As the applied friction force approaches the static friction force this displacement increased more and more rapidly leading ultimately to continuous sliding. The asymptotic approach towards the static friction force typically occurred within a displacement of $1 \sim 10 \mu \mathrm{m}$. ${ }^{[3]}$ Similar results were observed with several other metal-metal pairs. The transition from static contact to sliding was also measured by Rabinowicz, using an impact-loading method. ${ }^{[7]} \mathrm{His}$ measurements also show that the static friction coefficient persists for a preliminary displacement in the $1 \sim 10 \mu \mathrm{m}$ range. These displacements are of the same order of magnitude as the typical asperity junction diameter and are approximately the same as the unstable slip distances observed in high-performance magnets.

For analytical purposes this pre-sliding deformation is modeled as an interfacial junction stiffness. Using $z^{*}$ to denote the transition from static contact to sliding, 


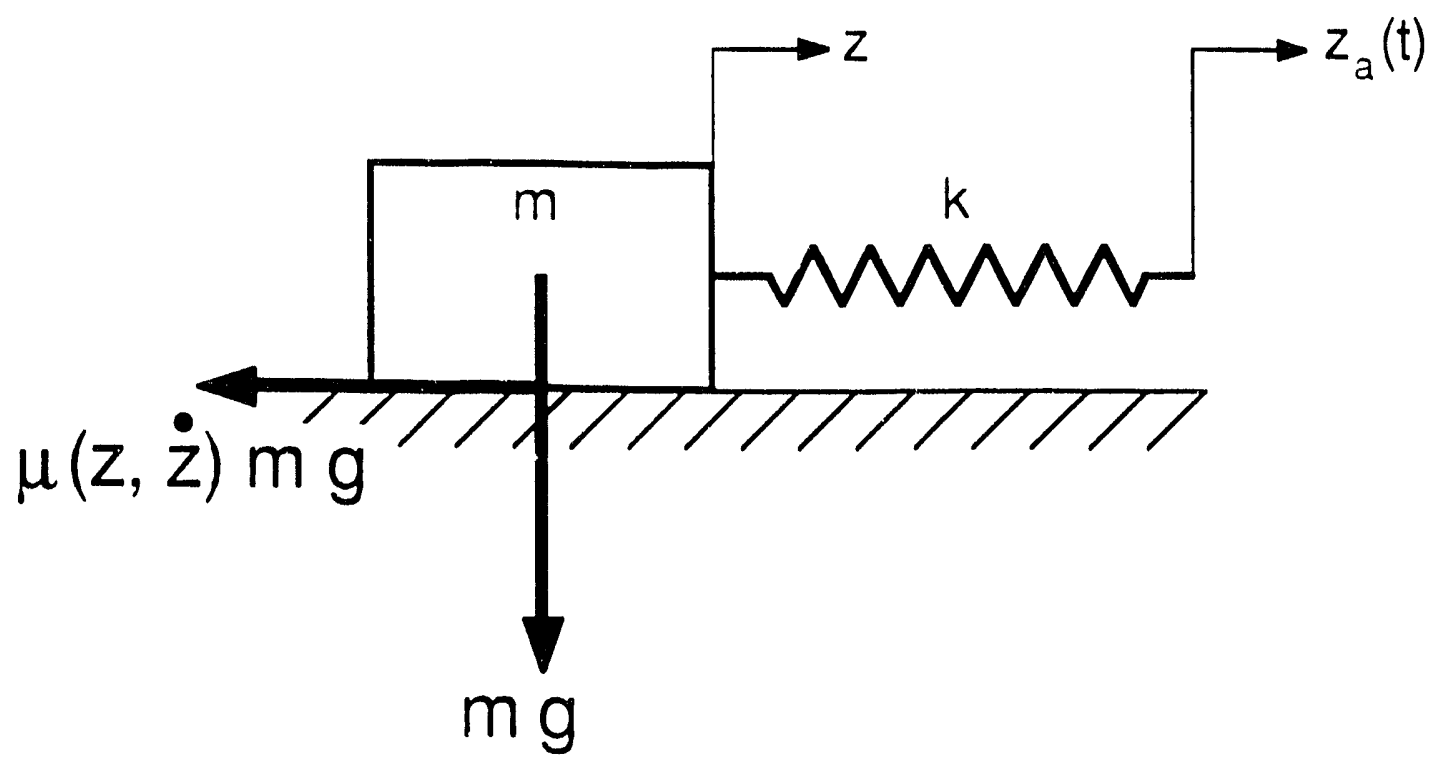

Figure 6.1: Conductor-motion model.

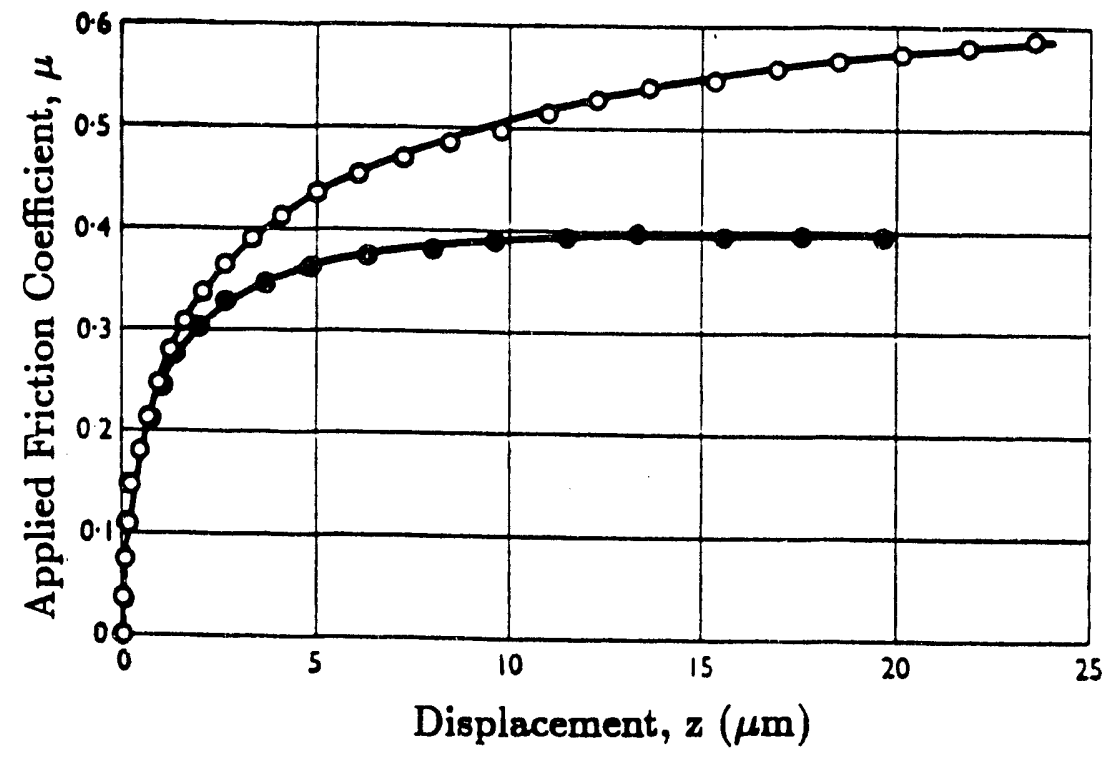

Figure 6.2: Static displacement vs applied friction coefficient. Results are for platinum-on-platimum sliding pair. Unfilled symbols are for unlubricated surfaces, filled symbols represent surfaces lubricated by solution of lauric acid in cetane. Figure from [8]. 
the interfacial junction stiffness, $k_{j}$, can be approximated:

$$
k_{j}=\frac{\mu_{\bullet} m g}{z^{*}}
$$

where $\mu_{0}$ is the limiting friction coefficient denoting the transition from near-static contact to sliding.

It should be noted that not all of Courtney-Pratt and Eisner's results can be neatly summarized by Eq. 6.1. All of the material pairs tested showed some form of time-dependent displacement following each load increment. In most instances the time-dependence decayed to vanishingly small levels after a few minutes. However, for low-melting-point metals like indium and tin these displacements persisted beyond the temporal stability of their test apparatus, even at the lowest loads. Hence, a unique "static" friction coefficient is ill defined for these metals. The non-standard frictional characteristics for these low-melting-point metals will be further discussed in $§ 6.3 .1$.

\subsubsection{Velocity-controlled stick-slip}

The system equation for the mass-spring-friction model in Fig. 6.1 is:

$$
m \ddot{z}+\mu(z, \dot{z}) m g \operatorname{sgn}(\dot{z})+k z=k z_{a}(t), \quad \text { where } \operatorname{sgn}(\dot{z})= \begin{cases}+1, & \text { if } \dot{z} \geq 0 \\ -1, & \text { otherwise. }\end{cases}
$$

A closed form solution to Eq. 6.2 is possible as long as the sign of the sliding velocity, $\operatorname{sgn}(\dot{z})$, remains constant. The imposed sliding displacement $z_{a}(t)$ is assumed zero for $t<0$ and equal to $v_{0} t$ for $t \geq 0$.

Two approximate solutions to Eq. 6.2 will be developed in the following sections. The first covers the system response prior to sliding initiation, while the second analyzes the ensuing slip portion of the stick-slip cycle. Stable sliding pairs stick only once, during sliding initiation. By contrast, unstable sliding pairs demonstrate repeated stick-slip cycles. In marginally stable pairs, no actual stoppage of motion occurs, rather the sliding behavior is characterized by a cyclical variation in the slip velocity.

The following friction coefficient will be used:

$$
\mu(z, \dot{z})=\left\{\begin{aligned}
\frac{\mu_{*}^{*} z,}{z^{*}}, & \text { if } 0 \leq z \leq z^{*} \\
\mu_{0}+\frac{\delta_{\mu}}{\delta \dot{z}}|\dot{z}|, & \text { otherwise. }
\end{aligned}\right.
$$


This friction coefficient covers the three representative friction-velocity traces from Figure 5.1, namely friction coefficients that increase with sliding speed $(\delta \mu / \delta \dot{z}>0)$, constant friction coefficients $(\delta \mu / \delta \dot{z}=0)$, and friction coefficients that decrease with increasing speed $(\delta \mu / \delta \dot{z}<0)$.

\subsubsection{Stick analysis}

The stick portion of the analysis concerns events occurring prior to sliding initiation; this analysis persists up to the time $t^{*}$ when the slider displacement reaches $z^{*}$. The frictional bond during stick is modeled in Figure 6.3 as a junction stiffness, $k_{j}=\mu, m g / z^{*}$. The corresponding system equation is given by:

$$
\ddot{z}+\left(\frac{k}{m}+\frac{k+k_{j}}{m}\right) z=\left(\frac{k}{m}\right) v_{0} t, \quad t<t^{*} .
$$

Adopting the notation $\omega_{n}^{2}=k / m$ as the natural frequency of mass-spring system, $\omega_{j}^{2}=k_{j} / m=\mu, g / z^{*}$ as the junctions' natural frequency and $\omega_{1}=\sqrt{\omega_{n}^{2}+\omega_{j}^{2}}$ as the combined system frequency, the solution to Eq. 6.4 becomes:

$$
z(t)=\frac{\omega_{n}^{2}}{\omega_{n}^{2}} v_{0} t+A \sin \left(\omega_{s} t\right)+B \cos \left(\omega_{s} t\right), \quad t<t^{*}
$$

Applying the boundary conditions that $z=0$, and $\dot{z}=0$ at $t=0$ yields

$$
z(t)=\frac{\omega_{n}^{2}}{\omega_{\theta}^{2}} v_{0} t-\frac{\omega_{n}^{2}}{\omega_{\imath}^{2}} v_{0} \frac{1}{\omega_{0}} \sin \left(\omega_{0} t\right), \quad t<t^{*} .
$$

Several characteristic responses to Eq. 6.6 are possible depending on the relation between the imposed sliding velocity $v_{0}$ and the system response time $1 / \omega_{0}$. The one that concerns us most in the present analysis is the low speed solution $\left(t^{*} \gg 1 / \omega_{0}\right)$. For this solution the slider displacement $z(t)$ increases more or less linearly with time.

The exact value of sliding system's characteristic response time is not known a priori, however, its lower-bound may be estimated using the junction frequency as a guide. Using typical values for $\mu, \sim 0.3$ and $z^{*} \sim 5 \mu \mathrm{m}$ gives a junction frequency $\omega_{j}$ of $\sim 600,000 \mathrm{rad} / \mathrm{s}$. Applying the condition that $\omega_{s} \geq \omega_{j}$ yields a lower bound estimate that $t^{*}>2 \mu \mathrm{s}$. Because the actual value of $v_{0} t^{*}$ can be no less than $z^{*}$ the low speed approximation $\left(v_{0}<z^{*} / t^{*}\right)$ is applicable for sliding speeds up to 


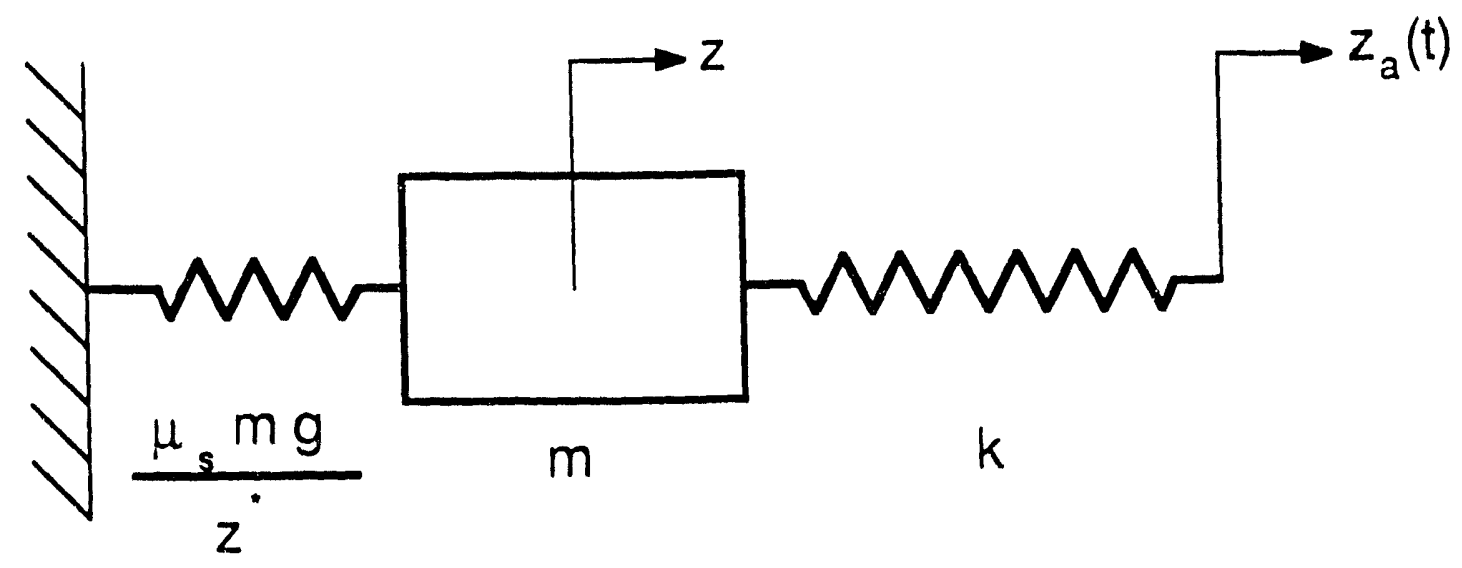

Figure 6.3: Conductor-motion model during stick portion of the stick-slip cycle.

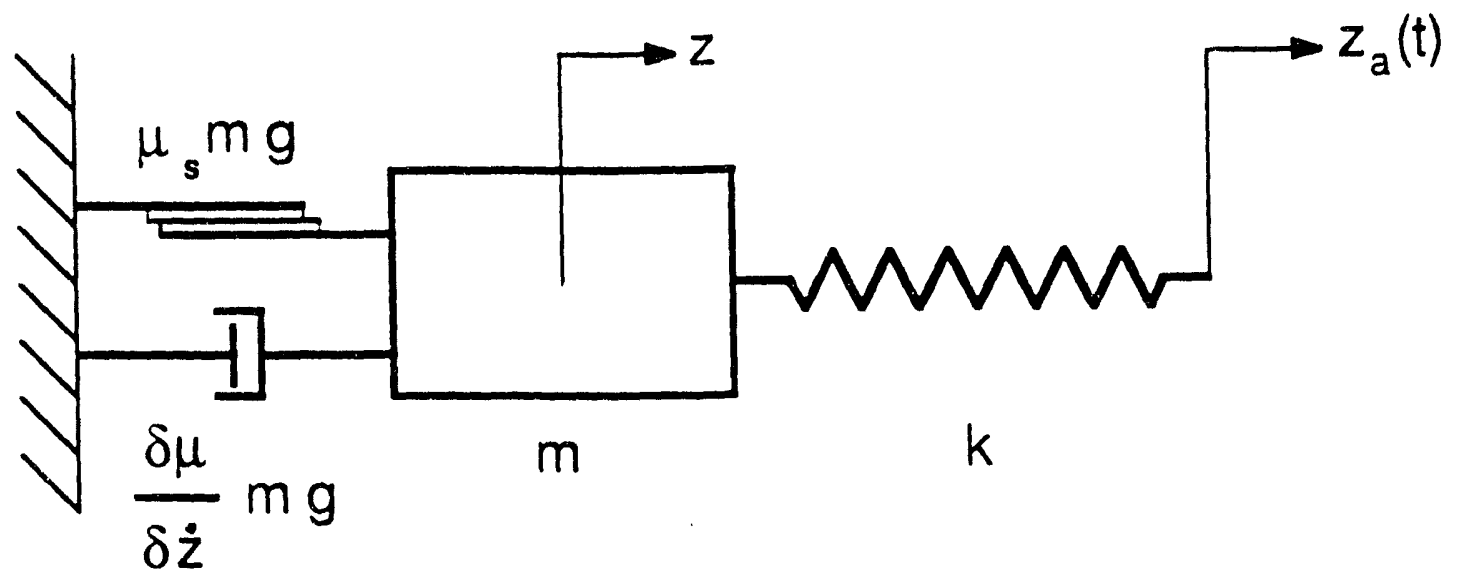

Figure 6.4: Conductor-motion model during slip portion of the stick-slip cycle. 
$\sim 3 \mathrm{~m} / \mathrm{s}$. The maximum slip velocities deduced from magnet measurements lie in range $10^{-3} \sim 10^{-2} \mathrm{~m} / \mathrm{s}$. Hence, the approximate low-speed sliding solution:

$$
\begin{aligned}
z^{*} & \approx \frac{\omega_{n}^{2}}{\omega_{s}^{2}} v_{0} t^{*}=\left(\frac{k}{k+k_{j}}\right) v_{0} t^{*} \\
\dot{z}\left(t=t^{*}\right) & \approx \frac{\omega_{n}^{2}}{\omega_{s}^{2}} v_{0}=\left(\frac{k}{k+k_{j}}\right) v_{0}
\end{aligned}
$$

provides the necessary initial conditions for the slip portion of the stability analysis.

\subsubsection{Slip analysis}

Figure 6.4 presents sliding model employed during the slip analysis. The junction stiffness, $k_{j}$, formerly used to represent the interfacial friction has been replaced by a non-linear frictional damper, representing both the static and velocitydependent portions of the friction force. The system equation during slip becomes:

$$
\ddot{z}+\left(\frac{\delta \mu}{\delta \dot{z}} g\right) \dot{z}+\left(\frac{k}{m}\right) z=\left(\frac{k}{m}\right) v_{0} t-\mu_{g} g \operatorname{sgn}(\dot{z}), \quad t \geq t^{*} .
$$

This equation will first be solved assuming a positive sliding velocity. The resulting solution will then be examined to determine the validity of this assumption. Placing Eq. 6.8 into a standard system equation format with $\zeta=(\delta \mu / \delta \dot{z})\left(g / 2 \omega_{n}\right.$ yields: ${ }^{[9]}$

$$
\ddot{z}+2 \zeta \omega_{n} \dot{z}+\omega_{n}^{2} z=\omega_{n}^{2} v_{0} t-\omega_{j}^{2} z^{*}, \quad t \geq t^{*}
$$

Applying to boundary conditions that $z=z^{*}$, and $\dot{z}=\omega_{n}^{2} / \omega^{2} v_{0}$ at $t=t^{*}=$ $\left(\omega_{s}^{2} / \omega_{n}^{2}\right)\left(z^{*} / v_{0}\right)$ produces the following the closed-form solution to Eq. 6.9

$$
\begin{aligned}
z(t)=v_{0} t & -\frac{\omega_{j}^{2}}{\omega_{n}^{2}} z^{*}-\frac{2 \zeta v_{0}}{\omega_{n}}+\frac{\left(2 \zeta^{2} \omega_{d}^{2}-\omega_{j}^{2}\right) v_{0}}{\omega_{g}^{2} \omega_{n}} e^{-\zeta \omega_{n}\left(t-t^{*}\right)} \cos \left(\omega_{d}\left(t-t^{*}\right)\right) \\
& +\frac{2 \zeta v_{0}}{\omega_{n}} e^{-\zeta \omega_{n}\left(t-t^{*}\right)} \sin \left(\omega_{d}\left(t-t^{*}\right)\right), \quad t \geq t^{*} \\
\dot{z}(t)=v_{0} & -\frac{\omega_{j}^{2}}{\omega_{d}^{2}} v_{0} e^{-\zeta \omega_{n}\left(t-t^{*}\right)} \cos \left(\omega_{d}\left(t-t^{*}\right)\right) \\
& -\frac{\zeta \omega_{n}\left(\omega_{n}^{2}+\omega_{d}^{2}\right) v_{0}}{\omega_{g}^{2} \omega_{d}} e^{-\zeta \omega_{n}\left(t-t^{*}\right)} \sin \left(\omega_{d}\left(t-t^{*}\right)\right), \quad t \geq t^{*}
\end{aligned}
$$

where $\omega_{d}=\omega_{n} \sqrt{1-\zeta^{2}}$. 


\subsubsection{Stick-slip prevention}

The intent of this analysis is not to develop a rigorous mathematical theory of stick-slip instabilities; that work has been presented elsewhere. ${ }^{[10]}$ Instead, the purpose is to provide a theoretical framework for discussing the two most common approaches towards sliding stabilization.

The easiest sliding situation to evaluate is the limiting case of a constant friction coefficient $\left(\frac{\delta \mu}{\delta \dot{z}}=\zeta=0\right)$. For a constant friction coefficient sliding pair the slip velocity (Eq. 6.10) reduces to:

$$
\begin{aligned}
& \dot{z}(t)=v_{0}-\frac{\omega_{j}^{2}}{\omega_{a}^{2}} v_{0} \cos \left(\omega_{n}\left(t-t^{*}\right)\right), \quad t \geq t^{*}, \\
& \dot{z}(t)=v_{0}-\frac{k_{j}}{k+k_{j}} v_{0} \cos \left(\omega_{n}\left(t-t^{*}\right)\right), \quad t \geq t^{*} .
\end{aligned}
$$

The slip velocity consists of a constant component equal to the imposed sliding velocity, $v_{0}$, plus an oscillatory component. The magnitude of the oscillatory part varies from near zero for a very stiff sliding system $\left(k \gg k_{j}\right)$ to a value approaching $\pm v_{0}$ for a low system stiffness $\left(k \ll k_{j}\right)$. Thus, the most reliable method for reducing the oscillatory part of the slip velocity is to increase the system stiffness to a point where the potential slip distance remains less than the average junction diameter; this method has been found to work even for sliding systems with slightly negative friction-velocity characteristics. ${ }^{[1,2]}$

The conductors employed in contemporary high-performance magnets are presently optimized with regard to their electromagnetic characteristics rather than their high intrinsic stiffness. In fact, extremely stiff conductors are generally undesirable from a manufacturing viewpoint in that they make the magnet much more difficult to wind. Hence, instead of reoptimizing conductors for improved frictional stability the conventional approach has been to approach the problem from the standpoint of optimizing the frictional properties of contemporary construction materials.

The dynamic system equation (Eq. 6.9) confirms that this materials-based approach to frictional stabilization is a valid method for eliminating unwanted stick-slips. Equation 6.9 represents the system equation for a damped harmonic 
oscillator. ${ }^{[9]}$ When the damping coefficient is negative $\left(\zeta \propto \frac{\delta \mu}{\delta \dot{i}}<0\right)$ the exponential part of the solution grows without bounds; however, because of the sign of the friction force changes as $\dot{z} \rightarrow 0$ the sliding speed does not actually blow-up, rather the sliding behavior passes into a series of unstable stick-slips. Conversely, for positive damping coefficients $\left(\zeta \propto \frac{\delta \mu}{\delta \dot{\boldsymbol{z}}}>0\right)$ the oscillatory sliding component eventually decays away. In fact, for sufficiently large damping coefficients the slider's velocity tracks the imposed velocity without any overshoot. If abrupt conductor motions are to be prevented in the presence of a relatively low conductor stiffness $\left(k \ll k_{j}\right)$ material pairs with appreciably positive friction-velocity characteristics $\left(\frac{\delta \mu}{\delta \dot{z}} \gg \mathrm{C}\right)$ must be found.

The use of positive friction-velocity characteristics has been recognized by the superconducting magnet community for a number of years. ${ }^{[11,12]}$ For the most part the search for stable cryogenic friction materials has been conducted as an empirical investigation of contemporary construction materials.

Several material selection guidelines have been developed within the framework of the adhesive friction theory to guide the development of stable room-temperature sliding materials. ${ }^{[1,13-18]}$ In Ch.4 the adhesion theory of friction and wear was shown to remain valid at cryogenic temperatures, once temperature-dependent variations in material strength properties are taken into account. $\S 6.2$ and 6.3 re-examine the two most common classes of stable room-temperature sliding materials to assess how well materials-based stabilization techniques extrapolate to cryogenic temperatures. The material classes include viscoelastic polymers and low-melting-point crystalline solids at temperatures above about half their melting points.

\subsection{Viscoelastic sliding stabilization}

A large portion of early stick-slip research was sponsored by the rubber tire industry to aid in the development of skid resistant rubber tires. The large, recoverable elastic strain limit possessed by most elastomers makes them especially suitable candidates for developing correlations between their bulk physical properties and their frictional characteristics.

The strain increment experienced by each asperity contact during sliding typically ranges to a few tenths. ${ }^{[4-6]}$ For most materials these strain increments pro- 
duce a combined elastic-plastic deformation of the asperity junctions. Rubbers are unique in that they generally demonstrate fully recoverable deformations in the range 100 500\%. Because little permanent change occurs in the elastomer's morphology during sliding its bulk viscoelastic damping characteristics are well correlated with its frictional behavior.

\subsubsection{Regions of mechanical equivalence.}

Before discussing the correlation between elastomeric mechanical properties and frictional behavior it may be helpful to first review to the general mechanical characteristics of viscoelastic polymers. ${ }^{[16]}$

Polymeric materials are generally viscoelastic, that is, their mechanical properties depend both on their use temperature and their time under load. Stress relaxation experiments provide a simple means for characterizing a polymers viscoelastic behavior. To conduct a stress relaxation experiment, a geometrically uniform specimen is clamped at one end and suddenly subjected to a uniform tensile strain, $\epsilon_{0}$. The resulting stress, $\sigma(t)$, is then measured as a function of time. For small values of strain the material's stress relaxation modulus, $E_{r}(t)$, is determined by dividing the stress at time $t$ by the applied strain, $E_{r}(t)=\sigma(t) / \epsilon_{0}$.

There are two common methods for presenting stress-relaxation data. The first is to plot the relaxation modulus at a fixed time, $t=t_{0}$, vs various test temperatures; this data is known as the isochronal relaxation modulus. A frequently used relaxation time interval is $10 \mathrm{~s}$. The second method is to plot the relaxation modulus at a fixed temperature $v$ s the logarithm of the time under load; this data is known as the isothermal relaxation modulus. Because the stiffness of a polymer may extend over several orders of magnitude, the relaxation modulus is scaled logarithmically to present the range of data values in a conveniently short space.

Figure 6.5 presents a schematic plot of the relaxation modulus for a typical amorphous polymer. The plot is divided into four regions, representing the four most common classifications of polymeric behavior. The time- and temperaturedependent properties of all amorphous polymers correspond more or less with the regions depicted in Figure 6.5.[16] However, the transition between regions varies uniquely with the polymer's morphology and thermal characteristics. 


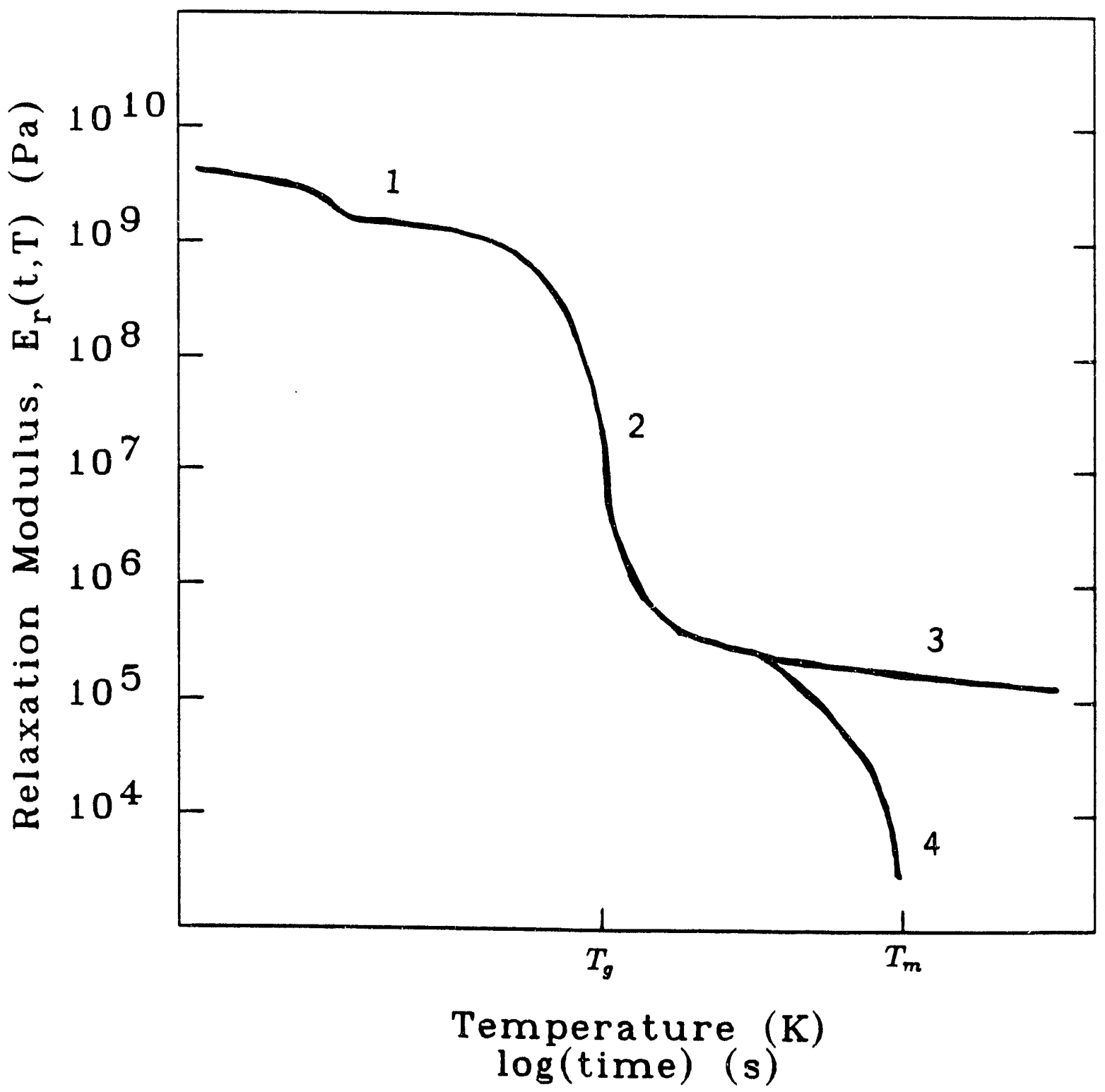

Figure 6.5: Schematic representation of the relaxation modulus, $E_{r}(t, T)$, for a typical viscoelastic polymer $v s$ a temperature or $\log ($ time $)$ axis. 
The low-temperature/short-loading-time portion of the graph (Region 1, upper left) depicts the behavior of polymer glasses. The modulus in this region takes on values in the range $1-10 \mathrm{GPa}$. A glassy polymer is stiff, hard, and generally brittle; its behavior is well described by the theory of linear elasticity. Glassy polymers demonstrate virtually time-independent behavior. On the molecular level, elastic deformation in the glassy region is achieved by numerous, short-chain-segment rotations. ${ }^{[17]}$ Each chain segment rotation typically encompasses a few adjacent repeat units.

In transition region (Region 2) the amorphous polymer's modulus drops from 1 GPA to $1 \mathrm{MPa}$. A transition-region polymer demonstrates highly time- and temperature-dependent properties; its small-strain behavior is well represented by the theory of linear viscoelasticity. ${ }^{[16]}$ The transition region defines one of the most important thermal characteristics of a polymer, namely its glass transition temperature, $T_{g} . T_{g}$ is often approximated by the temperature at which the 10-s isochronal relaxation modulus reaches a value of $3 \times 10^{7} \mathrm{~Pa}$. The glass transition temperature generally lies between one half and two thirds of the polymers melting temperature.

Viscoelastic effects are also observed, to a much more limited extent, in semicrystalline polymers. The crystalline regions stiffen the polymer by restricting longrange chain segment motions. The relaxation modulus in a semicrystalline polymer seldom drops below $\sim 3 \times 10^{7} \mathrm{~Pa}$.

The rubber-like behavior that characterizes Region 3 is observed only in amorphous polymers. Two important cases can be distinguished depending on whether the rubber is cross-linked or not. Cross-linked rubbers are capable of recoverable deformations on the order of $500 \sim 1000 \%$ whereas uncross-linked rubbers recover fully only if stretched to a small extent for short periods of time. The modulii in the elastomeric region lie typically between 0.1 and $1 \mathrm{MPa}$. Mechanical behavior in the elastomeric region is time-independent and characterized the the theory of rubber elasticity. ${ }^{[16,18]}$ The chain segment motions during elastomeric deformation typically involves hundreds of repeat units, or roughly the entire chain length between cross-links. The transition region (Region 2) thus represents a change from molecular motions encompassing single repeat units to motions involving entire chain lengths. 
The fourth region of polymer behavior is observed in uncross-linked rubbers. At high temperatures, or long loading times, uncrosslinked polymers eventually lose their ability to recover from deformation. At sufficiently high temperatures/long loading times Region 4 polymers behave as liquids with high viscosity and low, but non-zero, elasticity. The temperature at which viscous flow begins to dominate the polymer's behavior is denoted by the polymer's melting temperature, $T_{m}$.

The most compelling facet of polymer behavior is that once the relaxation modulus of an amorphous polymer has been measured for a single set of test conditions, its behavior for any combination of loading-time and temperature is uniquely specified and can be determined by transforming the original data set to the desired operating conditions using the Williams-Landel-Ferry (WLF) transform. ${ }^{[19]}$ The original set of relaxation data forms what is known as the polymer's mechanical relaxation master curve. The transition from one set of test conditions to another is accomplished using a shift factor, $a_{T}$, which horizontally displaces the master curve relative to itself in proportion either to difference between $T$, the desired use temperature, and $T_{g}$, or in proportion to the ratio between the desired loading time, $t$, and the isochronal measurement time, $t_{0}$

$$
\log \left(a_{T}\right)=\log \left(\frac{t}{t_{0}}\right)=\frac{-17.44\left(T-T_{g}\right)}{51.6+\left(T-T_{g}\right)}
$$

Eq. 6.12 indicates that a. 3-K temperature increase near $T_{g}$ produces approximately an order of magnitude decrease in the polymer's mechanical relaxation time.

\subsubsection{Time-temperature superposition in elastomer friction}

The most comprehensive experimental investigation of elastomeric friction was the pioneering work performed by Grosch. ${ }^{[14]}$ Figure 6.6 presents Grosch's original friction-velocity-temperature data for an acrylonitrile-butadiene rubber slid against textured glass. Figure 6.6a shows individual friction coefficient vs velocity traces obtained at several fixed test temperatures. By application of the WLF equation Grosch was able to combine these individual friction traces into a single frictionvelocity master curves, covering a much broader range of velocities than was experimentally accessible. Grosch's acrylonitrile-butadiene friction master curve is reproduced in Figure 6.ub. The reference temperature for Figure $6.6 \mathrm{~b}$ is $293 \mathrm{~K}$. By contrast, the acrylonitrile-butadiene's glass transition temperature is $\sim 250 \mathrm{~K}$. 
Similar frictional correlations have been produced for other elastomers. ${ }^{[14,15]}$

An important feature to note about the friction master curve is that the friction coefficient does not continue to increase indefinitely. Rather, as the sliding speed increases the friction coefficient rises rapidly at low speeds, passes through a peak value, and then decreases again almost as rapidly. If $v_{p e a k}(T)$ is used to denote the sliding speed corresponding to the peak friction coefficient at temperature $T$, then a necessary condition for ensuring stable sliding is that the imposed sliding velocity, $v_{0}$ (from $\S 6.1 .3$ ), must remain less than $v_{\text {peak }}(T)$.

The sliding velocities measured during conductor motion experiments typically range up to $\sim 10 \mathrm{~mm} / \mathrm{s}$; this sets an approximate limit for the desired $v_{\text {peak }}(T)$. For instance, if the slip velocity during an $\sim 10 \mu \mathrm{m}$ conductor displacements could be reduced from $\sim 10$ to $\sim 0.1 \mathrm{~mm} / \mathrm{s}$. Figure 3.2 indicates that conductor-motioninduced quenches could be markedly reduced.

\subsubsection{Viscoelastic friction model}

Several models have been proposed to account for the close similarity between elastomeric friction-velocity-temperature effects and a rubber's bulk mechanical characteristics. ${ }^{[15,20-22]}$ Ludema and Tabor propose a somewhat intuitive explanation for the origins of elastomeric friction-velocity peaks based on the adhesive friction theory's description of the friction force as a combination of area- and shearstrength-dependent effects. ${ }^{[15]}$

$$
F(v)=A(v) s(v)
$$

They contend that velocity-dependent contact area $A(v)$ is determined by the conditions of the friction experiment, namely, that each interfacial junccion persists for a time $t^{*}$ determined by the ratio of sliding speed to the average junction size, $t^{*}=v_{0} / z^{*}$. Because of an elastomer's large elastic limit, its contact area is determined not by its penetration hardness, but rather by its time-dependent relaxation modulus, $E_{r}(t)$. For a single Hertzian contact the velocity-dependent elastic contact area becomes:

$$
A(v)=\frac{k}{E_{r}\left(\frac{v}{z^{v}}\right)^{\frac{2}{3}}}
$$

$k$ is an unspecified scaling parameter used to equalize the predicted and measured friction values. 

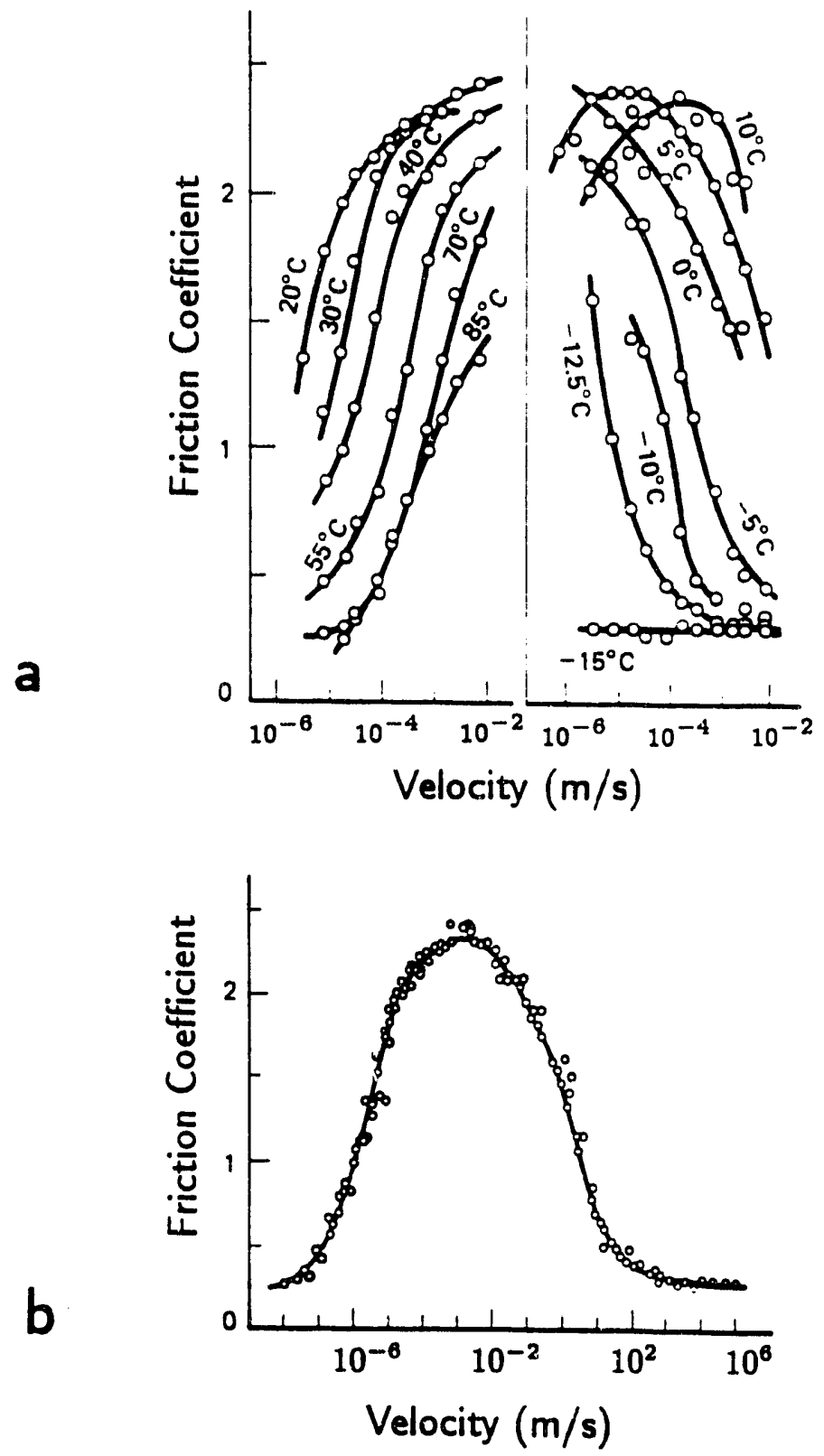

Figure 6.6: Viscoelastic, friction-velocity-temperature data for an acrylonitrilebutadiene rubber. a) Individual friction $v s$ velocity traces at fixed temperatures. The data is separated into two graphs for clarity. b) Acrylonitrile-butadiene friction-velocity-temperature master curve, obtained using WLF shift factor and a $293 \mathrm{~K}$ reference temperature. Data from [14]. 
Ludema and Tabor likewise contend that the interface between a rubber and another unlubricated sliding surface is nearly as strong as the rubber itself. Although strain-rate- and temperature-dependent shear stress data were not available at the time of their experiments, they did have access to tensile rupture data, $\sigma_{r}(\dot{\epsilon})$ which indicated that a rubber's failure stress also conforms to a single master curve via the WLF transformation. They adopted this tensile rupture data as an approximate measure of the junction shear stress, $s$.

Figure 6.7 presents Ludema and Tabor's attempt to correlate the deformationrate-dependent bulk material properties of an acrylonitrile-butadiene rubber with its friction-velocity curve. To develop this correlation the strain-rate dependent rupture data, $\sigma_{r}(\dot{\epsilon})$, was converted to the requisite velocity-dependent shear stress, $s(v)$, by assuming that the interfacial shear strain is confined to a thin $(\sim 100 \AA$ thick $)$ surface layer, corresponding roughly to the average distance between polymer cross-links. A representative junction of size of $z^{*}=1 \mathrm{~mm}$ was similarly selected to set the relative position of the low-speed portion of the $A(v)$ curve. Evidence in support of such large elastomeric junction sizes has recently been provided based on a comparison of static and kinetic friction measurements. [23]

The composite friction velocity curve in Figure 6.7 is in good qualitative agreement with the experimental data presented in Figure 6.6b. However, Ludema and Tabor's explanation is not without its difficulties. For instance, if the actual value of the Hertzian contact area is used, the predicted friction force is about an order of magnitude larger than is measured experimentally. Despite this shortcoming, the analysis does serve to highlight the importance of the individual terms $A(v)$ and $s(v)$ in determining frictional behavior.

As the sliding speed increases the contact area decreases, while the shear strength increases. Low-speed friction combines a large contact area with a low shear stress resulting in low overall friction. Conversely, high-speed friction combines a small contact area with a high shear stress, hence friction is also low. The occurrence of a peak friction value during the cross-over between low-speed and high-speed sliding is attributed to the fact that the deformation processes responsible for $s(v)$ occur on a physical scale approximately five orders of magnitude smaller than the deformation processes responsible for $A(v)$. ${ }^{[15]}$ 


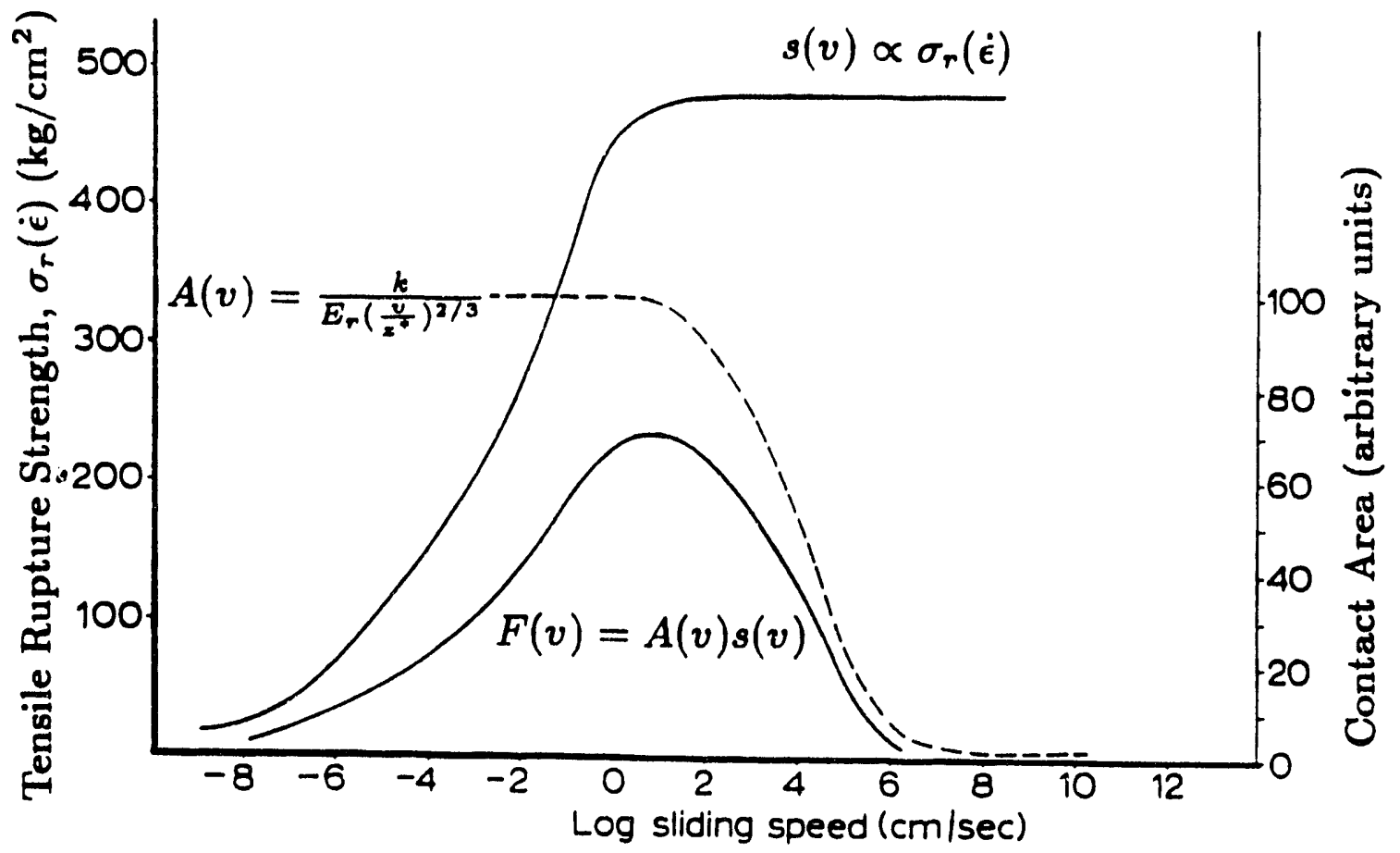

Figure 6.7: Schematic representation of viscoelastic friction model. The model proposes that the viscoelastic friction peak occurs because of competition between velocity-dependent changes in both interfacial shear strength and interfacial contact area. Figure from [15]. 


\subsubsection{Friction-temperature behavior}

Thus far, the viscoelastic friction data in the chapter have been presented in the standard format, as friction vs velocity traces. From a practical standpoint, however, the chapter is more concerned with extrapolating these viscoelastic friction correlations to very low temperatures. Figure 6.8 replots Grosch's acrylonitrilebutadiene friction as friction $v s$ temperature traces at several fixed sliding speeds.

Figure 6.8 illustrates several important characteristics of viscoelastic sliding stability. The first is that velocity-dependent friction effects are not prominently observed until the test temperature exceeds the polymers glass transition. $T_{g}$ for this acrylonitrile-butadiene rubber is $\sim 250 \mathrm{~K}$.

A second feature brought out by friction-temperature traces it that the slope of the friction-velocity curve assumes negative values $(\delta \mu / \delta \dot{z}<0)$ in the temperature region immediately above $T_{g}$. This observation is consistent with Luderna and Tabor's viscoelastic friction model. The low-temperature portion of each friction temperature trace represents the combination of a low contact area and high interfacial shear stress. As the temperature increases above $T_{g}$ the contact area increases rapidly, the shear stress remains relatively constant, and the friction increases. Similarly, as the sliding speed increases the area-dependent friction increase is offset to higher temperatures because of the corresponding reduction in the asperity contact time. This velocity-dependent shift in the low-temperature portion of the friction-temperature traces thus gives rise to the rubber's unstable frictionvelocity characteristic.

\subsubsection{Low-temperature polymer friction}

Figure 6.9 shows friction vs velocity traces for a soft (Durometer 30) silicone rubber sliding against oxygen-free-high-conductivity OFHC copper balls. The tests were performed at 293 and $77 \mathrm{~K}$ using the pin-on-disk tribometer discussed in \$4.2.3.1. The normal load was $7.5 \mathrm{~N}$. With $T_{g}=150 \mathrm{~K}$, silicone rubber has the lowest known glass transition temperature of any polymer. Hence, it is the most likely elastomer to show some vestige of velocity-dependent frictional behavior at cryogenic temperatures.

The 293-K peak-friction velocity for this silicone rubber lies in excess of 


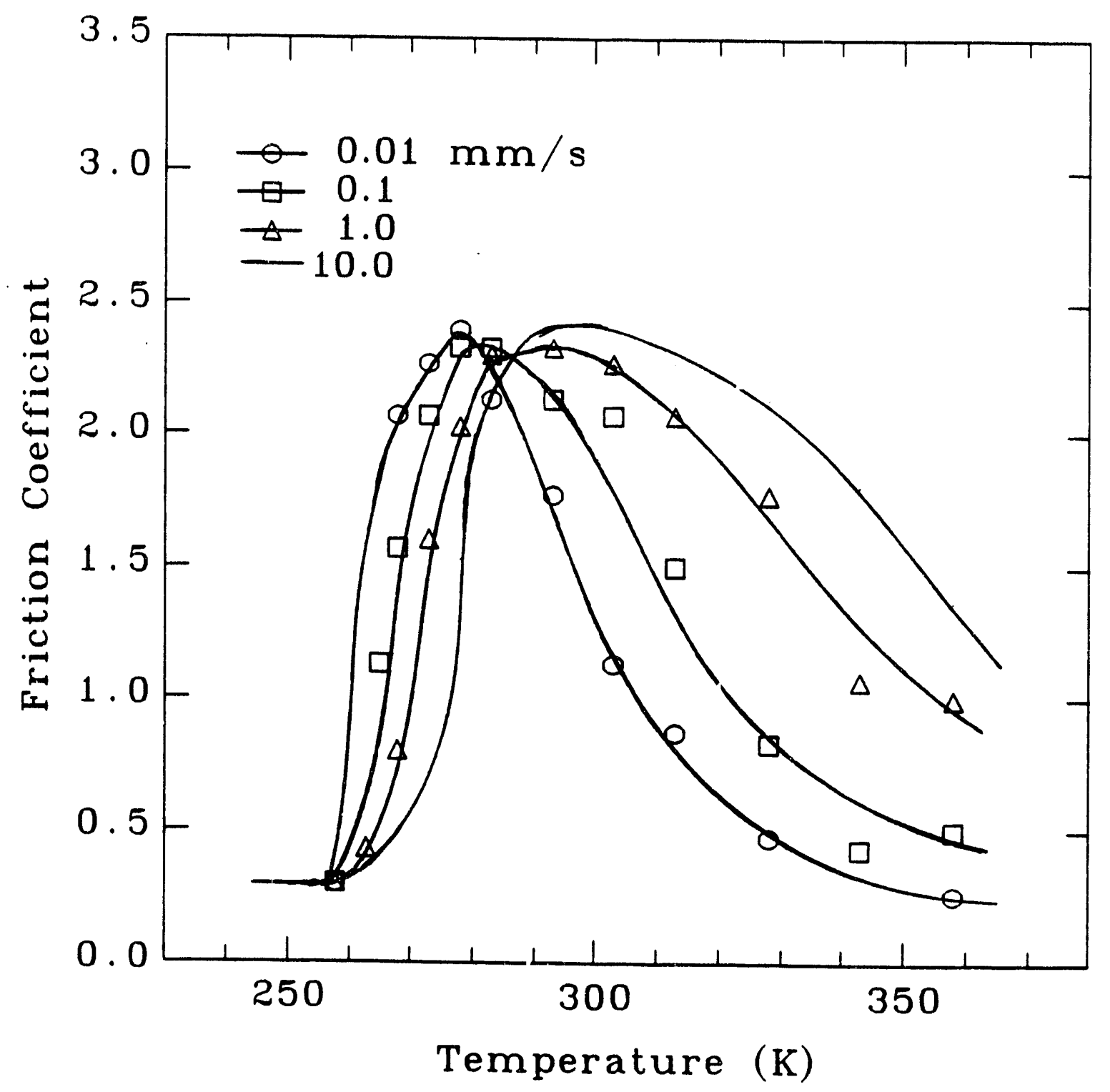

Figure 6.8: Grosch's acrylonitrile-butadiene data replotted as friction vs temperature traces. Data for four speeds are shown, $1 \times 10^{-5}, 1 \times 10^{-4}, 1 \times 10^{-3}$, and $1 \times 10^{-2} \mathrm{~m} / \mathrm{s}$. 


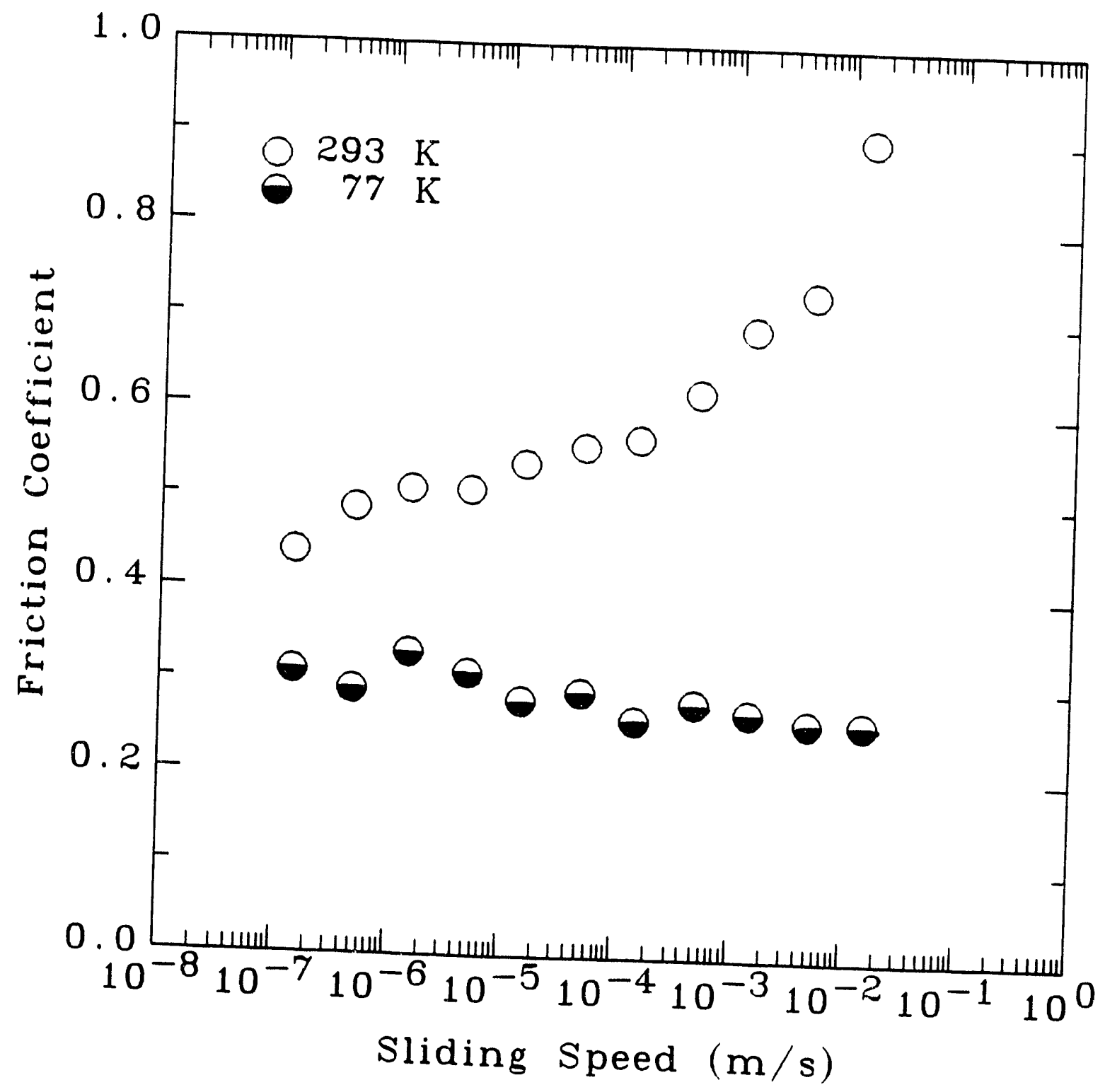

Figure 6.9: Friction vs velocity trace for soft (Durometer 30) silicone rubber against AISI 316 stainless steel at 77 and $293 \mathrm{~K}$. 
$10^{-2} \mathrm{~m} / \mathrm{s}$. When the sliding temperature is lowered from room temperature $(293 \mathrm{~K})$ to $T_{g}(150 \mathrm{~K})$ the WLF equation predicts that $v_{\text {peak }}(T)$ will shift to the left by approximately 13 orders of magnitude. As the temperature decreases still further, from $T_{g}$ to $77 \mathrm{~K}$, the velocity peak continues to shift to the left so that any hope of observing the characteristic viscoelastic frictional peak passes on to a geological time scale. By $77 \mathrm{~K}$ the frictional properties of the silicone rubber are essentially that of a hard polymer glass; friction is low and virtually independent of the sliding speed. If anything, the silicone rubber acquires a slightly negative $77-\mathrm{K}$ friction-velocity characteristic.

In addition to the main-chain viscoelastic relaxation at $T_{g}$ many polymers also demonstrate a variety of sub- $T_{g}$ relaxations involving the thermally activated vibration of relatively short (three to four repeat unit) chain segment lengths. The size of the chain segments participating in the relaxation generally decreases with decreasing temperature. ${ }^{[24]}$ Sub- $T_{g}$ relaxations generally have a much more subtle, but equally observable effect, on the polymer's bulk mechanical properties as the main chain relaxations. Some of the mechanical properties influenced by sub- $T_{g}$ relaxations include: heat capacity, thermal expansion, elastic modulus, viscoelastic damping, tensile strength, toughness, and strain to failure. ${ }^{[24]}$

A good correlation between these sub- $T_{g}$ mechanical relaxations and polymeric friction has only been observed in the case of rolling friction, where the subsurface deformations are predominantly elastic. ${ }^{[15,25]}$ Although temperature-dependent changes in low-temperature polymeric friction are sometimes observed these cannot normally be attributed, other than as a second-order effect, to a corresponding lowtemperature relaxation. ${ }^{[15]}$ Low-temperature polymeric friction is most commonly attributed to plastic shearing rather than to viscoelastic damping because the shear strains produced during sliding extend into the polymer to a depth several times larger than the chain segment lengths participating in these relaxations. ${ }^{[25]}$ Thus, the key feature distinguishing between high- and low-temperature friction is that above $T_{g}$ a polymer's frictional behavior is determined by its viscoelastic material response whereas far below $T_{g}$ its behavior is dictated by the ratio of its plastic strength parameters. 


\subsubsection{Screening technique for low-temperature friction materials}

The separation of the friction force into separate area and shear-strength contributions suggests the use of time-dependent hardness measurements as a simple means for assessing a polymer's low-temperature frictional stability; this is especially true because deformation-rate-dependent changes in the contact area tend to occur at much lower temperatures than the corresponding changes in shear stress.

Figure 6.10 shows time-dependent hardness measurements for a few cross-linked epoxy resins as well as their velocity-dependent friction coefficients. The epoxy resins used during this study were compounded using Shell Chemical Company's Epon 815 resin combined with Shell's V-40 curing agent. This epoxy resin combination is frequently used as a filler in high-performance superconducting magnets constructed in our laboratory. The concentration range of curing agent recommended by the manufactured ranges from 33- to 133-phr, where the phr designation refers to the weight of curing agent in parts to one hundred parts resin. In general, the cured mixes become increasingly rubbery as their phr values increases. Representative results for a 33- and a 133-phr resin are included in Figure 6.10.

Time-dependent hardness measurements were performed for each mix at 293and 77-K using an indentation test apparatus similar to the one described in \$4.2.2. The hardness values (Figure 6.10a) were collected at fixed indentation times between 5 and $1000 \mathrm{~s}$. The indentor load during the room temperature tests was $2 \mathrm{~N}$ for the 33-phr mix and $0.15 \mathrm{~N}$ for the 133-phr resin. For the $77-\mathrm{K}$ tests a $2 \mathrm{~N}$ load was used for both mixes. Figure $6.10 \mathrm{a}$ indicates that the measured hardness of the epoxy mixes is a linearly decreasing function of time when plotted on a log-log scale. Each data point in Figure 6.10a represents the average value of at least three readings. The variation in these readings is typically within $5 \%$ at room temperature and within $10 \%$ at $77 \mathrm{~K}$. The $77-\mathrm{K}$ hardness values are relatively constant and noticeably higher than the room-temperature measurements. Of the test conditions investigated only the 133-phr mix at room-temperature shows a marked variation in its time-dependent hardness.

Figure $6.10 \mathrm{~b}$ presents the 293 - and $77-\mathrm{K}$ friction $v s$ velocity results for the epoxy mixes. The friction coefficient for each sliding combination was repeatable 

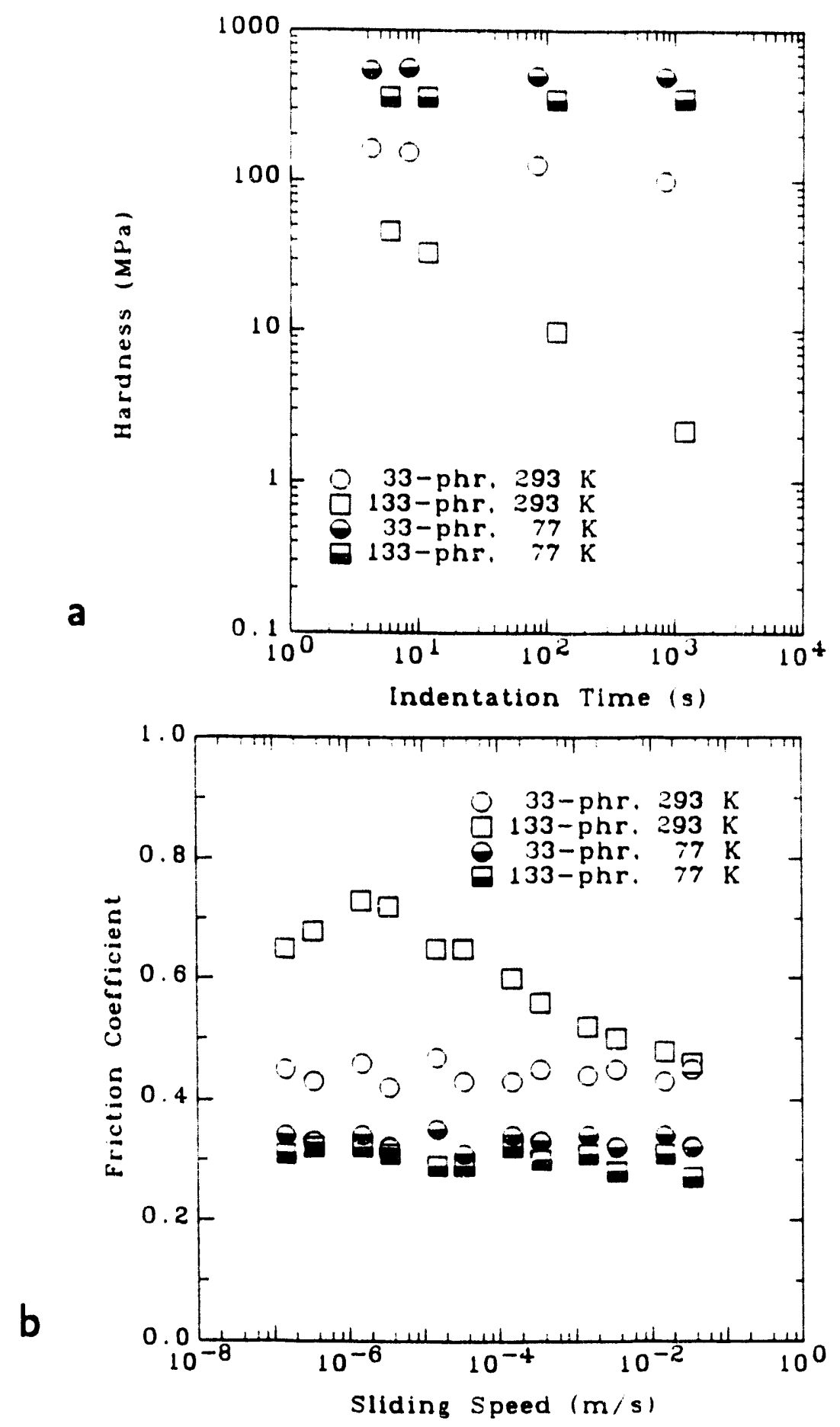

Figure 6.10: Time-dependent hardness values and velocity-dependent friction coefficients for two compositions of Epon-815 epoxy. a) Time-dependent hardness measurements at 77 and $293 \mathrm{~K}$. b) Velocity-dependent friction coefficients at 77 and $293 \mathrm{~K}$. 
to within $10 \%$. The kinetic friction coefficients for the epoxy mixes at $77 \mathrm{~K}$ are virtually identical, independent of sliding speed, and much lower than their roomtemperature values. The temperature-dependence of the friction coefficient is consistent with the mixes' measured hardness values; the friction coefficients decrease as the hardness increases (contact area decreases). By contrast, the 133-phr mix exhibits marked variations in both its velocity-dependent friction coefficients and its room-temperature hardness values. These room-temperature results are consistent with Ludema and Tabor's friction model. The pronounced variation in the 133-phr resin's room-temperature hardness (contact area) is accompanied by velocity-dependent frictional effects.

\subsubsection{Low-temperature sliding initiation}

The occurrence of a time-varying hardness value does not necessarily guarantee the presence of a peak value in friction-velocity curve. This is especially true of sliding initiation where the static contact (stick) time prior to slip can be arbitrarily long in comparison to the time, $t^{*}$, required to traverse the initial junction diameter.

Figure 6.11 plots the static friction coefficient vs stick time for the 133-phr Epon epoxy. The static friction coefficients were measured using the linear friction apparatus discussed in $\$ 5.1 .4 .1$. Figure 6.11 presents the average friction coefficient values from two test runs. During the first run the stick time, prior to the normal force application, was increased in logarithmic steps between 2 and 10,000s. During the second run, the test sequence was reversed; the stick time decreased between measurements. The friction results were reproducible to within $10 \%$ between runs. The normal force was $100 \mathrm{~N}$ and the tangential force ramping rate, $50 \mathrm{~N} / \mathrm{s}$. The use of a constant loading rate ensures that the strain rate, and hence the junction shear stress, remains essentially constant during sliding initiation.

The static friction results for the 133-phr resin are consistent with its timedependent hardness measurements (Figure 6.10a). The hardness of the 133-phr mix remains essentially fixed at $77 \mathrm{~K}$ hence its static friction coefficient also remains constant. The $77-\mathrm{K}$ static friction value is $\sim 0.23$. The time-dependent decrease in the resin's room-temperature hardness (increase in interfacial area) raises its static friction coefficient from a value of 0.54 , observed at a 2 -s stick time, to 0.77 after 


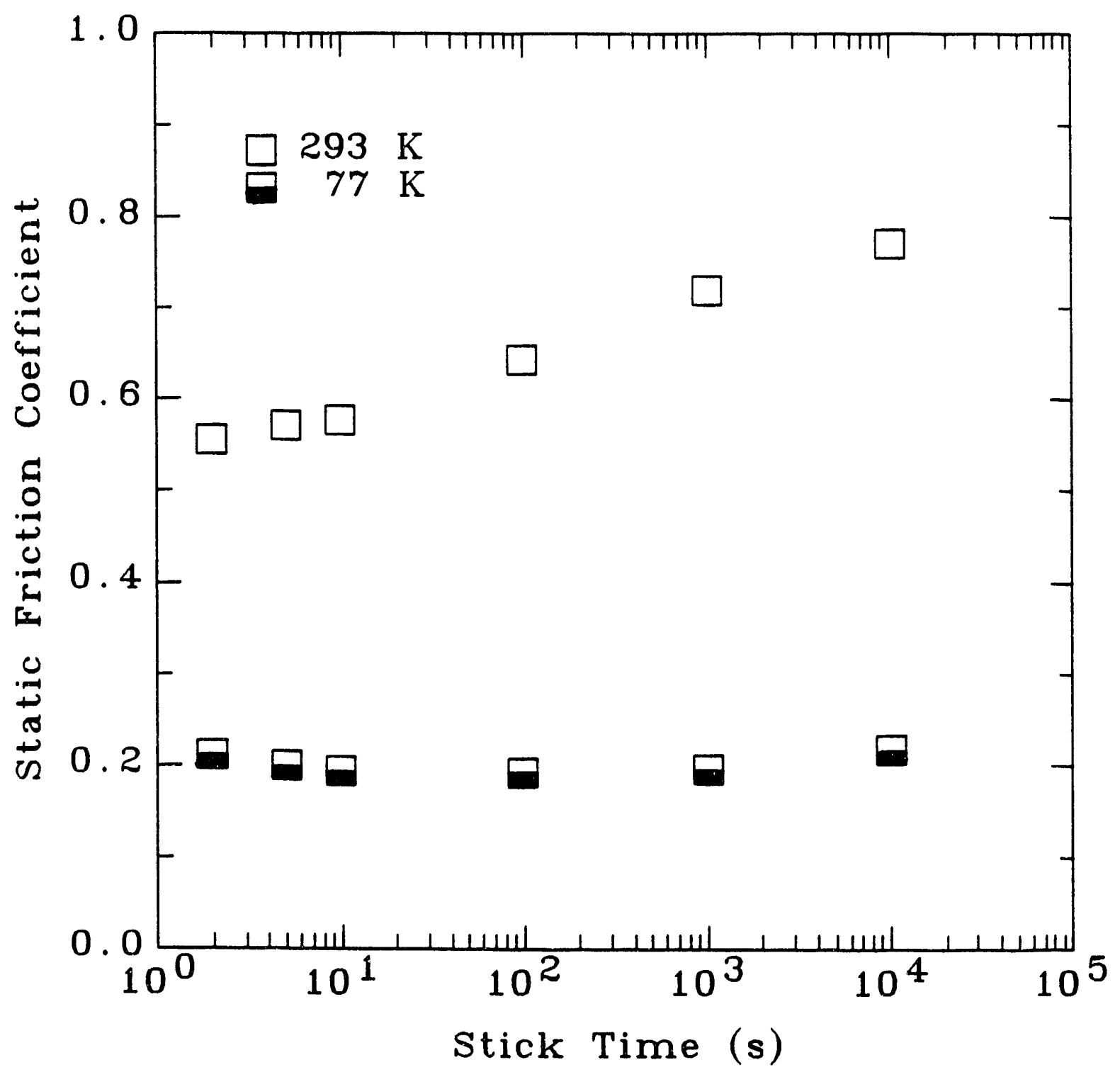

Figure 6.11: Static friction coefficient vs stick time for a 133-phr Epon epoxy at 77 and $293 \mathrm{~K}$. 
a static contact time of $10,000 \mathrm{~s}$. As sliding begins the time available for junctions growth decreases, the friction coefficient drops, and unstable slip ensues.

The negative effect of interfacial creep on $4.2-\mathrm{K}$ transient sliding stability was previously noted with regard to semicrystalline polymers in §5.1.4.2. Maeda likewise suggests that transient creep may be responsible for the training effects observed in dry-wound solenoids. ${ }^{[26]}$ The cross-linked Formvar insulation typically used in high-performance magnets is moderately viscoelastic at room temperature; this provides it with good toughness and high abrasion resistance. However, the Formvar's viscoelastic behavior also allows for a period of marked junction growth between magnet construction and cool-down to the magnet's $4.2-\mathrm{K}$ operating temperature. As the magnet cools the junction shear strength increases markedly. Because there is little impetus for the contact area to change during cool-down the friction is expected to increasing accordingly.

Maeda experimentally examined the consequences of this room-temperature junction growth using a linear-type friction apparatus and a pair of Formvar coated copper blocks. ${ }^{[2]}$ The blocks were first clamped together at room-temperature before cooling to $4.2 \mathrm{~K}$ where the transient friction experiments were performed. Two types of friction behavior were observed. The static friction coefficient during the first run was double the value observed during subsequent slips, while the motion was unstable and quick. The normal load was then released and reapplied at $4.2 \mathrm{~K}$; the resulting motion turned out to be relatively smooth and stable. Thus, although time-dependent polymer deformation is unlikely to improve cryogenic frictional stability there is ample evidence to suggest that it occurrence can seriously degrade low-temperature sliding initiation.

A frequently used technique for reducing the tendency towards this timedependent junction growth is to apply a low-amplitude, high-frequency mechanical signal to the frictional interface. An applications of this technique is found, for instance, in the practice of tapping on a rusty bolt with a hammer prior to removal. The vibration upsets the frictional bonds and reduces the static friction to values corresponding to relatively short static-contact times. No attempt has yet been made to adapt this vibrational approach for stabilizing the frictional behavior of high-performance: superconducting magnets. 


\subsection{Crystalline solids}

Crystalline materials, especially low-melting-point pure metals, also demonstrate time- and temperature-dependent frictional effects similar to those reported for the viscoelastic polymers. Figure 6.12 shows friction-velocity traces for an indium disk slid against three AISI 316 stainless steel at 293,77 , and $4.2 \mathrm{~K}$. The tests were performed using the pin-on-disk apparatus described in $\S 4.2 .3 .1$. The normal force was $7.5 \mathrm{~N}$. Friction for the indium disk at $293 \mathrm{~K}$ is high and demonstrates a pronounced friction-velocity peak. As the temperature is decreased to $77 \mathrm{~K}$ the peak in the friction-velocity curve presumedly shifts to the left so that only the descending portion of the curve is experimentally observed. The friction coefficient at $4.2 \mathrm{~K}$ is low and virtually independent of sliding speed. The same general trends in crystalline friction-velocity-temperature characteristics have previously been noted in the tribological literature. ${ }^{[13,27-29]}$ The transitions between the three sliding behaviors will is qualitatively discussed in $§ 6.3 .3$.

\subsubsection{Creep theory of friction}

\subsubsection{Positive friction-velocity characteristics}

The relation between room-temperature interfacial creep and the positive slope of the friction-velocity curve was previously investigated for indium and lead specimens by Burwell and Rabinowicz. ${ }^{[13]}$ Similar to the the tin- and indium-results reported by Courtney-Pratt and Eisner (\$6.1.2.), they observed continuously increasing displacements between their sliding specimens for any value of applied friction coefficient. The displacements, following the friction force application, were rapid at first but settled over a period weeks to near constant displacement velocities. Each steady displacement velocity was taken to represent the "natural" speed of the sliding system corresponding to the applied friction coefficient.

Burwell and Rabinowicz claim that the initial and steady-state portions of their displacement $v s$ time curves represent, respectively, the primary and secondary stages of interfacial creep. Their experimental results are consistent with this explanation; as the applied friction coefficient increases, the length of the primary creep stage decreases, and the steady-state velocities increase. The positive slope of a crystalline material's friction-velocity curve thus represents the strain-rate sen- 


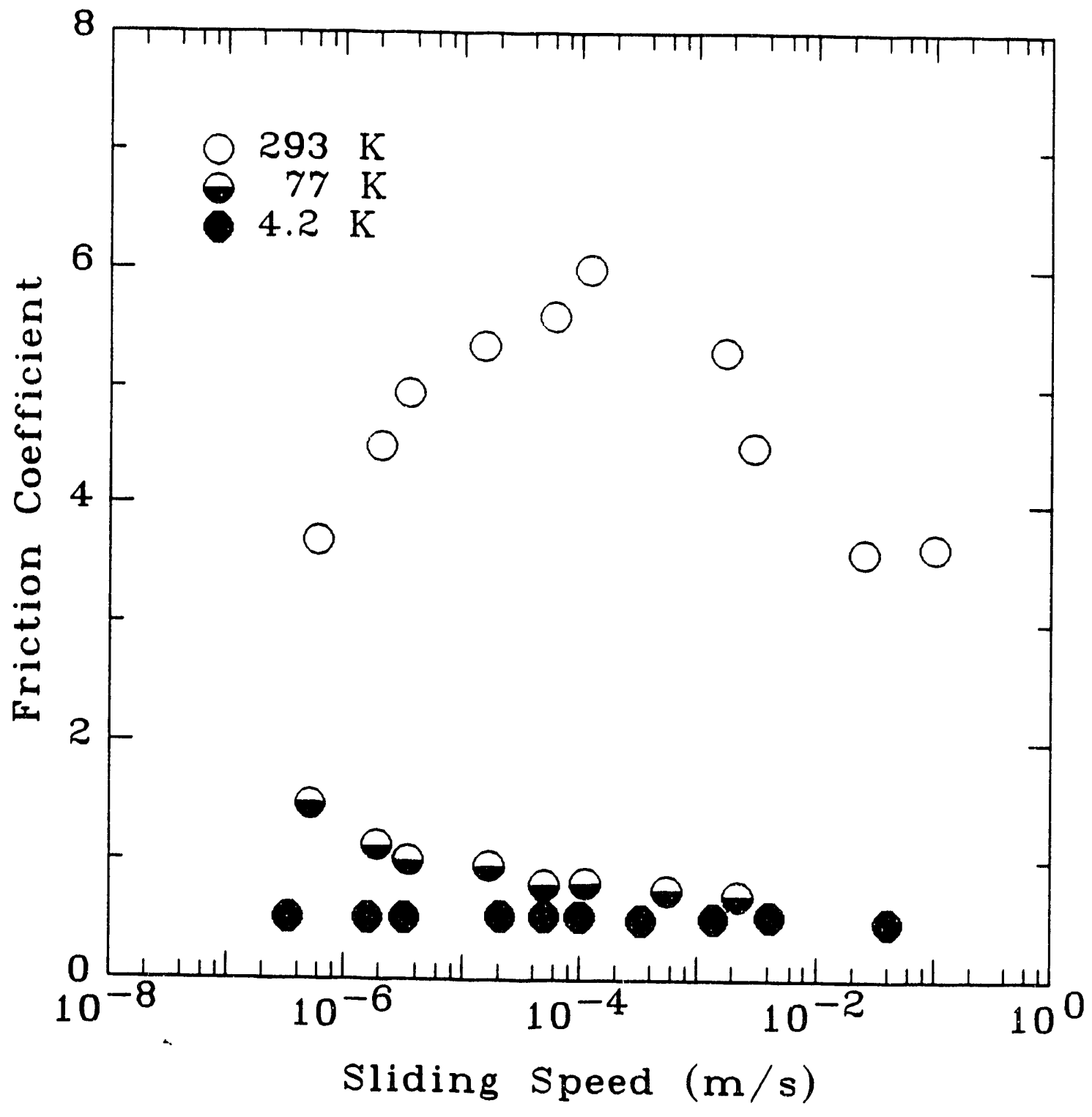

Figure 6.12: Friction coefficient vs sliding speed for an indium disk slid against AISI 316 stainless steel pins at $4.2,77$, and $293 \mathrm{~K}$. The normal force was $7.5 \mathrm{~N}$. 
sitivity of the junction creep stress in shear. ${ }^{[13]}$ Tertiary creep, leading to junction rupture, is not observed to the left of the friction-velocity peak because, in the sliding mode, material is continuously supplied at the leading edges of the asperities to make up for that lost along the trailing edges.

\subsubsection{Negative friction-velocity characteristics}

Equilibrium creep conditions comparable with those attained during Burwell and Rabinowicz's experiments are not generally achieved during conventional sliding experiments.

The development of steady-state creep is usually interpreted as a balance between strain hardening and thermal recovery. ${ }^{[30,31]}$ By contrast, primary creep constitutes a strain-hardening imbalance in which the rising dislocation density during deformation produces a corresponding decrease in the overall deformation rate. The thermally activated migration of vacancies through a crystalline material leads to thermal recovery by reducing the overall dislocation density.

As the sliding speed increases during a conventional friction experiment, less time is available for thermally-activated vacancy migration, hence a point is eventually reached where the shear deformation passes from creep to a state of plastic flow; this point typically occurs near the peak in the friction-velocity curve. According to Burwell and Rabinowicz the descending portion of the friction-velocity curve is caused by a velocity-dependent decrease in the effective interfacial contact area. Increasing sliding speeds to the right of the friction peak reduce the corresponding asperity contact time and change the junctions' shear deformation mode from ductile fracture to brittle failure. ${ }^{[13]}$

\subsubsection{Time-temperature superposition in crystalline solids}

The most disappointing aspect of the creep theory of friction is that it does not presently allow for the extrapolation of friction-velocity data from one test temperature to another. There are several reasons for this poor correlation. For one, the shear strains produced during metallic friction are usually confined to a thin ( $\sim 100 \mu \mathrm{m}$ deep) surface layer; the mechanical properties of these near surface layers are often markedly different from the crystal's bulk mechanical properties. ${ }^{[5,32]}$ Secondly, because of the large incremental strains produced during each asperity 
encounter the junction's flow properties can change markedly from the inception of the asperity contact, its growth to full strength, and subsequent separation. Third, the existence of broad transitions between a metal's deformation-rate and temperature-dependent flow properties greatly limits the viability of a single friction model for describing the whole range of possible frictional behaviors. The structural mechanics background necessary for analyzing these non-equilibrium, large-strain, near-surface-layer deformations is not sufficient developed to enable a direct solution for predicting the desired friction-velocity-temperature sliding characteristics. ${ }^{[5]}$

\subsubsection{Thermally activated fatty-acid friction}

Experimental evidence in support of thermally activated friction-velocitytemperature sliding has recently been observed in fatty-acid-lubricated copper-oncopper sliding pairs. The tests were performed using the pin-on-disk apparatus described in $\S 4.2 .3 .1$.

Figure 6.13a shows friction vs temperature traces for a lauric acid $\left(\mathrm{C}_{11} \mathrm{H}_{23} \mathrm{COOH}\right)$ lubricated copper-copper pair. The normal load was $7.5 \mathrm{~N}$ and the sliding speeds were $4 \times 10^{-4}, 4 \times 10^{-3}$, and $4 \times 10^{-2} \mathrm{~m} / \mathrm{s}$. The temperature during each tests was reduced at a rate of $\sim 50 \mathrm{~K} / \mathrm{hr}$ via convective heat transfer with the boil-off gas produced by transferring liquid nitrogen into the apparatus cryostat. Each friction coefficient represents the average value of 60 readings taken one second apart. These readings were taken at $20 \mathrm{~K}$ increments during the cooling curve. The friction coefficient variation for each data point was typically within \pm 0.01 . The test temperatures were determined to within $\pm 1 \mathrm{~K}$ using a sixth-order curve fit of the thermoelectric voltage provided by a constantan-chromel thermal couple imbedded in the copper disk specimen.

All three friction-temperatures traces show a marked change in their friction coefficients from values near 0.4 below $\sim 160 \mathrm{~K}$ to values around 0.1 near room temperature. Similar frictional variations have also been observed during during boundary-lubricated studies at the Wright-Patterson Air Force Base. ${ }^{[33,34]}$

Organic solid lubricants typically consist of a single, low- to moderate-molecularweight non-polar polymer chain terminated by a polar end group. The polar end group allows the molecules to condense onto a metal surface into a densely-packed 

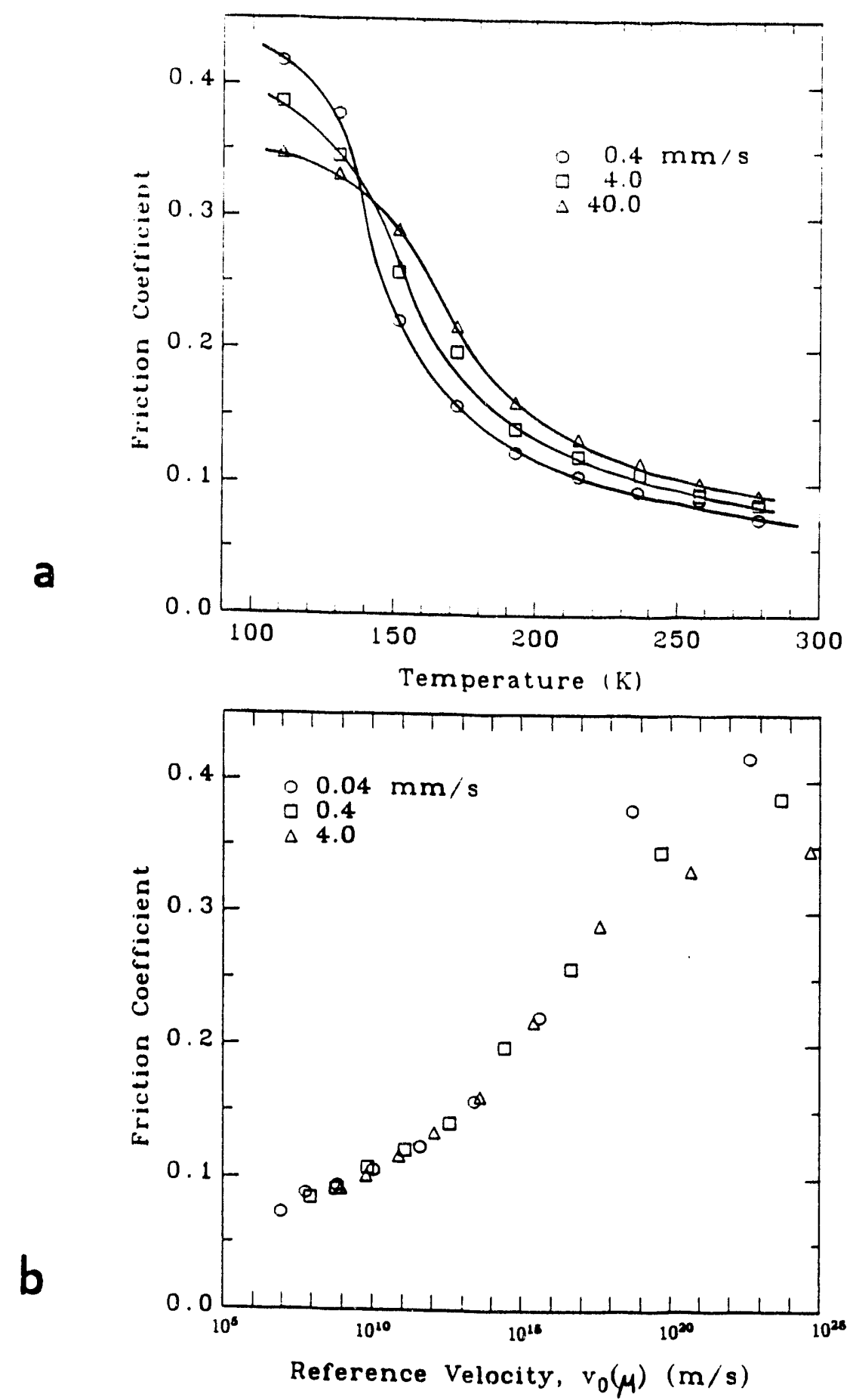

Figure 6.13: Friction-velocity-temperature correlation for lauric-acid-lubricated copper-copper sliding pair. a) Friction vs temperature traces for sliding speeds of $4 \times 10^{-4}, 4 \times 10^{-3}$, and $4 \times 10^{-2} \mathrm{~m} \mathrm{~s}^{-1}$. b) Lauric-acid friction master curve obtained by applying thermally-activated regression to data in Figure 6.13a. 
array corresponding to the lubricant's normal crystalline packing arrangement. The lubricant remains effective as long as minimum, monomolecular coverage is maintained. [35]

The relatively uniform spacing between the individual friction-temperature traces in Figure 6.13a suggests the probable existence of a single lauric-acidlubricated-copper master curve. This possibility is explored using the following thermally-activated model. ${ }^{[36]}$

$$
v(\mu, T)=v_{0}(\mu) \exp \left(\frac{E_{a}}{k T}\right)
$$

where $\mu$ is the friction coefficient, $T$ the temperature, and $v(\mu, T)$ the sliding velocity used to derive each friction-temperature trace. $v_{0}(\mu)$ is a reference velocity determined by the data correlation, and $E_{a}$ is the activation energy of the frictional relaxation.

The activation energy was obtained in the usual manner, by cross-plotting the friction-temperature data as a series of $\log (v(\mu, T))$ vs $1 / T$ lines. [37] Four friction values were used to construct this crossplot; they were $\mu=0.15,0.20,0.25$, and 0.10 . The slopes of these lines were constant to within $2 \%$ of each other and indicate an average activation energy of $E_{a}=13.2 \mathrm{kcal} / \mathrm{mole}$.

Figure $6.13 \mathrm{~b}$ plots the results of this thermally-activated regression by replotting the lauric-acid friction data as a single $\mu$ vs $v_{0}(\mu)$ master curve, where

$$
v_{0}(\mu)=v(\mu, T) \exp \left(\frac{-E_{a}}{k T}\right) .
$$

The good fit of the data to a single curve indicates the viability of this thermallyactivated mechanism for characterizing the frictional characteristics of crystalline materials. The velocity-dependent variation in the peak friction values (Figure 6.13a) causes the divergence observed in the high-speed portion of the master curve (Figure 6.13b). Friction-velocity-temperature master curves have also been obtained for stearic- and octanoic-acid lubricated surfaces.

\subsubsection{Mechanical relaxations in fatty-acid friction}

I believe, but have not yet been able to prove, that the $\sim 150$-K frictional relaxation for the fatty-acid-lubricated copper surfaces is related to a mechanical 
relaxation that occurs in virtually all polymers possessing three of more adjacent $\left(-\mathrm{CH}_{2-}\right)$ segments along their backbone chains. ${ }^{[38]}$ Partial evidence in support of this contention is provided in Figure 6.14, which shows the friction vs temperature curves for three members of the homologous alkanoic fatty acids series, namely, octanoic $\left(\mathrm{C}_{7} \mathrm{H}_{15} \mathrm{COOH}\right)$, lauric $\left(\mathrm{C}_{11} \mathrm{H}_{23} \mathrm{COOH}\right)$, and stearic $\left(\mathrm{C}_{17} \mathrm{H}_{35} \mathrm{COOH}\right)$ acid.

The friction-temperature traces were collected by first cooling the pin-on-disk apparatus to liquid helium temperatures. Sliding was then initiated at a speed of $3 \times 10^{-5} \mathrm{~m} / \mathrm{s}$. The data during each test was recorded continuously as the friction apparatus warmed to room temperature via conduction down the apparatus support tube. The normal force was $7.5 \mathrm{~N}$ and the warming rate was approximately $\sim 5 \mathrm{~K} / \mathrm{hr}$.

All acids demonstrate a pronounced peak in their friction-temperature traces in the range $100 \sim 160 \mathrm{~K}$ before settling to friction values in the range $0.1 \sim 0.15$ near room temperature. In addition, and perhaps more importantly, the temperature corresponding to the peak friction value shifts to the right with increasing chain length in proportion to the increase in the acid's respective melting temperature. For the sliding velocity used, the ratio between the peak-friction temperature and the melting temperature assumes a constant value of $\sim 0.4$.

The existence of a second friction-temperature peak in the $0 \sim 50 \mathrm{~K}$ temperature range is of considerable interest from a magnet-technology standpoint, in that it suggests a non-negligible, cryogenic-temperature, friction-velocity behavior. Figure 6.15 presents a closer examination of the friction $v s$ velocity characteristics for stearic-acid-lubricated copper specimens at 4.2 and $293 \mathrm{~K}$. The friction-velocity curve at $4.2 \mathrm{~K}$ shows a relatively high friction value of $\sim 0.40$ and a slightly negative friction-velocity slope. By contrast, the $293-\mathrm{K}$ friction values are closer to 0.1 and demonstrate slightly positive friction-velocity characteristics. These results are consistent with the general friction $v s$ temperature behaviors discussed in $\$ 6.2 .4$. Friction coefficients to the left of a mechanical relaxation peak typically demonstrate negative friction-velocity tendencies whereas friction coefficients to the right of the peak show positive friction-velocity characteristics.

It should be noted that results for these low-shear-strength boundary lubricants cannot readily be extrapolated to the general circumstances of unlubricated solid 


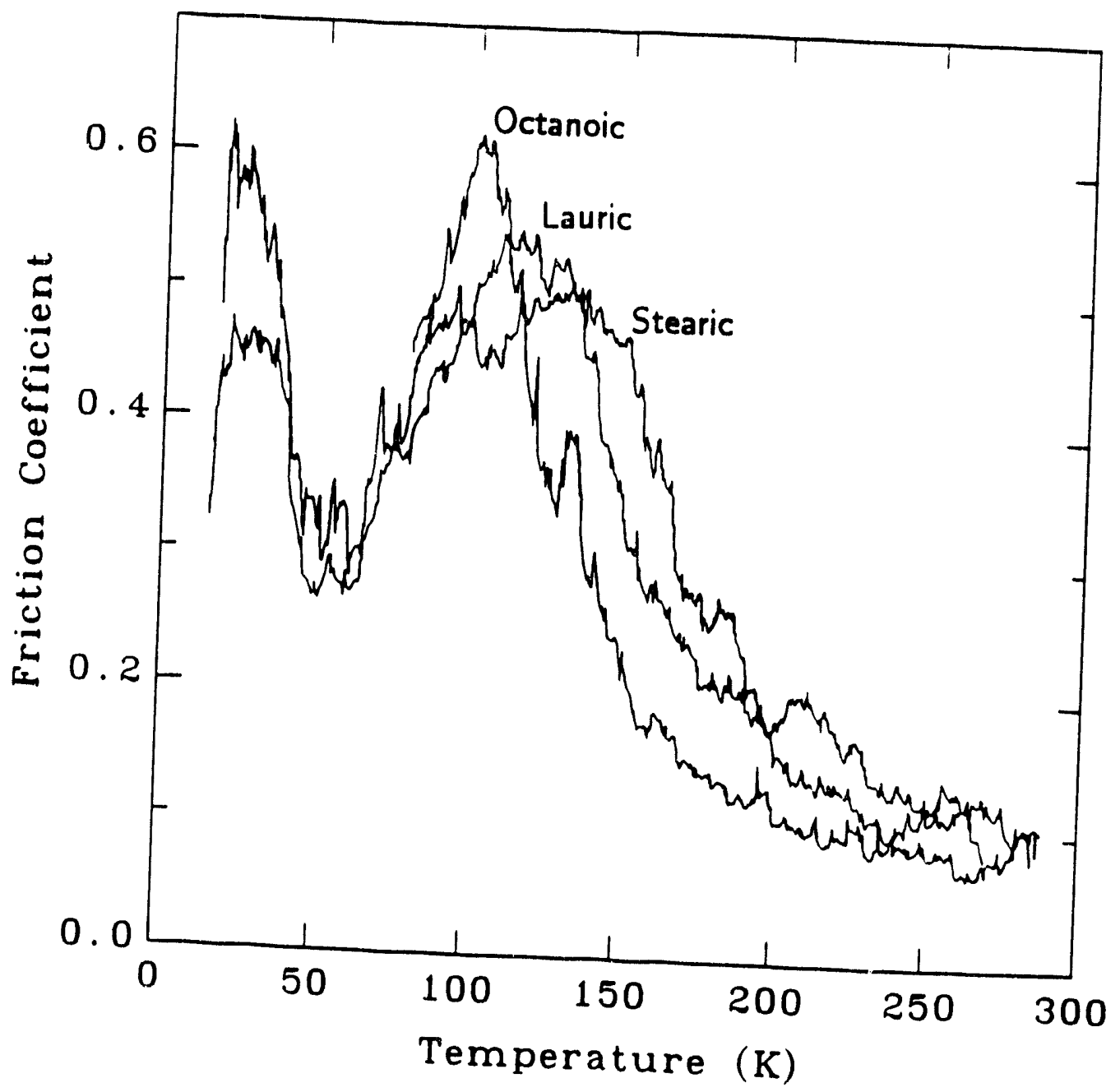

Figure 6.14: Friction vs temperature traces for octanoic-, lauric-, and stearicacid-lubricated copper-copper pairs. The sliding speed was $4 \times 10^{-4} \mathrm{~m} \mathrm{~s}^{-1}$ and the normal load was $7.5 \mathrm{~N}$. 


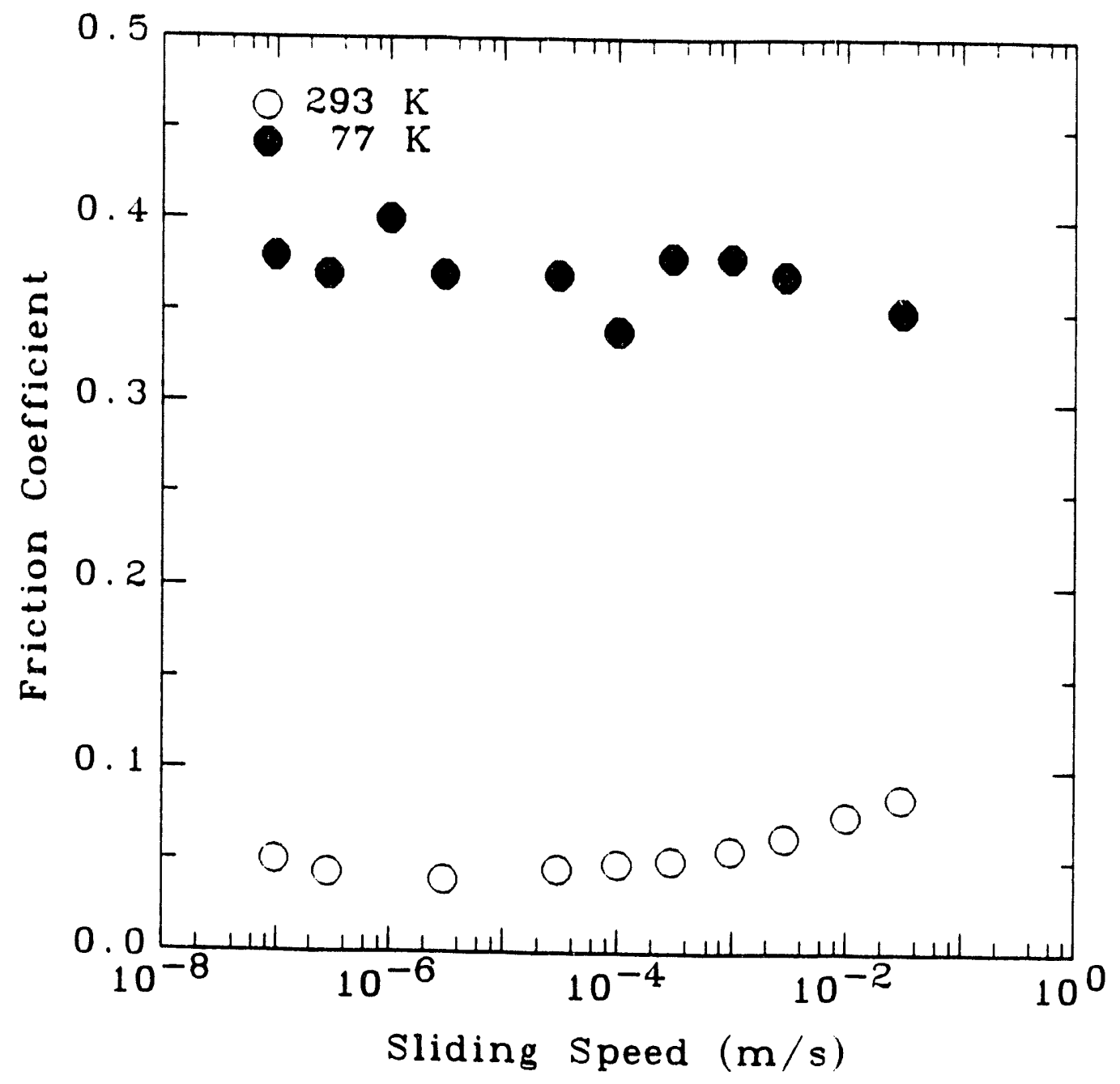

Figure 6.15: Friction $v s$ velocity traces for stearic-acid-lubricated copper at 4.2 and $293 \mathrm{~K}$. 
friction. The use of a very thin, low-shear-strength coating on a much harder substrate effectively decouples the velocity- and temperature-dependent shear-strength component, $s_{l}$, of the friction coefficient from the velocity- and temperaturedependent area component, $P$. This effect is demonstrated by Eq. 4.4

$$
\mu_{1}=\alpha \frac{s_{1}}{P}+(1-\alpha) \frac{s}{P}
$$

assuming a very effective $(\alpha \simeq 1)$ lubricant coverage. Very good lubricant coverage by the fatty acids throughout the cryogenic temperature region has been confirmed through the use of contact resistance measurements. ${ }^{[33,94]}$ By contrast, the interplay between the normal- and shear-stress components for single phase materials is typically modeled as an equivalent plastic flow phenomena, characterized for instance by the von Mises or Tresca criterion. ${ }^{[3,5,6,13]}$

\subsubsection{Metallic, friction-temperature effects}

The three general friction-velocity-temperature behaviors presented in Figure 6.12 are marked by similar transitions in friction-temperature behavior. Figure 6.13 presents friction vs temperature data for an indium disk slid against three AISI 316 stainless steel pins at a fixed sliding speed of $2 \times 10^{-6} \mathrm{~m} / \mathrm{s}$.

The friction-temperature data were collected using the pin-on-disk friction apparatus. The mercury disk was prepared by filling a annular brass trough with room temperature mercury, inserting and carefully leveling both specimen and apparatus into the test cryostat, and slowly cooling the samples to liquid helium temperatures via convective heat transfer with the boil-off gas produced by transferring liquid helium into the cryostat. The data was recorded continuously as the mercury disk warmed from $\sim 5$ to its $235 \mathrm{~K}$ melting temperature.

Figure 6.16 shows two fairly prominent friction-temperature transitions. During the low- to moderate-temperature transition the friction coefficient passes from a low, velocity-independent value to a much higher value, characterized by negative friction-velocity slope. Similar results were observed for indium, tin, lead, and copper specimens. This low-temperature transition is also reported by Bowden and Childs for the face-centered-cubic (fcc) metals: gold, silver, copper and nickel, and for the body-centered-cubic (bcc) metals: tantalum and tungsten. ${ }^{\text {[29] }}$ Bowden and Childs attribute this transition to the marked decrease in each fcc metal's initial 


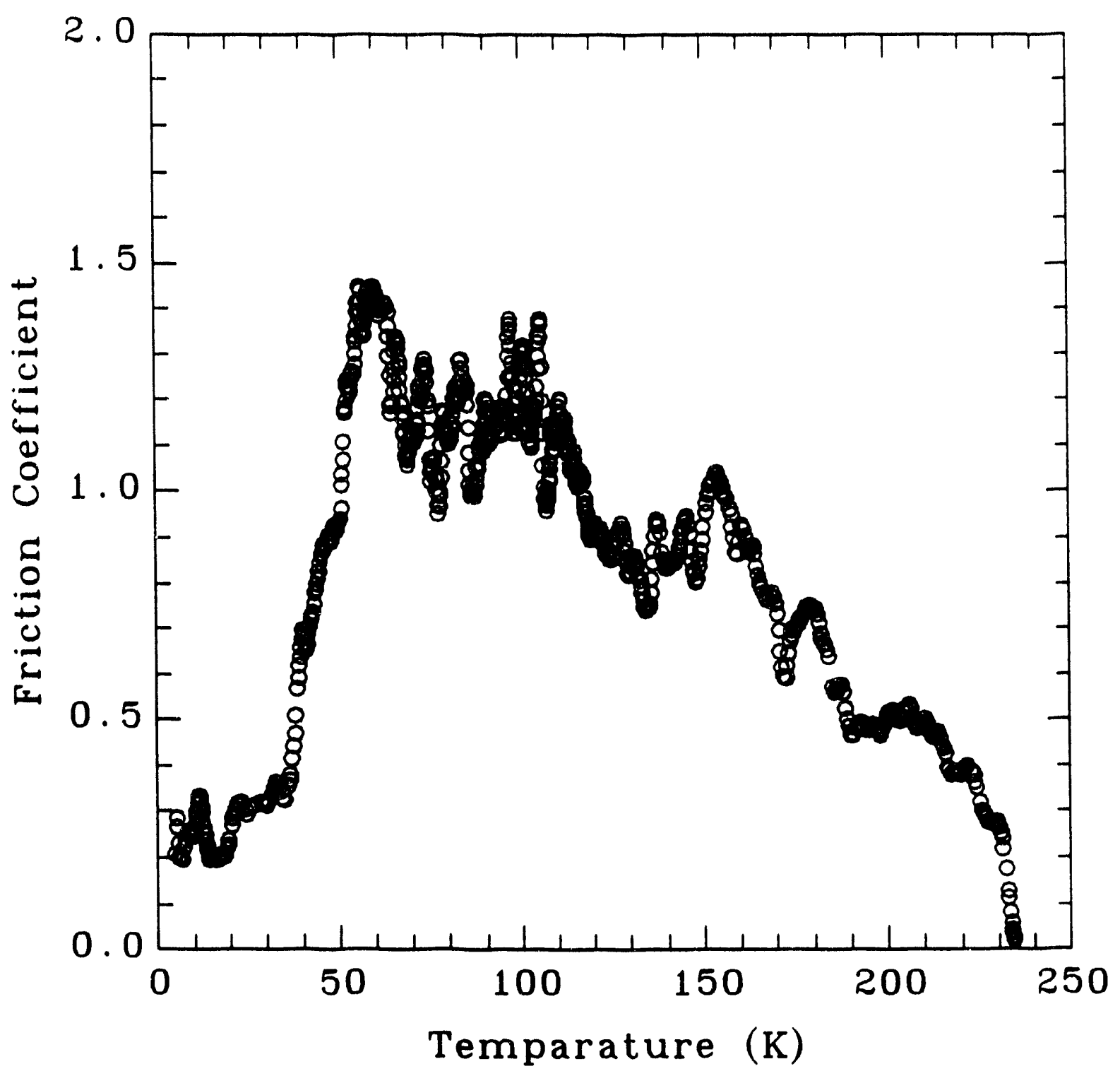

Figure 6.16: Friction $v s$ temperature trace for a mercury disk slid against AISI 316 stainless steel. The sliding speed was $2 \times 10^{-6} \mathrm{~m} \mathrm{~s}^{-1}$, and the normal load was $7.5 \mathrm{~N}$. 
strain hardening rates, $\delta \sigma / \delta \dot{\varepsilon}$ above the metal's frictional transition temperatures. This contention is consistent with Greenwood and Tabor who note that less-highlywork-hardened junctions persist over much larger sliding displacements and dissipate considerably more energy than saturation-work-hardened junctions. ${ }^{[4]}$ Thus, the increase in the friction coefficient at moderate temperatures is tentatively attributed to a change in the junctions' shear deformation mode from brittle to ductile fracture.

Coffin investigated the reversibility of this low- to moderate-temperature friction transition for copper-copper pairs. ${ }^{[28]}$ He observed that although the transition temperature is highly repeatable from one temperature cycle to the next, any increase in speed produces a marked increase in the corresponding transition temperature. The existence of this velocity-dependent transition temperature is consistent with the development of negative friction-velocity characteristics in the intermediatetemperature region.

Reports of a second, moderate- to high-temperature transition from high- to low-friction values have also appeared in the tribological literature. ${ }^{[27,39]}$ The hightemperature transition is attributed to thermal softening that occurs in a specimen's near-surface layers as the sliding temperature approaches the material's melting temperature temperature. Reduced friction coefficients are observed when the interfacial shear strength decreases more rapidly with temperature than the junction's penetration hardness. ${ }^{[27]}$

The friction-temperature-velocity characteristics in Figs. 6.12 and 6.16 have an approximate counterpart in the metals' time- and temperature-dependent hardness characteristics. Many investigators report the existence of two distinct inflections in hardness $v s$ temperature data as a general characteristic of crystalline materials. ${ }^{[40-42]}$ The ratio of these inflection temperatures to the crystal's melting temperature correspond roughly to the friction-temperature transitions observed in Figure 6.16. The first inflection temperature occurs near $15 \sim 20 \%$ of the melting temperature and the second in the vicinity of $50 \sim 60 \%$ of $T_{n 2}$. The use of hardness measurements as an approximate guide to frictional behavior was previously discussed in $\S 6.2 .6$. 
Mulhearn and Tabor provide a detailed record of time- and temperaturedependent hardness measurements for the low-melting-point metals indium and lead. ${ }^{[43]}$ Their data suggest the existence of of three distinct time- and temperaturedependent deformation mechanisms. At temperatures below $\sim 20 \%$ of the melting temperature, $T_{m}$, they found that their metallic hardness values were relatively high and independent of time under load. Friction in this temperature range (Figure 6.12) is also low and generally independent of velocity.

At temperatures above $\sim 60 \%$ of $T_{m}$, Mulhearn and Tabor report that their hardness values decreased linearly with loading time when plotted on a log-log scale. They analyzed the temperature separations between these $\log$ (hardness)-log(time) traces using an Arrhenius-type deformation-rate equation and found that the activation energy for the decrease in hardness was virtually identical to each metal's self-diffusion activation energy. As noted in $\S 6.3 .1 .1$, positive friction-velocity characteristics are generally observed only in this high-temperature deformation region. In the intermediate temperature region, the hardness values shows a time-dependent behavior suggestive of transient creep; the hardness values asymptotically approach fixed values at very long loading times.

It is generally conceded that the plastic deformation that occurs during metallic friction is conveyed on the dislocation level $;^{[5,44]}$ this accounts for the close similarity between a metal's friction-velocity-temperature characteristics and its time- and temperature-dependent hardness values. The principal obstacle to the continued deformation of a pure metals is caused by the interaction of moving dislocations on intersecting slip systems. ${ }^{[30,31]}$ As deformation continues the number of dislocation interactions increases thus raising the plastic flow stress. This rapid rise in dislocation interactions is responsible for increase in the fcc metals' initial low-temperature strain-hardening rate.

As the deformation temperature passes above $\sim 25 \%$ of the melting temperature, thermal activation becomes increasingly effective at easing the moving dislocations past one another; this transition is typically marked by a reduced strain hardening rate and greatly increased ductility. ${ }^{[30]} \mathrm{A}$ second transition is observed in the plastic flow behavior above $50 \sim 60 \%$ of $T_{m}$ at which point the thermally-activated diffusion of vacancies through the crystal becomes increasing effective at reducing its overall 
dislocation density. ${ }^{[30,44]}$

This interpretation of metallic friction in terms of dislocation dynamics is tenuous at best, in that it greatly oversimplifies the complex states of stress and strain that exist at an asperity junction during sliding. However, the similarities between the metals' bulk mechanical properties, such as hardness, and their friction-velocity characteristics are similar enough to suggest that hardness data can be used as an approximate guide for selecting low-temperature friction materials. This materialselection guide likewise indicates that at best constant friction coefficients are the most stable frictional-velocity characteristic that could be hoped for at liquid-helium temperatures.

\subsection{Conductor stiffness modelling}

The preceeding review of cryogenic-temperature friction material-selection guidelines indicates that the choice of sliding materials alone is insufficient for stabilizing abrupt conductor motions. Fortunately, the stick-slip model in $\$ 6.1$ suggests that the unstable slips can also be moderated by increasing the sliding system stiffness. Several conductor stifiness models are proposed as a means of developing a structural approach towards dry-wound magnet stabilization.

Unstable conductor motions can exist on a varieity of dimensional scales. The smallest involve conductor segment lengths shorter than a single conductor turn. Intermediate-sized motions involve the displacement of entire conductor turns. Global motions are also possible; these are produced by the elastic deformation of the entire winding body and lead to frictional heating along the winding/supportstructure interface.

Three conductor motion stiffness models will be developed; one for each of the dimensional scales listed above. Unstable slips are possible when a model's combined system stiffness, $k+k_{j}$, can no longer maintain the system's corresponding electromagnetic displacement, $\delta_{z}$, to below an asperity contact diameter, that is, slip is assumed to occur when $\delta_{z}$ exceeds $\sim 5 \mu \mathrm{m}$.

The conductor stiffness models will be pursued with respect to a hypothetical small winding model described in Table 6.1. This winding model combines representative feature from Urata and Maeda's test magnet ${ }^{[2 \theta]}$ with those of the test coil 
implemented in Ch. 7. Table 6.1 lists the winding model's electromagnetic characteristics, its frictional properties, approximate overall stress state, and typical mechanical and physical properties for small rectangularly-cross-sectioned conductors. The models presume that motions occur most frequently near the coil's ends, thus, the axial and radial magnetic inductions necessary for the electromagnetic force calculations at this coil location are included in Table 6.1.

Table 6.1: Magnet Parameters for Conductor-Stiffness Model.

\begin{tabular}{|l|c|}
\hline Winding diameter, $2 a_{1}(\mathrm{~mm})$ & 80.0 \\
\hline Coil length, $2 b(\mathrm{~mm})$ & 100.0 \\
\hline Operating current, $I_{o p}(\mathrm{~A})$ & 500.0 \\
\hline Central induction, $B_{0}(\mathrm{~T})$ & $7 \sim 8$ \\
\hline Radial induction at coil end, $B_{r}(\mathrm{~T})$ & $\sim 3$ \\
\hline Axial induction at coil end, $B_{z}(\mathrm{~T})$ & $\sim 5$ \\
\hline Radial stress, $\sigma_{r}(\mathrm{MPa})$ & $0.5 \sim 2.5$ \\
\hline Static friction coefficient, $\mu_{s}$ & 0.26 \\
\hline Asperity junction diameter, $\delta_{z}(\mu \mathrm{m})$ & $\sim 5$ \\
\hline Conductor width, $w(\mathrm{~mm})$ & 1.3 \\
\hline Conductor thickness, $t(\mathrm{~mm})$ & 1.0 \\
\hline Radial modulus, $E_{r}(\mathrm{GPa})$ & 60.0 \\
\hline Hoop modulus, $E_{\theta}(\mathrm{GPa})$ & 90.0 \\
\hline Axial modulus, $E_{z}(\mathrm{GPa})$ & 70.0 \\
\hline 300-4.2 K axial thermal contraction, $\Delta_{z}$ & 0.003 \\
\hline
\end{tabular}

All directional subscripts in the analyses are referenced to the coil coordinates. For instance, the conductor axis lies along the winding's circumferential, $\theta$, direction. The frictional normal force is provided by the coil's radial stress component, $\sigma_{r}$, and conductor motions occur in the coil's axial, $z$, direction.

\subsubsection{Short conductor segrisent slips}

The axial electromagnetic body force on the conductors in a solenoid are greatest near the ends of the magnet where the radial component of the magnetic induction is also high. Thus, the axial friction force per unit conductor length, $f_{z}$, increases during energization and attains a final value of:

$$
f_{z}=I_{o p} \times B_{r}
$$


where $I_{o p}$ is the coil's designated operating current and $B_{r}$ is the radial induction at the coil ends. The maximum value of $f_{z}$ for the model coil is $1500 \mathrm{~N} / \mathrm{m}$.

A common approached for preventing conductor motions in dry-wound coils is to apply sufficient conductor tension during manufacture so that the resulting radial precompression raises the static friction force between the conductor layers to a value greater than the applied axial electromagnetic force. The minimum radial contact force per unit length, $f_{r}$, needed to raise the conductor's static friction force to the level of its axial electromagnetic force is given by:

$$
2 f_{r}=\frac{f_{z}}{\mu_{s}}
$$

The factor of two appears in Eq. 6.18 because the same radial force is applied to both conductor faces. This contact force can also be converted to an equivalent radial pressure, $\sigma_{\tau}$ by dividing through by the conductor width, $w$.

$$
\sigma_{r}=\frac{f_{r}}{w}=\frac{f_{z}}{2 \mu_{s} w}
$$

For our theoretical winding model, $\sigma_{r}$ would have to be greater than $2.2 \mathrm{MPa}$ to prevent axial wire motions.

Equation 6.19 assumes, of course, that the conductor has a perfectly uniform cross-section. By contrast, the dimensional tolerances for a high-quality conductor typically averages around $\pm 2 \mu \mathrm{m}$. ${ }^{[45]}$ When the $1 \mathrm{~mm}$ thick model conductor from Table 6.1 is subjected to $\sigma_{r}=2.2 \mathrm{MPa}$, its corresponding radial elastic deformation is $\sim 0.04 \mu \mathrm{m}$. Because the conductor's radial deformation is much smaller than its dimensional tolerance certain conductor segment lengths will invariably be held less tightly than Eq. 6.19 indicates; this is not to say that these conductor segments will not be subject to any radial pressure, but rather that the segments' actual radial pressures will be less than the predicted nominal contact pressure. The offset between the assumed and nominal contact pressure accounts for the persistence of conductor motion disturbances in tightly wound magnets, where such disturbances are otherwise assumed to be impossible. ${ }^{[28]}$

Figure 6.17 shows the conductor-segment model used to determine the effective stiffness of a poorly-supported conductor segments length. The radial contact force 
per length, $f_{r}^{\prime}$, for the poorly supported segment is produced by the segment's radial electromagnetic body force.

$$
f_{r}^{\prime}=I_{o p} \times B_{z}
$$

where $B_{z}$ is the coil's axial magnetic induction. The segment is modelled as a cantilevered beam of length $2 \ell$ that is rigidly clamped at both ends and subjected to a uniform load intensity, $\left(f_{z}-\mu_{s} f_{r}^{\prime}\right)$, determined by the difference between the segment's axial electromagnetic force and its static friction force.

Tsukamoto, Takao and Honjoh ${ }^{[4 \theta]}$ performed a similar analysis of this arrangement for cryostable magnets and report the following equation for the segment length's central deflection, $\delta_{z}$.

$$
\delta_{z}=\frac{\left(f_{z}-\mu_{\theta} f_{r}^{\prime}\right) \ell^{4}}{24 E_{\theta} J_{r}}
$$

where $E_{\theta}$, is the conductor's bending modulus, and $J_{r}$ its bending moment of inertia. Equation 6.21 can be used to determine the minimum, poorly-supported conductor segment length capable of unstable conductor motions. Substituting in the values: $\delta_{z}=5 \mu \mathrm{m}, f_{z}=1500 \mathrm{~N} / \mathrm{m}, \mu_{s}=0.26, f_{r}^{\prime}=2500 \mathrm{~N} / \mathrm{m}, E_{\theta}=90 \mathrm{GPa}$, and $J_{r}=t w^{3} / 12=0.183 \mathrm{~mm}^{4}$, yields $2 \ell=14 \mathrm{~mm}$. This $14 \mathrm{~mm}$ conductor length is somewhat smaller than, but of the same order of magnitude as, the $20 \sim 30 \mathrm{~mm}$ unstable segment lengths reported by Maeda during conductor motion experiments using slightly smaller diameter conductors. ${ }^{[4]}$

\subsubsection{Whole turn conductor stiffness}

The lower radial contact pressures in less tightly wound magnets allows much longer conductor segment to participate in conductor motion disturbances. A model is developed to examine motions involving entire conductor turns for an assumed radial contact pressure of $0.5 \mathrm{MPa}$.

Resistance to whole turn conductor motions is provided both by interlayer friction and by the winding acting as a tightly-coiled spring. Crandall, Dahl, and Lardner propose the following expression for the axial spring constant, $k_{z}$, for the single turn of a coil spring: ${ }^{[48]}$

$$
k_{z}=\frac{G_{\theta} J_{\theta}}{2 \pi a_{1}^{3}}
$$



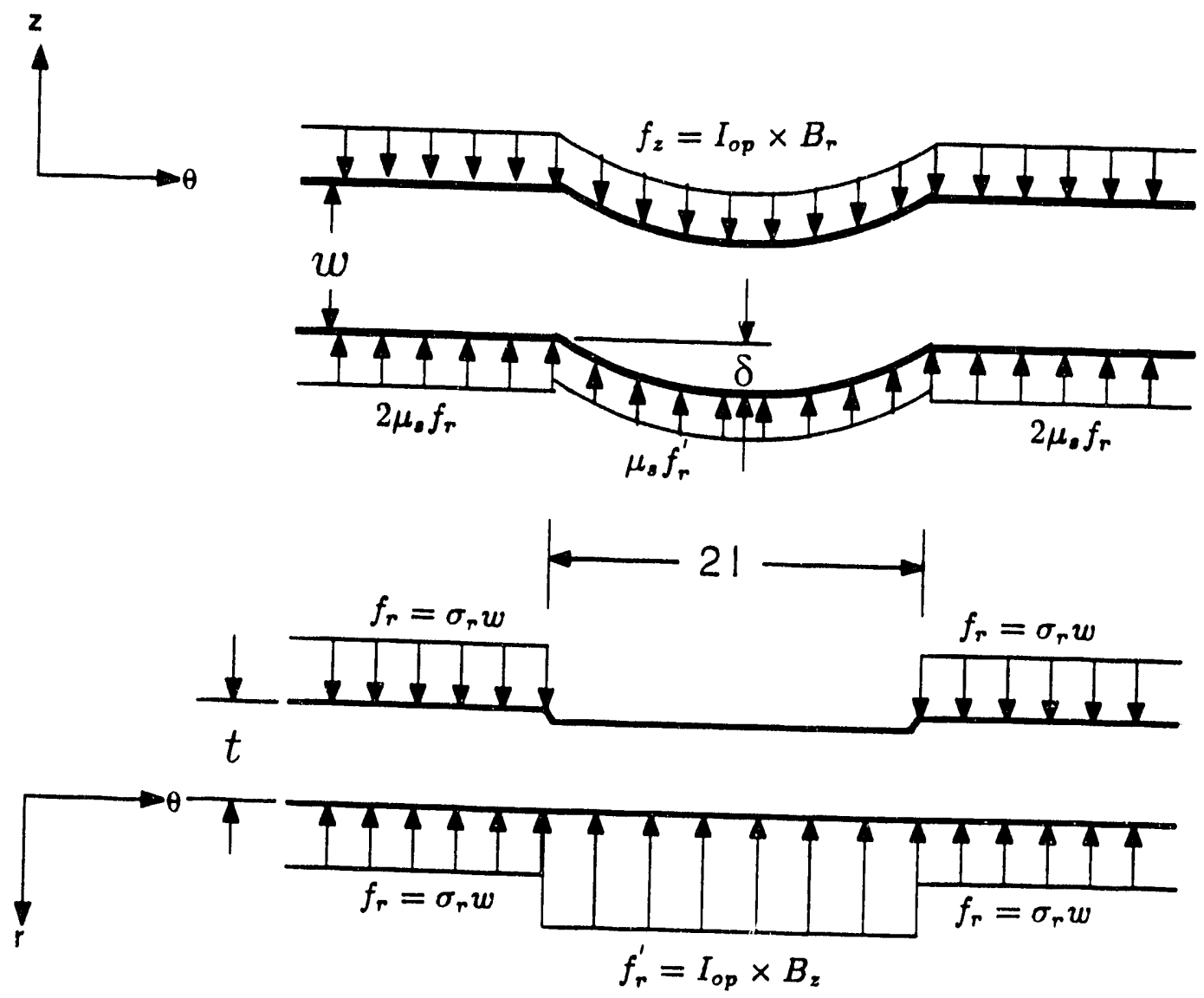

Figure 6.17: Stiffness model for short conductor segments. Variations in the conductor thickness produce segment lengths, $2 \ell$, that are less well supported than theoretically assumed. The stiffness of the poorly-supported segment is determined by modelling the segment as a cantilevered beam subjected to a uniform distributed load, $\left(f_{z}-\mu_{s} f_{r}^{\prime}\right)$. 
where $G_{\theta}$ is the conductor's torsional modulus, $J_{\theta}$ the torsional moment of inertia, and $a_{1}$ the winding's inner radius. An approximate value for $G_{\theta}=35 \mathrm{GPa}$ can be obtained by applying the isotropic relation that $G \sim E / 2.6$ to the model conductor. $J_{\theta}$ for a rectangular prismatic shaft is given by ${ }^{[48 !}$

$$
J_{\theta}=c_{2} t w^{3}
$$

where $c_{2}$ is determined by the conductor's aspect ratio, which for our model conductor is $\sim 0.158$. Substituting the conductor properties and winding geometry into Eq. 6.22 yields an effective single turn axial stiffness of $k_{z}=30 \mathrm{~N} / \mathrm{m}$.

This effective axial stiffness can now be used to determine the minimum charging current, $I$, for which an unstable whole-turn conductor slip can occur.

$$
\begin{aligned}
k_{z} \delta_{z} & \approx 2 \pi a_{1}\left(f_{z}-\mu_{s} f_{r}\right) \\
k_{z} \delta_{z} & \approx 2 \pi a_{1}\left(I^{2} \frac{B_{r}}{I_{o p}}-2 \mu_{s} \sigma_{r} w\right) \\
I^{2} & \approx \frac{\left(\frac{k_{z} \delta_{z}}{2 \pi a_{1}}+2 \mu_{s} \sigma_{r} w\right) I_{o p}}{B_{r}}
\end{aligned}
$$

For a radial contact pressure of $0.5 \mathrm{MPa}$ the conductor turn at the end of the coil slips $\left(\delta_{z}>5 \mu \mathrm{m}\right)$ when the charging current passes above $\sim 208 \mathrm{~A}$. Equation 6.24 is an approximate expression because, whereas Eq. 6.23 is derived with the load applied only to the ends of the turn, the corresponding force in the magnet is uniformly distributed along the conductor's length. A comparison of the conductor's static friction force to its axial spring force at the point of slip shows that the friction force is by far the largest obstacle to motion in this loosely-wound winding model.

$$
4 \pi a_{1} \mu_{,} \sigma_{r} w \gg k_{z} \delta_{z}
$$

As the charging current increases, the imbalance between the axial electromagnetic force and the static friction force continues to rise and the simultaneous motion of several adjacent conductor turns becomes more and more common. Fortunately, because the radial magnetic induction decreases towards the solenoid's midplane, a point is eventually reached where the net friction on the accumulated conductor turns just balances the cumulative axial electromagnetic force. 
For very long coils, the radial magnetic induction remains essentially zero over much of the coil's length and increases rapidly near the coil's ends to a substantial fraction of its central magnetic induction. For relatively short coils, like the one in this example, the radial induction is negligible over the central half of the winding and increases more of less linearly from there towards the coil's ends. The following approximate variation will be used to determine the point at which the cumulative conductor motions stop.

$$
B_{r}(z)= \begin{cases}0, & \text { for } 0<|z|<\frac{b}{2} \\ \frac{2 B_{r}}{b}\left(z-\frac{b}{2}\right) & \text { for } \frac{b}{2}<|z|<b\end{cases}
$$

where $b$ is the coil's half-length.

Conductor motions in our analytic, loose-wound coil model should cease when the cumulative electromagnetic force on the conductors just balances the cumulative static friction force

$$
\int_{z}^{b}\left(f_{z}(z)-2 \mu, \sigma_{r}\right) d z=0
$$

For the assumed coil parameters in this example

$$
\begin{array}{r}
\int_{0}^{b} f_{z}(z) d z=\int_{0}^{b} \frac{I_{o P}}{w} B_{r}(z) d z=14,425 \mathrm{~N} / \mathrm{m} \\
\int_{0}^{b} 2 \mu_{,} \sigma_{r} d z=13,000 \mathrm{~N} / \mathrm{m}
\end{array}
$$

Equation 6.28 indicates that, at the coil's designated operating current, the net axial electromagnetic force exceeds the conductor's static friction forces. Hence, conductor motion in this loosely-wound coil will not stop until all of the interconductor gaps have been removed from the winding; at this point the difference between the electromagnetic forces and friction forces are converted into axial contact forces between the conductors. These axial forces vary from a maximum at the coil's midplane to near zero at the coil's ends. Similarly, because the static friction force is much greater than the axial stiffness of the conductor turns (Eq. 6.25) virtually none of the conductor's axial displacement is recovered upon removal of the electromagnetic forces. Both tendencies are observed in the conductor-motion-induced training behavior reported in \$5.3.3 for Urata and Maeda's loosely-wound coil. ${ }^{[2 \theta]}$ The conductor motion disturbances in their loosely-wound coils were frequently accompanied by very large voltage spikes, signifying the cumulative motion of several 
adjacent conductors. The occurrence of Kaiser-effect training to full critical current likewise indicates the elimination of the coil's interconductor gaps between charging cycles.

\subsubsection{Rigid body coil motions}

The mechanical behavior of a fully-axially-compacted dry-wound coil should be virtually identical to that of an impregnated winding. Hence, the problem of interfacial winding motion that was previously discussed in §3.2.2.1 with regard to impregnated magnets may also pose a threat to the operational stability of drywindings.

Because of the symmetry of its electromagnetic loading, the displacements in a solenoidal winding accumulate from zero at the midplane to maximum values near the coil ends. Unstable motions are most likely near the coil ends where the accumulated displacements are greatest. ${ }^{[49,50]}$ The axial stiffness, $k_{z}$ of an axiallycontinuous conductor layer is determined by the conductor's elastic modulus, $E_{z}$, and thickness, $t$, the coil half-length, $b$, and the layer's circumference, $2 \pi a_{1}$.

$$
\frac{k_{z}}{2 \pi a_{1}}=\frac{E_{z} t}{b}
$$

Thus, the axial displacement at the end of the analytic winding model can be determined by dividing the net axial body force by the effective winding stiffness

$$
\frac{2 \pi a_{1} \int_{0}^{b}\left(f_{z}(z)-\mu \sigma_{r}\right) d z}{k_{z}} \approx \delta
$$

where $\delta$ is the deflection at the end of the coil.

An approximate relation is again used because whereas Eq. 6.30 assumes that the net body force acts entirely at the end of the winding, the actual body forces are distributed along the winding's length. The friction force appears only once in Eq. 6.30 because there is only one interface between the coil form and winding. For an $0.5 \mathrm{MPa}$ radial contact pressure, Eq. 6.30 predicts a $5.7 \mu \mathrm{m}$ displacement at the coil's ends. This is just slightly larger than the assumed asperity contact diameter. Consequently, the coil should demonstrate marginal behavior with respect to interfacial winding motions. Equation 6.30 highlights two tendencies previously observed with regard to interfacial winding motions. The incidence of interfacial 
windiug motions tend to increase with increasing coil length, ${ }^{[50]}$ and with decreasing radial contact pressures. ${ }^{[4 \theta]}$

The most effective method for preventing interfacial-motion-induced quenches in impregnated solenoids is to allow the winding to entirely separate from its coil form at low charging currents $\left(\sigma_{r}\left(a_{1}\right)=0\right)$ and to attach low-thermal-conductivity friction blocks to the winding at any possible contact point with the coil form. ${ }^{[51]}$ These blocks thermally isolate the winding from potential frictional heating sources and allows the dissipated energy to be absorbed directly by the liquid-helium cooling bath rather than by the the magnet. No effort has yet been made to address the effects of interfacial motions in dry-wound solenoids because their quench characteristics are presently dominated by internal, rather than external, disturbances.

\subsection{Summary}

There are two common methods for stabilizing the sliding behavior of a mechanical system. The first is to select frictional surfaces with inherently stable frictionvelocity characteristics. Equation 6.10 indicates that abrupt conductor motions are impossible in frictionally-overdamped $(\delta \mu / \delta \dot{z} \gg 0)$ sliding systems. Positive friction-velocity characteristics are typically associated with the interfacial creep of one member of the sliding pair. No known material demonstrates steady-state creep at liquid-helium temperatures, hence, frictional stabilization via material selection is not a viable method for preventing abrupt conductor-motion disturbances.

The second approach is to increase the sliding system stiffness to a value comparable to the interfacial junction stiffness. Unstable stick-slips can be virtually eliminated if the potential slip distance is reduced to below a typical asperity junction diameter.

Unstable motions in a superconducting magnet can occur on several physical levels. Representative models for three different dimensional scales were developed; these correspond to: the deflection of short (less than one turn) conductor segments; motions involving the displacement of whole conductor turns; and elastic deformation encompassing the entire winding body. Chapter 7 describes an attempt to apply very preliminary versions of these conductor stiffness models to the design of a small superconducting test coil. 


\section{References for Chapter 6}

1. Rabinowicz, E. A study of the stick-slip process Friction and Wear: Proc. Symp. on Friction and Wear, Detroit, 1957 Elsevier, New York (1959) 149-164.

2. Rabinowicz, E. The intrinsic variables affecting the stick-slip process Proc. Phys. Soc. London 71 (1958) 668-675.

3. Courtney-Pratt, J.S., and Eisner, E. The effect of a tangential force on the contact of metallic bodies Proc. Roy. Soc. A 238 (1957) 529-550.

4. Greenwood, J.A., and Tabor, D. The properties of model friction junctions Proc. Conf. on Lubrication and Wear Institution of Mechanical Engineers, London (1957) 314-318.

5. Kuhlmann-Wilsdorf, D. Dislocation concepts in friction and wear Fundamentals of Friction and Wear of Materials American Society for Metals, Metals Park, OH (1981) 119-186.

6. Suh, N.P. Tribophysics Prentice-Hall, Englewood Cliffs, NJ (1986) pp. 103-153.

7. Rabinowicz, E. The nature of the static and kinetic coefficients of friction J. Appl. Phys. 22 (1951) 1373-1379.

8. Bowden, F.P. A review of the friction of solids with particular reference to the conference papers Proc. Conf. on Lubrication and Wear Institution of Mechanical Engineers, London (1957) 239-245.

9. Ogata, K. Modern Control Engineering Prentice-Hall, Englewood Cliffs, NJ (1970).

10. Derjaguin, B.V., Push, V.E., and Tolstoi, D.M. A theory of stick-slip sliding of solids Proc. Conf. on Lubrication and Wear Institution of Mechanical Engineers, London (1957) 257-268.

11. Kensley, R.S., Maeda, H., and Iwasa, Y. Transient slip behaviour of metal/insulator pairs at 4.2 K Cryogenics 21 (1981) 479-489.

12. Kensley, R.S., Maeda, H., and Iwasa, Y. Frictional disturbances in superconducting magnets IEEE Trans. MAG-17 (1981) 1068-1071.

13. Burwell, J.T., and Rabinowicz, E. The nature of the coefficient of friction $J$. Appl. Phys. 24 (1953) 136-139.

14. Grosch, K.A. The relation between the friction and viscoelastic properties of rubber Proc. Roy. Soc. London A 274 (1963) 21-39.

15. Ludema, K.C., and Tabor, D. The friction and visco-elastic properties of polymeric solids Wear 9 (1966) 329-348.

16. Yannas, I.V. Introduction to Polymer Science and Engineering. A set of Lecture 
Notes. MIT, Cambridge (1981).

17. Yannas, I.V., and Luise, R.R. Distinction between two molecular mechanisms for deformation of glassy amorphous polymers J. Macromol. Sci. - Phys. B21 (1981) 443-474.

18. McClintock, F.A., and Argon, A.S. Mechanical Behavior of Materials Addison-Wesley, Reading, MA (1966) pp. 238-243.

19. Williams, M.L., Landel, R.F., and Ferry, J.D. The temperature dependence of relaxation mechanisms in amorphous polymers and other glass-forming liquids $J$. Amer. Chem. Soc. 77 (1955) 3701-3706.

20. Moore, D.F., and Geyer, W. A review of adhesion theories for elastomers Wear 22 (1972) 113-141.

21. Briggs, G.A.D., and Briscoe, B.J. The dissipation of energy in the friction of rubber Wear 35 (1975) 357-364.

22. Chernyak, Y.B., and Leonov, A.I. On the theory of the adhesive friction of elastomers Wear 108 (1986) 105-138.

23. Michael, P.C., Aized, D. Rabinowicz, E., and Iwasa, Y. Mechanical properties and static friction behaviour of epoxy mixes at room temperature and at 77 K Cryogenics 30 (1990) 775-786.

24. Boyer, R.F. Dependence of mechanical properties on molecular motion in polymers Poly. Eng. Sci. 8 (1968) 161-185.

25. Suh, N.P. op. cit. pp. 237-248.

26. Urata, M., and Maeda, H. Stabilization of superconducting dry solenoids IEEE Thans. MAG-25 (1989) 1528-1531.

27. Bowden, F.P., and Rowe, G.W. The friction and the mechanical properties of solid krypton Proc. Roy. Soc. A 228 (1955) 1-9.

28. Coffin, L.F. Some metallurgical aspects of friction and wear Friction and Wear: Proc. Symp. on Friction and Wear, Detroit, 1957 Elsevier, New York (1959) 149164.

29. Bowden, F.P., and Childs, T.H.C. The friction and deformation of clean metals at very low temperatures Proc. Roy. Soc. A 312 (1969) 451-466.

30. Frost, H.J., and Ashby, M.F. Deformation Mechanism Maps. The Plasticity and Creep of Metals and Ceramics Pergamon Press, New York, (1982) pp 1-16.

31. McClintock, F.A., and Argon, A.S. op. cit. pp. 625-636.

32. Suh, N.P. op. cit. pp.35-41.

33. Baber, B.B., Blackstone, W.R., Burton, R.A., Ku, P.M., and Russell, J.A. Lubrication Research and Test Method Development for Aerospace Propulsion 
Systems Technical Document APL-TDR-64-50 Wright-Patterson Air Force Base, OH (1964).

34. Russell, J.A., Campbell, W.E., Burton, R.A., and Ku, P.M. Boundary lubrication behavior of organic films at low temperatures ASLE Thans. 8 (1965) 45-58.

35. Zisman, W.A. Friction, durability and wettability properties of monomolecular films on solids Friction and Wear: Proc. Symp. on Friction and Wear, Detroit, 1957 Elsevier, New York (1959) 149-164.

36. Rabinowicz, E., and Rightmire, B.G. Technical Report on a Project on LowSpeed Sliding Lubrication Laboratory, MIT (1956) Submitted in fulfillment of Office of Naval Research contract number NR 065-335.

37. McCrum, N.G., Read, B.E., and Williams, G. Anelastic and dielectric effects in polymer solids John Wiley, New York (1967) pp. 128-130.

38. McCrum, N.G., Read, B.E., and Williams, G. op. cit. pp. 180-182,371,496.

39. Peterson, M.B., and Ramalingam, S. Coatings for tribological applications Fundamentals of Friction and Wear of Materials American Society for Metals, Metals Park, OH (1981) 119-186.

40. Petty, E.R. A low-temperature inflection in the ternperature-dependence of hardness of pure metals $J$. Inst. Met. 89 (1960) 123-124.

41. Atkins, A.G., Silverio, A., and Tabor, D. Indentation hardness and the creep of solids J. Inst. Met. 94 (1966) 369-378.

42. Atkins, A.G., and Tabor, D. Hardness and deformation properties of solids at very high temperatures Proc. Roy. Soc. A 292 (1966) 441-459.

43. Mulhearn, T.O., and Tabor, D. Creep and hardness of metals: a physical study J. Inst. Met. 89 (1960) 7-12.

44. Rabinowicz, $\mathbf{E}$. Investigation of size effects in sliding by means of statistical techniques Proc. Conf. on Lubrication and Wear Institution of Mechanical Engineers, London (1957) 276-280.

45. Williarns, J.E.C., Neuringer, L.J., Bobrov, E., Weggel, R., Ruben, D.J, and Harrison, W.G. Magnet system of the $500 \mathrm{MHz}$ spectrometer at the Francis Bitter National Magnet Laboratory: I. Design and development of the magnet Rev. Sci. Instrum. 52 (1981) 649-656.

46. Tsukamoto, O., Takao, T., and Honjoh, S. Estimating the size of disturbance due to conductor motion in superconducting windings IEEE Trans. MAG-24 (1988) 1182-1185.

47. Maeda, H. Mechanical disturbance for a cable-in-substructure superconductor Cryogenics 24 (1984) 208-210. 
48. Crandall, S.H., Dahl, N.C., and Lardner, T.J. An Introduction to the Mechanics of Solids McGraw-Hill, New York (1978) pp. 384-385.

49. Williams, J.E.C., and Bobrov, E.S. Magnet system of the $500 \mathrm{MHz} \mathrm{NMR}$ spectrometer at the Francis Bitter National Magnet Laboratory: II. Disturbances, quenches, and training. Rev. Sci. Instrum. 52 (1981) 657-661.

50. Ige, O.O., MacInturff, A.D., and Iwasa, Y. Acoustic emission monitoring results from a fermi dipole Cryogenics 26 (1986) 131-140.

51. Williams, J.E.C., Pourrahimi, S., Iwasa, Y., and Neuringer, L.J. $600 \mathrm{MHz}$ spectrometer magnet IEEE Trans. MAG-25 (1989) 1767-1770. 


\section{Development of a dry-wound insert coil}

The electromagnetic-spring-friction force model suggests two possible approaches for achieving low-temperature sliding stability. The first approaches presupposeu the existence of material pairs whose friction coefficient markedly increases with sliding speed. Because of thermal activation limitations at $4.2 \mathrm{~K}$, the prospect of finding such inherently-stable low-temperature sliding pairs is very unlikely. The second approach relies on a high conductor stiffness to limit potential microslips to displacements smaller than the average junction diameter.

Section 6.4 suggests one method for markedly increase the effective axial stiffness of a collection of conductor segments; that is, by eliminating interconductor gaps and transforming conductor segments into a single structural unit. In impregnated magnets the axial gaps are eliminated using a filler material such as epoxy resin. As discussed previously, the use of epoxy fillers is undesirable from a manufacturing viewpoint because of the increased number of processing steps required. For drywound magnets, a second possibility is to vary the conductors' axial stiffnesses during energization, that is, to allow the conductors to move together freely during low-field operation where their stability margins are greatest. As the conductors move the winding's interconductor gaps are eliminated; this increases their effective axial stiffnesses to values comparable with those in an impregnated winding.

The chapter is organized as a case study involving the design, construction, and operation of a small, high-field NbTi solenoid. The magnet's design is based on the premise that conductor motions in dry-wound solenoids are permissible so long as the energy dissipated during these motions remains below the magnet's stability energy margin. The test program's experimental results suggest that this approach was at least partially successful.

\subsection{Structural characterization of dry-wound solenoids}

Dry-wound magnets offer a lower-cost but presently unreliable alternative for the construction of adiabatic high-field solenoids. Dry-wound construction is frequent' $y$ 
employed in niobium-titanium-based ( $\mathrm{NbTi}$ ) superconducting solenoids that produce central inductions up to approximately $7 \mathrm{~T}$. Frictional heating disturbances are cited as the principal limitation to reliable operation. Operating conditions above $7 \mathrm{~T}$ allegedly favor the use of epoxy-impregnated windings; epoxy impregnation prevents wire movements by transforming the entire winding into a single monolithic structure.

Very little information is available in the literature to account for this supposed $7 \mathrm{~T}$ operating limit. The most comprehensive structural investigation of dry-wound solenoids was reported by Urata and Maeda concerning their efforts to develop a prototype $7.2 \mathrm{~T}$ magnet; ${ }^{[1,2]}$ partial results from this study were presented in §5.3.2. Their frictional results confirm that Formvar-insulated conductors demonstrate marginal sliding stability.

A common approach aimed at improving dry-wound coil performance is to use a very large conductor tension during manufacture so that the resulting radial preload suppresses the conductor's tendency towards microslip behavior. Urata and Maeda devised such a coil and still observed microslip-induced premature quenches at $\sim 85 \%$ of their coil's critical current. Tightly-wound coils generally do not perform as well as anticipated because the conductor's dimensional tolerance is typically much larger than its radial elastic deformation; this produces conductor segments that are less well supported than predicted and hence prone to slip. The efficacy of increasing the winding tension still further is limited both by the conductor's tensile yield strength and by the tendency towards circumferential buckling in the coil form and in the coil's inner layers. ${ }^{[3]}$

Urata and Maeda also investigated more moderately tensioned coils; these coils demonstrated considerably larger slip distances and markedly lower initial quench currents. However, the moderately tensioned coils trained progressively, eventually reaching their short-sample critical currents after about ten charging cycles. After determining that this training cycle was too long for their intended application, they ultimately settled on a more labor intensive, epoxy-impregnated version of the winding which achieved its full short sample current during its second training cycle. ${ }^{[2]}$ 
By relying on the rapid attainment of the magnet's short sample critical current as an objective performance criterion, Urata and Maeda applied a much more extreme condition than required for most practical applications. Very few superconducting magnets are designed to operate exactly at their critical current because any perturbation could lead to quenching. Most magnets are operated slightly below their critical currents; this provides a stability margin against the potential disturbances sources listed in $§ 3.1$. In other applications, such as nuclear magnetic resonance (NMR) spectrometers the magnet's operating current is reduced still further, perhaps to $\sim 75 \%$ of the critical current; this improves the magnet's temporal stability which deteriorate rapidly above $\sim 75 \% I_{c}$ due to flux-creep induced dissipation.

\subsection{Extension of dry-winding technique to high-field solenoids}

Urata and Maeda's investigations show that the quench and training behaviors of simple solenoids can be modified by varying the conductor tension during manufacture. Premature quenches may be caused either by small axial motions at high stress levels, or by large motions at more moderate stresses. Additionally, $\$ 5.3$ reports that disturbances in tightly wound coils tend to recur during subsequent charging cycles; these recurrent motions are attributed to the temporary elastic displacement of small conductor segments in an otherwise rigid coil. In more loosely wound coils the potential for conductor motion is gradually exhausted as the conductor segments shift to more stable equilibrium positions.

Several factors influence the design of high-field solenoids. One is that the conductor's critical current density decreases markedly with increasing magnetic induction. Figure 2.4 shows an example of this effect for a hypothetical NbTi superconductor. The continued reduction in NbTi's current density above $6 \sim 7 \mathrm{~T}$ eventually makes radial subdivision of a solenoid into separate, nested coils and increasingly necessary construction alternative. The total quantity of superconductor required can be markedly reduced by grading the conductor's current density to match the local magnetic induction.

A second issue confronting a magnet designer is that the magnet's stability energy margin likewise decreases with increasing induction. The magnitude of this 
effect is demonstrated in Figure 3.1; the conductors in high field regions of a coil are considerable less tolerant of abrupt mechanical disturbances than are the low field conductors.

An attendant concern in the development of a radially-subdivided solenoid is the potential for radial separation between adjacent conductor layers. Middleton and Trowbridge analyzed this tendency in isotropic, radially-continuous, infinitelength solenoids. ${ }^{4]}$ Figure 7.1 presents a graphical representation of their results. They concluded that the radial stress state in a solenoid is determined principally by its normalized radial thickness, $\alpha$, and the ratio of the magnetic inductions at the winding's inner and outer radii, $B_{a 2} / B_{a 1}$. Figure 7.1 depicts the presence of tensile and compressive radial stresses within a winding. For a dry-winding, the appearance of tensile stresses in Figure 7.1 is equivalent to the onset of radial separation; contact can be maintained only in the presence of compressive radial stresses.

Although radial separation between a winding and its coil form is acceptable, perhaps even desirable in some instances, ${ }^{[5-7]}$ the occurrence of interlayer separation constitutes a gross mechanical instability that should be scrupulously avoided. There are several methods for reducing a coil's tendency towards radial separation. The first is to select the coil's radial build and field increment so that the distributed electromagnetic forces naturally enforce radial continuity. ${ }^{[4]}$ The second is to use sufficient winding tension during manufacture to circumvent this radial separation tendency. ${ }^{[3,5]}$ A third approach is to externally reinforce the winding; contact is maintained by limiting the outward expansion of the winding's outer layers to a value less than the unsupported expansion of its inner layers.

\subsection{Overview of insert coil design}

The case study pursued during this investigation involves the development of the inner, or insert, coil in a two coil solenoidal magnet. The coil's specifications require it to be dry-wound using a rectangularly cross-sectioned Formvar-insulated $\mathrm{NbTi}$ superconductor. The coil pair should produce a maximun, combined central induction of $8.5 \mathrm{~T}$ and demonstrate minimal training. This central induction is midway between the alleged 7-T upper limit for dry-winding viability and NbTi's 


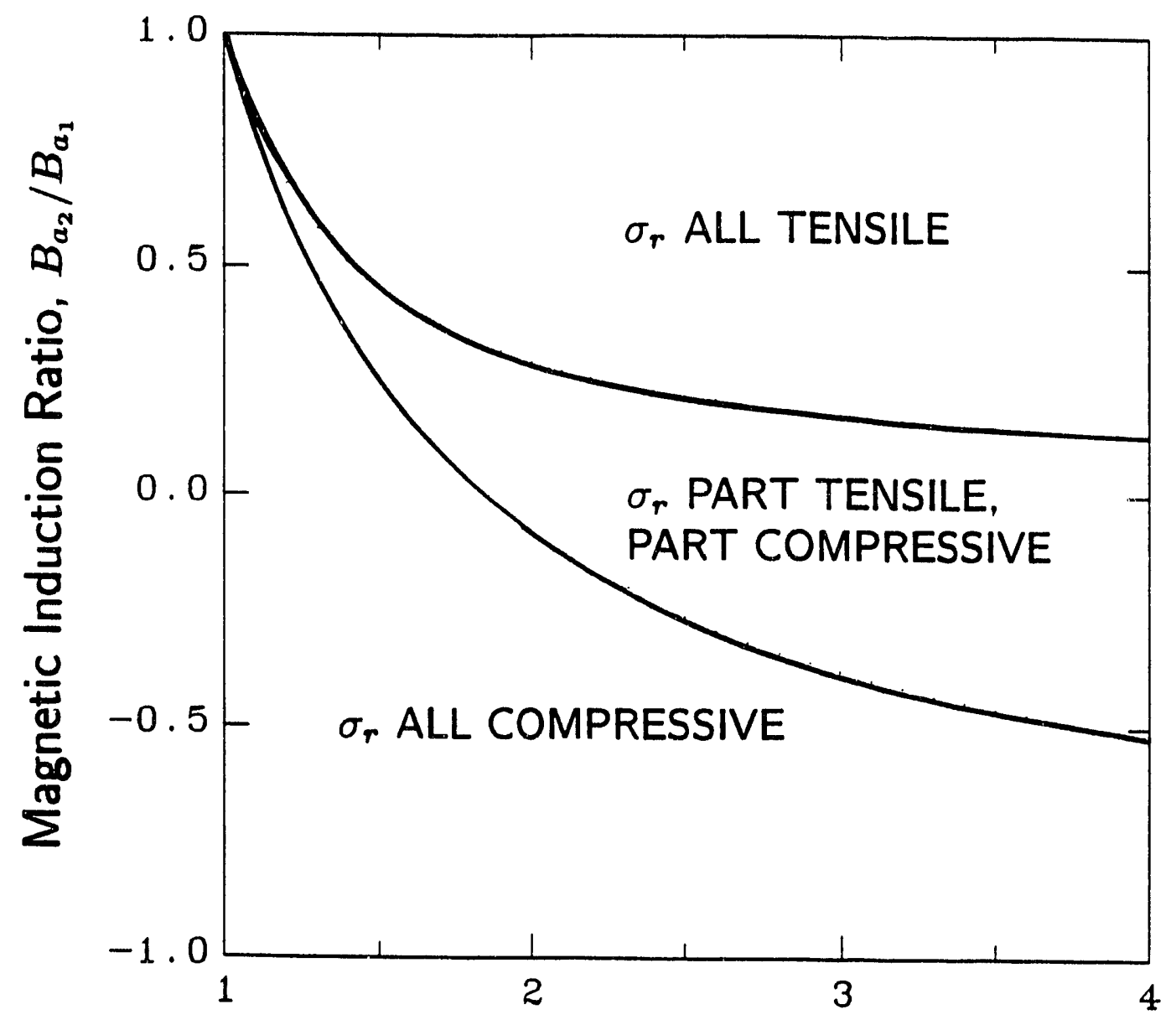

Normalized Outer Radius, $\alpha=a_{2} / a_{1}$

Figure 7.1: Occurrence of tensile and compressive radial stresses in an isotropic. radially-connected, infinite-length solenoid. The radial stress state is determined by the winding's normalized radial thickness $\alpha$ and the ratio of internal/external magnetic induction, $B_{a_{2}} / B_{a_{1}} .4$ ? The appearance of tensile radial stresses is equivalent to the onset of radial separation in a dry winding. 
maximum $4.2-\mathrm{K}$ critical flux density of $\sim 10 \mathrm{~T}$. The remainder of the coil's parameters were selected based on their compatability with laboratory facilities existing at the Francis Bitter National Magnet Laboratory (FBNML) where the coil was tested.

\subsubsection{Electromagnetic design}

The insert coil was designed to operate in conjunction with the 6B-Bitter solenoid facility at FBNML. Bitter solenoids constitutes a class of compact, watercooled, high-field/high-current-density, resistive electromagnets. ${ }^{[8]}$ The $6 \mathrm{~B}$ magnet has a $147 \mathrm{~mm}$ ( $5.79 \mathrm{in}$ ) diameter clear bore and produces a $6.5 \mathrm{~T}$ central induction at a 20,000 A operating current. This 20,000 A current represents the combined output from two of the labs four $2.5 \mathrm{MW}$ d.c. generators.

The conductor used for the superconducting insert was selected from stock remaining from a previous magnet development project; it was donated to this thesis by Kobe Steel. The conductor's specifications are listed in Table 7.1. The conductor was chosen for its relatively high superconducting volume fraction and small crosssectional area. Because a rectangular cross-sectioned wire is used the winding's superconductor volume fraction is essentially the same as that in the conductor. A high superconducting volume fraction allows a coil with a small radial build to produce a marked field increment. The conductor's small cross-sectional area limits the coil's operating current to $400 \mathrm{~A}$, a value compatible with stable power supplies available in the lab.

The test coil's inner diameter was selected to appro.imate the dimension of typical high-field magnets and to allow training effects to occur if they are present. Previous conductor motion studies indicate that conductor-motion-induced quenches are only observed in solenoids whose inner diameters exceed a certain minimum value. The Rutherford Laboratory studies ${ }^{[\theta]}$ report that $20 \mathrm{~mm}$ I.D. windings are impervious to conductor-motion-induced degradation whereas $90 \mathrm{~mm}$ I.D. windings train readily. The bore sizes employed during subsequent studies ranged from $50^{[10]}$ $\sim 90 \mathrm{~mm} .{ }^{[11]}$ These dimensions are typical of laboratory research magnets and NMR spectroscopy magnets.

The test coil's outer diameter was limited by the bore of the Bitter solenoid 
Table 7.1: Insert coil conductor specifications.

\begin{tabular}{|c|c|}
\hline Uninsulated conductor width & $1.215(\mathrm{~mm})$ \\
\hline Uninsulated conductor thickness & $0.859(\mathrm{~mm})$ \\
\hline Insulated conductor width, $w$ & $1.262(\mathrm{~mm})$ \\
\hline Insulated conductor thickness, $t$ & $0.919(\mathrm{~mm})$ \\
\hline Copper:superconductor ratio & 0.98 \\
\hline Copper area & $0.517\left(\mathrm{~mm}^{2}\right)$ \\
\hline Non-copper area & $0.527\left(\mathrm{~mm}^{2}\right)$ \\
\hline Estimated total area & $1.160\left(\mathrm{~mm}^{2}\right)$ \\
\hline Superconductor volume fraction & 0.454 \\
\hline
\end{tabular}

Table 7.2: Insert coil specifications.

\begin{tabular}{|c|c|}
\hline Inner dianieter, $2 \mathrm{a}_{1}$ & $76.2(\mathrm{~mm})$ \\
\hline Outer diameter, $2 \mathrm{a}_{2}$ & $87.23(\mathrm{~mm})$ \\
\hline Normalized outer diameter, $\alpha$ & 1.14 \\
\hline Coil length, $2 \mathrm{~b}$ & $126.20(\mathrm{~mm})$ \\
\hline Normalized half-length, $\beta$ & 1.6 \\
\hline Bore tube thickness & $2.67(\mathrm{~mm})$ \\
\hline External reinforcement thickness & $1.02(\mathrm{~mm})$ \\
\hline Layers & 6. \\
\hline Turns/layer & 100. \\
\hline Total turns & 600. \\
\hline Conductor length & 154. \\
\hline Operating current & 400. \\
\hline (m) \\
\hline Self central induction, $B_{0}$ & $2.0(\mathrm{~T})$ \\
\hline Self peak induction, $B_{w}$ & $2.1(\mathrm{~T})$ \\
\hline Combined central induction, $B_{0}^{*}$ & $8.5(\mathrm{~T})$ \\
\hline Combined peak induction, $B_{w}^{*}$ & $8.7(\mathrm{~T})$ \\
\hline
\end{tabular}

"When operated in conjunction with $6 \mathrm{~B}$ Bitter solenoid 
and by the need to provide instrumentation access to the coil's outer surface. The coil length was selected to give good internal field uniformity, consistent with field constraints imposed by the Bitter solenoid. The trade-off between coil length, field uniformity, and superconductor utilization was discussed in $§ 2.4$.

Table 7.2 presents the insert coil specifications. The insert coil dimensions are depicted schematically in Figure 7.2. The $76.2 \mathrm{~mm}$ I.D. insert coil consists of six-layer of rectangularly cross-sectioned wire, wound on a $2.67 \mathrm{~mm}$ thick AISI 304 stainless steel coil form. The magnet is externally reinforced with four layers of $2.5 \mathrm{~mm}$ wide, $0.25 \mathrm{~mm}$ thick beryllium-copper strip to prevent interlayer radial separation during energization.

The coil produces a self, central induction of $2.0 \mathrm{~T}$ at a $400 \mathrm{~A}$ operating current. Figure 7.3 shows the insert coil's peak field load line, BP, when operating in a $6.5-\mathrm{T}$ background central induction provided by the $6 \mathrm{~B}$ Bitter solenoid. The diagonal line running from upper left to lower right in this figure represents the conductor's 4.2$\mathrm{K}$ critical properties. The insert coil's critical point, $P$, represents the coil's $400 \mathrm{~A}$ operating current and 8.7-T combined peak induction. Field mappings of the axial and radial magnetic inductions for both the insert coil and the $6 \mathrm{~B}$ Bitter solenoid are included in Appendix A.

\subsubsection{Mechanical design}

The insert coil's mechanical design was pursued with regard to the conductor motion model presented in $\$ 5.2$. The radial component of the magnetic induction near the solenoid's ends produces axially directed electromagnetic forces on the conductors. Individual conductor motions require: i) axial gaps between adjacent conductors and ii) that the applied force on a conductor segment exceeds its static friction force. Axial gaps are created by variations in conductor placement during manufacture and by axial thermal contractions during cooldown; these axial interconductor gaps are virtually unavoidable in practice.

The insert coil was constructed using minimal conductor tension. The intent of this construction is to encourage incipient conductor motions to occur far below the winding's critical point where its stability margin is greatest. This approach was inspired by Urata and Maeda's single coil test data which show that conductor motions 


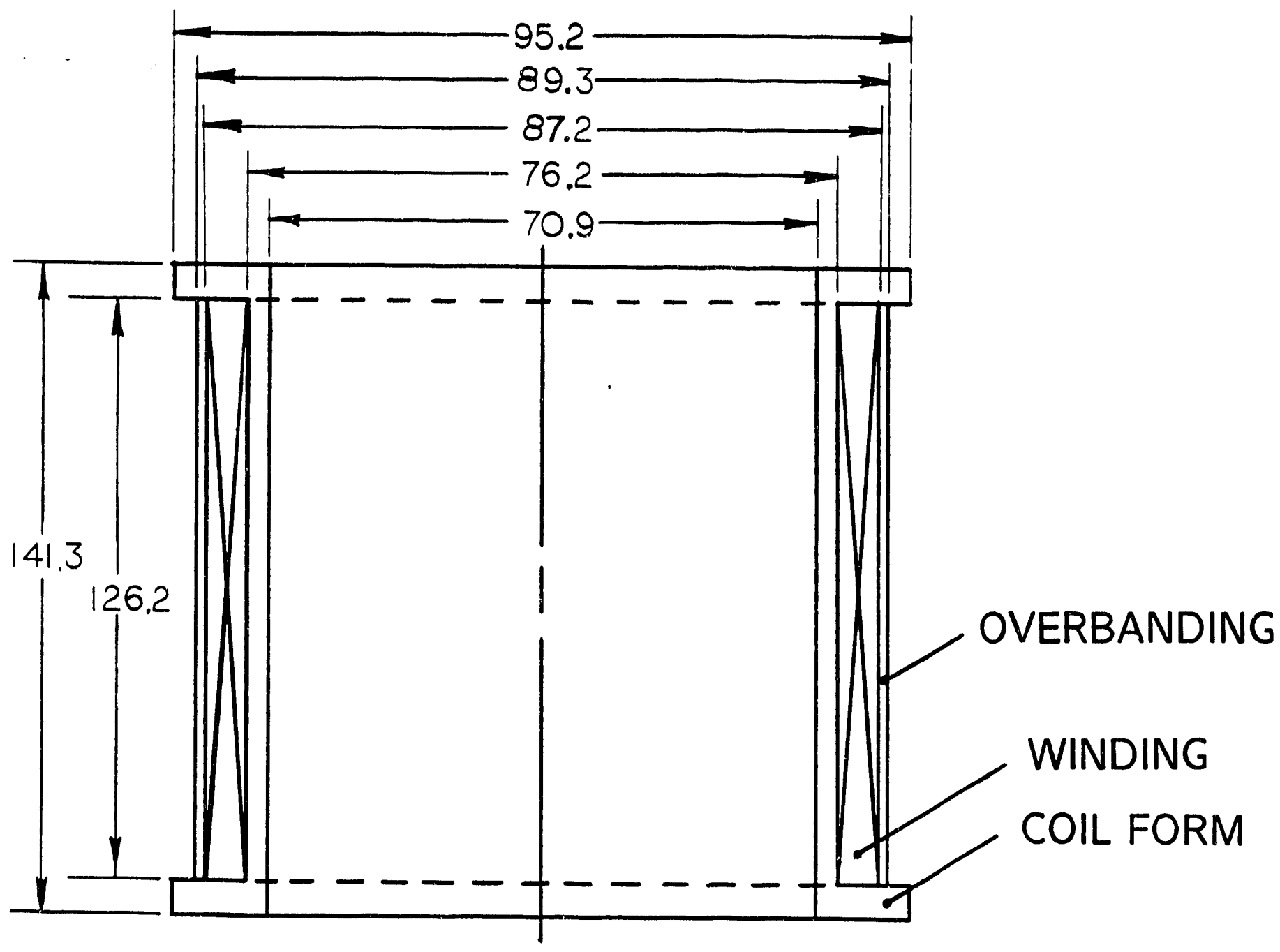

Figure 7.2 : Insert coil dimensions (in $\mathrm{mm}$ ). 


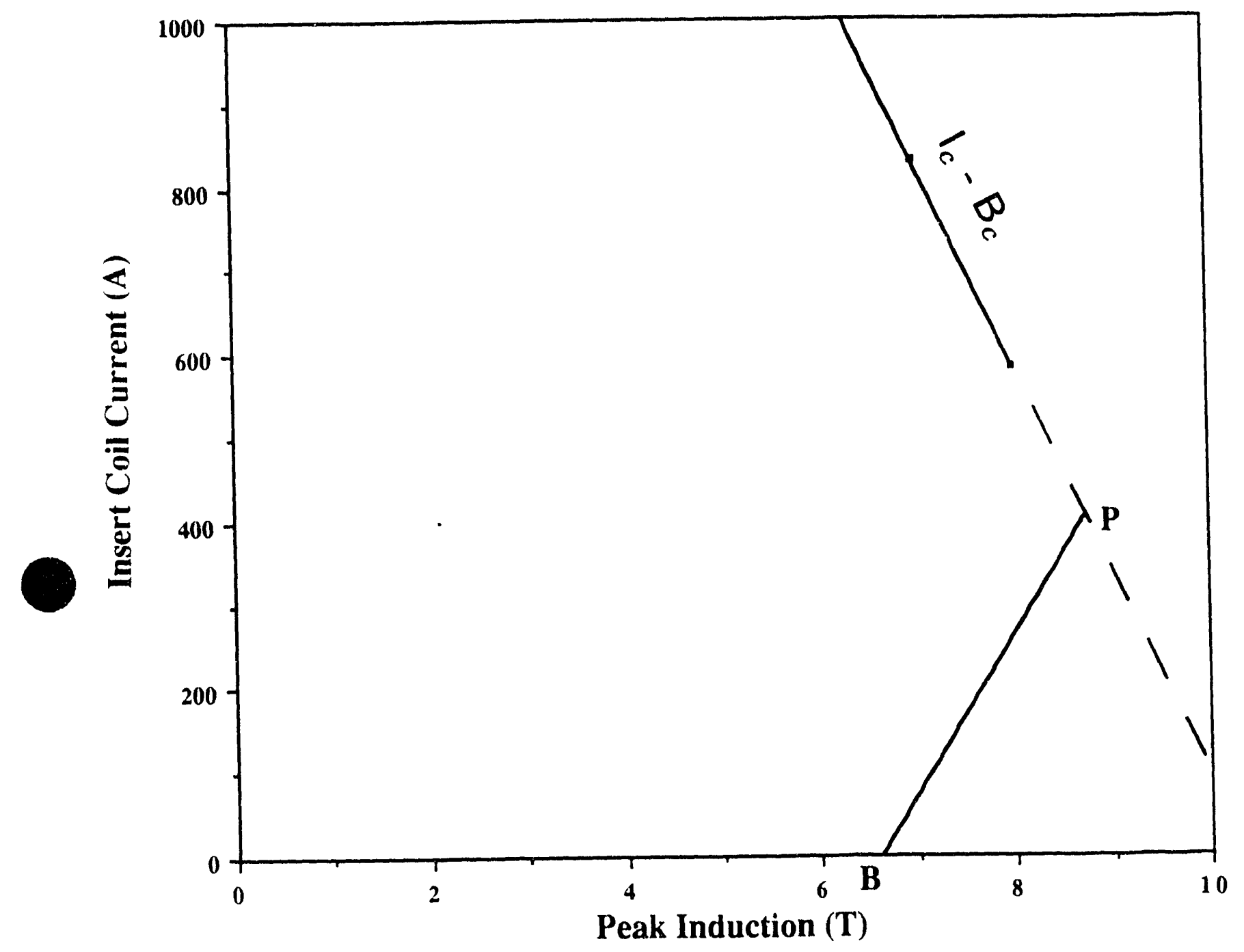

Figure 7.3: Insert coil load line in $6.5-\mathrm{T}$ background central induction. 
in loosely wound coils eventually cease as preexisting gaps between adjacent conductors are eliminated by the winding's axial electromagnetic compression. ${ }^{[2]}$ Thus, the insert coil's design attempts to reduce conductor-motion-induced quenches by eliminating as many of the winding's axial gaps as early in the charging sequence as possible.

The coil's mechanical stress state was analyzed primarily following the methodology put forth by Bobrov and Williams. ${ }^{[\theta]}$ Their analysis was supplemented using contributions from Arp. ${ }^{[5]}$ The stress analysis was implemented numerically using dedicated programs developed on the VAX computer system at MIT's Plasma Fusion Center. The insert coil is modeled as an inhomogeneous system consisting of eleven concentric cylindrical shells, or coil elements. The constitutive relations for each shells are given by:

$$
\begin{aligned}
& \epsilon_{r}=\frac{\sigma_{r}}{E_{r}}-\nu_{r \theta} \frac{\sigma_{\theta}}{E_{\theta}}-\nu_{r z} \frac{\sigma_{z}}{E_{z}} \\
& \epsilon_{\theta}=-\nu_{\theta r} \frac{\sigma_{r}}{E_{r}}+\frac{\sigma_{\theta}}{E_{\theta}}-\nu_{\theta z} \frac{\sigma_{z}}{E_{z}} \\
& \epsilon_{z}=-\nu_{z r} \frac{\sigma_{r}}{E_{r}}-\nu_{z \theta} \frac{\sigma_{\theta}}{E_{\theta}}+\frac{\sigma_{z}}{E_{z}} .
\end{aligned}
$$

The following relations between the orthotropic elastic constants apply:

$$
\frac{\nu_{r \theta}}{E_{\theta}}=\frac{\nu_{\theta r}}{E_{r}} ; \quad \frac{\nu_{\theta z}}{E_{z}}=\frac{\nu_{z \theta}}{E_{\theta}} ; \quad \frac{\nu_{z r}}{E_{r}}=\frac{\nu_{r z}}{E_{z}} .
$$

Particular features of the analysis include the superposition of stresses arising from the conductor tension during winding, differential contractions during cooldown, and distributed electromagnetic body forces during energization. The analysis also incorporates constraints to accommodate radial separation within the winding or between the winding and its coil form.

Table 7.3 presents the material properties for each of the model's eleven cylindrical coil elements. The values were obtained from the published literature. ${ }^{[1,7,12-14]}$ The derivations of winding's equivalent orthotropic elastic modulii, poisson's ratios, and directional thermal contractions are presented in Appendix B. Although both the coil form and overbanding are actually isotropic materials, this presents no major difficulties in the analysis; the anisotropic solution for a cylindrical shell 
Table 7.3: Insert-coil model's materials properties.

\begin{tabular}{|c|c|c|c|}
\hline Coil element & Coil Form & Winding & Overbanding \\
\hline Element \# & 0 & $1-6$ & $7-10$ \\
\hline Material & $\begin{array}{l}\text { Stainless } \\
\text { Steel }\end{array}$ & $\begin{array}{l}\text { NbTi-based } \\
\text { Superconductor }\end{array}$ & $\begin{array}{l}\text { Beryllium- } \\
\text { Copper }\end{array}$ \\
\hline $\begin{array}{r}293 \mathrm{~K} \text { Radial } \\
\text { Modulus (GPa) }\end{array}$ & 185 & 45.9 & 121 \\
\hline $\begin{array}{r}293 \mathrm{~K} \text { Hoop } \\
\text { Modulus (GPa) }\end{array}$ & 185 & 85.8 & 121 \\
\hline$\gamma$ at $293 \mathrm{~K}$ & 1 & 1.37 & 1 \\
\hline$\nu_{r \theta}$ at $293 \mathrm{~K}$ & 0.33 & 0.33 & 0.33 \\
\hline$\nu_{\theta r}$ at $293 \mathrm{~K}$ & 0.33 & 0.18 & 0.33 \\
\hline $\begin{array}{r}4.2 \mathrm{~K} \text { Radial } \\
\text { Modulus }(\mathrm{GPa})\end{array}$ & 205 & 63.0 & 134 \\
\hline $\begin{array}{r}4.2 \mathrm{~K} \text { Hoop } \\
\text { Modulus }(\mathrm{GPa})\end{array}$ & 205 & 89.3 & 134 \\
\hline$\gamma$ at $4.2 \mathrm{~K}$ & 1 & $1 . \overline{19}$ & 1 \\
\hline$\nu_{r \theta}$ at $4.2 \mathrm{~K}$ & 0.33 & 0.33 & 0.33 \\
\hline$\nu_{\theta r}$ at $4.2 \mathrm{~K}$ & 0.33 & $\overline{0.23}$ & 0.33 \\
\hline $\begin{array}{r}\text { Radial Thermal } \\
\text { Contraction } \\
\text { Strain, } \Delta_{r}^{*}\end{array}$ & -0.00306 & -0.00377 & -0.00317 \\
\hline $\begin{array}{r}\text { Hoop Thermal } \\
\text { Contraction } \\
\text { Strain, } \Delta_{\theta}^{*}\end{array}$ & -0.00306 & -0.00279 & -0.00317 \\
\hline
\end{tabular}

* Cumulative between 293 and $4.2 \mathrm{~K}$. 
converges toward the isotropic solution as $\gamma \rightarrow 1 . \gamma$ is a measure of the shell's mechanical anisotropy; $\gamma=\sqrt{E_{\theta} / E_{r}}$.

\subsubsection{In-plane stresses}

The axial stress in a dry-wound solenoid is generally much smaller than and uncoupled from the in-plane stresses; hence it is usually dropped from the constitutive relations, Eq. 1, resulting in the following two dimensional problem.

$$
\begin{aligned}
\epsilon_{r} & =\frac{\sigma_{r}}{E_{r}}-\nu_{r \theta} \frac{\sigma_{\theta}}{E_{\theta}} \\
\epsilon_{\theta} & =-\nu_{\theta r} \frac{\sigma_{r}}{E_{r}}+\frac{\sigma_{\theta}}{E_{\theta}} \\
\sigma_{r} & =\frac{E_{r}}{1-\nu_{r \theta} \nu_{\theta r}}\left[\epsilon_{r}+\nu_{r \theta} \epsilon_{\theta}\right] \\
\sigma_{\theta} & =\frac{E_{\theta}}{1-\nu_{r \theta} \nu_{\theta r}}\left[\epsilon_{\theta}+\nu_{\theta r} \epsilon_{r}\right]
\end{aligned}
$$

For small strains, the in-plane strains are defined by geometric compatability

$$
\begin{aligned}
& \epsilon_{r}=\frac{d u(r)}{d r} \\
& \epsilon_{\theta}=\frac{u(r)}{r,}
\end{aligned}
$$

and the in-plane stresses by static equilibrium

$$
r \frac{d \sigma_{r}}{d r}+\left(\sigma_{r}-\sigma_{\theta}\right)=-r f(r)
$$

The $f(r)$ term in Eq. 7.4 represents any distributed body force acting on the cylinder.

Multiple substitutions of Eq. 7.3 into 7.2 and Eq. 7.2 into 7.4 yield the following relation between the radial displacement, $u(r)$, and the applied body forces.

$$
r \frac{d^{2} u}{d r^{2}}+\frac{d u}{d r}-\left[\frac{E_{\theta}}{E_{r}}\right] \frac{u}{r}=-\frac{r f(r)\left(1-\nu_{r \theta} \nu_{\theta r}\right)}{E_{r}}
$$

The homogeneous solution for $\check{\mathrm{q}} \mathrm{q} .7 .5$, with $\gamma=\sqrt{E_{\theta} / E_{r}}$, is given by:

$$
u_{h}(r)=A_{1} r^{\gamma}+B_{1} r^{-\gamma}
$$


The particular solution is determined by the specific forcing function $f(r)$. The corresponding strains and stresses are found by substituting these radial displacements into Eqs. 7.3 and 7.2 and applying the appropriate boundary conditions.

There are several possible solutions to the insert coil's analytical model depending on the boundary conditions imposed between the adjacent coil elements. The most general solution is achieved by requiring continuous radial stresses and continuous radial displacements at each interface; Eq. 7.7 presents a mathematical description of these boundary conditions.

$$
\begin{gathered}
\sigma_{r_{i}}\left(r_{i+1}\right)=\sigma_{r_{i+1}}\left(r_{i+1}\right) \\
u_{i}\left(r_{i+1}\right)=u_{i+1}\left(r_{i+1}\right) .
\end{gathered}
$$

The subscript $i$ denotes the coil element's radial position which is numbered outward from the coil form. The ith element's unstressed inner radius is similarly located at $r_{i}$.

Radial contact between coil elements can be maintained only in the presence of compressive radial stresses. In the event of a tensile radial stress solution, the boundary conditions may be readjusted to accommodate radial separation between coil elements by relaxing the condition on continuous radial displacements and imposing zero radial pressure on both sided of the interface.

$$
\begin{aligned}
\sigma_{r_{i}}\left(r_{i+1}\right) & =0 \\
\sigma_{r_{i+1}}\left(r_{i+1}\right) & =0
\end{aligned}
$$

In all cases the radial pressures are identically zero along both of the coil's free surfaces, namely, at the inner radius of the coil form and the overbanding's outer radius.

$$
\begin{aligned}
\sigma_{r_{0}}\left(r_{0}\right) & =0 \\
\sigma_{r_{10}}\left(r_{11}\right) & =0
\end{aligned}
$$

The cumulative stress state that arises during magnet operation is the result of residual winding tension stresses, thermal contraction stresses, and reaction stresses to the winding's distributed electromagnetic body forces. One goal of the insert 
coil's mechanical design is to configure the winding tension stresses during manufacture to offset the subsequent thermal contraction stresses to produce a virtually stress-free winding at the start of energization. Because the thermal contraction stresses are determined solely by the coil composition the thermal contraction stress analysis will be presented first, followed by the winding-tension stress analysis. Each analysis uses a small-strain approximation, that is, the radial positions of the coil elements are not updated between stress component calculations. All analyses are performed using the elements' undeformed geometries. The resulting solutions are then superposed to determine the coil's cumulative elastic response.

\subsubsection{Thermal contraction stresses}

One method for deriving the thermal contraction induced stresses is to directly incorporate the coil elements' 293 -to-4.2 $\mathrm{K}$ thermal contractions into Eq. 7.2.

$$
\begin{aligned}
& \epsilon_{r}=\frac{\sigma_{r}}{E_{r}}-\nu_{r \theta} \frac{\sigma_{\theta}}{E_{\theta}}+\Delta_{r} \\
& \epsilon_{\theta}=-\nu_{\theta r} \frac{\sigma_{r}}{E_{r}}+\frac{\sigma_{\theta}}{E_{\theta}}+\Delta_{\theta} .
\end{aligned}
$$

After applying static equilibrium (Eq. 7.4) to the revised constitutive equations (Eq. 7.10) Eq. 7.5 becomes:

$$
r \frac{d^{2} u}{d r^{2}}+\frac{d u}{d r}-\left[\frac{E_{\theta}}{E_{r}}\right] \frac{u}{r}=\left(1-\gamma^{2} \nu_{\theta r}\right) \Delta_{r}+\left(\nu_{r \theta}-\gamma^{2}\right) \Delta_{\theta}
$$

For an anisotropic cylinder the particular solution for the element's thermalcontraction-induced radial displacement is given by:

$$
u_{p}(r)=\frac{\left(1-\gamma^{2} \nu_{\theta r}\right) \Delta_{r}+\left(\nu_{r \theta}-\gamma^{2}\right) \Delta_{\theta}}{\left(1-\gamma^{2}\right)} r
$$

As $\gamma \rightarrow 1 \mathrm{Eq} .7 .12$ becomes singular, hence an alternate solution is needed to satisfy the radial displacement for an isotropic cylinder. The particular solution:

$$
u_{p}(r)=\Delta r
$$

is computible with the thermal strains induced by the stress-free contraction of the isotropic winding elements. 
Figure 7.4 plots the insert coil model's anticipated thermal-contraction-induced radial stress $v s$ radial location. The large tensile radial stresses in the magnet's inner layers are caused principally by the conductor's large radial thermal contraction, $\Delta_{r}$, relative to the rest of the coil. Despite the appearance of these tensile radial stresses, a radially continuous boundary condition is maintained at this stage of the analysis to facilitate the subsequent conductor tension calculations.

\subsubsection{Residual winding stresses}

As a solenoid is wound the tension in each subsequent layer $k, T_{k}$, radially compresses the coil's inner elements, $i=0, \ldots, k-1$. The radial pressure along the outer surface of the $(k-1)$ st layer produced by $T_{k}$ is:

$$
\sigma_{r_{h-1}}\left(r_{k}\right)=\frac{T_{k}}{A} \frac{\left(r_{k+1}-r_{k}\right)}{r_{k}}
$$

$A$ is the conductor's cross-sectional area. The boundary conditions for the rest of the inner coil elements are satisfied using Eqs. 7.7 and 7.9a. The stress state in the coil's outer elements, $i>k$, is unaffected by $T_{k}$. For an $n$-layer coil the net elastic solution at the end of the winding process is given by:

$$
\begin{aligned}
\sigma_{r_{i}}(r) & =\Sigma_{k=1}^{n} \sigma_{r_{i k}}(r) T_{k} & & (i=0, \ldots, n) \\
\sigma_{t_{i}}(r) & =\Sigma_{k=1}^{n} \sigma_{t_{i k}}(r) T_{k} & & (i=0, \ldots, n) \\
u_{i}(r) & =\Sigma_{k=1}^{n} u_{i k}(r) T_{k} & & (i=0, \ldots, n) .
\end{aligned}
$$

$\sigma_{r_{i h}}(r)$ is the radial stress, $\sigma_{t_{i k}}(r)$ the hoop stress, and $u_{i k}(r)$ the radial displacement produced in layer $i$ by the winding tension applied to layer $k$.

This linear, small-strain, elastic coil analysis can be generalized to any arbitrary collection of winding tensions by first solving the analysis using a unit tension winding vector, $T_{k}=1 \quad(k=1, \ldots, n)$. The $\sigma_{r_{i h}}, \sigma_{t_{i k}}$, and $u_{i k}$ derived during the unit tension analysis constitute stress influence matrices. The stress state for any collection of winding tensions can then be determined simply by multiplying these stress influence matrices by the corresponding winding tension vector. Bobrov and Williams ${ }^{[3]}$ have further extended this approach and present a linear programming approach for optimization the winding tension subject to objective constraints imposed at various locations throughout the coil. 


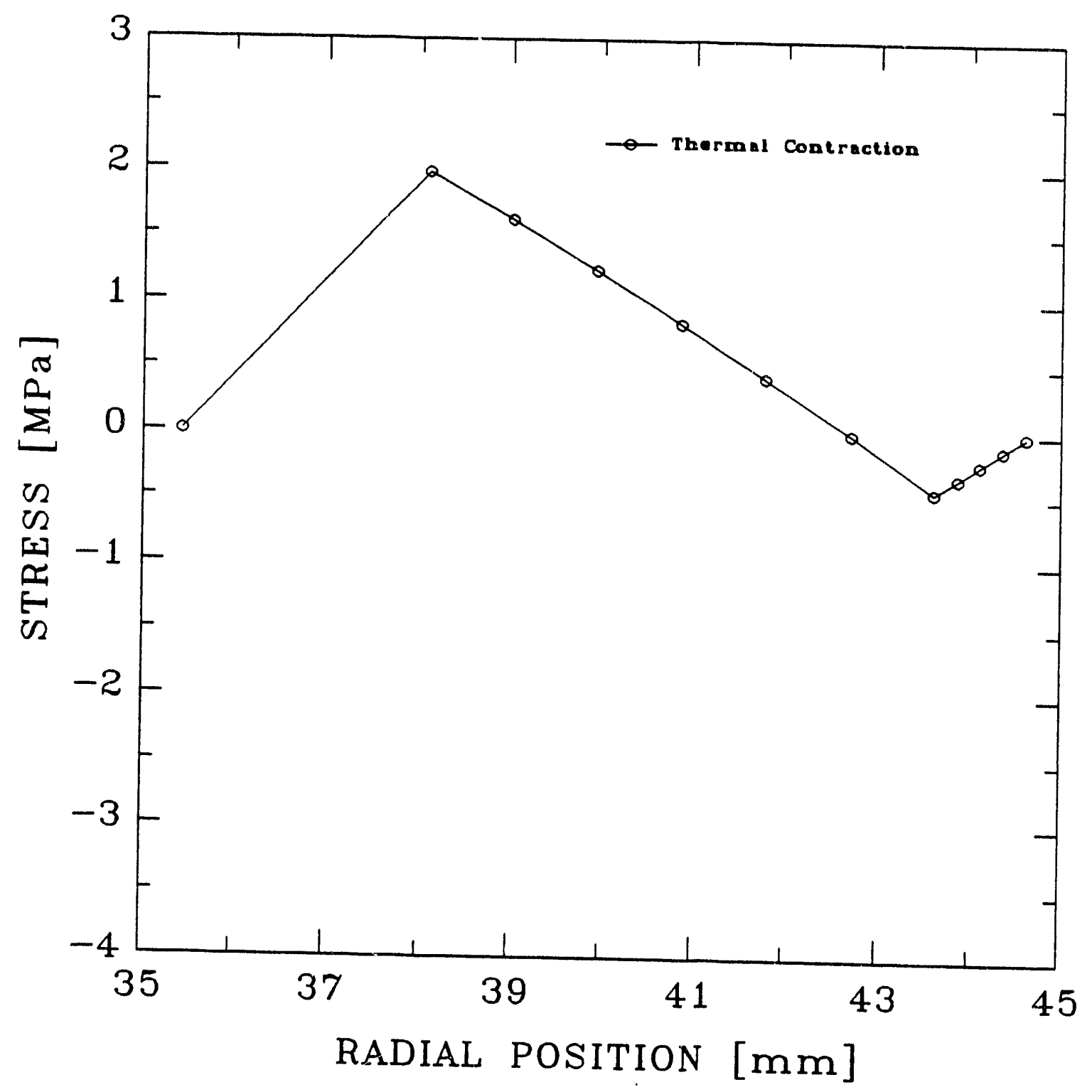

Figure 7.4: Thermal contraction induced radial stresses $v s$ radial position. The open circles along the trace indicate the coil element boundaries. 
The winding tension vector implemented in insert coil design uses tensions of $24.5 \mathrm{~N}$ for the first five conductor layers, $11.5 \mathrm{~N}$ in the 6th conductor layer, and $5 \mathrm{~N}$ in the four overbanding layers. Figure 7.5 shows the residual winding-tensioninduced radial stress $v s$ radial position corresponding to this winding tension vector. Traces included in Figure 7.5 also depict the thermal-contraction-induced radial stresses and the cumulative stress state following both winding and cool-down. The winding tension vector wes selected to produce a slightly compressive cumulative radial stress following cool-down for two reasons. One is that the selected winding tension vector lies at the lower range of our winding apparatus capabilities where tension regulation is not likely to be highly accurate. The second is that the coil's assumed mechanical properties have not been verified experimentally.

\subsubsection{Electromagnetic stresses}

The electromagnetic body force in a solenoidal winding is given by:

$$
f(r)=j_{a v e} B_{z}(r)
$$

where $j_{a v e}$ is the average current density in the winding, and $B_{z}(r)$ is the axial component of the magnetic induction at the radial location $r$. For a uniform current density coil, the axial magnetic induction varies approximately linearly across the winding.

$$
B_{z}(r)=B_{c}-d B r
$$

where $d B$ is the induction gradient across the coil's build and $B_{c}$ is the extrapolated value of the induction at the coil axis. Substituting Eq. 7.17 into 7.16 and Eq. 7.16 into 7.5 yields the particular solution for the winding's electromagnetic-forceinduced radial displacement:

$$
u_{p}(r)=-\frac{j_{a v e} B_{c}\left(1-\nu_{r \theta} \nu_{\theta r}\right)}{\left(4-\gamma^{2}\right) E_{r}} r^{2}+\frac{j_{a v e} d B\left(1-\nu_{r \theta} \nu_{\theta r}\right)}{\left(9-\gamma^{2}\right) E_{r}} r^{3}
$$

Because neither the coil form nor overbanding carry current the particular solution in Eq. 7.18 applies only to the winding elements. The coil's structural elements simply react to the winding's applied electromagnetic forces. It should be noted that Eq. 7.18 posses singularities at both $\gamma=2$ and at $\gamma=3$; for unately, alternate version of the particular solution are readily derived by substituting the numeric values of $\gamma$ directly into Eq. 7.5. 


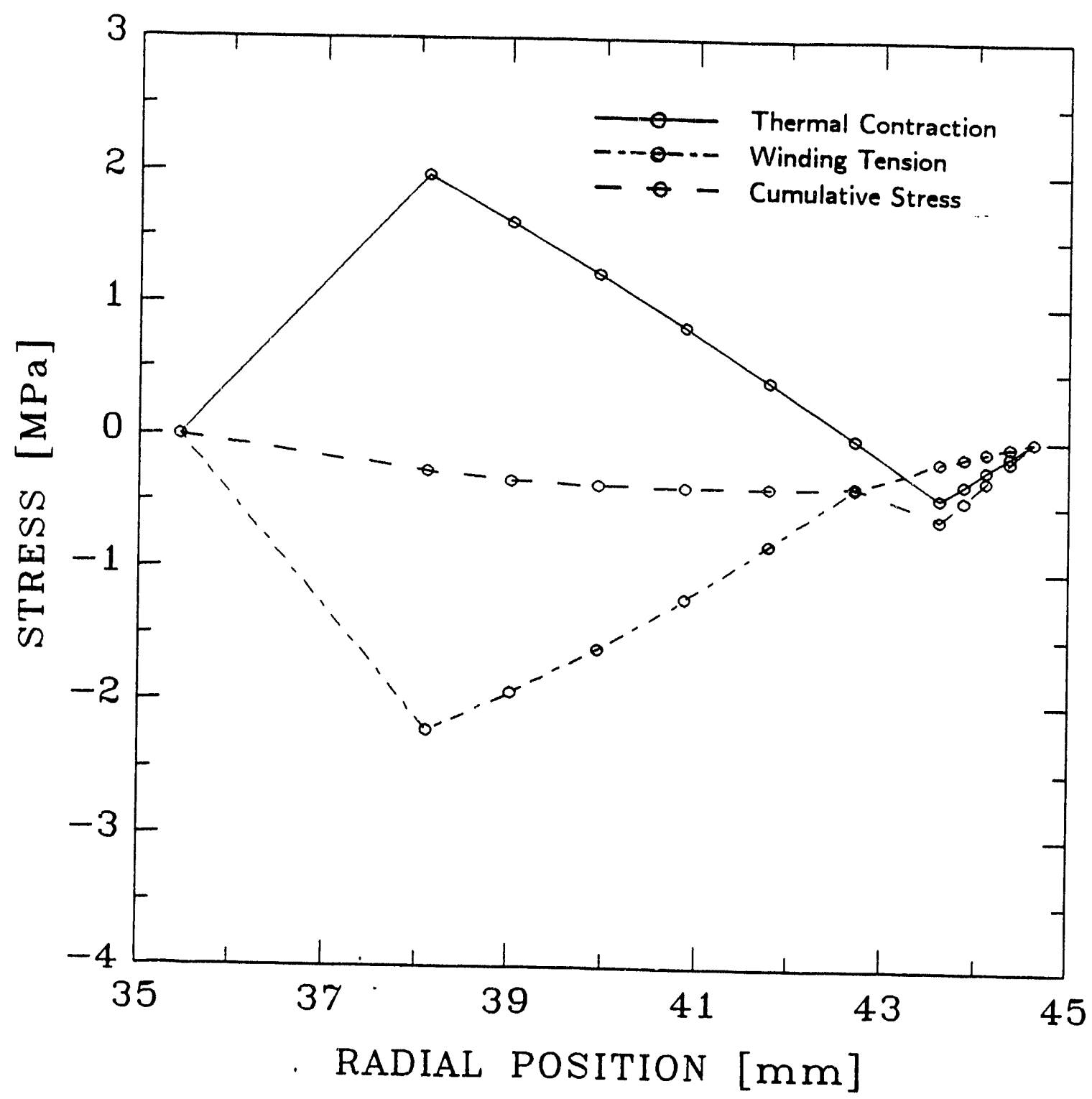

Figure 7.5: Winding-tension-induced radial stress vs radial location. Also included in this graph are traces showing the thermal-contraction stresses, and the cumulative stress state following both winding and cooldown. The open circles along the traces indicate the coil element boundaries. 
Figure 7.6 presents the cumulative radial stress distribution at the coil midplane at various points during energization. Because radial compression stresses exist at the bore tube following cooldown, radial separation between the winding and coil form is handled by applying a slight variation of Eq. 7.8. The model's electromagnetic stresses are first calculated assuming continuous radial contact between all of the coil elements. The cumulative stress distribution is then determined by superposing the residual winding stresses, thermal contraction stresses, and electromagnetic force reaction stresses. If the radial stresses remains everywhere compressive, the analysis is finished. However, if the cumulative radial stress between the coil form and winding is positive, $\sigma_{r_{0}}^{c}\left(r_{1}\right)=\sigma_{r_{1}}^{c}\left(r_{1}\right)>0$, an additional component is added to the cumulative stress; this component is computed by allowing discontinuous radial displacements at $r_{1}$ and applying the following radial stress boundary conditions:

$$
\begin{aligned}
& \sigma_{r_{0}}\left(r_{1}\right)=-\sigma_{r_{0}}^{c}\left(r_{1}\right) \\
& \sigma_{r_{1}}\left(r_{1}\right)=-\sigma_{r_{1}}^{c}\left(r_{1}\right),
\end{aligned}
$$

to produce zero net stress at the coil-form/winding interface. The superscript $c$ is used to denote the cumulative tensile stress state prior to coil-form/winding separation. After the tensile stresses responsible for winding/coil-form separation are accounted for, the radial stress distribution within the winding is rechecked to verify that its cumulative stress remains everywhere compressive. If any interconductor tensile radial stresses develop during energization either the winding tension or overbanding structure must be reconfigured to eliminate them.

\subsection{Application of electromagnetic-spring-friction force model to coil design}

A greatly simplified version of §5.2's electromagnetic friction force model was employed in the insert coil's design. For small superconducting magnets, §6.4's shows that the effective spring force of a single conductor turn is typically much smaller that its static friction force. Consequently, the simplified electromagneticfriction force model entirely neglects this spring force contribution and expresses the ratio of the forces applied to each conductor segment during energization as an 


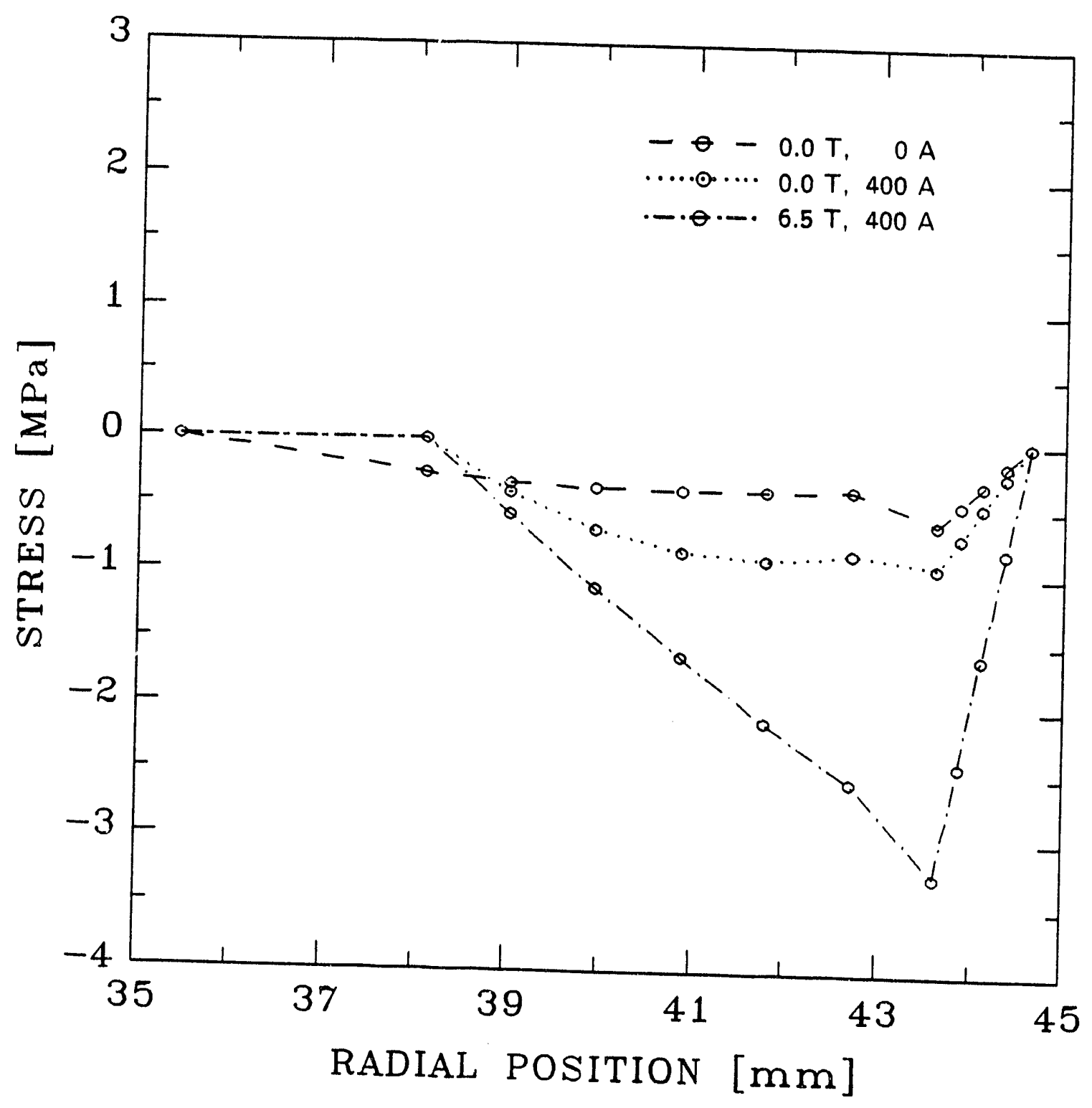

Figure 7.6: Cumulative radial stress at the insert coil midplane $v s$ radial location at various points during coil energization. The operating point in the legend indicates the insert coil current in $\mathrm{A}$ and the $6 \mathrm{~B}$-Bitter magnets central induction in $\mathrm{T}$. The open circles along the traces indicate the coil element boundaries. 
applied friction coefficient, $\mu_{a p p}$.

$$
\mu_{a p p}(i, z)=\frac{F_{z}(i, z)}{F_{r}(i, z)} \quad(i=1, \ldots, 6) ;(-63.1 \mathrm{~mm}<z<63.1 \mathrm{~mm})
$$

$F_{z}(i, z)$ is the axial electromagnetic force acting on an single conductor segment of length $\ell$, located at an axial location $z$ in the $i$ th conductor layer. The axial location, $z$, is referenced with respect to the coil's midplane.

$$
F_{z}(i, z)=I \ell B_{r}(i, z)
$$

The radial components of the magnetic induction used in this analysis are obtained using the magnetic field maps in Appendix A. The radial force on the conductor segment, $F_{r}(i, z)$, is calculated using the clumulative radial stress distribution at the axial location $z$.

$$
F_{r}(i, z)=w \ell\left(\sigma_{r_{i}}^{c}\left(r_{i}\right)+\sigma_{r_{i}}^{c}\left(r_{i+1}\right)\right)
$$

$w$ is the conductor segment's width. Given the probable existence of interconductor gaps at the start of the first charging sequence, motion is then possible when the applied friction coefficient, $\mu_{a p p}(i, z)$ exceeds the segment's static friction coefficient, $\mu_{.}$.

In a radially subdivided superconducting solenoid the inner and outer coil are typically connected in series and share a common power supply. However, because the outer coil during these tests was simulated by the $6 \mathrm{~B}$ Bitter solenoid it is experimentally difficult to energize both magnets simultaneously. Hence, each magnet was energized independently while holding the other coil's operating point fixed. Figure 7.7 represents the various energization sequences used during these tests. The line OD is the insert coil load line in a $0.0 \mathrm{~T}$ background Bitter induction. The line segments EF and BP depict the insert coil's load lines with the Bitter magnet's central induction at 3.25 and $6.5 \mathrm{~T}$ respectively. The line segment $\mathbf{A P}$ is obtained by holding the insert coil at its $400 \mathrm{~A}$ operating current and increasing the Bitter magnet's central induction from 0.0 to $6.5 \mathrm{~T}$.

The numbers listed along each of these paths denote the applied friction coefficient, $\mu_{a p p}$, at the ends of the winding's inner most conductor layer at the corresponding point in the charging sequence. This coil location is emphasized because 


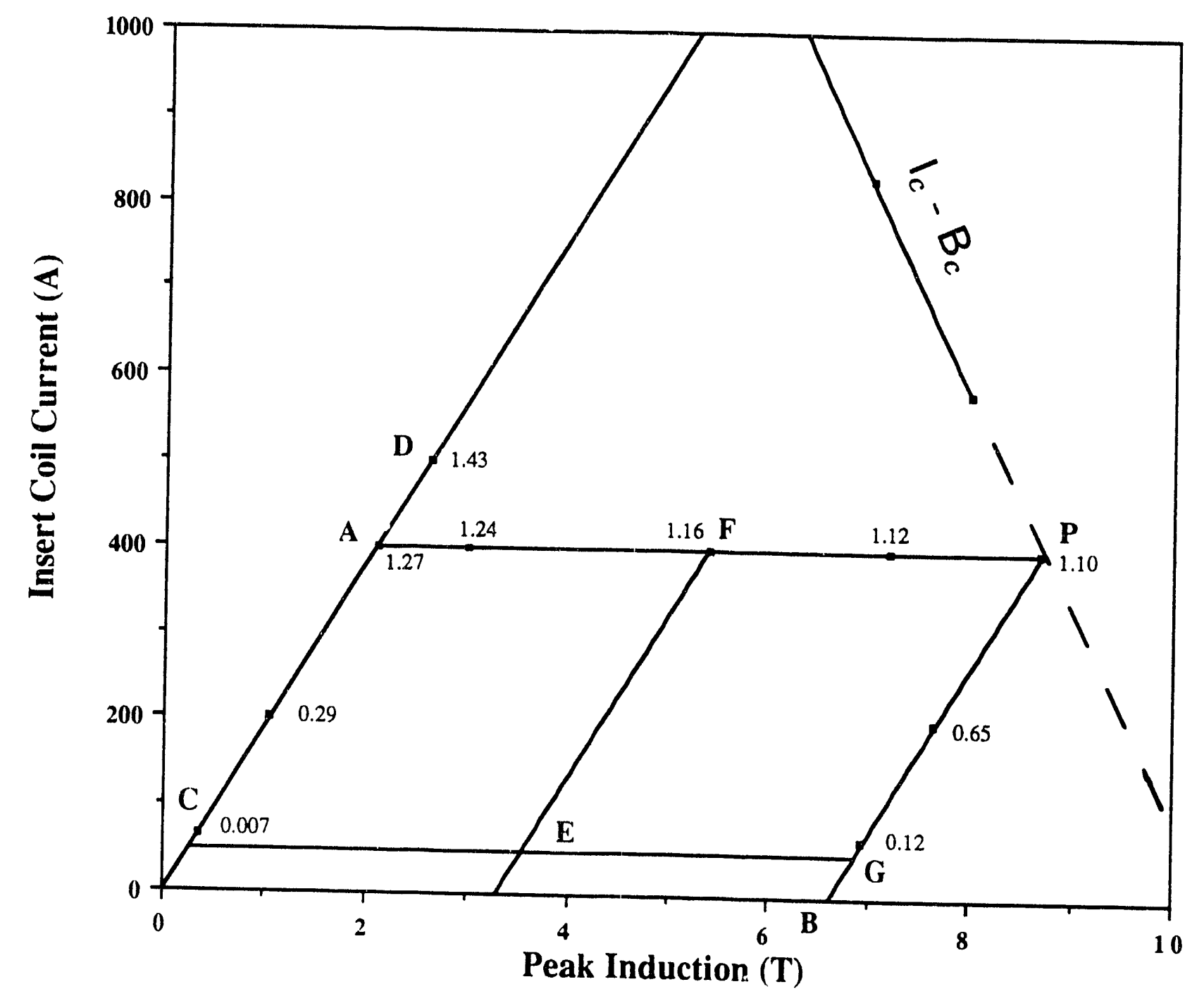

Figure 7.7: Test sequences used during insert coil energization. The numbers listed along the charging line indicate the predicted values of the friction coefficient at the end of the inner most conductor layer. 
it is frequently cited as the origin of most conductor-motion-induced quenches. The applied friction coefficient increases markedly along the path $\mathbf{0 A}$, achieving a peak value of 1.26 at point $\mathbf{A}$. $\mu_{a p p}$ decreases slightly as charging continues along path AP and settles to a value of $\sim 1.10$ at the coil approaches its critical point $\mathbf{F}$.

The model insert coil achieves the desired objective of encouraging conductor motions during low field operation where the coil's energy margin is high and of discouraging these motions as the winding nears its critical point. This design objective is obtained by combining a very low initial conductor tension with an extremely rigid external support structure provided by the four $\mathrm{BeCu}$ overbanding layers. The balance of the chapter describes the experimental program developed to test this electromagnetic-friction force model using the prototype superconducting coil described in Table 7.2. This coil was manufactured using magnet construction facilities available at the FBNML.

\subsection{Instrumentation}

Three measuring circuits were used to monitor the coil performance during energization. These circuits include: shunt resistors to measure both the insert coil and Bitter solenoid's operating currents, acoustic emissions (AE) transducers to sense abrupt mechanical disturbances, and differential voltage taps to monitor the conductor motions. The differential voltage taps were also used to determine the insert coil's radial quench initiation locations.

The shunt resistor voltages were displayed continuously during testing using digital roltmeters. These voltmeter signals were used to regulate the timing of the coils' energization sequences. Quench detection for the insert coil was provided by visually monitored the coil's terminal voltage on a cathode ray oscilloscope. In the event of a quench it is important to rapidly discharge the coil's operating current; this limits the helium boil-off and reduces the possibility of ohmic-heating-induced thermai damage. Neither the peak nor central magnetic inductions were directly measured during the experiments, rather they were inferred from the magnets' measured operating currents and the analytically determined magnet field profiles presented in Appendix A. All measuring circuit outputs were continuously recorded during energization using a 14-channel Honeywell model 5600E multiple-speed tape 
recorder. The recorded data signals were analyzed after all test runs were complete.

The combined AE/voltage monitoring method was described in $§ 3.3$ as a sensitive means for characterizing conductor motion disturbances. Two AE transducers mounted $\sim 6 \mathrm{~mm}(1 / 4 \mathrm{in})$ from each ends of the coil's outer surface were used to approximate the axial locations of the conductor motion disturbances. Figure 7.8 illustrates the details of the $\mathrm{AE}$ sensor mounting. Because the insert coil separates from it's coil form part way through its charging sequence it is desirable to mount the AE sensors to the outer surface of the overbanding; this ensures an uninterrupted transmission path between disturbance source and the sensor location. Each AE sensor's mounting arrangement consists of an aluminum bracket that firmly presses the transducer to a contoured brass base that is indium soldered to the outer surface of the $\mathrm{BeCu}$ strip. The space needed to externally mount $\mathrm{AE}$ sensors was the principal factor limiting the insert coil's outer diameter. The insert coil cryostat's inner bore is $123.8 \mathrm{~mm}$ ( $4.875 \mathrm{in}$ ) and the $\mathrm{AE}$ sensors require a radial clearance of $\sim 16 \mathrm{~mm}$ ( $5 / 8 \mathrm{in}$ ); this limits the insert coil's outer diameter to less than $\sim 92 \mathrm{~mm}$ (3.62 in). From Table 7.2, the insert coil's actual outer diameter is $89.3 \mathrm{~mm}$.

Seven voltage taps, located one to each end of the winding layers, were used to estimate the extent and approximate radial location of the conductor-motioninduced disturbances. These voltage tap signals were monitored using a previously untried data processing scheme. In this scheme pairs of adjacent conductor layers were configured into inductive $1 / 2$-bridge circuits. The intent of the scheme was to increase the resolution of the conductor-motion-induced voltage spikes by offsetting the adjacent layers' inductive charging voltages, leaving only the uncancelled voltage spike. A schematic diagram of these passive, inductive bridges is presented in Figure 7.9. The double-layer voltages from each of pair of adjacent conductors were also recorded; these double-layer voltages serve as the principal reference source for determining the magnitudes and approximate radial locations for the insert coil's conductor-motion-induced disturbances. The amplified inductive bridge readings were much noisier than anticipated, perhaps because of their limited dynamic response. Hence, the bridge measurements were only used when necessary to supplement the direct voltage determinations. 

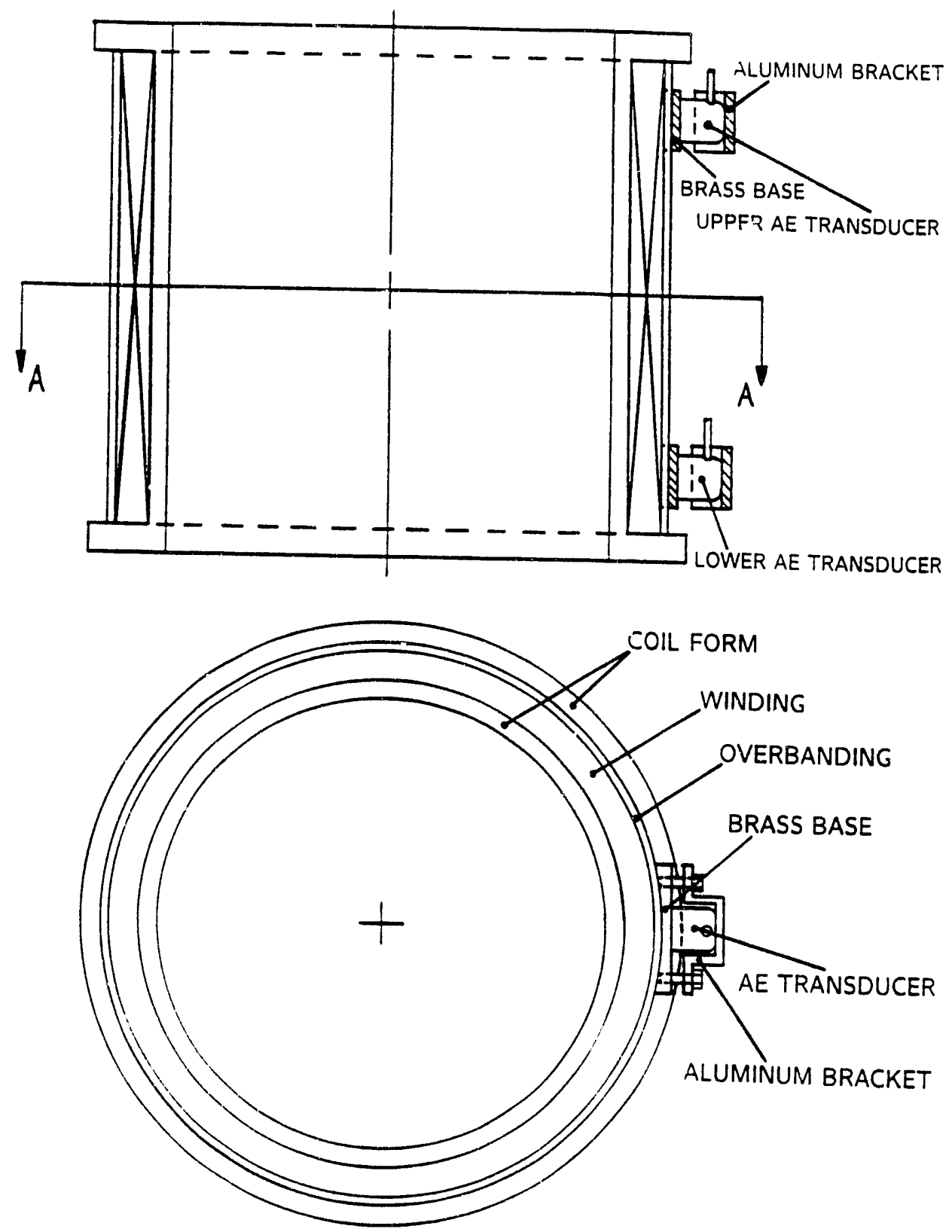

SECTION AA

Figure 7.8: Schematic diagram showing details of AE sensor mounting. 


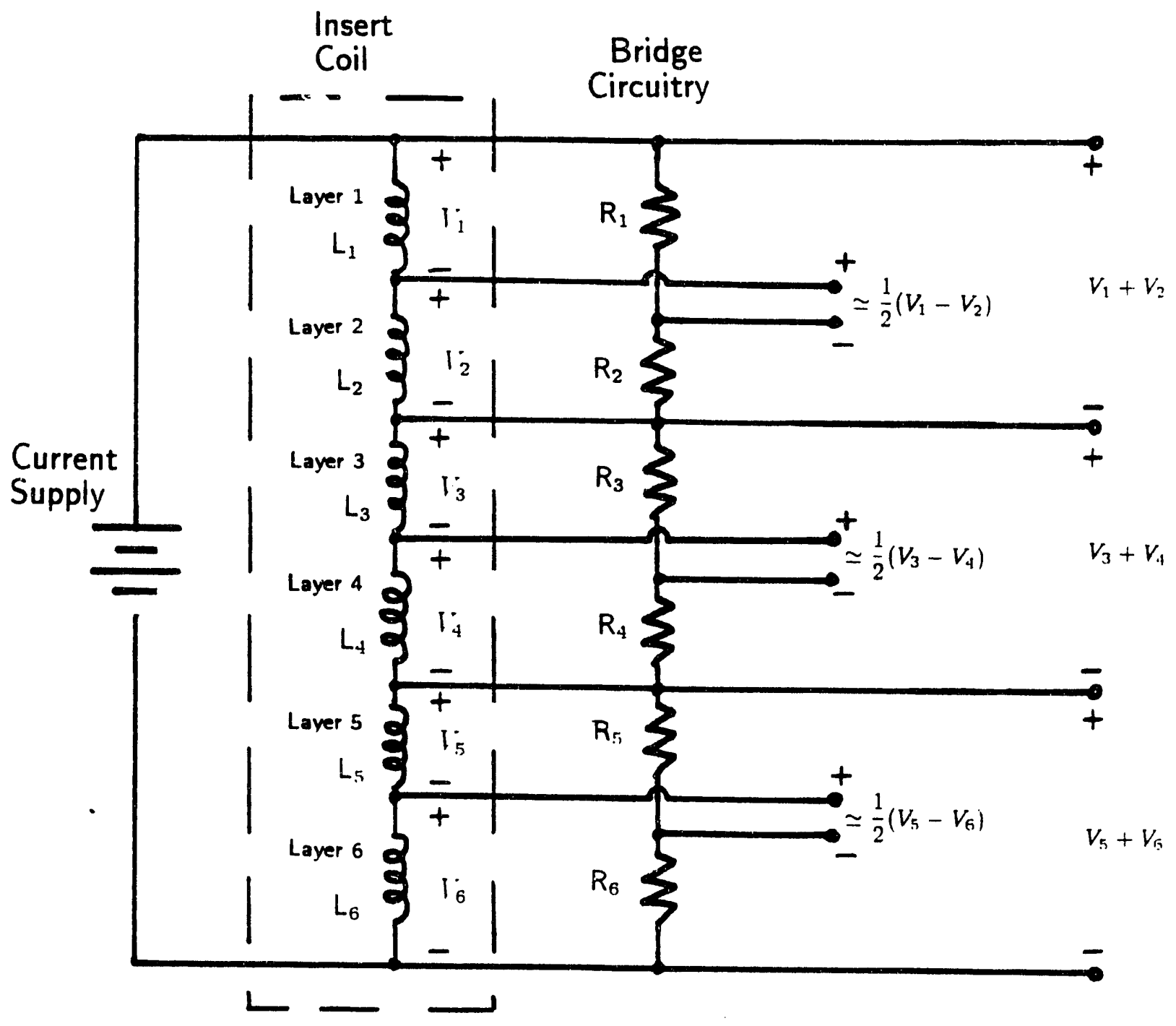

Figure 7.9: Insert coil inductive bridge circuitry. All layers have approximately equal inductances. The completion resistors are chosen so that $R_{1} / R_{2}=L_{1} / L_{2}$, $R_{3} / R_{4}=L_{3} / L_{4}$, and $R_{5} / R_{6}=L_{5} / L_{6}$. 


\subsection{Experimental Results}

\subsubsection{Energization record}

Table 7.4 summarizes the charging sequences used during each of the investigation's twelve test runs. The charging sequences are referenced with respect to the various paths depicted in Figure 7.7. Nine quenches were observed during the test sequences. The insert coil's operating current and peak magnetic induction just prior to each of these quenches are recorded in Table 7.4. The experimental evidence indicates that all nine quenches were induced by conductor motion disturbances. The last three quenches (runs 10-12) occurred at $\sim 98 \%$ of the coil's theoretical critical current. The operating conditions corresponding to each of the insert coil quenches are represented diagrammatically in Figure 7.10.

\subsubsection{Quench characterization}

Figure 7.11 presents representative $\mathrm{AE}$ /voltage traces obtained during the coil's second quench (run 5). All of the other quenches except the first one show similar characteristics. The upper trace in Figure 7.11a is from the AE transducer mounted near the upper half of the coil; the 2nd trace shows the voltage signal from the inner two conductor layers (layers $1+2$ ); the 3 rd trace, from the middle two conductor layers (layers $3+4$ ); and the 4 th trace, from the outer two layers (layers $5+6$ ). The following conductor-motion-induced quench characteristics are evident. The double layer voltage spikes coincide with an $\mathrm{AE}$ burst; this verifies the mechanical origins of the spikes. Second, there is no net change in the coil's terminal voltage during quench initiation. The voltage increase in layers $5+6$ is matched by a corresponding decrease in the inner four layers. Third, the upward voltage spike in the layers $5+6$ is followed by a rising resistive voltage; this indicates that the quench was initiated by an axial conductor motion somewhere in these two layers. Supplemental information from the inductive bridge measurements indicates that the motion occurred in the sixth layer. Figure 7.11b presents a time-expanded traces of the: (top trace) layer 1+2 voltage, (middle trace) upper $\mathrm{AE}$ signal, and (bottom trace) lower AE signal. The time offset between the AE signals, $\sim 0.1 \mathrm{~ms}$, indicates that the conductor motion occurred in the lower half of the winding.

Table 7.5 summarizes the magnitude and approximate locations for each of the 
Table 7.4: Test coil charging sequences

\begin{tabular}{|c|c|c|c|c|r|}
\hline Run & $\begin{array}{c}\text { Charging } \\
\text { Sequence }\end{array}$ & $\begin{array}{c}\text { Bitter } \\
\text { Induction, } \\
B_{0}(\mathrm{~T})\end{array}$ & $\begin{array}{c}\text { Insert } \\
\text { Current } \\
(\mathrm{A})\end{array}$ & $\begin{array}{c}\text { Combined } \\
\text { Peak Field, } \\
B_{w}(\mathrm{~T})\end{array}$ & $\begin{array}{r}\text { Quench } \\
\text { Result }\end{array}$ \\
\hline 1 & $0-\mathrm{D}$ & 0.00 & 456.0 & 2.37 & Induced \\
\hline 2 & $0-\mathrm{A}$ & 0.00 & 400.0 & 2.08 & None \\
\hline 3 & $\mathrm{~A}-\mathrm{D}$ & 0.00 & 500.0 & 2.60 & None \\
\hline 4 & $0-\mathrm{D}$ & 0.00 & 500.0 & 3.60 & None \\
& $\mathrm{D}-\mathrm{0}$ & - & - & - & None \\
\hline 5 & $0-\mathrm{A}$ & 0.00 & 402.8 & 2.08 & None \\
& $\mathrm{A}-\mathrm{P}$ & 4.48 & 402.8 & 6.65 & Induced \\
\hline 6 & $0-\mathrm{A}$ & 0.00 & 400.1 & 2.08 & None \\
& $\mathrm{A}-\mathrm{P}$ & 4.14 & 400.1 & 6.29 & Induced \\
\hline 7 & $0-\mathrm{A}$ & 0.00 & 399.7 & 2.08 & None \\
& $\mathrm{A}-\mathrm{P}$ & 6.01 & 399.7 & 8.19 & Induced \\
\hline 8 & $0-\mathrm{A}$ & 0.00 & 402.6 & 2.08 & None \\
& $\mathrm{A}-\mathrm{P}$ & 5.68 & 402.6 & 7.87 & Induced \\
\hline 9 & $0-\mathrm{A}$ & 0.00 & 399.7 & 2.08 & None \\
& $\mathrm{A}-\mathrm{P}$ & 5.94 & 399.7 & 8.12 & Induced \\
\hline 10 & $0-\mathrm{C}$ & 0.00 & 50.0 & 0.26 & None \\
& $\mathrm{C}-\mathrm{E}$ & 3.29 & 50.0 & 3.60 & None \\
& $\mathrm{E}-\mathrm{F}$ & 3.29 & 400.0 & 5.42 & None \\
& $\mathrm{F}-\mathrm{E}$ & - & - & - & None \\
& $\mathrm{E}-\mathrm{B}$ & 6.55 & 50.0 & 6.91 & None \\
& $\mathrm{B}-\mathrm{P}$ & 6.55 & 389.8 & 8.68 & Induced \\
\hline 11 & $0-\mathrm{C}$ & 0.00 & 50.0 & 0.26 & None \\
& C-B & 6.55 & 50.0 & 6.91 & None \\
& $\mathrm{B}-\mathrm{P}$ & 6.55 & 390.0 & 8.68 & Induced \\
\hline 12 & $0-\mathrm{C}$ & 0.00 & 50.0 & 0.26 & None \\
& $\mathrm{C}-\mathrm{B}$ & 6.55 & 50.0 & 6.91 & None \\
& $\mathrm{B}-\mathrm{P}$ & 6.55 & 388.7 & 8.67 & Induced \\
\hline
\end{tabular}




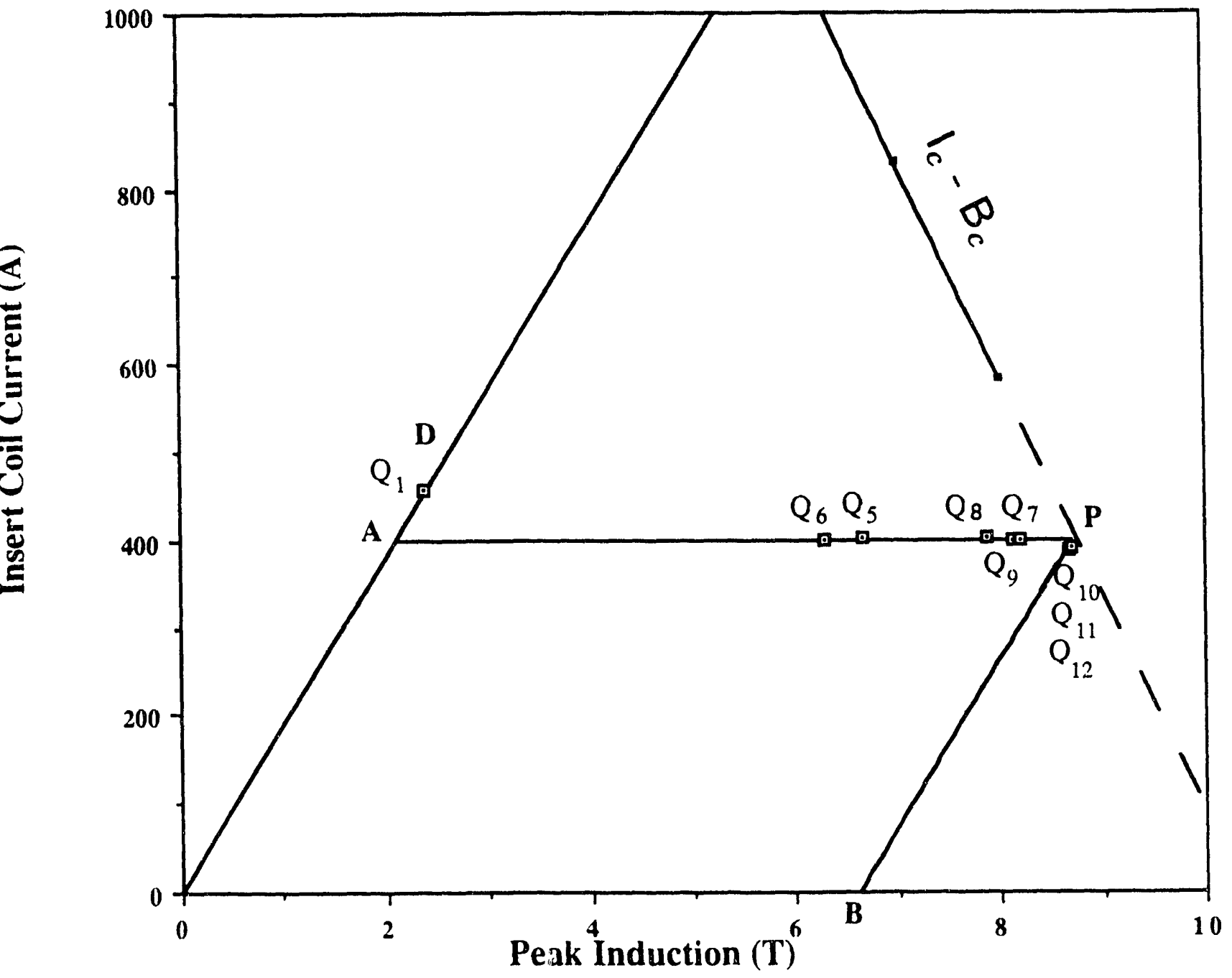

Figure 7.10: Energization diagram showing operating conditions immediately preceeding insert coil quench. Each quench is marked by symbol $Q_{i}$ where $i$ refers to the test run in which the quench occurred. 

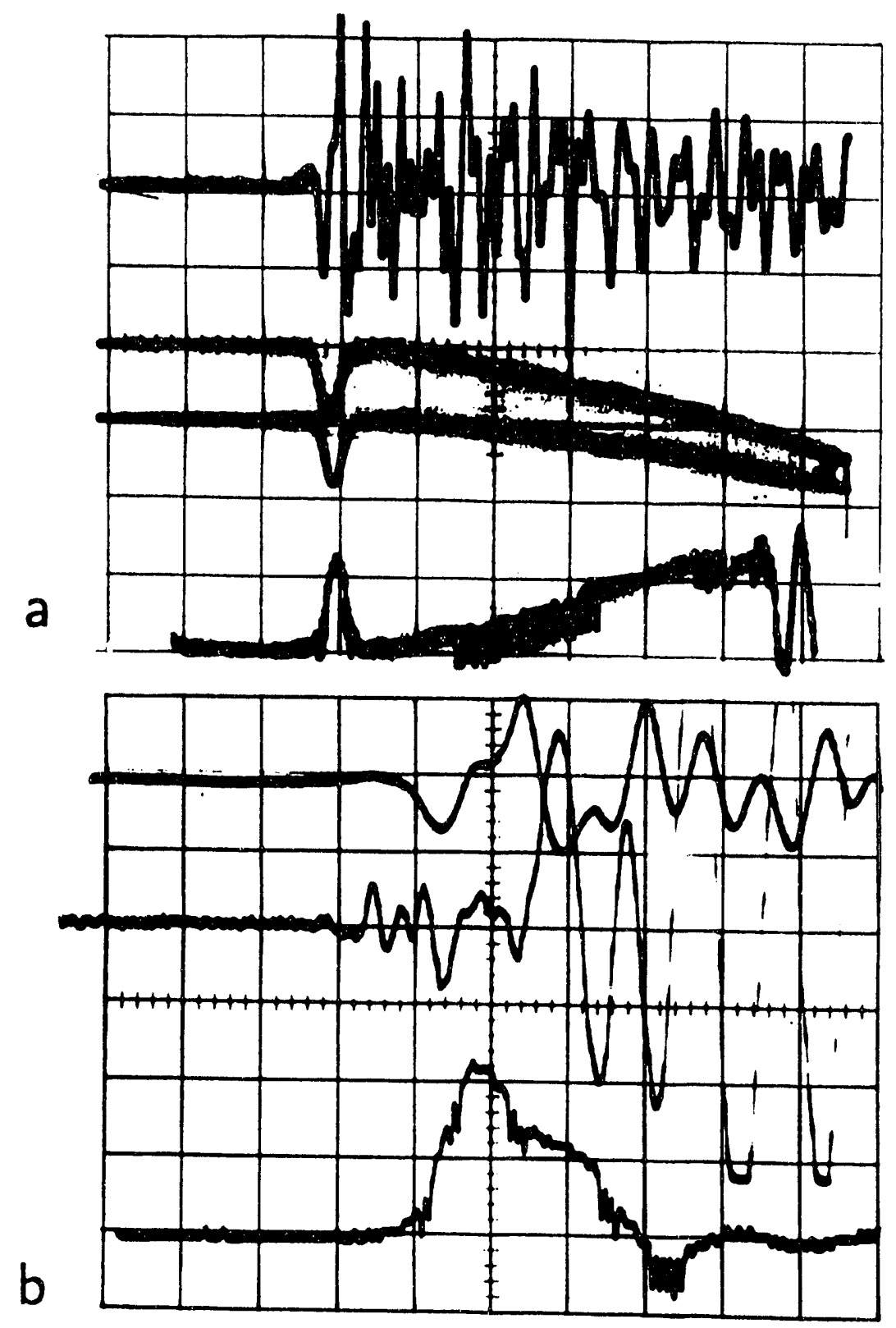

Figure 7.11: AE/voltage traces observed during second coil quench (run 5). a) Top trace: upper AE transducer $\left(5 \mathrm{~V}_{f}\right)$. Second trace: Layer $1+2$ voltage $\left(1 \mathrm{~V}_{f s}\right)$. Third trace: Layer $3+4$ voltage $\left(20 \mathrm{~V}_{f s}\right)$. Bottom trace: Layer $5+6$ voltage $\left(1 \mathrm{~V}_{f}\right)$. Time base $5.12 \mathrm{~ms} f s$. b) Top trace: Layer $1+2$ voltage $(1$ $\left.\mathrm{V}_{f_{s}}\right)$. Second trace: upper $\mathrm{AE}$ transducer $\left(5 \mathrm{~V}_{f_{s}}\right)$. Bottom trace: lower $\mathrm{AE}$ transducer $\left(5 \mathrm{~V}_{f s}\right)$. Time base: $1.024 \mathrm{~ms} f s$. 
nine conductor-motion-induced quenches. The approximate radial location of the conductor motions were determined by the occurrence of an upward voltage spike in one of the conductor layer pairs. The exact location was determined with reference to the inductive bridge measurements. The axial location of the conductor motion was determined by the time offsets between the AE signals. The duration of motion, $\tau$, corresponds to the voltage spike width. The extent of motion was estimated using Eq. 3.5:

$$
\delta_{i}=\frac{1}{B_{j} \ell} \int_{0}^{\tau} V(t) d t .
$$

The subscripts $i$ and $j$ in Eq. 3.5 refer to any two orthogonal directions in the winding. For all of the quenches except the first one $i$ corresponds to the windings axial direction, $z$, and $j$ to the windings radial direction, $r$. The extent axial motion responsible for the quenches during runs 5-12 were estimated for a single conductor turn near the end of the winding. For this turn $\ell=\pi \bar{d}, \bar{d}=0.08 \mathrm{~m}$, and $B_{r} \sim 1 \mathrm{~T}$.

Figure 7.12 shows the $\mathrm{AE} /$ voltage traces corresponding to the winding's first quench (run 1). The top trace shows the upper AE signal; the 2 nd trace, layer $1+2$ voltage; the 3 rd trace, layer $3+4$ voltage; and the 4 th trace, layer $5+6$ voltage. The double layer voltages all show virtually identical negative voltage spikes, followed by a rising resistive voltage in the winding's outer layers. The simultaneous occurrence of these negative voltage spikes could only have been caused by the outward radial expansion of some portion of the winding. Abrupt radial expansion would increase the winding's net magnetic flux and produce the observed negative voltage spikes. The radial displacement during run 1 is calculated based on the outward expansion of two adjacent turns; $\ell=2 \pi \bar{d}, \bar{d}=0.08 \mathrm{~m}$, and $B_{z} \sim 2.4 \mathrm{~T}$.

For most runs the quenches' rising resistive voltage immediately follows the voltage spike. However, in a few instances there was a slight time delay between the completion of the pulse and the start of the resistive increase. Conversely, at higher fields the quench began to propagate while the wire was still moving. Estimates of the time delay between the completion of wire motion and formation of a normal zone are included in Table 7.5.

\subsubsection{Disturbance histories}

One of the more disappointing aspects of this investigation was my inability 
Table 7.5: Magnitude and location of quench inducing conductor motions

\begin{tabular}{|c|c|c|c|c|c|}
\hline Run & $\begin{array}{c}\text { Pulse } \\
\text { Width } \\
\text { (ms) }\end{array}$ & $\begin{array}{c}\text { Peak } \\
\text { loltage } \\
(\mathrm{V})\end{array}$ & $\begin{array}{c}\text { Wire } \\
\text { Motion } \\
(\mu \mathrm{m})\end{array}$ & $\begin{array}{l}\text { Time } \\
\text { Delay } \\
\text { (ms) }\end{array}$ & $\begin{array}{l}\text { Location } \\
\text { (layer) }\end{array}$ \\
\hline 1 & 0.80 & 0.250 & 166 & 0.0 & 6 lower \\
\hline 5 & 0.32 & 0.167 & 107 & 0.28 & 6 lower \\
\hline 6 & 0.24 & 0.081 & 37 & 0.0 & 6 upper \\
\hline 7 & 0.32 & 0.047 & 22 & 0.0 & 6 upper \\
\hline $8^{*}$ & 0.20 & 0.012 & 5 & 0.0 & 5 upper \\
\hline 9 & 0.16 & 0.069 & 22 & 1.52 & 2 upper \\
\hline 10 & 0.32 & 0.029 & 18 & -0.28 & 5 lower \\
\hline 11 & 0.24 & 0.006 & 3 & 0.3 & 6 upper \\
\hline 12 & 0.13 & 0.003 & 1 & 0.0 & 4 upper \\
\hline
\end{tabular}

- Helium level possibly below top of magnet.

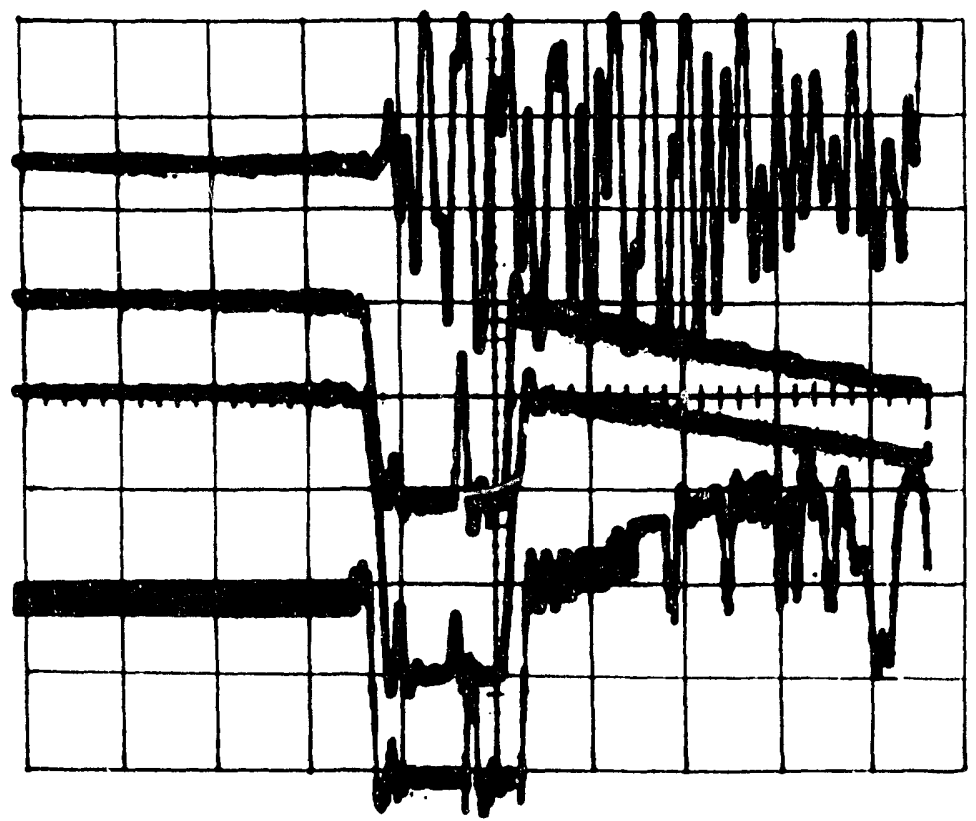

Figure 7.12: AE/voltage traces observed during first coil quench (run 1). Top trace: upper AE transducer $\left(20 \mathrm{~V}_{f s}\right)$. Second trace: Laver $1+2$ voltage $\left(1 \mathrm{~V}_{f s}\right)$. Third trace: Layer $3-4$ voltage $\left(20 \gamma_{f s}\right)$. Bottom trace: Laver $5+6$ voltage $(1$ $\left.\vee_{f s}\right)$. Time base $5.12 \mathrm{~ms} f s$. 
to produce high-resolution records of all of the conductor-motion-induced voltage spikes. The last minute substitution of a slightly less stable power supply for these experiments introduced relatively large-amplitude (compared to the voltage spikes) low frequency noise which prevented the direct translation of the tape recorded voltage signals to hard copy output. Additionally, the large number of disturbances during each test run (up to a few hundred of varying intensity) makes the manual transcription of the data traces extremely time consuming. Hence, the data analysis lacks a quantitative evaluation of the numbers, relative magnitudes, and radial distribution of conductor motions $v s$ operating conditions for each of the test runs.

My subjective interpretation of the desired disturbance histories, following prolonged visual observation of the tape recorded signals on an oscilloscope, is as follows:

1. Several tens to a few hundred AE bursts were observed during each test run. The frequency and intensity of these bursts was least near the beginning of each run. During repeated testing, the average AE burst energy decreased much more rapidly than did the number of bursts. Not all AE bursts were accompanied by obvious voltage spikes.

2. Between 50 and 150 large-amplitude $(>10 \mathrm{mV})$ voltage spikes were observed during the first few test runs. In general, the voltage spike amplitudes tended to vanishingly small values following repeated cycling.

? During the first test run these large-amplitude voltage spikes occurred with roughly equal frequency in each conductor layer. However, by the 4th test run less than $10 \%$ of the large-amplitude spikes were observed in conductor layers 1 or 2 .

\subsection{Evaluation}

A smail, dry-wound superconducting test solenoid was designed and tested. The coil demonstrated severely degraded performance during its first energization sequence. However, it trained rapidly and achieved near-critical-current performance following six training quenches. The peak field in the winding was markedly higher and the number of quenches to reach $95 \%$ critical current operation was the same, or less, than those reported in previous conductor motion investigations. $[1,2,10]$ 
The intent of the insert coil design was to configure the coil's operating stresses so as to minimize the possibility of inner layer conductor-motion-induced quenches. The experimental data (Table 7.5) confirms that none of the insert coil quenches initiated in the first conductor layer. The occurrence of several, large-amplitude conductor motions during the first few charging cycles likewise confirms that conductor motion disturbances are indeed compatible with stable low-field magnet operation.

Figure 7.13 presents a more detailed depiction of the insert coil's applied friction coefficients. Figure 7.13 plots the friction coefficient at the end of each winding layer vs the winding's peak induction. The energization sequence OAP from Figure 7.6 was assumed. The dashed horizontal line depicts Urata and Maeda's experimentally measured static friction coefficient of $\sim 0.26$ for Formvar-insulated superconductors. During the insert coil current sweep (trajectory 0A in Figure 7.6) the conductors' axial electromagnetic forces increase much more rapidly than their radial contact pressures; the applied friction coefficients increase rapidly and achieve their maximum values as the insert coil's peak induction reaches $2.1 \mathrm{~T}$. Because of the low initial winding tension the largest applied friction coefficient occurs in the first conductor layer. Alternatively, the lowest friction coefficient (highest radial pressure) occurs in the outermost conductor layer because of the high overbanding stiffness.

Contrary to the behavior observed in virtually all other dry-wound solenoids the majority of the conductor-motion-induced quenches for our test magnet occurred in the winding's outermost conductor layer; this effect is due in part to the conductor's quench energy density remaining relatively constant across the end of the winding. Figure A.3 shows that whereas the peak field in the insert coil is $8.7 \mathrm{~T}$ the total induction at the end of the conductor layers varies from $\sim 7.3 \mathrm{~T}$ at the winding's inner surface to $\sim 6.2 \mathrm{~T}$ and the outer surface. The conductor critical properties in Figure 7.3 can likewise be used to show that the field-dependent normalized operation current decreases for 0.53 at the end of the winding's inner surface to 0.4 at its outer surface. Referring back to Figure 3.1 we find that the quench energy density increases only by a factor of $2 \sim 3$ across the end of the insert coil.

The presence of a near constant quench energy density and radially varying applied friction coefficients (Figure 7.13) thus allows for a direct comparison of the relative severity of conductor motions occurring an a variety of dimensional 


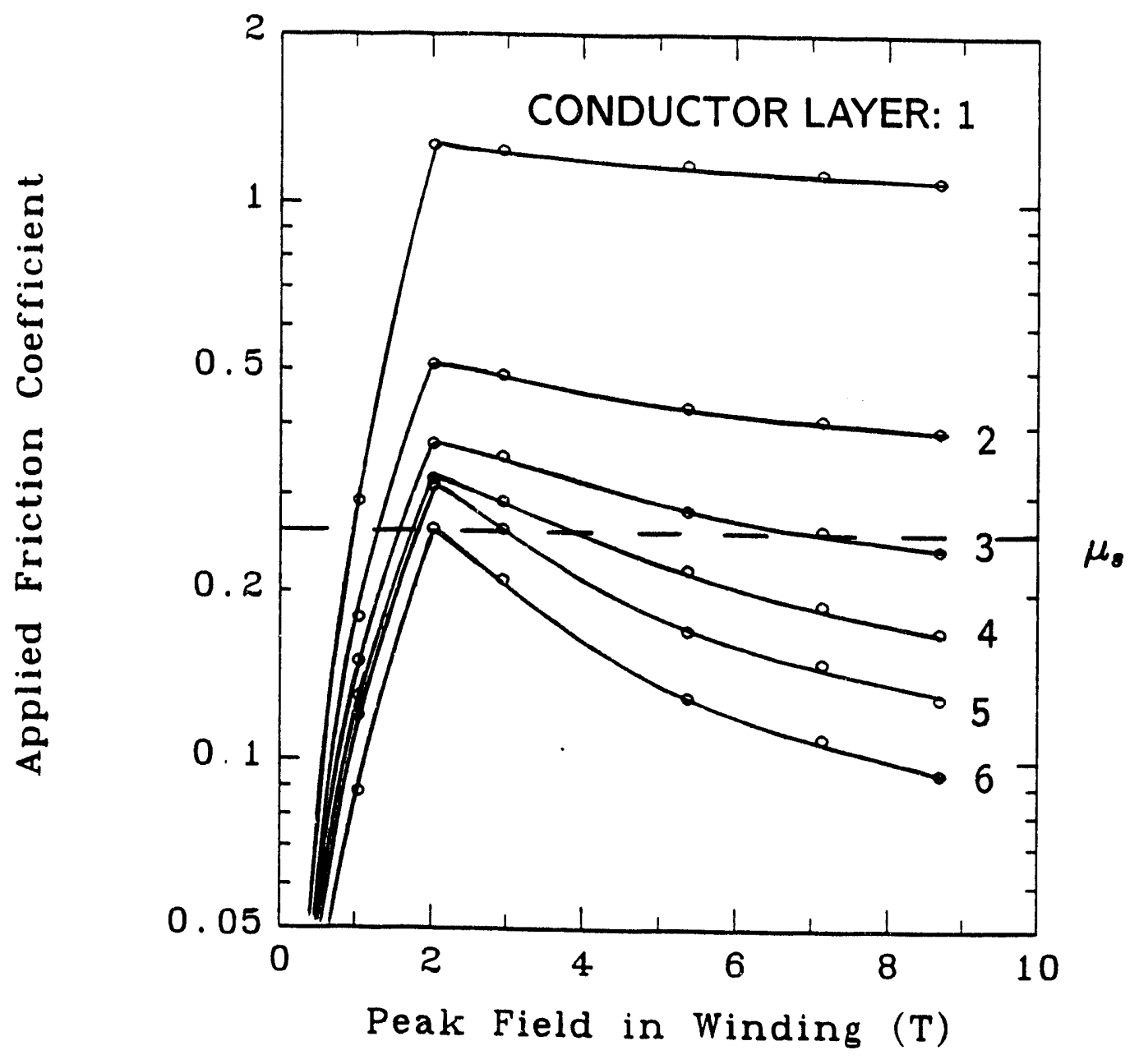

Figure 7.13: Applied friction coefficient at the end of each conductor layer vs peak field in winding. 
scales. It is interesting to note that quenching occurs most frequently in layer 6 , where the applied friction coefficient never once exceeds the conductor's static friction value. $\$ 6.4 .1$. indicates that conductor-motion disturbance occurring in the presence of low applied friction coefficients result because of the recurrent motion of poorly-supported conductor segments in an otherwise rigid winding structure. Thus, based on the insert coil's experimental results, it appears that these isolated, short conductor segment motions pose a much more serious threat to stable drywound magnet operation than the controlled low-field motion of several adjacent conductor turns.

It should be noted that the axial conductor displacements listed in Table 7.5 are approximate values based on an assumed length corresponding to a single conductor turn. The apparent displacement thus reflects the discrepancy between the actual and assumed lengths of the moving conductor segment. The very large initial conductor displacements most probable reflect the cooperative motion of several adjacent conductor turns whereas the very small motions during the later test runs are likely to involve only a fraction of a turn. The absence of large voltage spikes, especially in the inner conductor layers, after repeated charging cycles suggests the use of very large applied friction coefficients as a means for stabilizing conductormotion disturbances. By eliminating the winding's initial interconductor gaps at low stress level and low-magnetic fields, large applied friction coefficients may help to eliminate subsequent wire motions by effectively compacting the winding into a single structural unit.

In the present design the low values of $\mu_{a p p}$ in the coil's outer conductor layers occur principally because of the large overbanding thickness. The next step in the insert coil development will be to reduce, or perhaps eliminate, the overbanding thickness and redistribute the winding tension stresses so as to achieve a high applied friction coefficient throughout the winding. This insert coil design is presently under development. Test results for this magnet should be available in Proceedings of the 1992 Applied Superconductivity Conference. 


\section{REFERENCES}

1. Urata, M., and Maeda, H. Relation between radial stress and quench current for tightly wound dry solenoids IEEE Trans. MAG-23 (1987) 1596-1599.

2. Urata, M., and Maeda, H. Stabilization of superconducting dry solenoids IEEE Trans. MAG-25 (1989) 1528-1531.

3. Bobrov, E.S., and Williams, J.E.C. Direct optimization of the winding process for superconducting solenoid magnets (Linear programming approach) IEEE Trans. MAG-17 (1981) 447-448.

4. Middleton, A.J., and Trowbridge, C.W. Mechanical stress in large high field magnet coils Proc. 2nd Conf. Magn. Tech. (1967) 140-149.

5. Arp, V. Stresses in superconducting solenoids J. Appl. Phys. 48 (1977) 20262036.

6. Bobrov, E.S., and Williams, J.E.C. Stresses in superconducting solenoids Mechanics of superconducting structures, ASME applied mechanics seminar series AMD-41 (1980) 13-41.

7. Bobrov, E.S., Williams, J.E.C., and Iwasa, Y. Experimental and theoretical investigation of mechanical disturbances in epoxy-impregnated superconducting coils. 2. Shear-stress-induced epoxy fracture as the principal source of premature quenches and training - theoretical analysis. Cryogenics 25 (1985) 307-316.

8. Montgomery, D.B. Solenoid Magnet Design Wiley-Interscience, NY (1969), reprinted 1980 by R.E. Krieg $\tau$ Publishing, Malbar FL.

9. Smith, P.F., Wilson, M.N., and Spurway, A.H. Filamentary superconducting composites - IV. DC coil tests J. Fhys. D - Appl. Phys. 3 (1970) 1561-1573.

10. Schmidt, C., and Turck B. A cure against 'training' of superconducting magnets Cryogenics 17 (1977) 695-696.

11. Chikaba, J., Irie, F., Funaki, K., Takeo, M., and Yamafuji, K. Instabilities due to mechanical strain energy in superconducting magnets IEEE Trans. MAG23 (1987) 1600-1603.

12. Williams, J.E.C., and Bobrov, E.S. Magnet system of the $500 \mathrm{MHz}$ spectrometer at the Francis Bitter National Magnet Laboratory: II. Disturbances, quenches, and training Rev. Sci. Instr. 52 (1981) 657-661.

13. Cryogenic Materials Data Handbook AFML-TDR-64-280 Air Force Materials Laboratory, Wright-Patterson Air Force Base, OH (1970).

14. Koch, C.C., and Easton, D.S. A review of mechanical behaviour and stress effects in hard superconductors Cryogenics 17 (1977) 391-413. 


\section{Conclusions and recommendations}

Conductor motions are an inevitable consequence of the dry-wound construction method. Both theoretical analysis and experimental investigation indicate that abrupt motions involving $5 \sim 10 \mu \mathrm{m}$ displacements dissipate sufficient energy to induce premature quenches in high-performance superconducting magnets. These premature quenches generally occur unly during the magnet's initial charging sequences. The progressive improvement in a magnet's quench current with repeated cycling is a process kncwn as training.

Many methods have been proposed for reducing the extent of conductor-motioninduced training. The most successful method developed thus far is to eliminate the gaps between adjarent conductors using a filler like epoxy resin. However, these epoxy-impregnated solenoids are not without their difficulties. Unless fairly elaborate processing steps are taken to allow the magnet to separate from its coil form during operation, they are prone to epoxy-cracking and bore-tube-debonding disturbances.

Alternate methods for achieving reliable performance in dry-wound magnets have been examined in this thesis. The stick-slip model in Chapter 6 proposes two approaches for minimizing abrupt conductor motions. The first methods is to select superconductor-insulator pairs based on their inherently favorable frictional properties; if the conductor's slip velocity can be limited to a point at which its frictional heat generation is offset by the winding's transient thermal conduction stable performance should ensue. The second method is to alter the structural characteristics of the winding so that incipient conductor motions occur as early in the charging sequence as possible, while the magnet's quench energy margin is still relatively high.

\subsection{Frictional modification}

The basic characteristics of cryogenic polymer-metal sliding were examined in Ch. 4. It was found that whereas most polymers' indentation hardnesses increase 
markedly upon cooling to cryogenic temperatures their non-dimensional wear coefficients remain relatively constant, generally in the range $5 \times 10^{-7}-5 \times 10^{-6}$. Crossplots of the wear coefficients versus the corresponding friction coefficients indicate a third to fourth power relation between friction and wear. The magnitude of the wear coefficients and the functional relationship between friction and wear suggest that adhesive model for friction and wear remains valid description for polymeric materials at cryogenic temperatures. The identification of interfacial adhesion as a major factor in cryotribology thus allows a more systematic approach towards the investigation of sliding stability in dry-wound superconducting magnets.

The stick-slip analysis presented in Chapter 6 indicates that frictional instabilities can be eliminated through the use of inherently-stable $(\delta \mu / \delta v \gg 0)$ friction materials. Hence, the first attempt at frictional stabilization was to identify, both theoretically and experimentally, friction materials that possess, at $4.2 \mathrm{~K}$, appreciably positive friction-velocity characteristics. The adhesive friction theory suggests that positive friction-velocity characteristic's are associaced with a strain-rate-dependent interfacial shear stress. Because of the general lack of quasi-viscous creep phenomena at cryogenic temperatures, it appears that stabilization via frictional material selection is extremely unlikely for high-performance magnets.

A literature review of the sliding behaviors of contemporary magnet construction materials indicates that the commonly-used Formvar/Formvar friction pair is probably not the best possible combination for high-performance magnets. Urata and Maeda indicate that Formvar's room-temperature viscoelastic junction growth causes very unstable motion during the first conductor slip, after which its cryogenic sliding motion stabilizes considerably. This change in frictional behavior has been invoked as a partiai explanation for conductor-motion-induced training.

The adhesive friction theory suggests several alternatives for limiting this unstable firit slip characteristic. The first relies on the use of relatively inert materials to reduce the asperities' tendency towards strong interfacial bonding. A frequently used method for reducing a polymer coating's tendency towards strong adhesive is to co-polymerize the coating with about $2 \sim 5 \%$ of a perfluorocarbon intermediate to reduce its surface energy. ${ }^{[1]}$ The perfluorocarbon intermediates typically migrate to the surface of the coating, thus providing a low-friction surface layer, while leaving 
the balance of the coating's bulk mechanical properties relatively intact.

A second method for reducing the tendency towards unstable slip is to minimize transient interfacial junction growth. For instance, flexible ceramics and cuprous sulphide coatings have been examined as insulators for wind and react $\mathrm{Nb}_{3} \mathrm{Sn}$ conductors. ${ }^{[2,3]}$ Because the softening point for these insulators is markedly higher than that for conventional polymeric insulators, their tendency towards transient room-temperature creep should be greatly reduced. Hence, these inorganic insulators offer the potential advantages of being harder, more creep resistant, and less surface active than conventional polymer-based coatings; these factors should all contribute to more stable sliding initiation. An added advantage to inorganic insulators is that their transverse thermal conductivity is at least an order of magnitude larger than that for a conventional organically insulated wire. ${ }^{[4]}$ This higher transverse conductivity should further improve stability by increasing the minimum propagating zone volume, and hence, the coil's minimum quench energy.

\subsection{Structural stabilization}

A second approach towards frictional stabilization is to use the conductor tension during manufacture to regulate the winding's effective axial stiffness. Abrupt conductor motions can occur on a variety of dimensional scales. In tightly-wound solenoids, the high static friction forces provided by the conductor tension effectively pin the conductors in their as-wound positions. However, statistical variations in the conductor thickness produce short segments that are not well supported. Because of their moderate stiffness these segments frequently shift back-and-forth between equilibrium positions as the magnet is charged and discharged. The recurrent motion of these segments during magnet training frequently prevents the magnet from ever reaching its designed operating current.

The effective axial stiffness of the individual turns in a loosely wound magnet are quite low in comparison. By proper preprogramming of the winding tension, the conductor turns can be made to shift to more stable equilibrium positions while the mignet is far from its critical surface. Because their axial stiffnesses are so low, the turns tend to retain their new equilibrium positions during subsequent charging cycles, hence, loose-wound magnets tend to train progressively as the conductors 
gradually shift to a more stable winding arrangement. As the interconductor gaps are removed from the winding its effective axial stiffness increases to a level comparable with that of a similarly configured epoxy-impregnated winding. The winding approach advocated in Chapter 7 aims to achieve impregnated winding reliability in a dry-wound magnet by allowing the winding to seek a natural, electromagneticallycompacted shape from the start of its energization cycle.

The conductor motion model proposed in Chapter 7 uses a single parameter, namely the applied friction coefficient, $\mu_{a p p}$, defined as the ratio of the conductor's axial electromagnetic to radial contact forces, to estimate the extent of motion and probable training behavior in a dry-wound solenoid. Long-range conductor motions leading to progressive training are generally observed when the applied friction coefficient exceeds the conductor's static friction coefficient. Isolated conductor segments motions leading to degraded coil performance are often observed when the applied friction is less than the conductor's static friction. The entire range of friction behaviors was examined in the insert coil tested in Chapter 7. Contrary to the behavior typically observed for dry-wound solenoids, none of the coil quenches originated in the inner most conductor layer where the applied friction coefficient was greatest. Rather, the quenches originated most frequently in the outer most conductor layer where the applied friction coefficient never once exceeded the static friction value.

Additional coil tests are planned to further verify these structural tendencies. Two coil will be constructed: one whose applied friction coefficient remains everywhere below the static friction value, and one whose applied friction rises to above the static value while the insert coil current is less than half way to its designed operating limit. Results for these coil tests should appear in Proceedings of the 1992 Applied Superconductivity Conference, to be published in 1993. 


\section{References for Chapter 8}

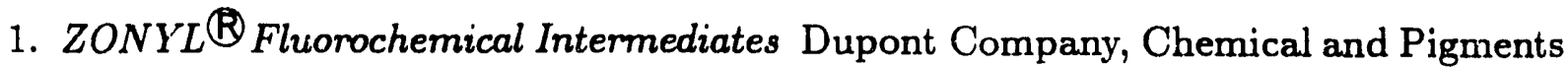
Department publication H-00172 (1988).

2. Wagner, G.R., Vecchio, P.D., and Uphoff, J.H. Cuprous sulfide as a film insulation for superconductors Adv. Cryo. Eng. 28 (1982) 821-831.

3. Holmes, D.S. The Thermal Effectiveness of Dielectric Insulations for Superconductors Technical Document AFWAL-TR-84-2008 Wright-Patterson Airforce Base, OH (1985).

4. Wilson, M.N. Stabilization, protection, and current density: some general observations and speculations Cryogenics 31 (1991) 499-503. 


\section{Appendix A. Magnetic field maps.}

Figures A.1, A.2, and $\Lambda .3$ show axial and radial components of the magnetic induction in the insert coil developed in Ch. 7 at various axial and radial locations, during different stages of its energization sequence. Because of the solenoids' circular symmetries the inductions are constant in the coil's circumferential, $\theta$, direction. Similarly, only the top half of the coil is shown because the magnitudes of the magnetic inductions are symmetric across the coil's midplane. The radial magnetic induction is odd symmetric, that is, its sign changes from the top half to the bottom half of the coil. The axial magnetic induction retains the same sign in both coil halves.

Figure A.1 shows the magnetic inductions produced solely by the insert coil at its $400 \mathrm{~A}$ operating current. These values were computed using the FORTRAN program SOLDESIGN. SOLDESIGN is a general purpose program for calculating the electromagnetic characteristics for a system of uniform current density solenoids. It was developed by Bob Pillsbury of MIT's Plasma Fusion Center (PFC) and is freely available for use on the PFC's VAX computer system where the numerical analysis for Ch. 7 was performed.

Figure A.2 shows the magnetic inductions produced solely by the $6 \mathrm{~B}-\mathrm{Bitter}$ solenoid at a 20,000 A operating current. The magnetic inductions in Figure A.2 were extracted from a graphical solution produced by Bob Weggel of the FBNML's magnet development group. This original field map shows the distributions of the magnetic inductions in the bore of the $6 \mathrm{~B}$ solenoid.

Figure A.3 shows the combined magnetic inductions produced by the insert coil at its 400 operating current and the $6 \mathrm{~B}-\mathrm{Bitter}$ solenoid at a $20,000 \mathrm{~A}$ operating current. 

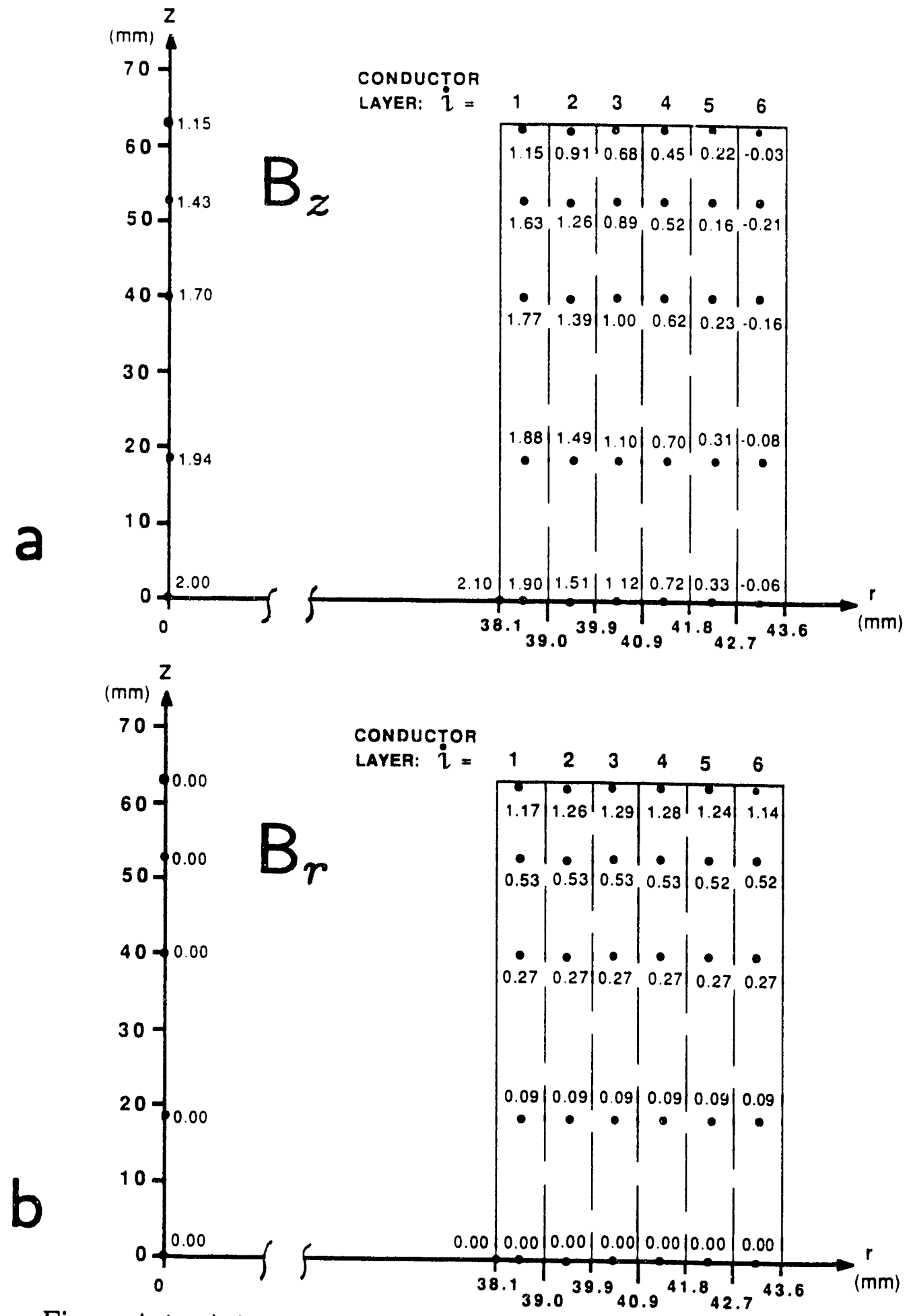

Figure A.1: a) Axial and b) Radial components of the magnetic induction in $\mathrm{T}$ at various locations in the insert coil. The magnetic induction is produced by a $400 \mathrm{~A}$ insert coil current combined with a $0.0 \mathrm{~T}$ background central induction provided by the $6 \mathrm{~B}$-Bitter solenoid. 

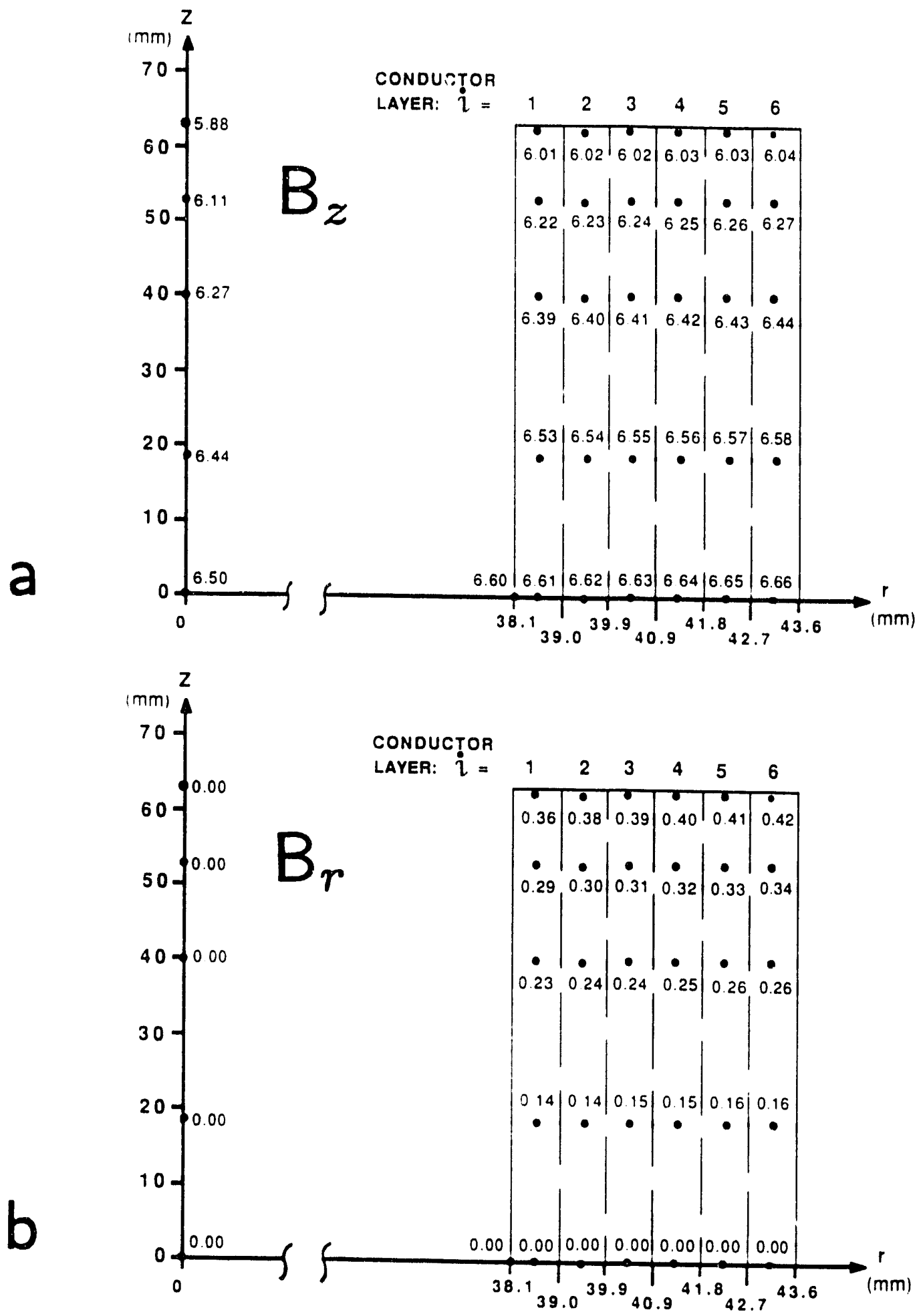

Figure A.2: a) Axial and b) Radial components of the magnetic induction in $\mathrm{T}$ at various locations in the insert coil. The magnetic induction is produced by a $0.0 \mathrm{~A}$ insert coil current combined with a $6.5 \mathrm{~T}$ background central induction provided by the $6 \mathrm{~B}-\mathrm{Bitter}$ solenoid. 

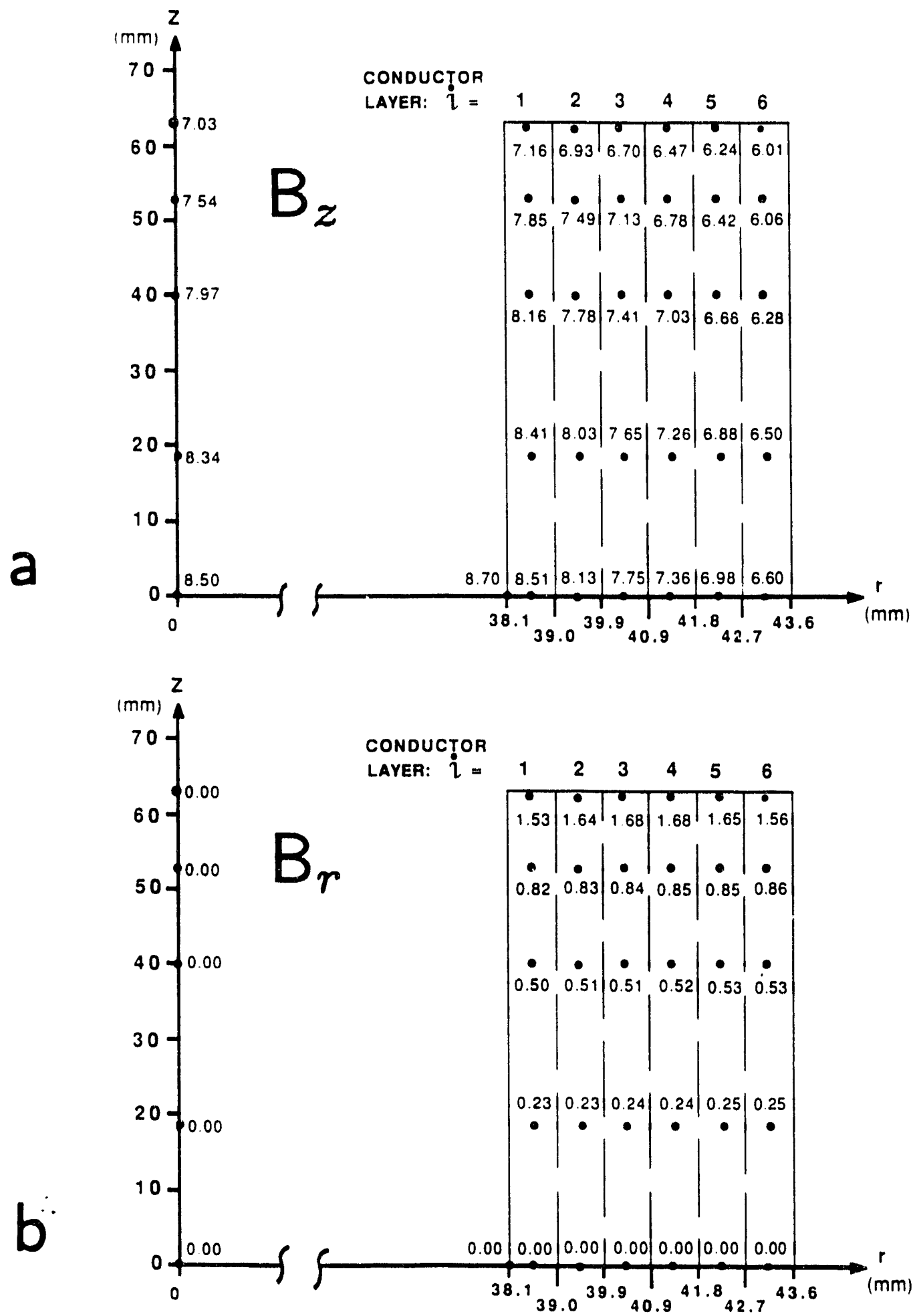

Figure A.3: a) Axial and b) Radial components of the magnetic induction in $\mathrm{T}$ at various locations in the insert coil. The magnetic induction is produced by a $400 \mathrm{~A}$ insert coil current combined with a $6.5 \mathrm{~T}$ background central induction provided by the $6 \mathrm{~B}$-Bitter solenoid. 


\section{Appendix B. Derivation of orthotropic winding properties}

The winding, or conductor portion, of a superconducting magnet generally consists of four components: superconductor, copper stabilizer, organic insulation, and void spaces. The mechanical and thermal properties of each component are reasonably well known in the range between room and liquid helium temperatures. By contrast, the aggregate properties a superconducting windings can vary over a broad range because of variations in the arrangements and relative concentrations of these components. Because of the wide variation in winding properties between magnets these properties are not generally measured, but estimated using a simple rule of mixtures analysis. In particular, whereas each winding constituent exhibits isotropic properties, the winding as a whole typically demonstrated orthotropic material behavior.

This appendix describes the algorithm used to derive the orthotropic winding properties used during the mechanical stress analysis presented in Ch. 7. Because the insert coil was constructed from rectangularly cross-sectioned conductors the concentration and arrangement of the winding's constituents are essentially the same as that in the conductor; the analysis presupposed the existence of perfectlyspace-filling, rectangular conductors. The model is derived using a 1/4-section conductor segment. The conductor's axis lies along the winding's circumferential, $\theta$, direction; its width is in the $z$ direction; and thickness in the $r$ direction. A large winding radius relative to the conductor thickness is assumed, that is, the model describes a quasi-rectilinear conductor segment.

Figure B.1 presents the seven-degree-of-freedom conductor model used to estimate the winding's orthotropic properties at 293 and $4.2 \mathrm{~K}$. The multifilamentary wire is assumed to consist of a rectangular core of superconductor surrounded by rectilinear shells of copper and insulation. The core and uninsulated wire dimensions are geometrically similar. The copper and insulation shells have each been divided into three subsections thus creating a seven-element model. The physical 


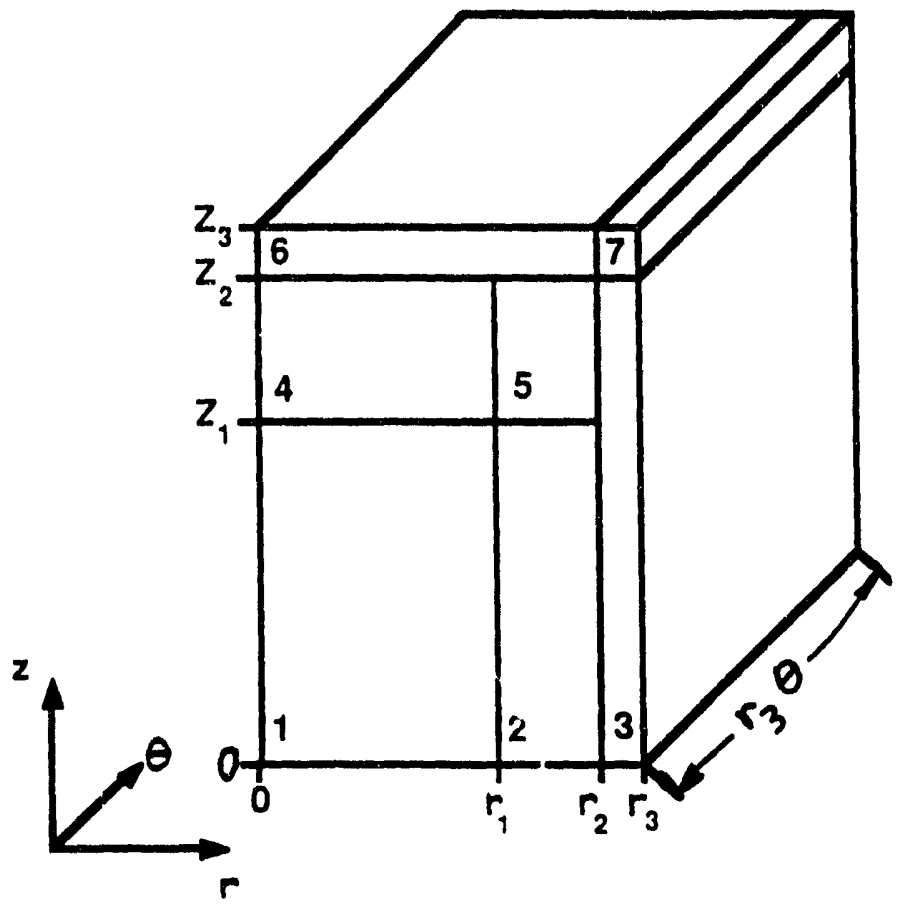

Figure B.1: Model conductor used for evaluating the insert coil's orthotropic mechanical properties.

Table B.1: Physical Dimensions for the NbTi Winding Model.

\begin{tabular}{|c|l|}
\hline \multicolumn{2}{|c|}{ Actual Conductor } \\
\hline Uninsulated conductor width & $1.215(\mathrm{~mm})$ \\
\hline Uninsulated conductor thickness & $0.859(\mathrm{~mm})$ \\
\hline Copper:superconductor ratio & 0.98 \\
\hline Insulated conductor width & $1.262(\mathrm{~mm})$ \\
\hline Insulated conductor thickness & $0.919(\mathrm{~mm})$ \\
\hline \multicolumn{2}{|c|}{ Model Conductor } \\
\hline Superconductor width, $2 z_{1}$ & $0.864(\mathrm{~mm})$ \\
\hline Superconductor thickness, $2 r_{1}$ & $0.610(\mathrm{~mm})$ \\
\hline Uninsulated conductor width, $2 z_{2}$ & $1.215(\mathrm{~mm})$ \\
\hline Uninsulated conductor thickness, $2 r_{2}$ & $0.859(\mathrm{~mm})$ \\
\hline Copper:superconductor ratio & 0.98 \\
\hline Insulated conductor width, $2 z_{3}$ & $1.262(\mathrm{~mm})$ \\
\hline Insulated conductor thickness, $2 r_{3}$ & $0.919(\mathrm{~mm})$ \\
\hline Length, $r_{3} \theta$ & $1.000(\mathrm{~mm})$ \\
\hline
\end{tabular}


dimensions for the actual. and model conductors are listed in 'lable B.1.

'The constitutive equations for cach model components, $i$, are given by:

$$
\begin{aligned}
& \left.\sigma_{r,} \quad\left(1, \nu_{i}\right)\left(1 \quad 2 \nu_{i}\right) \mid\left(1-\nu_{i}\right) c_{r_{1}} \cdot \nu_{i}\left(c_{\theta_{1}}+\boldsymbol{\epsilon}_{z_{1}}\right)\right\} \\
& \sigma_{\theta_{i}}=\frac{E_{i}}{\left(1+\nu_{i}\right)\left(1-2 \overline{\left.\nu_{i}\right)}\right.}\left[\left(1-\nu_{i}\right) \varsigma_{\theta_{1}}+\nu_{i}\left(\epsilon_{z_{1}}+\epsilon_{r_{i}}\right)\right] \\
& \sigma_{z_{2}}=\frac{E_{i}}{\left(1+\nu_{i}\right)\left(1-2 \nu_{i}\right)}\left[\left(1-\nu_{i}\right) \epsilon_{z_{1}}+\nu_{i}\left(\epsilon_{r_{1}}+\epsilon_{\theta_{i}}\right)\right] .
\end{aligned}
$$

'Table B.2 lists the conductor constituents' properties at $293 \mathrm{~K}$ and $4.2 \mathrm{~K}$. The values were obtained from the published literature. ${ }^{|1-5|}$

Table B.2: Constituent Properties for Model Conductor.

\begin{tabular}{|l|l|c|c|}
\hline $\begin{array}{l}\text { Conductor } \\
\text { Constituent }\end{array}$ & $\begin{array}{l}\mathrm{NbTi} \\
\text { Superconductor }\end{array}$ & $\begin{array}{l}\text { Copper } \\
\text { Stabilizer }\end{array}$ & $\begin{array}{l}\text { Formvar } \\
\text { Insulation }\end{array}$ \\
\hline Model Element \# & 1 & $2,4,5$ & $3,6,7$ \\
\hline $\begin{array}{l}\text { 293 K Elastic } \\
\text { Modulus (GPa) }\end{array}$ & 80 & 110 & 4 \\
\hline $\begin{array}{l}293 \mathrm{~K} \text { Poisson's's } \\
\text { Ratio, } \nu\end{array}$ & 0.33 & 0.33 & 0.33 \\
\hline $\begin{array}{l}4.2 \mathrm{~K} \text { Elastic } \\
\text { Modulus (GPa) }\end{array}$ & 80 & 117 & 8 \\
\hline $\begin{array}{l}4.2 \mathrm{~K} \text { Poisson's's } \\
\text { Ratio, } \nu\end{array}$ & 0.33 & 0.33 & 0.33 \\
\hline $\begin{array}{l}293-4.2 \mathrm{~K} \text { Thermal } \\
\text { Contraction }\end{array}$ & -0.000185 & -0.000330 & -0.001200 \\
\hline
\end{tabular}

The winding model's equivalent orthotropic properties are determined by first applying test forces to the model's various conductor faces. Assuming perfect bonding along the interfaces, the relative displacements for each component's boundaries are determined using a computer-based matrix algorithm. The relative displacements at the model's outer surfaces are then equated with those of a homogeneous body subject to the same load conditions to ac rmine the corresponding material property under consideration. This process is repeated in each direction to determine all of the directional material properties.

Table B.3 lists the seven unknown strains/relative-displacements determined by the computer program. The seven force-equilibrium equations used to derive the 
matrix algorithm are listed in Eq. B.2.

$$
\begin{aligned}
& F_{r}^{*} \quad \sigma_{r_{1}} A_{r_{1}}+\sigma_{r_{4}} A_{r_{4}}+\sigma_{r_{6}} A_{r_{6}} \\
& F_{r}^{*} \quad \sigma_{r_{2}} A_{r_{2}}+\sigma_{r_{s}} A_{r_{3}}+\sigma_{r_{6}} A_{r_{6}} \\
& F_{r}^{\prime *}-\sigma_{r_{3}} A_{r_{3}}+\sigma_{r_{7}} A_{r_{7}} \\
& F_{\theta}^{*}=\sigma_{\theta_{1}} A_{\theta_{1}}+\sigma_{\theta_{2}} A_{\theta_{2}}+\sigma_{\theta_{3}} A_{\theta_{3}}+ \\
& \sigma_{\theta_{4}} A_{\theta_{4}}+\sigma_{\theta_{5}} A_{\theta_{5}}+\sigma_{\theta_{6}} A_{\theta_{6}} ; \sigma_{\theta_{7}} \cdot A_{\theta_{7}} \\
& F_{z}^{*}=\sigma_{z_{1}} A_{z_{1}}+\sigma_{z_{2}} A_{z_{2}}+\sigma_{z_{3}} A_{z_{3}} \\
& F_{z}^{*}=\sigma_{z_{4}} A_{z_{4}}+\sigma_{z_{5}} A_{z_{6}}+\sigma_{z_{3}} A_{z_{3}} \\
& F_{z}^{*}=\sigma_{z_{6}} A_{z_{6}}+\sigma_{27} A_{z_{7}}
\end{aligned}
$$

The starred forces, $F_{r}^{*}, F_{\theta}^{*}$, and $F_{z}^{*}$ represent uniform test pressures that are applied to the model's respective outer faces. The subscripted direction is normal to the applied face. The $A_{j}$, terms likewise indicate the surface areas of element $i$ normal to the $j$ direction. The implementation of the matrix solution proceeds by substituting the unknown strains from Table B.3 into Eq. B.1, substituting Eq. B.1 into B.2, sequentially applying the test forces, and interpreting the corresponding displacements in terms of a corresponding homogeneous orthotropic model subjected to the same test force.

Table B.3: Unknown Strains.

\begin{tabular}{|l|c|c|c|}
\hline $\begin{array}{l}\text { Conductor } \\
\text { Element \# }\end{array}$ & $\epsilon_{r_{1}}=$ & $\epsilon_{\theta_{1}}=$ & $\epsilon_{z_{1}}=$ \\
\hline 1 & $\delta\left(r_{1}\right) / r_{1}$ & $\delta\left(r_{3} \theta\right) / r_{3} \theta$ & $\delta\left(z_{2}\right) / z_{2}$ \\
\hline 2 & $\delta\left(r_{2}-r_{1}\right) / r_{2}-r_{1}$ & $\delta\left(r_{3} \theta\right) / r_{3} \theta$ & $\delta\left(z_{2}-z_{1}\right) / z_{2}-z_{1}$ \\
\hline 3 & $\delta\left(r_{3}-r_{2}\right) / r_{3}-r_{2}$ & $\delta\left(r_{3} \theta\right) / r_{3} \theta$ & $\delta\left(z_{3}-z_{2}\right) / z_{3}-z_{2}$ \\
\hline 4 & $\delta\left(r_{1}\right) / r_{1}$ & $\delta\left(r_{3} \theta\right) / r_{3} \theta$ & $\delta\left(z_{1}\right) / z_{1}$ \\
\hline 5 & $\delta\left(r_{2}-r_{1}\right) / r_{2}-r_{1}$ & $\delta\left(r_{3} \theta\right) / r_{3} \theta$ & $\delta\left(z_{2}-z_{1}\right) / z_{2}-z_{1}$ \\
\hline 6 & $\delta\left(r_{2}\right) / r_{2}$ & $\delta\left(r_{3} \theta\right) / r_{3} \theta$ & $\delta\left(z_{2}\right) / z_{2}$ \\
\hline 7 & $\delta\left(r_{3}-r_{2}\right) / r_{3}-r_{2}$ & $\delta\left(r_{3} \theta\right) / r_{3} \theta$ & $\delta\left(z_{3}-z_{2}\right) / z_{3}-z_{2}$ \\
\hline
\end{tabular}

For instance, the radial modulus, $E_{r}$, is determined by numerically applying the following test forces to the matrix model; $F_{r}^{*}=1 ; F_{\theta}^{*}=0$; and $F_{z}^{*}=0$. By symmetry, the model's midplanes remains stationary, this is the reason only a $1 / 4$ section model was used. The combined total strains, $\epsilon_{r}^{c}=\delta\left(r_{3}\right) / r_{3}, \epsilon_{\theta}^{c}=\delta\left(r_{3} \theta\right) / r_{3} \theta$, 
and $\iota z \delta\left(z_{3}\right) / z_{3}$ are then determined and compared to a homogeneous body subject to the same test force. The radial modulus is determined by: $E_{r} \quad \sigma_{r}^{*} / e_{r}^{r}$. where $\sigma_{r}^{*}$ is the normal pressure corresponding to $F_{r}^{*}$. The transverse Poisson's ratios are likewise calculated from the ratios of the lateral to direct deformations. $\nu_{\theta r}-\epsilon_{i}^{c} / \epsilon_{r}^{r}$, and $\nu_{z r}=\epsilon_{z}^{c} / \epsilon_{r}^{c}$. Table B.4 presents the winding model's equivalent 4.2- and 293-K orthotropic mechanical properties.

Table B.4: Equivalent Orthotropic Mechanical Properties.

\begin{tabular}{|l|c|c|}
\hline & at $293 \mathrm{~K}$ & at $4.2 \mathrm{~K}$ \\
\hline $\begin{array}{l}\text { Radial Modulus, } \\
\mathrm{E}_{r}(\mathrm{GPa})\end{array}$ & 45.9 & 63.0 \\
\hline $\begin{array}{l}\text { Circumferential } \\
\text { Modulus, } \mathrm{E}_{\theta}(\mathrm{GPa})\end{array}$ & 85.8 & 89.3 \\
\hline $\begin{array}{l}\text { Axial Modulus, } \\
\mathrm{E}_{z}(\mathrm{GPa})\end{array}$ & 57.3 & 71.9 \\
\hline$\nu_{r \theta}$ & 0.33 & 0.33 \\
\hline$\nu_{\theta z}$ & 0.22 & 0.27 \\
\hline$\nu_{z r}$ & 0.18 & 0.24 \\
\hline$\nu_{\theta r}$ & 0.18 & 0.23 \\
\hline$\nu_{z \theta}$ & 0.33 & 0.33 \\
\hline$\nu_{r z}$ & 0.23 & 0.27 \\
\hline
\end{tabular}

The winding's orthotropic thermal contractions are determined in an analogous manner. However, in this case no test forces are used. Rather, the relative displacements at the interfaces between conductor elements are expressed as elastic strains combined with the components' known thermal contractions. Static equilibrium is obtained by equating the thermal contraction induced stresses with the model's resulting internal elastic stresses. Table B.5 presents the winding model's orthotropic thermal contractions.

Table B.5: Equivalent Orthotropic Thermal Contractions.

\begin{tabular}{|c|c|}
\hline 293-4.2 K Radial & -0.003770 \\
\hline Thermal Contraction, $\Delta_{r}$ & \\
\hline $\begin{array}{c}\text { 293-4.2 K Circumferential } \\
\text { Thermal Contraction, } \Delta_{\theta}\end{array}$ & -0.002787 \\
\hline $\begin{array}{r}293-4.2 \mathrm{~K} \mathrm{Axial} \\
\text { Thermal Contraction, } \Delta_{z}\end{array}$ & -0.003295 \\
\hline
\end{tabular}




\section{References for Appendix B.}

1. Urata. M., and Maeda, H. Relation between radial stress and quench current for tightly wound dry solenoids IEEE Trans. MAG-23 (1987) 1596-1599.

2. Bobrov, E.S., Williams, J.E.C., and Iwasa, Y. Experimental and theoret. ical investigation of mechanical disturbances in epoxy-impregnated superconducting coils. 2. Shear-stress-induced epoxy fracture as the principal source of premature quenches and training - theoretical analysis. Cryogenics 25 (1985) 307-316.

3. Williams, J.E.C., and Bobrov, E.S. Magnet system of the $500 \mathrm{MHz}$ spectrometer at the Francis Bitter National Magnet Laboratory: I1. Distrubances, quenches, and training Res. Sci. Instr. 52 (1981) 657-661.

4. Cryogenic Materials Data Handbook AFML-TDR-64-280 Air Force Materials Laboratory, Wright-Patterson Air Force Base, OH (1970).

5. Koch, C.C., and Easton, D.S. A review of mechanical behaviour and stress effects in hard superconductors C'ryogenics 17 (1977) 391-413. 

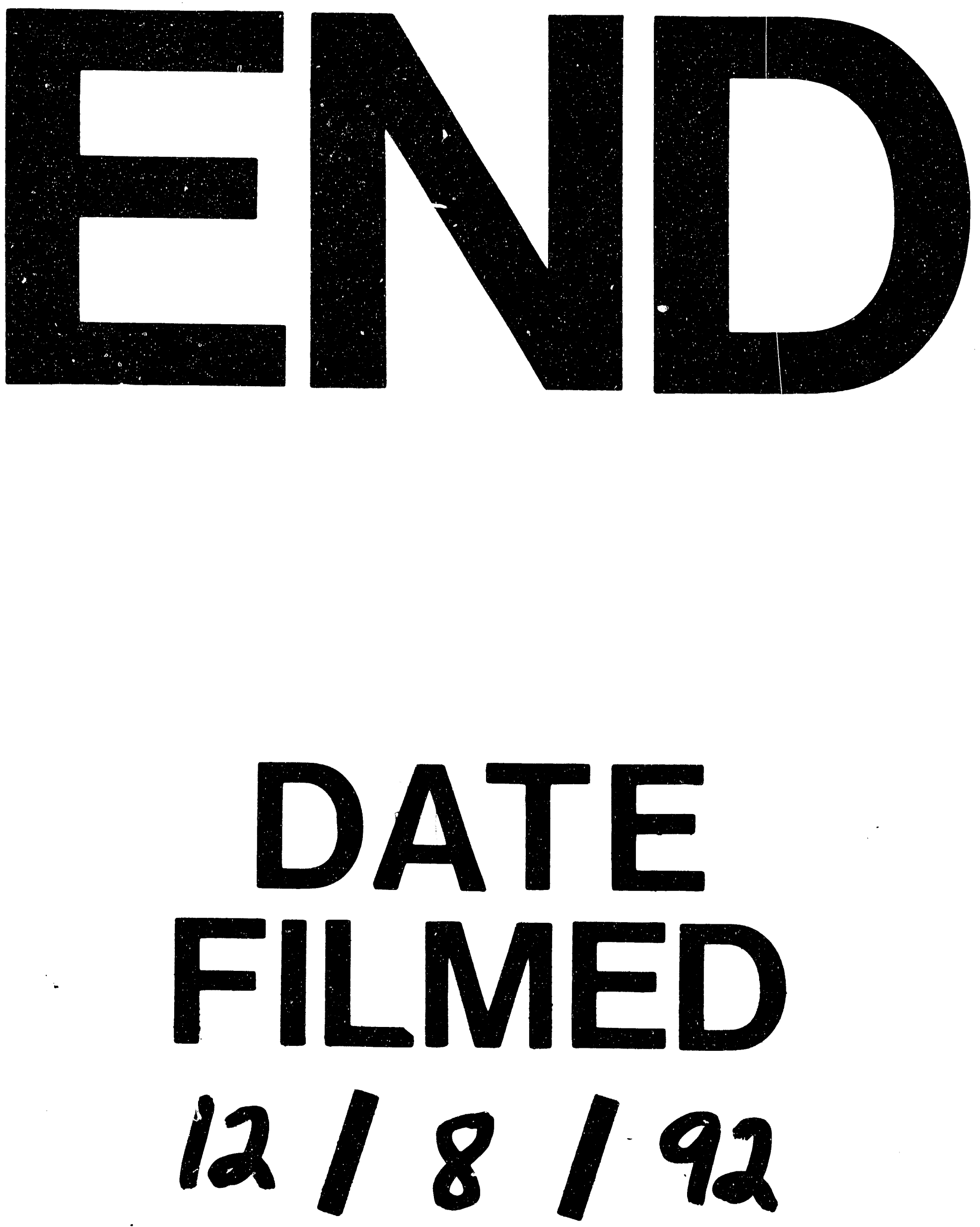
